\title{
MULTICOLOR 3D MINFLUX NANOSCOPY FOR BIOLOGICAL IMAGING
}

\section{DISSERTATION}

for the award of the degree

"Doctor rerum naturalium" (Dr. rer. nat.)

of the Georg-August University Göttingen

within the doctoral program

Physics of Biological and Complex Systems (PBCS)

of the Georg-August University School of Science (GAUSS)

submitted by

\section{Jasmin Kathrin Pape}

from Tuttlingen

Göttingen, 2020 


\section{Thesis Committee}

1. Prof. Dr. Dr. h. c. mult. Stefan W. Hell, Department of NanoBiophotonics, Max Planck Institute for Biophysical Chemistry, Göttingen

2. Prof. Dr. Sarah Köster, Research Group Cellular Biophysics, Institute for X-Ray Physics, Georg-August University, Göttingen

3. Prof. Dr. Claus Ropers, Research Group Nano-Optics and Ultrafast Dynamics, IV. Physical Institute, Georg-August University, Göttingen

\section{Members of the Examination Board}

1. First Reviewer: Prof. Dr. Dr. h. c. mult. Stefan W. Hell, Department of NanoBiophotonics, Max Planck Institute for Biophysical Chemistry, Göttingen

2. Second Reviewer: Prof. Dr. Sarah Köster, Research Group Cellular Biophysics, Institute for X-Ray Physics, Georg-August University, Göttingen

3. Further members of the Examination Board

- Dr. Gopalakrishnan Balasubramanian, Research Group Spin Imaging, Max Planck Institute for Biophysical Chemistry, Göttingen

- Prof. Dr. Stefan Jakobs, Research Group Mitochondrial Structure and Dynamics, Max Planck Institute for Biophysical Chemistry, Göttingen

- Prof. Dr. Silvio Rizzoli, Department of Neuro- and Sensory Physiology, University Medical Center, Göttingen

- Prof. Dr. Claus Ropers, Research Group Nano-Optics and Ultrafast Dynamics, IV. Physical Institute, Georg-August University, Göttingen

\section{Date of the oral examination: 25.02 .2020}


To my beloved family who taught me optimism and hope and never stopped believing in me. 



\section{ABSTRACT}

The resolution of conventional optical fluorescence far-field microscopes is limited by the diffraction of light. This implies that only features in a distance of about half of the wavelength can be discerned. In the last decades, the field of nanoscopy has evolved, theoretically promising molecular resolution by distinguishing close-by fluorescent emitters based on their molecular states that affect the molecules' ability to fluoresce. Due to the limited number of photons that fluorescent molecules can emit before transitioning into a permanent dark state, the resolution of nanoscopy techniques remained limited to about 10-20 nm. The MINFLUX localization approach combines elements of different nanoscopy techniques to achieve true molecular resolution. By probing the position of individually emitting molecules with a targeted minimum of excitation light, the emitted photons are rendered more informative while leaving the photon budget untouched. Compared to a standard camera-based localization scheme, fewer photons are thus required to deduce the position of the molecule with a certain precision. At the inception of this work, MINFLUX delivered an unprecedented localization precision of around $1 \mathrm{~nm}$ when imaging isolated or cellular structures in two dimensions. Estimating the position of the molecule along the optical axis remained to be shown. Moreover, the MINFLUX implementation was limited to the acquisition of a single molecular species, preventing the study of intermolecular distances within biological objects. In this work, I present a MINFLUX nanoscopy approach offering isotropic nanometer precision in three dimensions. This is achieved by probing single molecules with a minimum of excitation light that is confined and targetable in all dimensions. I demonstrate high-fidelity multicolor MINFLUX imaging with molecular resolution in two and three dimensions. I further address the simultaneous tracking of more than one molecular species, which can potentially be applied for studying the dynamics of multi-component objects like protein assemblies. I demonstrate the applicability of 3D multicolor MINFLUX for biological imaging of proteins inside a cellular organelle. Together with an extensive analysis framework, I exploit the 3D isotropic nanometer localization precision as well as the multicolor imaging scheme for quantitatively studying the distribution of different proteins within the heterooligomeric MICOS protein complex in human mitochondria. 



\section{CONTENTS}

1 Introduction 1

1.1 Super-resolution imaging in two and three dimensions . . . . . . . . . 3

1.2 The MINFLUX localization concept . . . . . . . . . . . . . . 6

1.2.1 Experimental implementation . . . . . . . . . . . 7

1.2.2 Calculating a lower bound on the localization precision . . . . 9

1.2.3 Estimating the position of the molecule . . . . . . . . . . . 12

1.2.4 Revisiting localizations in 1D . . . . . . . . . . . 15

1.2.5 Considering background . . . . . . . . . . . . . . . . 19

1.3 Iterative MINFLUX localization . . . . . . . . . . . . . . . . . . 19

1.4 Outline of this thesis . . . . . . . . . . . . . . . . . 22

2 Methods 25

2.1 MINFLUX microscope for 3D and multicolor imaging . . . . . . . . 26

2.1.1 Hardware design . . . . . . . . . . . . . . . . 26

2.1.2 Experiment control software . . . . . . . . . . . . 31

2.1.3 Calibrating the beam position . . . . . . . . . . . . 32

2.1.4 Aberration correction . . . . . . . . . . . . . . . 34

2.2 Daily alignment and data acquisition . . . . . . . . . . . . . . . . . . . . . . . . . . 35

2.3 Data analysis . . . . . . . . . . . . . . . . 37

2.3.1 Segmenting the photon count trace . . . . . . . . . 38

2.3.2 Position estimation . . . . . . . . . . . . . . . 39

2.3.3 Localization filtering . . . . . . . . . . . . . . . . . . . . . . . . . . . . . . . . . 49

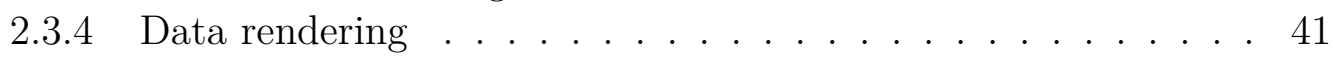

2.3.5 Estimating the localization performance . . . . . . . . . . 42

2.4 Sample preparation . . . . . . . . . . . . . . . . 42

2.4 .1 DNA origami . . . . . . . . . . . . . . . . . . . 42

2.4.2 Fluorescent microspheres . . . . . . . . . . . . . . 44

2.4.3 U-2 OS cells for imaging the nuclear pore complex . . . . . . . 44

2.4.4 Primary hippocampal neurons for PSD-95 imaging . . . . . . 45 
2.4.5 Cell culture and staining for mitochondrial imaging . . . . . . 45

2.4.6 Direct labeling of primary antibodies . . . . . . . . . . . . 46

2.4 .7 Buffers . . . . . . . . . . . . . . . . . 47

2.4.8 Mounting of cell samples . . . . . . . . . . . . 47

3 Imaging in 3D $\quad \mathbf{4 9}$

3.1 Static 3D MINFLUX single emitter localization . . . . . . . . . . 50

3.1.1 Theoretical bounds on the localization precision . . . . . . . 51

3.1.2 Position estimators . . . . . . . . . . . . . . 55

3.2 Iterative MINFLUX in 3D . . . . . . . . . . . . . 56

3.3 MINFLUX imaging in $3 \mathrm{D} \ldots \ldots \ldots \ldots$

3.4 Summary and discussion . . . . . . . . . . . . . . . . . . 61

4 Multicolor localization $\quad \mathbf{6 5}$

4.1 Multicolor MINFLUX for imaging applications . . . . . . . . . . . . 66

4.2 Multicolor MINFLUX for tracking applications . . . . . . . . . . 70

4.2.1 Theory of multicolor MINFLUX tracking . . . . . . . . . . . 71

4.2.2 Experimental implementation . . . . . . . . . . . . 76

4.3 Summary and discussion . . . . . . . . . . . . . . . . . . . . 79

5 Mitochondrial imaging $\quad \mathbf{8 1}$

5.1 Introduction to mitochondrial imaging . . . . . . . . . . . . . . 82

5.2 3D MINFLUX imaging of mitochondrial proteins . . . . . . . . . . 83

5.3 Quantitative molecular analysis of MINFLUX data . . . . . . . . . 85

5.4 3D MINFLUX can resolve protein distributions within the crista junction 93

5.4.1 Mic60 distribution at single crista junctions . . . . . . . . . 93

5.4.2 Distances of subunits within the MICOS complex . . . . . . . 94

5.5 Summary and discussion . . . . . . . . . . . . . . . . . 97

6 Conclusion 101

$\begin{array}{llr}7 & \text { Bibliography } & 109\end{array}$

$\begin{array}{ll}\text { A Appendix figures } & 121\end{array}$

$\begin{array}{ll}\text { B DNA strands } & 127\end{array}$ 


\section{Abbreviations}

1D

one-dimensional

2D

two-dimensional

$3 \mathrm{D}$

three-dimensional

AOTF

acousto-optic tunable filter

ADP

adenosine diphosphate

ATP

adenosine triphosphate

BSA bovine serum albumin

CRB Cramér Rao bound

DNA deoxyribonucleic acid

DMEM Dulbecco's modified Eagle's medium

DMF dimethylformamid

DTT dithiothreitol

EGFP enhanced green fluorescent protein

FOV field of view

FPGA field-programmable gate array

FRET fluorescence resonance energy transfer

FWHM full-width at half-maximum

ID identifier

IgG immunoglobulin G

KO knockout

MEA cysteamine hydrochloride

MICOS mitochondrial contact site and cristae organizing system

mLMSE modified least mean square estimator

MLE maximum likelihood estimator

NHS 1-Hydroxy-2,5-pyrrolidinedione

NPC nuclear pore complex

numLMSE numerically unbiased least mean square estimator

PC personal computer

PCA principal component analysis

PDF probability density function 
PID proportional-integral-derivative

PBS phosphate-buffered saline

PEG polyethylene glycol

PSF point spread function

RESOLFT reversible saturable optical fluorescence transitions

RGB red-green-blue color space

SLM spatial light modulator

SBR signal-to-background ratio

STED stimulated emission depletion

STORM stochastic optical reconstruction microscopy

TAE Tris-acetate-EDTA

TCP targeted coordinate pattern

TIR total internal reflection

USB universal serial bus

UV ultraviolet

VFL varifocal lens

WGA wheat germ agglutinin 


\section{INTRODUCTION}

Biological structures of interest to medicine and biological research span several orders of magnitude in size, ranging from whole multi-cellular organisms ( $1 \mathrm{~mm}-10 \mathrm{~m})$ and organelles $(\sim 1 \mu \mathrm{m})$ down to single proteins $(\sim 10 \mathrm{~nm})$ or atoms $(\sim 1 \AA)$. Aiming for the understanding of biological mechanisms and functions on different scales, a multitude of imaging modalities has been invented over the last centuries. The oldest form of biological imaging is optical imaging. It makes use of the interaction of the biological object with visible light. In its native form, optical imaging happens in the human sensory system when we perceive our environment with the naked eye. Early on, the detail that could be observed with visible light was enhanced by the manufacturing of lenses. In the 16th century, a combination of lenses was used for the first time to observe biological structures, marking the invention of the optical microscope. These early microscopes already revealed new details of life, including the first observation of a cell by Robert Hooke in 1665. Over the centuries, the resolution of optical microscopes, meaning the size of the smallest structure that could be observed, was improved by the optimization of the employed optical components. In 1873, Ernst Abbe postulated that these optimizations could never lead to unlimited resolution because even when using perfect optical elements, the observed image would still be blurred by the diffraction of light [1]. This implied that only structures about $200 \mathrm{~nm}$ apart should be discernible with visible light. The postulated limit remained valid for more than a century, also after the invention of fluorescence microscopy that tremendously improved the specificity of the imaging by tagging the structure of interest with a probe. The fluorescent probe absorbs light and subsequently emits light of a longer wavelength, so that only the structure of interest is observed, while its surrounding appears dark. In 1994, Stefan Hell proposed to exploit the properties of fluorescent dyes to break the diffraction limit, marking the advent of super-resolution microscopy or nanoscopy [51]. A first experimental demonstration exploiting stimulated emission to control the electronic state of the fluorescent molecules, followed only a few years later [63]. At about the same time, US scientists developed approaches to detect the fluorescence from single molecules [76], later leading to another class of nanoscopy approaches [10, 93]. 
With the resolving power of nanoscopy reaching down to few tens of nanometers in experiments, important discoveries were made including the periodicities in the cytosceletal network of neurons [18, 122], the detailed measurement of changes in neuronal morphologies inside living tissue $[109,111]$ or even in the brain of a living mouse [9] as well as the observation of the ring-like structure of Bax assemblies in apoptosis $[37,96]$.

As a consequence, the Nobel foundation awarded the Nobel Prize in Chemistry 2014 to Eric Betzig, Stefan W. Hell and William E. Moerner for the development of super-resolved fluorescence microscopy.

In theory, super-resolution microscopy techniques provide molecular resolution. In experiments, the finite number of photons that a fluorescent molecule can emit before it transitions to a permanent dark state, limits the resolution to few tens of nanometers. MINFLUX, introduced in 2016, achieves true molecular resolution by fundamentally improving the fluorescence photon usage of the molecular observation while keeping the photon budget unaltered [6]. Single fluorescent molecules are illuminated with an excitation beam of known shape. The beam features an intensity minimum that is targeted to positions as close to the molecule as possible. The target positions define an adapted coordinate system for the localization. By additionally exploiting the knowledge of the beam shape, the illumination thus provides supplementary information for the localization. This implies that, in comparison to standard camera-based approaches, fewer fluorescence photons are required for precise knowledge of the molecule's location. So far, MINFLUX facilitated the localization of single molecules with 22-fold improved photon efficiency compared to a standard camera-based localization. Moreover, imaging with single nanometer precision and tracking of single molecules in living cells with unprecedented spatiotemporal resolution was demonstrated $[6,26]$. Up to recently, the technique only allowed a precision improvement in $2 \mathrm{D}$ without providing axial information. Further, it only allowed imaging of small objects, because the field of view (FOV) was confined to about $100 \mathrm{~nm}$ in diameter.

Recently, iterative MINFLUX facilitated the acquisition of cellular structures with nanometer precision in a micrometer-sized field of view $[42,43]$. In this thesis, I set forth multicolor and 3D MINFLUX imaging. The results have been published in Nature Methods alongside iterative MINFLUX [42]. I further study the applicability of the technique for biological imaging of cellular organelles. The results have been published in [81] after the oral examination of this thesis.

In the present chapter, I provide the reader with an overview of super-resolution microscopy approaches for 2D and 3D imaging and elaborate on the theoretical background and experimental realization of static and iterative MINFLUX localization. 


\subsection{Super-resolution imaging in two and three dimensions}

When relying solely on the focusing of light, the resolution of an optical microscope, meaning its ability to discern close-by features, is limited to

$$
d_{x y}=0.51 \frac{\lambda}{n \sin \theta}=0.51 \frac{\lambda}{\mathrm{NA}},
$$

where the resolution $d_{x y}$ is defined to be the full-width at half-maximum (FWHM) of the point spread function (PSF) of a microscope with a numerical aperture NA. The numerical aperture is defined as the product of the refractive index $n$ of the immersion medium and the sine of the half-opening angle of the objective lens $\theta$. Several advances were made to push the resolution limit, including confocal microscopy $[82,121]$, structured illumination microscopy [40, 46, 71], 4Pi microscopy [52] or two-photon microscopy [20], all of them leaving the physical boundaries imposed by diffraction in place.

In contrast, optical super-resolution microscopy techniques break the resolution limits imposed by the wave-nature of light by introducing a new concept to distinguish close-by emitters, namely the preparation of fluorescent molecules in distinct electronic states [49]. By exploiting these molecular states, super-resolution microscopy techniques can deliver molecular resolution in theory.

Stimulated emission depletion (STED) microscopy was the first super-resolution technique truly breaking the diffraction limit by distinguishing close-by fluorescent emitters through control of their electronic states [49]. The technique was theoretically proposed in 1994 [51] and experimentally demonstrated a few years later [63]. It relies on the targeted suppression of fluorescence in the rim of a focused excitation beam. This is implemented based on stimulated emission through illumination with a donut-shaped STED beam. Photons from spontaneous emission are distinguished by wavelength-separation. The optical resolution of a STED microscope $d_{\text {STED }}$ increases with the intensity of the off-switching STED light and is given by

$$
d_{\mathrm{STED}} \approx \frac{d_{\mathrm{conf}}}{\sqrt{1+I_{\mathrm{STED}} / I_{\mathrm{S}}}},
$$

where $d_{\text {conf }}$ is the resolution of the underlying confocal microscope, $I_{\mathrm{STED}}$ is the intensity at the donut crest and $I_{\mathrm{s}}$ is the saturation intensity, at which the fraction of spontaneously emitted photons drops to $1 / 2$ [49]. The saturation intensity depends on the wavelength and pulse length of the STED beam as well as on the photo-physical properties of the employed fluorescent dye [44].

Super-resolution microscopy techniques that use the spatially targeted switching of molecules, as for example STED microscopy, will here-after be called coordinate- 
targeted super-resolution microscopy techniques. A widefield-based approach to super-resolution, later termed coordinate-stochastic, was presented few years after the first implementation of stimulated emission depletion (STED) [10, 93, 100]. The approach uses the stochastic on-/off-switching of fluorescent emitters in time, so that within a diffraction-limited region on the camera, only one emitter is allowed to fluoresce at a time. The emitter's position can be estimated with much higher precision $\sigma_{\mathrm{loc}, x y}$ than the optical resolution of the microscope would suggest, so that a high-resolution image can be reconstructed from a series of sparse camera images. The attainable localization precision for a Gaussian emission PSF with width $\sigma_{\text {fluo, } x y}$ is given by

$$
\sigma_{\text {loc }, x y}=\frac{\sigma_{\text {fluo }, x y}}{\sqrt{N}},
$$

when disregarding experimental imperfections $[11,47,78] . N$ describes the number of photons detected for a given localization. When using an unbiased position estimator [21], the localization precision is directly proportional to the resolution of the microscope

$$
d_{\mathrm{CAM}}=2 \sqrt{2 \ln (2)} \cdot \sigma_{\mathrm{loc}},
$$

defining resolution again as the FWHM of the expected image of a point source.

In coordinate-stochastic as well as in coordinate-targeted super-resolution techniques imaging emitters with nanometer isotropic resolution also along the direction of the optical axis remains challenging. Two main classes of approaches were implemented by (i) encoding axial information through modification of the point-spread-function in the illumination (coordinate-targeted, [63]) or fluorescence (coordinate-stochastic [62, 102]) or (ii) by using the interference of light focused (coordinate-targeted, $[12,50$, 97] or detected (coordinate-stochastic, $[4,103,112]$ ) by two opposing objective lenses. Stochastic 3D super-resolution microscopy techniques are well-summarized in [21, 117] and are of ongoing interest regarding improved localization precisions as well as an extended axial imaging range $[55,59,101]$. Recently, a theoretical study presented the measurement-independent quantum Cramér Rao bound (CRB) on the precision that can be achieved with any standard camera-based 3D localization approach and studied how well the quantum CRB is reached by the proposed techniques [5]. The authors derived that a two-objective approach combined with an interferometric detection can indeed reach the quantum-theoretical bound with values

$$
\begin{aligned}
\sigma_{x y} & \sim 70 \mathrm{~nm} / \sqrt{N} \\
\sigma_{z} & \sim 50 \mathrm{~nm} / \sqrt{N},
\end{aligned}
$$

for a numerical aperture of NA $=1.4$ and a wavelength $\lambda=670 \mathrm{~nm}$ when using $N$ photons for a single localization. Equation 1.6 readily shows that even in an ideal experimental situation, the photon budget of the fluorescent molecules, meaning how many photons the molecule emits before bleaching, can limit the attainable resolution. 
Coordinate-targeted approaches do not require many fluorescence photons to achieve high resolution because targeting the illumination beam defines the position of the molecule that is allowed to emit. High switching intensities increase the photobleaching rates of the illuminated molecules, however, so that molecular resolution has not been achieved in biological imaging. Several approaches have been presented that reduce the bleaching rate of the molecules. Besides the direct modification of fluorescent dyes, these include buffer optimization [114], moving to cryogenic temperatures $[119,120]$ or repeatedly exchanging the fluorescent molecule that targets a structure of interest [61, 100].

MINFLUX, first presented in 2016, improved the photon efficiency of the localization process compared to standard camera-based approaches rather then engineering the photon budget. The technique thus requires an order of magnitude fewer fluorescence photons for a certain localization precision [6]. The concept combines elements of both coordinate-targeted and coordinate-stochastic approaches. Like in coordinatestochastic approaches, the emission state of the molecules is controlled, so that only one single molecule is allowed to emit within a diffraction-limited volume. By targeting a minimum of excitation light, e.g. a donut-shaped beam, several times as precisely as possible to the emitting molecule, the beam positions define a reduced coordinate system in the nanometer-vicinity of the fluorophore. The information that the fluorescence photons have to carry is thus substantially reduced compared to a conventional camera-based localization scheme.

Within a limited FOV, MINFLUX allowed an improvement of roughly 20-fold in photon efficiency. In imaging experiments, a localization precision of about $1 \mathrm{~nm}$ was measured. The resolution power was demonstrated by imaging molecules only $6 \mathrm{~nm}$ apart. In tracking experiments in living bacterial cells, MINFLUX delivered 100-fold more localizations per trace compared to conventional camera-based single molecule tracking approaches. A localization precision of $2 \mathrm{~nm}$ with a temporal resolution of $400 \mu \mathrm{s}$ was demonstrated later [26].

Following the introduction of the MINFLUX concept, combinations of structured illumination with stochastic blinking of single molecules were proposed to overcome the limitations of the first MINFLUX implementation with respect to the reduced field of view $[16,38,89]$. These techniques offer a mild improvement in localization precision compared to standard camera-based approaches, but allow the simultaneous localization of single molecules in a micrometer-sized FOV. With the introduction of iterative MINFLUX (see Section 1.3), image regions of several micrometers size in (living) cells are also accessible with MINFLUX, reaching much higher photon efficiencies than any camera-based approach [43]. An extension of MINFLUX for localizing with nanometer isotropic precision in 3D, a multicolor implementation and first biological applications were developed within this work and are presented in Chapters 3-5. In the next section, the MINFLUX concept and the underlying theory are introduced in more detail (Section 1.2), followed by an explanation of the iterative MINFLUX approach (Section 1.3). 


\subsection{The MINFLUX localization concept}

In MINFLUX, single emitting molecules are illuminated with a targeted minimum of excitation light using a beam of known intensity distribution. The position of the molecule is obtained exploiting both the unlimited photon number of the illuminating light and the limited number of fluorescence photons. The MINFLUX principle is readily explained in the one-dimensional (1D) case using excitation beams with a quadratic shape $I_{i}=c_{\mathrm{b}} x^{2}$ with $c_{\mathrm{b}}$ denoting the constant intensity scaling of the beam. If the intensity zero of the illumination beam coincides with the position of the fluorescent molecule, meaning $x_{\mathrm{m}}=0$, no fluorescence photons are collected, but the molecule's position can still be estimated. Assuming the molecule to be displaced with respect to the intensity minimum, so that $x_{\mathrm{m}} \neq 0$, the number of collected fluorescence photons scales with the illumination intensity leading to a mean Poissonian emission of $\lambda_{i}=b_{\mathrm{m}} \cdot I_{i}\left(x_{\mathrm{m}}\right)=b_{\mathrm{m}} \cdot c_{\mathrm{b}} \cdot x_{\mathrm{m}}^{2}$ with $b_{\mathrm{m}}$ being the effective molecular brightness depending on the detection efficiency of the microscope and the photo-physical properties of the fluorescent molecule.

When illuminating the molecule with a single beam and assuming $b_{\mathrm{m}}$ to be known with arbitrary precision, the distance between the molecule and the zero position can be estimated from the photon number $n$. Using the estimator $\hat{\lambda}=n$ with standard deviation $\Delta \hat{\lambda}=\sqrt{n}$, the estimate of the distance between molecule and the zero-position of the excitation beam is given by

$$
\left|\hat{x}_{\mathrm{m}}\right|=\sqrt{\frac{\hat{\lambda}}{c_{\mathrm{b}} b_{\mathrm{m}}}} .
$$

Using a Gaussian error propagation approach we obtain a localization error of

$$
\begin{aligned}
\Delta\left|\hat{x}_{\mathrm{m}}\right| & =\frac{\partial\left|\hat{x}_{\mathrm{m}}\right|}{\partial \hat{\lambda}} \cdot \Delta \hat{\lambda} \\
& =\frac{1}{2 \sqrt{b_{\mathrm{m}} c_{\mathrm{b}}}} \frac{1}{\sqrt{\hat{\lambda}}} \cdot \Delta \hat{\lambda} \\
& =\frac{1}{2 \sqrt{b_{\mathrm{m}} c_{\mathrm{b}}}} .
\end{aligned}
$$

The error of the distance estimate thus only scales with the gradient of the illumination function and does not depend on the position of the molecule nor the number of emitted photons. With decreasing distance between molecule and excitation minimum, fewer photons are emitted for the same illumination conditions, relating to an increased photon efficiency. If the position of the molecule coincides with the excitation minimum, the molecule does not emit any photons. Still, the localization error does not diverge, but remains constant.

Due to the symmetry of the excitation beam, two exposures are necessary to estimate 
the absolute position of the molecule. Assume we illuminate the molecule in two subsequent exposures with their zero positions at $x_{0}=-L / 2$ and $x_{1}=+L / 2$ in a distance $L$. We collect two photon numbers $n_{0}$ and $n_{1}$. As before, the mean of each photon number is proportional to the illumination intensity at the position of the molecule $\lambda_{i}=b_{\mathrm{m}} \cdot I_{i}\left(x_{\mathrm{m}}\right)$. The estimator for the mean of each of the Poisson distributions is again given by $\hat{\lambda}_{i}=n_{i}$ with an error of $\Delta \hat{\lambda}_{i}=\sqrt{n_{i}}$. The molecule's position is readily estimated by combining

$$
\begin{aligned}
& \lambda_{0}=b_{\mathrm{m}} c_{\mathrm{b}}\left(x_{\mathrm{m}}-x_{0}\right)^{2} \\
& \lambda_{1}=b_{\mathrm{m}} c_{\mathrm{b}}\left(x_{\mathrm{m}}-x_{1}\right)^{2}
\end{aligned}
$$

to obtain

$$
\frac{\hat{\lambda}_{0}}{\left(\hat{x}_{\mathrm{m}}-x_{0}\right)^{2}}-\frac{\hat{\lambda}_{1}}{\left(\hat{x}_{\mathrm{m}}-x_{1}\right)^{2}}=0 .
$$

By solving the quadratic equation we find the estimator of the molecule position

$$
\begin{aligned}
\hat{x}_{\mathrm{m}} & =\frac{\sqrt{n_{0}}-\sqrt{n_{1}}}{\sqrt{n_{0}}+\sqrt{n_{1}}} \frac{L}{2} \\
& =\frac{L}{1+\sqrt{n_{1} / n_{0}}}-\frac{L}{2},
\end{aligned}
$$

which we observe to be independent of the molecular brightness and beam power. This is an important outcome, as the molecular brightness for a single molecule is usually hard to assess and can vary strongly depending on the experimental situation. With a rigorous approach to the prediction of an achievable localization precision (Section 1.2.2) and the general derivation of position estimators (Section 1.2.3), we will reconsider the presented 1D case in Section 1.2.4.

To convince the reader of the experimental applicability of MINFLUX, we here continue with a description of the first experimental implementation and discuss published MINFLUX acquisition modalities.

\subsubsection{Experimental implementation}

In the last section, we showed how using a targeted minimum of excitation light can improve the photon efficiency of the localization. This section is dedicated to a brief explanation of the experimental implementation of MINFLUX [6, 26] and summarize the results that were achieved for different experimental modalities of MINFLUX. Both studies used a 2D donut-shaped beam, as known from STED microscopy, for targetable excitation (Figure 1.1). The excitation beam sequentially pointed to each of four multiplex positions. The positions were arranged in a triangle with additional 
central exposure (Figure 1.2). The arrangement of positions used for MINFLUX multiplexing is called targeted coordinate pattern (TCP) throughout this work. The combinations of photons collected in the individual exposures within a multiplex cycle were then used to infer the position of the molecule.

The employed microscope was essentially a confocal microscope as schematically displayed in Figure 1.1. The excitation beam was amplitude-modulated to adjust the emission rate of the single molecule under observation and to switch the beam off while repositioning. A vortex phase mask modulated the wavefront of the beam, so that a donut-shaped intensity distribution with a central minimum was formed at the focal plane of the objective lens. Electro-optical deflectors repositioned the beam for multiplexing. The same objective lens collected the fluorescence light that was then spatially filtered using a confocal pinhole and detected on a single-photon counting device. A beam of ultraviolet (UV) light was co-aligned with the excitation beam and focused into the sample to photo-activate or photo-convert individual molecules for imaging or tracking experiments.

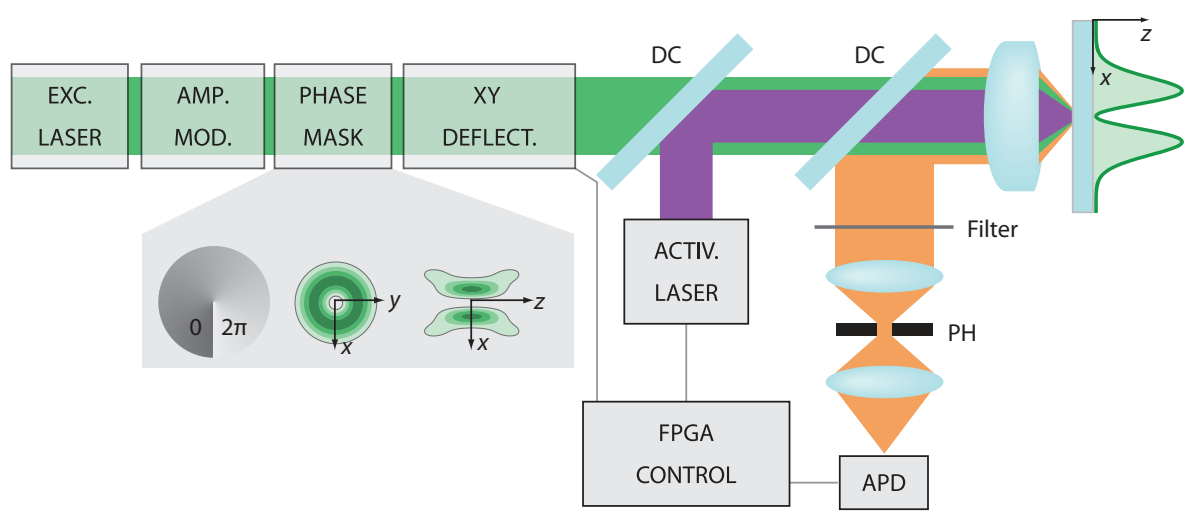

Figure 1.1: Basic 2D MINFLUX system. An excitation beam (green) is amplitude and phase-modulated (inlet, vortex phase mask and resulting intensity distribution), deflected in $x y$ for MINFLUX multiplexing and focused into the sample, where it forms a donut-shaped pattern. The activation beam (violet) is co-aligned with the excitation beam using a dichroic mirror, so that a Gaussian-shaped activation spot is formed in the sample. Fluorescence (orange) is collected in a confocal detection. A field-programmable gate array (FPGA) board controls the scanners and reads out the detector. Adapted from [43].

We can differentiate between two MINFLUX modalities: single molecule localization microscopy (nanoscopy) and single molecule tracking. When using MINFLUX for nanoscopy (Figure 1.2a), molecules are forced to emit sequentially in time, for example by using the cyanine dye Alexa Fluor 647 in combination with a redox buffer system as known from STORM microscopy [113]. The molecule positions are thus individually probed and estimated, so that an image can be assembled from all estimated molecule positions. Using DNA origami samples, MINFLUX achieved $\sim 1 \mathrm{~nm}$ localization precision in 2D using only 500 photons and resolved molecules 
that were only $6 \mathrm{~nm}$ apart, a regime previously inaccessible to super-resolution microscopy using conventional probes [6]. The improvement in photon efficiency was limited to the region within the targeted coordinate pattern (TCP), however, so that the position of the origami had to be pre-assigned based on a widefield camera image. An approach for the extension of the field of view for cellular MINFLUX imaging with nanometer resolution [43] is presented in Section 1.3.

When using MINFLUX for single molecule tracking (Figure 1.2b), either continuously emitting spatially isolated organic dye molecules [26] or photo-convertible fluorescent proteins in living cells [6] were localized. To follow the movement of molecules over regions larger than the TCP, the position of the molecule was continuously estimated on an FPGA board. Based on the estimated position, the TCP center was re-adjusted, to continue the MINFLUX acquisition of the molecule in the photon-efficient region [6]. In tracking experiments over extended regions, MINFLUX reached an up to 100-fold improvement compared to conventional camera-based single-molecule tracking implementations, so that 100 times more localizations were acquired before the molecule bleached [6]. An even higher temporal resolution can
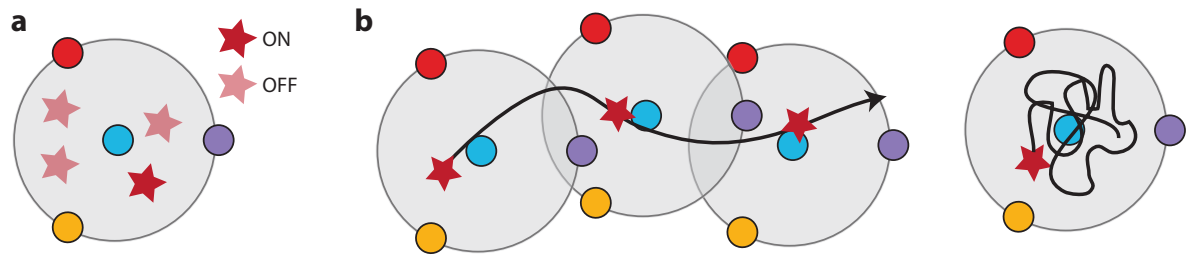

Figure 1.2: Experimental modalities of MINFLUX. a, MINFLUX imaging using an on-/off transition to acquire single molecules (red stars) sequentially in time [6]. Colored dots represent the TCP. b, MINFLUX tracking to follow single, continously emitting molecules (red stars) over extended regions (left) [6] or within the confined MINFLUX FOV (right) [26].

be achieved when probing small movements within a TCP-sized region [26], so that no repositioning of the TCP is required (Figure 1.2b, right). This approach allowed for localizations with $\sim 2 \mathrm{~nm}$ precision within time intervals of only $400 \mu \mathrm{s}$ [26]. It is important to note that by changing the photon collection time for an individual localization, the MINFLUX tracking experiment can be tuned between high temporal and high spatial resolution.

Based on the improved photon efficiency, MINFLUX entered a new spatio-temporal regime of single molecule experiments that is further extend within this work (see Section 1.4).

\subsubsection{Calculating a lower bound on the localization precision}

All MINFLUX modalities benefit from the possibility to tune the photon efficiency of the localization by changing the size of the TCP and the negligible influence of the wavelength on the localization precision. Based on an information-theoretical 
approach, these important properties of MINFLUX localization are deduced in detail in [6], [25] and [41]. Here, we only briefly outline how this information-theoretical approach based on a statistical treatment of the photon collection can be used to predict a lower bound on the localization precision for any MINFLUX experiment.

Assume we illuminate a molecule located at a position $\vec{r}_{\mathrm{m}}$ with a series of $K$ excitation beams with spatially varying intensity $I_{i}(\vec{r})=I\left(\vec{r}-\vec{r}_{i}^{(\mathrm{b})}\right)$, displaced to a position $\vec{r}_{i}^{(\mathrm{b})}$. At each beam position, we collect a number of photons $n_{i}$ following a Poisson distribution

$$
p\left(n_{i}\right)=\frac{\lambda_{i}^{n_{i}}}{n_{i} !} \cdot \mathrm{e}^{-\lambda_{i}}
$$

where $\lambda_{i}\left(\vec{r}_{\mathrm{m}}\right)=c_{\mathrm{e}} \cdot \beta_{\mathrm{m}} \cdot I_{i}\left(\vec{r}_{\mathrm{m}}\right)=b_{\mathrm{m}} \cdot I_{i}\left(\vec{r}_{\mathrm{m}}\right)$ is a function of the molecule's position $\vec{r}_{\mathrm{m}}$. In the following we do not explicitly write the dependence in order to improve legibility. The apparent brightness $b_{\mathrm{m}}$ of the molecule is determined by the detection efficiency $c_{\mathrm{e}}$ of the imaging system, and the intrinsic brightness $\beta_{\mathrm{m}}$ of the molecule. The intrinsic molecular brightness depends on the quantum yield and the absorption cross section at the excitation wavelength. Without loss of generality, we assume to collect a total number of $N$ photons. The conditional probability for collecting a set of photons $\left\{n_{i}\right\}$, given that $\sum_{i=0}^{K-1} n_{i}=N$, follows a multinomial distribution

$$
P\left(\left\{n_{i}\right\} \mid N\right)=\frac{N !}{n_{0} ! \cdots n_{K-1} !} \prod_{i=0}^{K-1} p_{i}^{n_{i}}
$$

with the entries of the multinomial parameter vector being

$$
p_{i}\left(\vec{r}_{\mathrm{m}}\right)=\frac{\lambda_{i}}{\sum_{j=0}^{K-1} \lambda_{j}} \approx \frac{I_{i}\left(\vec{r}_{\mathrm{m}}\right)}{\sum_{j=0}^{K-1} I_{j}\left(\vec{r}_{\mathrm{m}}\right)}
$$

for $i \in[0, \ldots, K-1]$. The approximation looses validity if there is more than one molecule emitting at a given time (see Section 4.2). For a detailed derivation of the multinomial distribution from the Poisson distribution the reader is referred to [41]. We derive a lower bound on the localization precision by calculating the Fisher information that the photon numbers hold on the position of the molecule. We assume a probability density function (PDF) $P(\vec{X} \mid \vec{\theta})$, where $\vec{X}$ is a set of random variables, here the photon numbers $\left\{n_{i}\right\}$, and $\vec{\theta}$ a parameter vector of the PDF, here the parameter vector of the multinomial distribution $\vec{p}$. The information that the realization $\vec{X}$ carries on the parameters $\vec{\theta}$ depends on the average sharpness of the peak in the likelihood function $\mathcal{L}(\vec{\theta} \mid \vec{X})$. In more mathematical terms, the Fisher information matrix is defined as

$$
\mathcal{I}_{i j}(\vec{\theta})=\mathrm{E}_{\vec{X}}\left[\frac{\partial^{2}}{\partial \theta_{i} \partial \theta_{j}} \ln \mathcal{L}(\vec{\theta} \mid \vec{X})\right]
$$


with $\mathrm{E}_{\vec{X}}$ denoting the expected value with respect to the set of random variables $\vec{X}$. In the case of a MINFLUX localization, we can calculate the Fisher information $\mathcal{I}(\vec{p})$ that the photon numbers $\left\{n_{i}\right\}$ hold on the multinomial parameter vector $\vec{p}$. Based on a re-parametrization, the Fisher information on the molecule position $\mathcal{I}\left(\vec{r}_{\mathrm{m}}\right)$ is then given by

$$
\mathcal{I}\left(\vec{r}_{\mathrm{m}}\right)=\mathcal{J}^{T} \mathcal{I}(\vec{p}) \mathcal{J}
$$

with $\mathcal{J}$ being the Jacobian matrix with entries

$$
\mathcal{J}_{i j}=\frac{\partial p_{i}}{\partial x_{j}}
$$

describing the coordinate transformation from the spatial coordinate space $\vec{r}_{\mathrm{m}}$ to the reduced $\vec{p}$-space. The space of multinomial parameters is reduced because it contains only $K-1$ independent parameters for $K$ exposures, as $\sum_{i=0}^{K-1} p_{i}=1$ holds. This means that the indices $i$ and $j$ are restricted to $i \in\{0, \ldots, K-2\}$ and $j \in\{1, \ldots, \Delta\}$, respectively, where $K$ is the number of exposures, and $\Delta$ is the number of spatial variables to be estimated. The number of spatial variables is determined by the dimensionality of the localization and the number of molecules to be localized.

Further, we know that the likelihood for $\vec{p}$, given that we measure $\left\{n_{i}\right\}$, equals the conditional probability function

$$
\mathcal{L}\left(\vec{p} \mid\left\{n_{i}\right\}\right)=P\left(\left\{n_{i}\right\} \mid N\right) .
$$

Using the above definitions, the Fisher information on the parameter vector $\vec{p}$ is

$$
\mathcal{I}_{i j}(\vec{p})=N\left(\frac{1}{p_{K-1}}+\delta_{i j} \frac{1}{p_{i}}\right)
$$

leading to the Fisher information on the position of the molecule

$$
\mathcal{I}\left(\vec{r}_{\mathrm{m}}\right)=N \sum_{i=0}^{K-1} \frac{1}{p_{i}}\left[\begin{array}{ccc}
\left(\frac{\partial p_{i}}{\partial x_{1}}\right)^{2} & \cdots & \frac{\partial p_{i}}{\partial x_{1}} \frac{\partial p_{i}}{\partial x_{\Delta}} \\
\vdots & \ddots & \vdots \\
\frac{\partial p_{i}}{\partial x_{\Delta}} \frac{\partial p_{i}}{\partial x_{1}} & \cdots & \left(\frac{\partial p_{i}}{\partial x_{\Delta}}\right)^{2}
\end{array}\right]
$$

As stated in the Cramér Rao inequality $[17,88]$, the lower limit on the covariance matrix $\boldsymbol{\Sigma}$ of any unbiased estimator is given by the inverse of the Fisher information matrix

$$
\boldsymbol{\Sigma}\left(\vec{r}_{\mathrm{m}}\right) \geq \mathcal{I}^{-1}\left(\vec{r}_{\mathrm{m}}\right)=: \boldsymbol{\Sigma}^{(\mathrm{CRB})}\left(\vec{r}_{\mathrm{m}}\right),
$$

so that the inverse of the Fisher information matrix defines the CRB. Throughout 
this work, we used the one-dimensional standard deviations

$$
\sigma_{i}=\sqrt{\Sigma_{i i}^{(\mathrm{CRB})}\left(\vec{r}_{\mathrm{m}}\right)}
$$

and the average $1 \mathrm{D}$ error

$$
\sigma_{1 D}=\sqrt{\frac{1}{\Delta} \operatorname{tr}\left(\Sigma^{(\mathrm{CRB})}\right)}
$$

to characterize the MINFLUX localization precision. We further define the isotropy

$$
\alpha=\sqrt{\frac{\min _{i \in\{1, \ldots, D\}} \lambda_{i}^{(\mathrm{CRB})}}{\max _{i \in\{1, \ldots, D\}} \lambda_{i}^{(\mathrm{CRB})}}}
$$

of the localization, where $\lambda_{i}^{(\mathrm{CRB})}$ is the $i$-th eigenvalue of the CRB matrix $\boldsymbol{\Sigma}^{(\mathrm{CRB})}$.

\subsubsection{Estimating the position of the molecule}

The CRB formulated in Section 1.2.2 defines a lower bound on the localization precision when using an unbiased estimator. Three types of estimators have been presented for MINFLUX localization approaches [6]. A maximum likelihood estimator (MLE) was used for asymptotically unbiased position estimation in post-processing. A numerically simple modified least mean square estimator (mLMSE) allowed for live position estimation on an FPGA board. Due to the strong bias of the MLE in the low-photon regime, a numerically unbiased least mean square estimator (numLMSE) was developed for unbiased position estimation in post-processing, if localizing based on few photons. In this work, only MLE and mLMSE were used and are introduced in this section.

\section{Maximum likelihood estimator}

The MLE maximizes the likelihood $\mathcal{L}\left(\vec{r}_{\mathrm{m}} \mid\left\{n_{i}\right\}\right)$ that a molecule is located at a position $\vec{r}_{\mathrm{m}}$ given a measured set of photon numbers $\left\{n_{i}\right\}$. By definition, maximum-likelihood estimators are (1) consistent, meaning that they converge to the true parameter value $\vec{r}_{\mathrm{m}}$ for $N \rightarrow \infty$, (2) asymptotically Gaussian as the estimates approach a Gaussian distribution for $N \rightarrow \infty$ and (3) asymptotically efficient, meaning that the covariance of the estimated parameters reaches the CRB for $N \rightarrow \infty$. Another important property of the MLE is its invariance

$$
g\left(\hat{\theta}_{\mathrm{MLE}}\right)=[g \hat{(\theta)}]_{\mathrm{MLE}}
$$

with $g(\theta)$ being a function $\mathbb{R}^{n} \rightarrow \mathbb{R}^{m}$.

To evaluate the estimator performance for a MINFLUX localization under given 
conditions, the localization is repeated for $M$ sets of randomly generated photon counts. The multinomial photon counts are generated based on the parameter vector $\vec{p}$ (see Equation 1.19) assuming a molecule position $\mu_{x_{i}}$. For each set of photon numbers $m$, the position $\hat{x}_{i, m}$ is estimated. The average estimated position in the $i$ th dimension is denoted by $\bar{x}_{i}$. The $1 \mathrm{D}$ standard deviation of the localization is determined by

$$
\sigma_{i}=\sqrt{\frac{1}{M} \sum_{m=1}^{M}\left(\bar{x}_{i}-\hat{x}_{i, m}\right)^{2}} .
$$

A comparison of the covariance matrix of the estimated positions to the CRB indicates whether estimator efficiency has been reached. The average 1D bias of the position estimation is defined as

$$
\beta_{i}=\bar{x}_{i}-\mu_{x_{i}}
$$

and provides information on the consistency of the estimator. Often, the aim is to minimize the total localization error given by

$$
\mathcal{E}_{i}=\sqrt{\sigma_{i}^{2}+\beta_{i}^{2}}
$$

The localization properties formulated in Equations 1.31, 1.32 and 1.33 are used to quantify estimator performances throughout this work. For some geometries, an analytic expression for the maximum-likelihood estimation of the molecule position can be found (see e.g. Section 1.2.4 for analytic expression of estimators in 1D). In this work, a numerical implementation of the MLE was used in post-processing or for the numerical evaluation of the MINFLUX performance. Details of the numerical implementation are described in Section 2.3.2.

\section{Modified least mean square estimators}

To obtain a numerically simple estimator for the molecule position, assuming that the molecule is positioned close to the TCP center, we can use the linear approximation of the multinomial parameter vector $\vec{p}(\vec{r})$ (Equation 1.19) around the origin $\vec{r}=0$ using a Taylor series expansion

$$
p_{i}(\vec{r})=p_{i}(\vec{r}=0)+\left.\sum_{j=1}^{D} r_{j} \frac{\partial p_{i}}{\partial r_{j}}\right|_{\vec{r}=0}+\mathcal{O}\left(\vec{r}^{2}\right) .
$$

The approximation of the multinomial parameter vector is then given by

$$
\vec{p}(\vec{r}) \approx \vec{p}(\vec{r}=0)+\mathcal{J}_{\mathbf{0}} \cdot \vec{r}
$$

with $\mathcal{J}_{\mathbf{0}}$ being the Jacobian matrix of the coordinate transformation from $\vec{r}$-space to reduced $\vec{p}$-space evaluated at the TCP center. Using the invariance property of 
the MLE (Equation 1.30) we can write

$$
\hat{\vec{p}}-\vec{p}(\vec{r}=0)-\mathcal{J}_{\mathbf{0}} \cdot \hat{\vec{r}}_{\mathrm{m}}=0
$$

Equation 1.36 is an overdetermined system of linear equations that can be solved using a least mean square approach

$$
\hat{\vec{r}}_{\mathrm{LMS}}=\underset{\vec{r}_{\mathrm{m}}}{\operatorname{argmin}}\left\|\hat{\vec{p}}-\vec{p}(\vec{r}=0)-\mathcal{J}_{\mathbf{0}} \cdot \hat{\vec{r}}_{\mathrm{m}}\right\|^{2}
$$

that has the solution

$$
\hat{\vec{r}}_{\mathrm{LMS}}=\left(\mathcal{J}_{\mathbf{0}}^{\top} \mathcal{J}_{\mathbf{0}}\right)^{-1} \mathcal{J}_{\mathbf{0}}^{\top}(\hat{\vec{p}}-\vec{p}(\vec{r}=0)) .
$$

For a detailed derivation of Equation 1.38 the reader is referred to [104]. We should note here, that Equation 1.38 assumes $\mathcal{J}_{\mathbf{0}}$ to have full rank for the inverse of $\mathcal{J}_{\mathbf{0}}^{\top} \mathcal{J}_{\mathbf{0}}$ to exist.

We can derive the mLMSE for the 4-point 2D-donut excitation pattern displayed in Figure 1.2. We assume exposures with intensity distribution

$$
I(\vec{r})=A_{0} 4 \mathrm{e} \ln (2) \frac{\left(\vec{r}-\vec{r}_{i}^{(\mathrm{b})}\right)^{2}}{\mathrm{FWHM}^{2}} \mathrm{e}^{-4 \ln (2) \frac{\left(\vec{r}-\vec{r}_{i}^{(\mathrm{b})}\right)^{2}}{\mathrm{FWHM}^{2}}},
$$

where $\vec{r}_{\mathrm{b}_{i}}$ describes the position of the intensity minimum in the $i$ th exposure and FWHM the full width at half-maximum of the beam. In the described case, Equation 1.38 takes the form

$$
\hat{\vec{r}}_{\mathrm{LMS}}=-\frac{1}{1-\frac{L^{2} \ln (2)}{\mathrm{FWHM}^{2}}} \sum_{i=1}^{3} \hat{p}_{i} \cdot \vec{r}_{i}^{(\mathrm{b})},
$$

where the parameter $L$ describes the size of the TCP. Unsurprisingly, the estimator does not show any dependence on the multinomial success probability of the central exposure as the first derivative of $p_{0}$ vanishes. To also consider the counts in the central exposure, we write an mLMSE

$$
\hat{\vec{r}}_{\mathrm{mLMS}}=\frac{1}{1-\frac{L^{2} \ln (2)}{\mathrm{FWHM}^{2}}}\left(\sum_{j=0}^{k} \beta_{j} \hat{p}_{0}^{j}\right)\left(\sum_{i=1}^{3} \hat{p}_{i} \cdot \vec{r}_{i}^{(\mathrm{b})}\right),
$$

with constant estimator parameters $\beta_{j}$. The modification introduces an increased radial scaling of the estimated position with growing $\hat{p}_{0}$, so that the parameters $\beta_{j}$ have to be numerically optimized for a given signal-to-background ratio (SBR) and beam separation $L[6]$. Due to its numerically simple form, we used the mLMSE with estimator orders $j \in\{0,1\}$ for live position estimation similarly to previous 
works (see Section 2.2) [6, 26, 43].

\subsubsection{Revisiting localizations in 1D}

As an illustrative example, we consider bounds and estimators for a 1D localization with two exposures. We first write the general CRB and MLE for a 1D localization and then compare the results for quadratic and Gaussian excitation beam shapes to motivate the use of an intensity minimum for localization. For the localization performance when using other beam shapes the reader is referred to [6].

Assume a molecule is fixed at a 1D position $x_{\mathrm{m}}$ and subsequently excited by displaced intensity distributions of the form $I_{0}(x)=I(x-L / 2)$ and $I_{1}(x)=I(x+L / 2)$, so that we detect photon numbers $n_{0}$ and $n_{1}$ adding up to $N=n_{0}+n_{1}$. Following Equation 1.19 the multinomial success probability is given by

$$
p_{i}(x)=\frac{I_{i}(x)}{I_{0}(x)+I_{1}(x)} .
$$

Lower bound on the localization precision Based on Equation 1.24, we determine the Fisher information

$$
\mathcal{I}(\vec{p})=N \cdot\left(\frac{1}{1-p_{0}}+\frac{1}{p_{0}}\right)
$$

that the counts $n_{0}$ and $n_{1}$ hold on the multinomial parameters. Using the Jacobian of the transformation from coordinate space $x_{\mathrm{m}}$ to reduced $\vec{p}$-space (Equation 1.22)

$$
\mathcal{J}=\frac{\partial p_{0}}{\partial x}
$$

together with Equation 1.21, we obtain the Fisher information

$$
\mathcal{I}(x)=N \cdot \frac{1}{p_{0}\left(1-p_{0}\right)} \cdot\left(\frac{\partial p_{0}}{\partial x}\right)^{2}
$$

that the photon counts hold on the position of the molecule. In the $1 \mathrm{D}$ case, the CRB is simply the lower bound on the variance for any unbiased position estimation, so that the minimal standard deviation of localizations obtained from an unbiased estimator is given by

$$
\begin{aligned}
\sigma_{x} & =\frac{1}{\sqrt{\mathcal{I}(x)}} \\
& =\frac{1}{\sqrt{N}} \frac{\sqrt{p_{0} \cdot\left(1-p_{0}\right)}}{\left|\partial p_{0} / \partial x\right|}
\end{aligned}
$$


Maximum likelihood estimator We first consider the estimator of the multinomial parameter vector and use the likelihood function as defined by Equations 1.23 and 1.18 , so that we obtain

$$
\mathcal{L}\left(\vec{p} \mid\left\{n_{i}\right\}\right)=\frac{N !}{n_{0} ! n_{1} !} \prod_{i=0}^{1} p_{i}^{n_{i}}
$$

for the $1 \mathrm{D}$ case with two exposures. As the logarithm is a monotonic function, we can reduce computational complexity by determining the maximum of the logarithm of the likelihood function instead of the maximum of the likelihood function directly. We write

$$
\begin{aligned}
\left.\frac{\mathrm{d} \ln \mathcal{L}\left(\vec{p} \mid\left\{n_{i}\right\}\right)}{\mathrm{d} p_{0}}\right|_{\hat{p}_{0, \mathrm{MLE}}} & =\left.\frac{\mathrm{d}\left[\ln \left(\frac{N !}{n_{0} ! n_{1} !}\right)+n_{0} \ln p_{0}+n_{1} \ln \left(1-p_{0}\right)\right]}{\mathrm{d} p_{0}}\right|_{\hat{p}_{0, \mathrm{MLE}}} \\
& =n_{0} \frac{1}{p_{0}}-\left.n_{1} \frac{1}{1-p_{0}}\right|_{\hat{p}_{0, \mathrm{MLE}}} \\
& \stackrel{!}{=} 0
\end{aligned}
$$

where $\hat{p}_{0, \mathrm{MLE}}$ is the maximum likelihood estimate of parameter $p_{0}$ that is consequently given by

$$
\hat{p}_{0, \mathrm{MLE}}=\frac{n_{0}}{n_{0}+n_{1}}
$$

Similarly, we can calculate the maximum likelihood estimate of the molecule position by using the likelihood function

$$
\mathcal{L}\left(x \mid\left\{n_{i}\right\}\right)=\frac{N !}{n_{0} ! n_{1} !} \prod_{i=0}^{1}\left[\frac{I_{i}(x)}{I_{0}(x)+I_{1}(x)}\right]^{n_{i}}
$$

and again calculating the maximum of the log-likelihood function

$$
\left.\frac{\mathrm{d} \ln \mathcal{L}\left(x \mid\left\{n_{i}\right\}\right)}{\mathrm{d} x}\right|_{\hat{x}_{\mathrm{MLE}}} \stackrel{!}{=} 0 .
$$




\section{Quadratic beams}

Like in Section 1.2, we define the intensity distributions as

$$
\begin{aligned}
& I_{0}(x)=c_{\mathrm{b}}\left(x+\frac{L}{2}\right)^{2} \\
& I_{1}(x)=c_{\mathrm{b}}\left(x-\frac{L}{2}\right)^{2},
\end{aligned}
$$

where the scaling factor $c_{\mathrm{b}}$, influenced by changing the exposure time or by adjusting the overall beam power, determines the curvature of the beam. Using Equation 1.42, the spatial dependence of the multinomial parameters is described by

$$
p_{0,1}(x)=\frac{1}{2} \frac{\left(x \pm \frac{L}{2}\right)^{2}}{x^{2}+\frac{L^{2}}{4}} .
$$

Based on Equation 1.47, we obtain the lower bound on the localization precision

$$
\sigma_{x, \mathrm{CRB}}=\frac{1}{\sqrt{N}} \frac{L}{4}\left(1+\left(\frac{x}{L / 2}\right)^{2}\right),
$$

notably scaling with the beam separation $L$, meaning that the photon efficiency can be easily improved by reducing the distance between the illuminating beams. Further, the lower bound is independent of the curvature of the illumination. Remarkably, the best localization precision can be achieved in the center $x=0$ between the two exposures. The localization error increases towards the beam positions $x=$ $\pm L / 2$, but stays within $L /(4 \sqrt{N}) \leq\left[\sigma_{x, \mathrm{CRB}}(|x|<L / 2)\right] \leq L /(2 \sqrt{N})$. Based on Equation 1.54, the maximum likelihood position estimator has two solutions. Only one satisfies $|x|<L / 2$, so that the position estimator can be written as

$$
\hat{x}_{\mathrm{MLE}}=\frac{L}{1+\sqrt{n_{1} / n_{0}}}-\frac{L}{2},
$$

delivering the result already derived in Section 1.2.

\section{Gaussian beams}

Analogously we consider two exposures with Gaussian-shaped beams with intensity scaling constant $c_{\mathrm{b}}$ and width FWHM positioned in a distance $L$

$$
I_{0}(x)=c_{\mathrm{b}} \cdot \mathrm{e}^{-4 \ln (2) \frac{(x+L / 2)^{2}}{\mathrm{FWHM}^{2}}}
$$




$$
I_{1}(x)=c_{\mathrm{b}} \cdot \mathrm{e}^{-4 \ln (2) \frac{(x-L / 2)^{2}}{\mathrm{FWHM}^{2}}} .
$$

The lower bound on the localization precision

$$
\sigma_{x, \mathrm{CRB}}=\frac{1}{4 \ln (2)} \frac{\mathrm{FWHM}^{2}}{L \sqrt{N}} \cosh \left(x \frac{4 L \ln (2) \cdot}{\mathrm{FWHM}^{2}}\right)
$$

is obtained based on Equation 1.47. Using the first-order series expansion cosh $(a x)=$ $1+\mathcal{O}\left(x^{2}\right)$ at $x=0$, we immediately see an inverse scaling of the precision with the beam separation $L$. Notably the error shows a quadratic dependence on the beam width FWHM for molecules close to the TCP center. This implies that the presented localization scheme only delivers a better precision than conventional camera-based approaches if $L>$ FWHM holds. The MLE

$$
\hat{x}_{\mathrm{MLE}}=\frac{\mathrm{FWHM}^{2}}{8 \ln (2) L} \ln \left(\frac{n_{0}}{n_{1}}\right)
$$

is again derived from Equation 1.54 as demonstrated in more detail in [6]. Due to its simple form, the MLE for Gaussian beams in 1D is also used for live position estimation. The position estimation is readily expanded to $2 \mathrm{D}$ when using a cross-like TCP, for which the localizations in $x$ and $y$ are linearly independent.

When comparing the lower bounds on the localization precision for excitation with Gaussian and parabolic beams, we see that in both cases, the precision can be tuned with the beam separation $L$. This is an important result on its own, as it implies that we can modify the localization precision with a geometric parameter that we can adapt easily, especially compared to the photon number $N$ or the size of the diffraction-limited beam. In a first order approximation, the precision for Gaussian illuminations follows an inverse dependence on the beam separation $L$. This implies a reduced localization error for large beam separations and molecules close to the TCP origin. The opposite is true for parabolic excitation beams, where shrinking the beam separation reduces the error on the position estimation. This makes the use of an excitation minimum favorable for localization, as aberrations usually influence the rim of the excitation beams, making the intensity distribution for $x>$ FWHM hard to control experimentally. Also, with growing distance to the beam center, the approximation of the regularly focused excitation beam with a Gaussian function looses validity, whereas a donut beam is well approximated with a parabola in the photon-efficient regime assuming $L \ll$ FWHM. For covering larger areas, the weak dependence of the localization precision on the position of the molecule within the TCP can make the use of Gaussian beams favorable (see Section 1.3). For photonefficient localization steps, we exploited a targetable minimum of excitation light throughout this work. 


\subsubsection{Considering background}

Under experimental conditions, background emission due to auto-fluorescence from the coverslip, buffer or sample or due to fluorescence emission from other molecules cannot be avoided. The theory presented until here, however, did not take background emission into account. Here, we introduce a background treatment as previously described [6]. When assuming the background to obey a Poisson distribution with mean $\lambda_{i}^{(\mathrm{bg})}$ and the emission of the molecule to take a mean $\lambda_{i}^{(\mathrm{m})}$, the multinomial parameter for each exposure (see Equation 1.19) takes the form

$$
p_{i}\left(\vec{r}_{\mathrm{m}}\right)=\frac{\lambda_{i}^{(\mathrm{m})}\left(\vec{r}_{m}\right)+\lambda_{i}^{(\mathrm{bg})}\left(\vec{r}_{m}\right)}{\sum_{j=0}^{K-1}\left\{\lambda_{j}^{(\mathrm{m})}\left(\vec{r}_{m}\right)+\lambda_{j}^{(\mathrm{bg})}\left(\vec{r}_{m}\right)\right\}} .
$$

We can define the SBR [6]

$$
\begin{aligned}
\operatorname{SBR}\left(\vec{r}_{\mathrm{m}}\right) & =\frac{\sum_{i=0}^{K-1} \lambda_{i}}{\sum_{i=0}^{K-1} \lambda_{i}^{(\mathrm{bg})}} \\
& =\frac{b_{\mathrm{m}} \sum_{i=0}^{K-1} I_{i}\left(\vec{r}_{\mathrm{m}}\right)}{\sum_{i=0}^{K-1} \lambda_{i}^{(\mathrm{bg})}\left(\vec{r}_{\mathrm{m}}\right)} .
\end{aligned}
$$

Under the assumption of equal background contributions in all exposures, we can combine Equations 1.64 and 1.66 and obtain

$$
p_{i}\left(\vec{r}_{\mathrm{m}}, \operatorname{SBR}\right)=\frac{\operatorname{SBR}\left(\vec{r}_{\mathrm{m}}\right)}{\operatorname{SBR}\left(\vec{r}_{\mathrm{m}}\right)+1} \cdot p_{i}^{(0)}\left(\vec{r}_{\mathrm{m}}\right)+\frac{1}{\operatorname{SBR}\left(\vec{r}_{\mathrm{m}}\right)+1} \cdot \frac{1}{K}
$$

with $p_{i}^{(0)}$ being the background-free $p$-function as defined in Equation 1.19.

The signal level in a MINFLUX acquisition depends on (i) the brightness of the molecule $b_{\mathrm{m}}$, (ii) the position of the molecule $\vec{r}_{\mathrm{m}}$ with respect to the beam positions and (iii) with respect to the confocal detection as well as (iv) on the beam separation $L$. An important conclusion is, that there is an optimal value $L_{\mathrm{opt}}$ for each background level as lowering the beam separation $L$ decreases the SBR. If choosing $L$ too small, background contributions will dominate and impede a photon-efficient localization [26]. The dependencies of the signal level also imply the that SBR can strongly vary between localizations, so that it has to be determined individually (see Section 2.3.2).

\subsection{Iterative MINFLUX localization}

The drastic improvement in photon efficiency in the static MINFLUX implementation is restricted to emitters that are located within the TCP region of size $L$. The improvement additionally shows a dependence on the exact emitter position as 
a

b $-n_{0}-n_{1}-n_{2}-n_{3}$
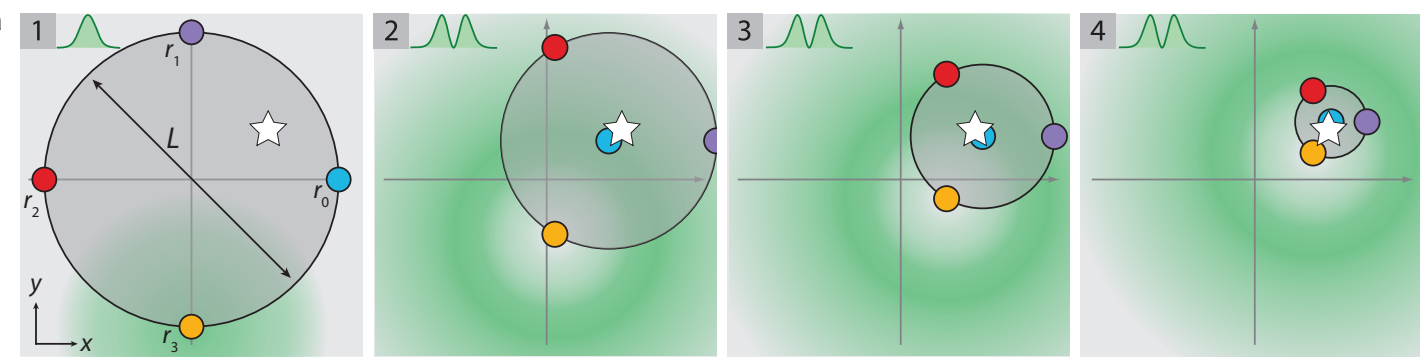

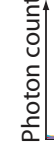

c

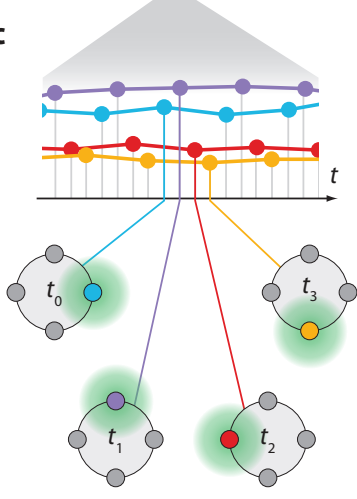

d
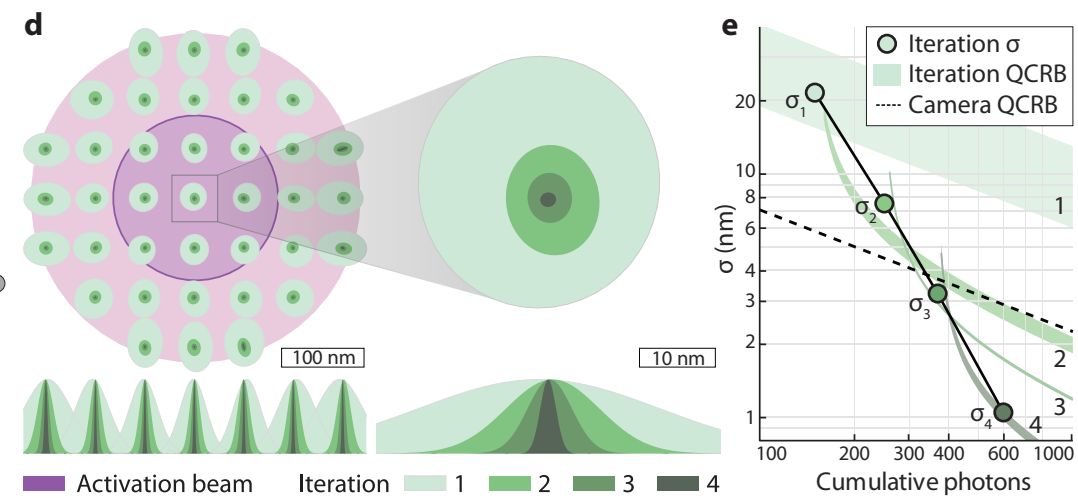

Figure 1.3: Iterative MINFLUX concept in two dimensions. a, Predefined TCP steps with beam separation $L$ and discrete beam positions (colored dots) were used to approach the molecule (white star) by performing a live estimation of the position. Different beam shapes were used in the iterations, as depicted in form of a green sketch and overlaid with the last exposure (yellow dot). b, Photon count traces for the different iterations. The count rate of the central exposure (blue) is reduced upon centering the TCP onto the molecule. c, The MINFLUX TCP is applied in discrete steps by switching off the excitation beam and moving to the next position in between exposures. The photon count numbers are combined to form count traces as displayed in (b). d, Simulated localization precision for iteration steps as depicted in (a). Photon numbers $N_{i}=\{150,100,120,230\}$ delivering a total photon number of $N_{\text {tot }}=600$ and beam separations $L_{i}=\{300 \mathrm{~nm}, 150 \mathrm{~nm}, 90 \mathrm{~nm}, 40 \mathrm{~nm}\}$ were used. In the first iteration, Gaussian beams were used for excitation. In iterations 2-4 donut-shaped beams excited the molecule. The covariances of the position estimation (green shades) are shown as ellipses of $\mathrm{e}^{-1 / 2}$ level. The activation area determines the region that has to be covered with the iterative scheme (purple: FWHM $=200 \mathrm{~nm}$ with $50 \%$ activation probability, pink: $2 \cdot$ FWHM with $95 \%$ activation probability). e, Spatial average of precisions $\sigma_{1 \cdots 4}$ shown in (d) (colored dots), together with the CRB in the iterations (green shades). The quantum CRB for a conventional camera-based localization is shown (dashed line). Material from: Gwosch, K.C.*, Pape, J.K.*, Balzarotti, F.* et al., MINFLUX nanoscopy delivers 3D multicolor nanometer resolution in cells, Nature Methods, 2020, Springer Nature. 
illustrated in Section 1.2.4 and experimentally studied for the 2D case in [6]. This trade-off between photon efficiency and FOV impedes the photon-efficient imaging of larger regions. This is especially true for non-isolated objects in cells, where the diffraction-limited activation beam can photo-activate molecules outside the TCP.

Iterative MINFLUX overcomes the limitations imposed by the trade-off between information content and range of action [43]. This imaging procedure offers high photon efficiency in arbitrarily large regions and facilitates imaging in fixed and living cells. This is accomplished by iteratively approaching each photo-activated emitter with a set of TCP arrangements, while gradually shrinking the TCP size $L$. This allows to (i) reach any fluorophore within a photo-activated region (typically $200 \mathrm{~nm}$ FWHM), (ii) have an isotropic localization precision in 2D and (iii) surpass the typical $\propto 1 / \sqrt{N}$ dependence, as photons are made increasingly informative as they are acquired. The concept of iterative MINFLUX was first theoretically described in [41] and experimentally demonstrated in [43].

The power of iterative MINFLUX is readily explained in 1D. Consider a molecule at $x_{\mathrm{m}}=0$ that is excited with two subsequent quadratic beams at positions $x_{0}=-L_{0} / 2$ and $x_{1}=L_{0} / 2$. After acquiring a total number of $N_{0}$ photons, the CRB on the position estimation (Equation 1.58) is given by

$$
\sigma_{0}\left(x_{\mathrm{m}}=0\right)=\frac{1}{\sqrt{N_{0}}} \frac{L_{0}}{4} .
$$

After collecting $N_{0}$ photons and localizing the molecule, we can shrink the distance between the excitation beams to $L_{1}=3 \sigma_{0}$, so that the new localization precision scales with

$$
\begin{aligned}
\sigma_{1}\left(x_{\mathrm{m}}=0\right) & =\frac{1}{\sqrt{N_{1}}} \frac{L_{1}}{4} \\
& =\frac{1}{\sqrt{N_{1}}} \frac{3 \sigma_{0}}{4} \\
& =\frac{1}{\sqrt{N_{0} N_{1}}} \frac{3 L_{0}}{4^{2}} .
\end{aligned}
$$

Repeating the same process $k$ times leads to

$$
\sigma_{k}\left(x_{\mathrm{m}}=0\right)=\frac{1}{\sqrt{N_{0} N_{1} \cdots N_{k}}} \frac{3^{k}}{4^{k+1}} L_{0} .
$$

Choosing the photon number to be equal in all iterations and using an overall photon number $N_{\text {tot }}$, so that $\frac{N_{\text {tot }}}{k+1}=N_{0}=\cdots=N_{k}$ holds, we obtain

$$
\sigma_{k}\left(x_{\mathrm{m}}=0\right)=\left(\frac{k+1}{N}\right)^{\frac{k+1}{2}} \frac{3^{k}}{4^{k+1}} L_{0},
$$


demonstrating that centering and shrinking the excitation beam distance onto the molecule can outperform the waiting for more photons. In this way, iterative MINFLUX breaks the $\sigma \propto \frac{1}{\sqrt{N}}$ dependence that limits conventional camera-based localization techniques and static MINFLUX approaches.

\section{Experimental implementation of iterative MINFLUX in 2D for scanning large image regions}

In a first experimental implementation, 2D iterative MINFLUX used a number of discrete zooming steps as depicted in Figure 1.3a. Beam shapes, beam separations $L_{i}$ and photon numbers $N_{i}$ were predefined for each iteration. After applying the discrete positions of the TCP in iteration $i$ (Figure 1.3c) and acquiring the photon trace (Figure 1.3b) until collecting $N_{i}$ photons, the position of the molecule was estimated using the live position estimator for the employed TCP. The center position of the TCP was re-adjusted and the settings for the TCP of the next iteration applied as depicted. As described in Section 1.2.5, the choice of the final beam separation was adjusted to an optimum for the present SBR. Figure 1.3c shows an ellipse representation of the simulated localization precision in each iteration for different molecule positions within the activation region. The final localization precision is largely isotropic and independent of the position of the molecule, which is in stark contrast to static MINFLUX implementations [6]. When inspecting the spatially averaged localization precision in each iteration (Figure 1.3d), it becomes apparent that using iterative MINFLUX, the localization error indeed does not scale with $1 / \sqrt{N}$. For the given implementation, the error approximately followed a $1 / N^{2}$ behavior. The photon efficiency of conventional camera-based localization was surpassed at roughly $N_{\text {tot }} \sim 400$ photons and reached a $1 \mathrm{D}$ precision of $1 \mathrm{~nm}$ when using a total photon number of about $N_{\text {tot }} \sim 600$. By scanning the iterative FOV together with the activation beam over the sample, large image regions were acquired with a localization precision of about $1 \mathrm{~nm}$ [43]. Also proof-of concept measurements in living cells were performed demonstrating the compatibility of MINFLUX imaging with living cells [43].

\subsection{Outline of this thesis}

Iterative MINFLUX, as presented so far, allows imaging of cellular samples with nanometer localization precision in both lateral dimensions. It does not allow, however, to extract any information about the axial position of the fluorophores. Biological processes inherently happen in three dimensions, so that knowing the position of the fluorophores in the axial direction as well is essential especially to draw quantitative conclusions.

Further, fluorescence labeling offers very high specificity at the cost of rejecting 
any information about the environment of the fluorescent molecules. To interpret fluorescence images, it is thus often required to image at least two target structures simultaneously.

The aim of this work was the development and implementation of a multicolor 3D MINFLUX nanoscopy approach that allows drawing quantitative conclusions from imaging data acquired with nanometer isotropic precision. To this end, I first developed a three-dimensional MINFLUX scheme for imaging with isotropic nanometer localization precision in a micrometer-sized FOV (Chapter 3). I established a multicolor imaging approach to distinguish two or more fluorescent species in a single specimen, preserving nanometer precision for both species (Chapter 4). With the aim of recording the time evolution of molecular distances, I theoretically studied the MINFLUX localization of two simultaneously emitting fluorophores with distinct spectral properties. I performed first proof-of-concept measurements with two immobile, but simultaneously emitting fluorophores (Chapter 4). I optimized and applied a protocol for first 3D multicolor MINFLUX imaging of structures inside an organelle. More specifically, the experiments allowed to study the protein distribution within the heterooligomeric MICOS protein complex that is located at crista junctions in human mitochondria. I worked out an analysis pipeline to extract distance information and to isolate molecular arrangements of single crista junctions. Based on these tools, I studied the distances and distributions of components within the MICOS complex with unprecedented precision (Chapter 5).

All methods and materials used in this work are introduced in Chapter 2.

Parts of this thesis have been published in [42] with pre-release in [43], so that figures as well as written text are partially adapted from this work. Further, the data shown in Chapter 5 in this thesis have been published in [81] after first submission of this thesis. 



\section{METHODS}

In the previous chapter I laid out the theoretical grounds of MINFLUX required for the understanding of this thesis and formulated the goals of the presented work. The present chapter is dedicated to the introduction of experimental methods and materials that are required to meet these goals. This includes aspects of optics design, electronics, software development and data or image processing as well as protocols for the preparation of reference and biological samples.

I first describe the hard- and software design of the MINFLUX microscope (Sections 2.1.1 and 2.1.2). Further, I elaborate on calibration procedures specifically developed for 3D MINFLUX localization, including an approach for the measurement of the axial scaling introduced by a refractive index mismatch between the coverslip and the sample medium (Section 2.1.3) and a pupil-segmentation-based measurement scheme for the correction of system aberrations (Section 2.1.4). I further comment on daily alignment procedures and MINFLUX data acquisition schemes (Section 2.2). I elaborate on the basic MINFLUX data analysis (Section 2.3), including a trace segmentation for the identification of molecular emission events and a maximum likelihood estimation for localization. The reaction to background or emission events outside the MINFLUX imaging region require a reliable event filtering approach that I describe in detail. I define data rendering strategies and present an approach for the quantification of the experimental localization precision. I conclude the chapter with protocols for the preparation of all buffers and technical as well as biological samples used within this work (Section 2.4). 


\subsection{MINFLUX microscope for 3D and multicolor imaging}

The microscope used and expanded within this work was based on the hardware design already described in $[6,26]$ as well as the software introduced in [43]. In brief: the hardware was constituted of a confocal fluorescence microscope with ability for fast beam position and power modulation as well as arbitrary phase modulation of the excitation beam wavefront allowing for the generation of an excitation minimum (Section 2.1.1). We addressed the main hardware components using an FPGA board that additionally enabled real-time position estimation based on the detected photons and subsequent re-adjustment of the TCP (Section 2.1.2). We describe the beam position calibration along the optical axis (Section 2.1.3) as well as an aberration correction approach that we used reduce beam distortions to a minimum (Section 2.1.4).

\subsubsection{Hardware design}

This section is dedicated to the in-detail description of the microscope hardware that was used in this work. The minimal functional components of a MINFLUX system suitable for 2D acquisitions have already been introduced in Section 1.2.1. In brief, MINFLUX requires the generation of an intensity minimum that can be targeted to different positions in the sample. Fluorescence photons emitted by the labeling molecules in the specimen have to be collected, so far all MINFLUX implementation have used a confocal detector for this task. A photo-activation beam switched single fluorescent molecules for individual registration.

The full design of the optical microscope used in this work is schematically displayed in Figure 2.1 and Table 2.1 contains a list of all specialized hardware components. The design of the microscope can be subdivided in different segments that are indicated in Figure 2.1 and described below. Concisely, we used a widefield illumination and camera detection to identify a region of interest in the sample. The region of interest was addressed using either the sample stage or a large-range piezo-scanner. During MINFLUX acquisition, the sample was illuminated with different point illumination beams, including the photo-activation beam and two types of targeted excitation beams. Fluorescence was detected on confocal detectors that additionally allowed for multicolor classification. To avoid drift of the sample during MINFLUX measurements, a stabilization system actively controlled the sample position. The software and hardware interfaces that were used for the microscope control are described in Section 2.1.2.

Point illumination For MINFLUX acquisition, we selected between three point illumination beam paths. For excitation, we either used a Gaussian or a donut- 


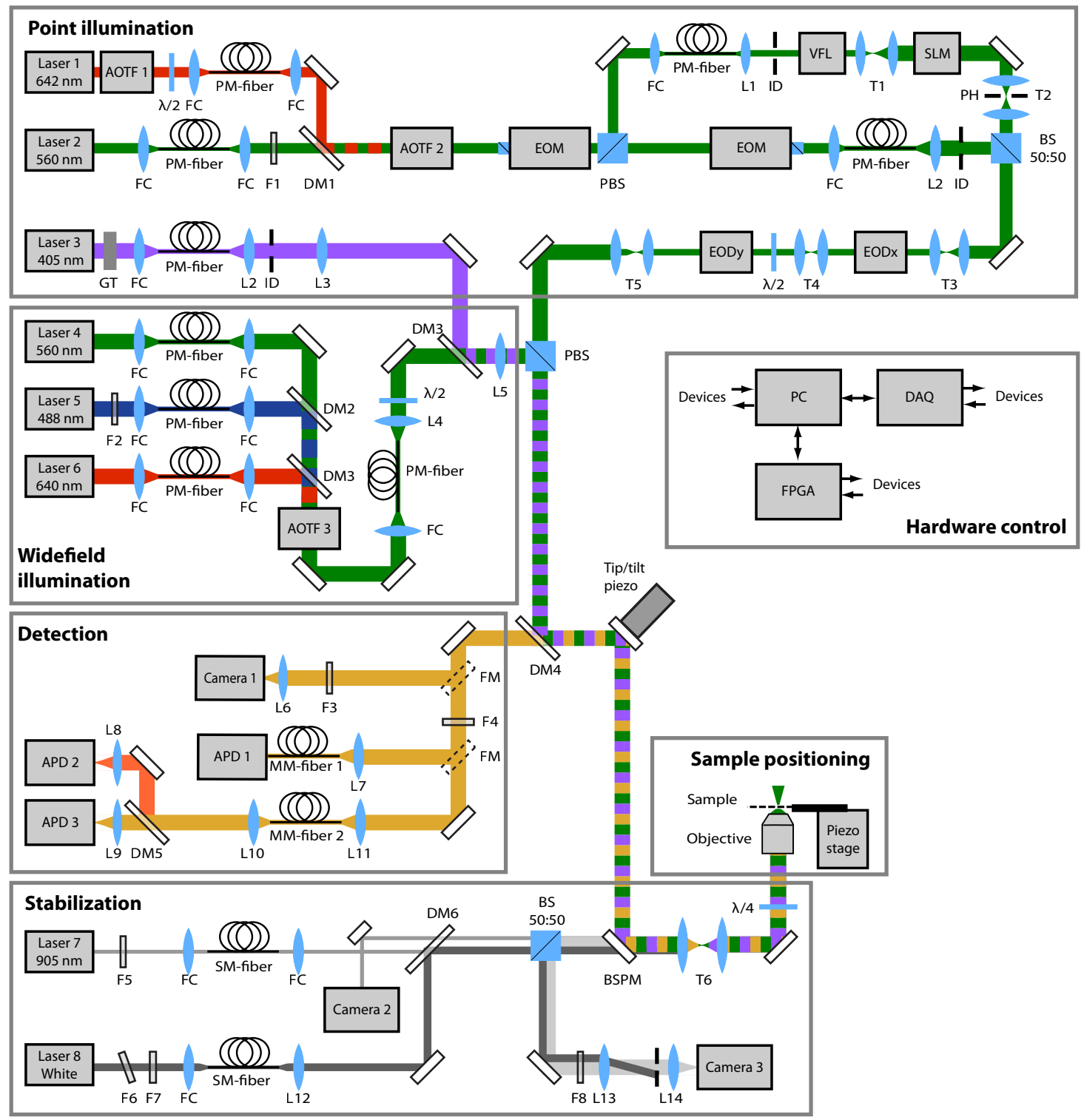

Figure 2.1: MINFLUX microscope for 3D multicolor imaging. We used a point illumination in combination with a confocal detection for MINFLUX acquisition. To choose a region of interest in the sample, we used a widefield illumination and a single-molecule sensitive camera. A stabilization system ensured nanometer stability during MINFLUX acquisition. An FPGA and other data acquisition boards controlled the hardware components. Table 2.1 contains a list of all components shown here. Material from: Gwosch, K.C.*, Pape, J.K.*, Balzarotti, F.* et al., MINFLUX nanoscopy delivers 3D multicolor nanometer resolution in cells, Nature Methods, 2020, Springer Nature. 
shaped beam (560 nm or $642 \mathrm{~nm}$ ), for photo-activating single fluorescent molecules we illuminated the sample with UV light of $405 \mathrm{~nm}$ wavelength. An acousto-optic tunable filter (AOTF) allowed to adapt the beam power and wavelength. We selected one of the two excitation beam types using a combination of two electro-optic modulators (EOMs). We mode-cleaned all illumination beams using single-mode polarization-maintaining fibers. A varifocal lens (VFL) quickly defocused the donutshaped beam to axially displace the excitation volume in the sample. A spatial light modulator (SLM) phase-modulated the excitation beam to imprint the vortex $(2 \mathrm{D}$ donut) or annular phase mask (3D donut) onto the wavefront and to compensate for system aberrations. We used a phase-grating in combination with a pinhole in the Fourier plane to omit the polarization fraction that was not phase-modulated by the polarization-sensitive SLM. Two differentially driven electro-optic deflectors (EODs), both located in planes conjugate to the back-focal plane of the objective lens, quickly re-positioned the excitation beams for MINFLUX multiplexing. A piezo-based tip-tilt mirror scanned the illuminating beams in a region of roughly $10 \mu \mathrm{m}$ side length. The scanner was imaged into the back-focal plane of the oil-immersion objective lens using a $4 \mathrm{f}$ system. We mounted the sample on a manual stage integrated with a piezo-driven stage to adjust the sample position. We ensured circular polarization of the excitation beam by placing a $\lambda / 4$ retarder wave plate in the last section of the excitation beam path.

Widefield illumination A polarizing beam splitter combined the point excitation beams with the point activation and widefield excitation. We used another AOTF to select wavelength and power of the widefield illumination (488 nm, $560 \mathrm{~nm}$ or $640 \mathrm{~nm}$ ). Again, we mode-cleaned the beams using a single-mode polarization-maintaining fiber. We focused the beams into the back-focal plane of the objective lens to achieve a collimated illumination of the sample.

Detection The objective lens collected fluorescence light emitted by the sample that was subsequently de-scanned on the tip-tilt mirror and separated from the illumination light using a quad-band dichroic mirror. We selected one of three detection paths by means of electrically driven flip mirrors. We used a camera detection to acquire large sample regions in widefield-mode and to select the MINFLUX imaging regions. For co-aligning the illumination beams and to measure the excitation PSF we used a largearea fiber-based confocal detector (pinhole size several airy units). For MINFLUX acquisition, a true confocal detection (pinhole size $500 \mathrm{~nm}$ in the sample plane) collected the emitted photons to facilitate background suppression. After passing a multi-mode fiber serving as the confocal pinhole, we separated the fluorescence into two spectral ranges using a dichroic mirror with a cut-off wavelength of $685 \mathrm{~nm}$. 
Stabilization We ensured nanometer stability of the sample with an active stabilization system. For measuring the axial sample position, an infrared laser beam $(905 \mathrm{~nm})$ illuminated the sample in total internal reflection mode, so that the displacement of the reflected beam detected on a camera encoded the axial sample position. For measuring the lateral sample position, we used a second camera and a broad wavelength range for illumination (around 950-1000 nm). We acquired dark-field images of one or several scattering nanorods immobilized on the coverslip as fiducial markers encoding the sample position. A proportional-integral-derivative (PID) controller commanded the piezo-stage upon change in the measured sample position. An example measurement of the sample as well as the beam pointing stability is displayed in Figure A.1.

\section{Lasers}

\begin{tabular}{|c|c|c|}
\hline Laser 1 & VFL-P-1500-642 & $\begin{array}{l}\text { MPB Communications Inc., Pointe-Claire, Que- } \\
\text { bec, Canada }\end{array}$ \\
\hline Laser 2 & Cobolt Jive $^{\mathrm{TM}}$ 150-561 & Cobolt AB, Solna, Sweden \\
\hline Laser 3 & 405-50-COL-004 & Oxxius, Lannion, France \\
\hline Laser 4 & Cobolt Jive $^{\mathrm{TM}} 25-561$ & Cobolt AB, Solna, Sweden \\
\hline Laser 5 & LDH-D-C-485 & PicoQuant, Berlin, Germany \\
\hline Laser 6 & LDH-D-C-640 & PicoQuant, Berlin, Germany \\
\hline Laser 7 & LuxX® 905-150 & $\begin{array}{l}\text { Omicron-Laserage Laserproodukte } \\
\text { Rodgau-Dudenhofen, Germany }\end{array}$ \\
\hline Laser 8 & Koheras SuperK Extreme & NKT Photonics, Birkerød, Denmark \\
\hline
\end{tabular}

\section{Beam modulation}

\begin{tabular}{|l|l|l|}
\hline AOTF1 & AOTFnC VIS-TN & AA Sa, Orsay, France \\
AOTF2 & AOTFnC VIS-TN & AA Sa, Orsay, France \\
AOTF3 & AOTFnC 400.650-TN & AA Sa, Orsay, France \\
EOM & LM 0202 P 5W and LIV 20 & $\begin{array}{l}\text { Qioptiq Photonics GmbH Co. KG, Göttingen, } \\
\text { Germany } \\
\text { SLM }\end{array}$ \\
LCOS-SLM X13267-06 & $\begin{array}{l}\text { Hamamatsu Photonics Deutschland GmbH, } \\
\text { Herrsching am Ammersee, Germany }\end{array}$ \\
\hline
\end{tabular}

\section{Scanning}

\begin{tabular}{|l|l|l|}
\hline EODx/y & M-311-A and WMA-300 & $\begin{array}{l}\text { Conoptics Inc., Danbury, CT, USA and Falco Sys- } \\
\text { tems BV, Amsterdam, The Netherlands }\end{array}$ \\
VFL & $\begin{array}{l}\text { KLMS2D0700-00 KTN vari- } \\
\text { NTT Advanced Technology Corporation, Omiya- } \\
\text { focal lens module and AMPS- } \\
\text { 2B200-03 }\end{array}$ & $\begin{array}{l}\text { cho Saiwai-ku, Kawasaki-shi, Japan and Matsu- } \\
\text { sada Precision Inc., Aojicho Kusatsu, Japan } \\
\text { piezosystem jena GmbH, Jena, Germany }\end{array}$ \\
$\begin{array}{l}\text { Tip/tilt } \\
\text { piezo }\end{array}$ & PSH-10/2 and EVD300
\end{tabular}




\begin{tabular}{l|l|l|} 
Piezo stage & P-733.3-DD and E725 & $\begin{array}{l}\text { Physik Instrumente (PI) GmbH \& Co. KG, Karl- } \\
\text { sruhe, Germany }\end{array}$
\end{tabular}

Polarization and beam transport

\begin{tabular}{|c|c|c|}
\hline GT & Glan-Thompson polarizer & B. Halle Nachfl. GmbH, Ber \\
\hline PBS & polarizing beam splitter cube & B. Halle Nachfl. GmbH, Berlin, Germany \\
\hline $\mathrm{BS}$ & beam splitter cube 50:50 & $\begin{array}{l}\text { B. Halle Nachfl. GmbH, Berlin, Germany or Thor- } \\
\text { labs Inc., Newton, NJ, USA }\end{array}$ \\
\hline FC & fiber collimator $60 \mathrm{FC}-*$ & Schäfter and Kirchhoff, Hamburg, Germany \\
\hline$\lambda / 2$ & half wave plate & $\begin{array}{l}\text { B. Halle Nachfl. GmbH, Berlin, Germany or Thor- } \\
\text { labs Inc., Newton, NJ, USA }\end{array}$ \\
\hline$\lambda / 4$ & $\begin{array}{l}\text { achromatic quarter wave } \\
\text { plate }\end{array}$ & Thorlabs Inc., Newton, NJ, USA \\
\hline PM-fiber & $\begin{array}{l}\text { polarization maintaining sin- } \\
\text { gle mode fiber }\end{array}$ & $\begin{array}{l}\text { Thorlabs Inc., Newton, NJ, USA or Schäfter and } \\
\text { Kirchhoff, Hamburg, Germany }\end{array}$ \\
\hline & & on, NJ, USA \\
\hline MM-fiber 2 & multimode fiber M42L02 & Thorlabs Inc., Newton, NJ, USA \\
\hline
\end{tabular}

Lenses and mirrors

\begin{tabular}{|l|l|l|}
\hline Objective & $\begin{array}{l}\text { HC PL APO 100x/1.40 Oil } \\
\text { CS2 }\end{array}$ & Leica Microsystems GmbH, Wetzlar, Germany \\
& $\begin{array}{l}\text { achromatic lens with VIS or } \\
\text { NIR AR coating }\end{array}$ & $\begin{array}{l}\text { Thorlabs Inc., Newton, NJ, USA or Qioptiq Pho- } \\
\text { tonics GmbH \& Co. KG, Göttingen, Germany }\end{array}$ \\
T1-T6 & $\begin{array}{l}\text { telescope } \\
\text { iris diaphragm } \\
\text { ID }\end{array}$ & $\begin{array}{l}\text { mirror on motorized flip } \\
\text { FM }\end{array}$ \\
PH & $\begin{array}{l}\text { pinhole } \\
\text { back side polished mirror }\end{array}$ & Thorlabs Inc., Newton, NJ, USA \\
\hline
\end{tabular}

Dichroic mirrors and filters

\begin{tabular}{|c|c|c|}
\hline DM1 & H 568 LPXR superflat & AHF Analysetechnik GmbH, Tübingen, Germany \\
\hline DM2 & Z500-RDC-XT & $\begin{array}{l}\text { Chroma Technology Corp., Bellows Falls, VT, } \\
\text { USA }\end{array}$ \\
\hline DM3 & Z620SPRDC & $\begin{array}{l}\text { Chroma Technology Corp., Bellows Falls, VT, } \\
\text { USA }\end{array}$ \\
\hline DM4 & ZT405/488/561/640rpc & AHF Analysetechnik GmbH, Tübingen, Germany \\
\hline DM5 & FF685-Di02 & Semrock Inc., Rochester, NY, USA \\
\hline DM6 & FF925-Di01 & Semrock Inc., Rochester, NY, USA \\
\hline F1 & ZET561/10x & $\begin{array}{l}\text { Chroma Technology Corp., Bellows Falls, VT, } \\
\text { USA }\end{array}$ \\
\hline F2 & 488/6 BrighLine HC & Semrock Inc., Rochester, NY, USA \\
\hline F3 & $\begin{array}{l}\text { FF01-842/SP-25 and Quad- } \\
\text { Band } 446 / 523 / 600 / 677 \text { HC }\end{array}$ & Semrock Inc., Rochester, NY, USA \\
\hline
\end{tabular}




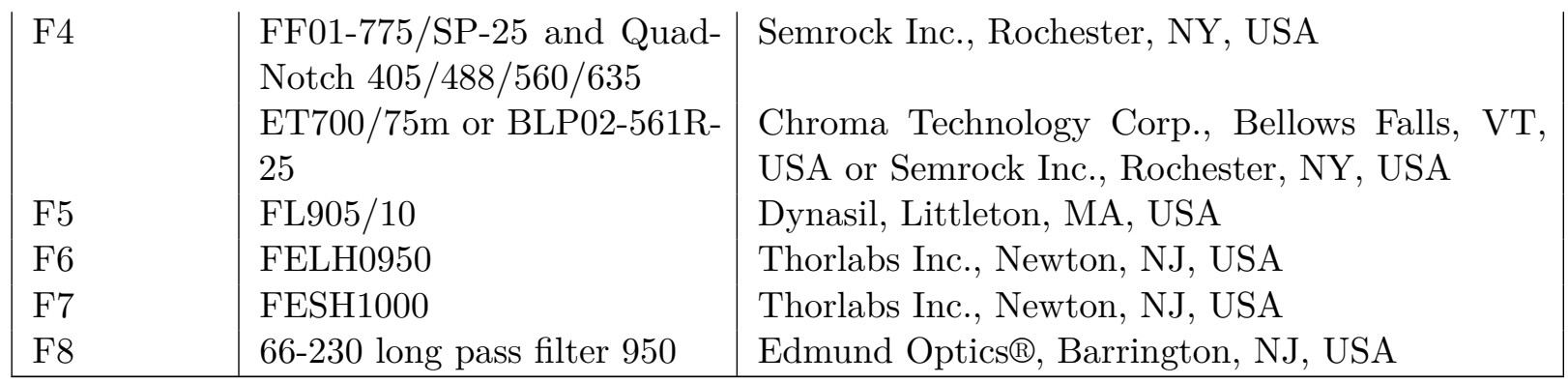

\section{Detectors}

\begin{tabular}{|l|l|l|}
\hline APD 1 & SPCM-AQR-13-FC & Excelitas Technologies, Waltham, MA, USA \\
APD 2,3 & SPCM-AQRH-13-TR & Excelitas Technologies, Waltham, MA, USA \\
Camera 1 & Ixon EMCCD DU897-BV, & $\begin{array}{l}\text { Andor Technology Ltd., Belfast, UK } \\
\text { The Imaging Source Europe GmbH, Bremen, Ger- } \\
\text { Camera 2 }\end{array}$ \\
DMK 22BUC03 & $\begin{array}{l}\text { many } \\
\text { The Imaging Source Europe GmbH, Bremen, Ger- } \\
\text { many }\end{array}$ \\
\hline
\end{tabular}

\section{Computers}

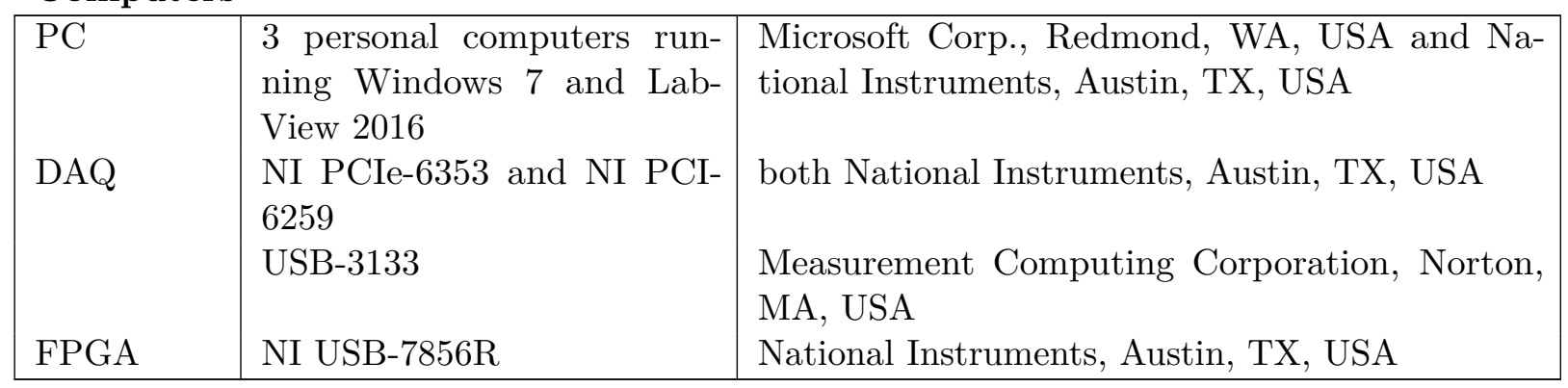

Table 2.1: Hardware components and devices used in the optical microscope displayed in Figure 2.1.

\subsubsection{Experiment control software}

We controlled all hardware components using custom-written software in LabView 2016 (National Instruments, Austin, TX, USA). We additionally used the software Imspector [99] for simple beam- and stage-scanning schemes. Software and hardware components and their connections are schematically displayed in Figure 2.2. We programmed a USB-connected FPGA board to facilitate the iterative MINFLUX acquisition in large fields. Functions of the FPGA board included photon-detection input, live-estimation of the emitter position and control of the beam positions according to predefined parameters and the estimated emitter position. A host program allowed online changes to the FPGA settings and displayed and saved the 


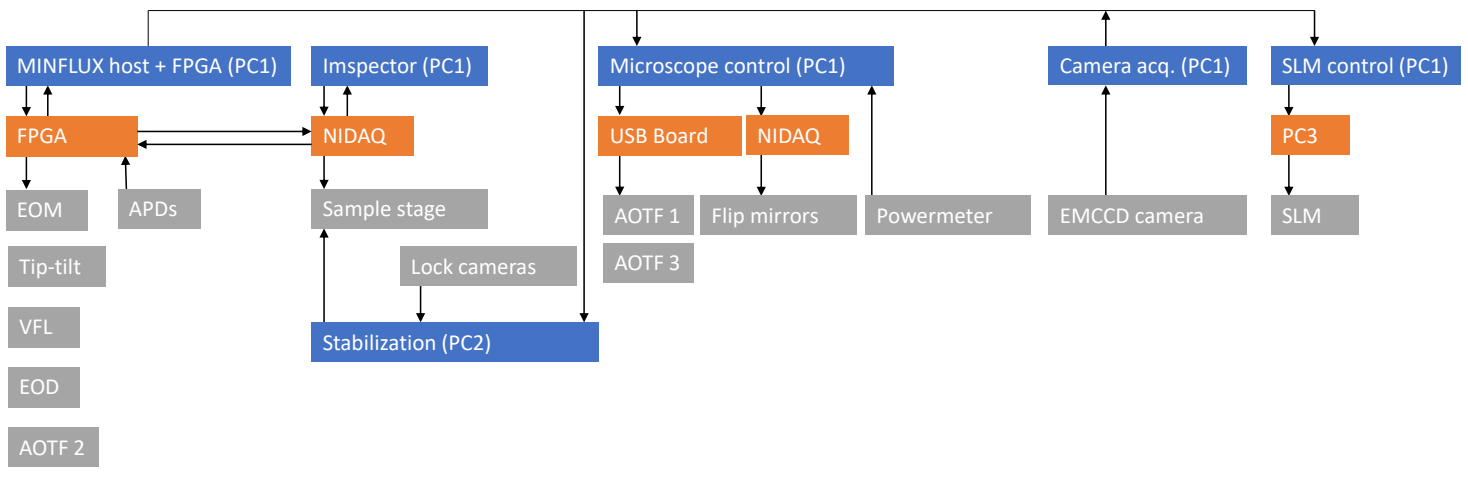

Figure 2.2: Schematic of experiment control software. Software components (blue), hardware interfaces (orange) and hardware components (gray).

photon numbers, beam positions and FPGA settings streamed from the FPGA. The MINFLUX host program communicated with the microscope control, another Labview-written software, to adjust illumination and detection schemes according to the selected settings. Before starting a MINFLUX measurement, we selected a region of interest based on a widefield image acquired in a custom-written camera program. The FPGA host retrieved the scan positions from the camera image. We wrote an SLM program to control the phase distribution displayed on the SLM and to upload the phase image to an additional personal computer (PC) connected to the SLM. We commanded the sample stage using a panel with manual adjustment knobs or via universal serial bus (USB) controlled by the stabilization software.

\subsubsection{Calibrating the beam position}

MINFLUX imaging relies on the precise knowledge of the shape and position of the employed excitation beam. We mapped the voltage applied to the scanners to a physical position in the sample by calibrating the $x y$-scanners for both excitation lines as previously described [6]. We additionally calibrated the electro-optical lens that displaced the excitation beam along the optical axis. We first measured the 3D excitation PSF for different input voltages $U_{\mathrm{EOL}}$ using fluorescent microspheres. To obtain the PSF offset $\Delta z_{\text {Glass }}$ for each input voltage, we modeled the region around the minimum position by a forth-order polynomial. A fit to the quadratic curve $\Delta z_{\mathrm{Glass}}=a_{\mathrm{Glass}} \cdot U_{\mathrm{EOL}}^{2}$ then revealed the parameter $a_{\mathrm{Glass}}$ of the calibration curve. In all 3D MINFLUX experiments, we positioned the beam along the optical axis according to this calibration curve.

As the microspheres were located directly at the coverslip-buffer interface, the calibration curve did not incorporate the axial scaling caused by a refractive index mismatch between the cover glass and the sample $\Delta z_{\text {Sample }}=c_{\text {Sample }} \Delta z_{\text {Glass }}$ [48]. We measured the scaling factor $c_{\text {Sample }}$ with a DNA origami nanoruler labeled with two 
a

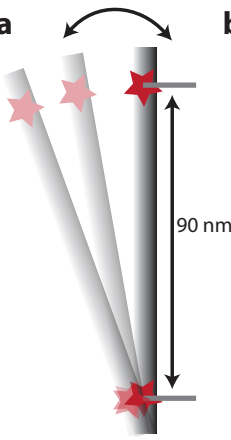

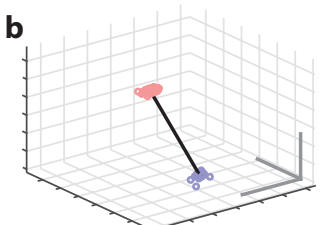

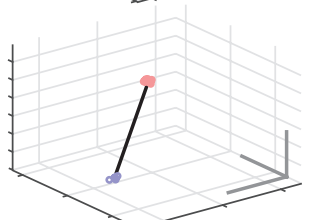

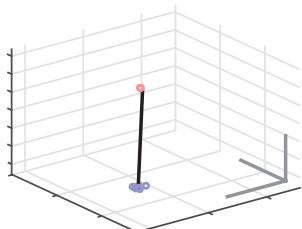

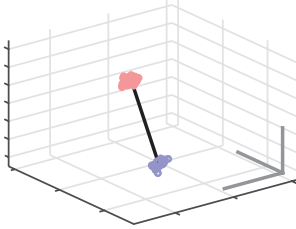

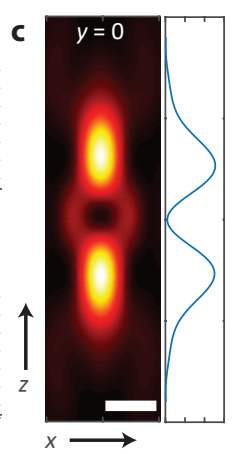

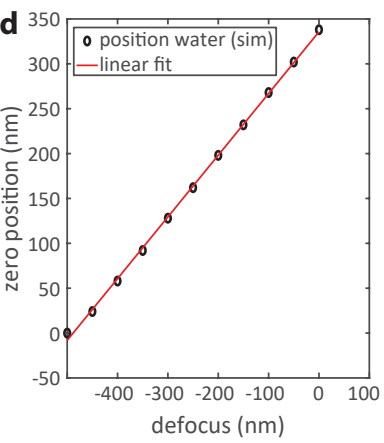

Figure 2.3: Calibration of the beam position along the optical axis. a, deoxyribonucleic acid (DNA) origami ruler with two Alexa Fluor 647 molecules separated by a distance of $90 \mathrm{~nm}$ that can take different orientations when immobilized on a coverslip. b, Fluorophore positions measured with 3D iterative MINFLUX after assigning localizations to single molecules (blue, red). The black line symbolizes the distance between the positions of the molecules. c, Calculated 3D donut with axial profile. The profile was used to determine the axial scaling in simulations. $\mathbf{d}$, Calculated beam position in the sample for different defocus values taking the glass-water interface into account. Scale bars: $50 \mathrm{~nm}$ (b), $200 \mathrm{~nm}(\mathrm{c})$.

Alexa Fluor 647 molecules in a distance of $90 \mathrm{~nm}$ (GATTA-STED 3D 90R - custom, GATTAquant GmbH, Hiltpoltstein, Germany). We prepared the sample as described in Section 2.4.1. After immobilization, the rulers can take a distribution of slightly different orientations (Figure 2.3a). We measured the position of both fluorophores using 3D iterative MINFLUX. Following our standard post-processing steps (see Section 2.3), we extracted localizations with high precision $\left(p_{0}<0.06\right)$. We selected origamis with two fluorophores by extracting measurements with an overall standard deviation of the localizations much larger than the localization precision $(>10 \mathrm{~nm})$, which was the case for about $20 \%$ of the measurements. Using Matlab's kmeans clustering algorithm, we assigned each of the localizations to one of the molecules. We subsequently discarded measurements with a single molecule localization precision larger than $5 \mathrm{~nm}$. An ensemble of resulting measurements is displayed in Figure 2.3b. We calculated the scaling factor $c_{\text {Sample }}=90 \mathrm{~nm}-\sqrt{\Delta x^{2}+\Delta y^{2}} / \Delta z_{\text {Glass }}$ based on the measured projected distances $\Delta x, \Delta y$ and $\Delta z_{\text {Glass }}$. We obtained an average scaling of $c_{\text {Sample }}=0.690 \pm 0.022$, with the error being the standard deviation of 13 measurements. We additionally performed a simulation based on a focus field calculation for high numerical apertures [68] using the realistic beam size and shape as well as realistic objective lens properties. To determine the axial position of the beam in the simulation, we applied an annular phase mask with phase step $\pi$, so that the propagation of the electric fields resulted in a 3D-donut-shaped intensity distribution (Figure 2.3c). To offset the beam by a certain amount, we imprinted a defocus term on the wavefront. We recovered the simulated beam position by determining the 
pixel with lowest intensity within the vicinity of the 3D donut center. To account for uncertainties caused by the finite pixel size, we assumed a linear dependence of the axial shift on the imprinted offset (Figure 2.3d). The simulation delivered a scaling factor $c_{\text {Sample }}=0.6876 \pm 0.0063$ being in excellent agreement with the purely experimental result. We applied a scaling factor $c_{\text {Sample }}=0.7$ in post-processing to correct the axial position estimation in all 3D MINFLUX measurements.

\subsubsection{Aberration correction}

Due to the interference-based generation of the intensity minimum for MINFLUX excitation, optical aberrations in the excitation beam path can deteriorate the zero quality and consequently the intensity contrast that leads to a precise MINFLUX localization. This is especially true for the beam generated from an annular phase mask. For this reason, we measured aberrations induced by the optical system based on a pupil segmentation approach as proposed in [33, 34]. A similar approach has also been applied by other groups to measure sample-induced aberrations [58].

We obtained phase distortions for the entire wavefront by measuring the phase shift of a small area with respect to a reference beam, chosen to be the central region of the very same beam. We inhibited light from any other region of the beam from entering the objective lens by applying the phase-grating on the SLM only for the described regions (see Section 2.1.1). For a beam area of $4 \mathrm{~mm}$ on the SLM we typically distributed overlapping circular pupils of $0.7 \mathrm{~mm}$ diameter, resulting in a total number of 36 pupils. We consecutively applied a phase offset $\Delta \phi_{i}$ to the pupil of interest and detected the interference based on an $x y$-scan of a fluorescent microsphere, expected to deliver an intensity distribution

$$
\begin{aligned}
I^{(j)}\left(\Delta \phi_{i}\right) & =\left|E_{\text {ref }}+E_{\text {pupil }}^{(j)}\right|^{2} \\
& =\left|\sqrt{I_{\text {ref }}} \mathrm{e}^{i \phi_{\text {ref }}}+\sqrt{I_{\text {pupil }}^{(j)}} i\left(\phi_{\text {pupil }}^{(j)}+\Delta \phi_{i}\right)\right|^{2},
\end{aligned}
$$

where the difference between the reference phase and the pupil phase $\phi_{\text {ref }}-\phi_{\text {pupil }}^{(j)}$ is the aberration that we aimed to measure. Without loss of generality we assumed $\phi_{\text {ref }}=0$, so that Equation 2.2 simplifies to

$$
I^{(j)}\left(\Delta \phi_{i}\right)=I_{\text {ref }}+I_{\text {pupil }}^{(j)}+\sqrt{I_{\text {ref }}} \cdot \sqrt{I_{\text {pupil }}^{(j)}} \cdot \mathrm{e}^{i\left(\phi_{\text {pupil }}^{(j)}+\phi_{i}\right)} .
$$

In the experiment, we applied the phase shifts $\Delta \phi_{i} \in[0, \pi / 2, \pi, 3 \pi / 2]$, so that, following a straightforward calculation, we obtained

$$
\phi_{\text {pupil }}^{(j)}=\arctan \left(\frac{I(3 \pi / 2)-I(\pi / 2)}{I(0)-I(\pi)}\right) .
$$


For legibility, we omitted the spatial dependence of all electric fields present in all equations. In the experiments we focused $(z \sim 0)$ and centered the fluorescent microsphere and derived the phase for all pixels $x y$ of the image. Following a phase unwrapping step for avoiding phase jumps within pupils, we fit and subtracted the linear component of the phase in each pupil. We rejected outliers by comparing the median absolute deviation multiplied with a sensitivity factor (typically 4) to the fit error and repeated the fit with rejecting outliers. We selected the phase of an individual pupil to be the value retrieved for one specific pixel. Before interpolating the phase in between the pupils using a thin plate smoothing spline (Matlab function tpaps with smoothing parameter $10^{-3}$ ) we subtracted the linear term of the wavefront based on a linear fit to all pupil phases. Aberration correction delivered an almost fully symmetric excitation PSF as displayed in Figure 3.1.

\subsection{Daily alignment and data acquisition}

We validated the microscope performance on a daily basis using fluorescent microspheres (see Section 2.4.2). We re-examined the co-alignment of activation beam, Gaussian excitation beam and donut-shaped excitation beam and re-adjusted the confocal detection to optimize the overlap with the excitation volume. We imaged the excitation PSF within the area or volume around the donut minimum by scanning over a fluorescent microsphere. For scanning, we used the EODs $(x y)$ and the sample stage $(z)$ respectively. For MINFLUX acquisition, we first mounted the sample as described in Section 2.4.8. We selected a region of interest using widefield illumination and fluorescence detection on a camera. We carefully adjusted the focal plane to ensure full coverage of the structure of interest within the axial range of 3D MINFLUX of roughly $400 \mathrm{~nm}$. When imaging or tracking isolated objects, we determined the center of mass of the fluorescence image of each object on the camera and carried out the MINFLUX scheme at each of the selected objects separately. For imaging large regions, we defined scan positions in a distance of 200-250 nm to ensure homogeneous photo-activation in the whole MINFLUX image area. We scanned either on a rectangular grid or selected an arbitrarily shaped region on the camera image.

Iterative MINFLUX imaging For MINFLUX imaging, we first transferred the fluorescent molecules into a long-lived dark state by scanning the region of interest with a Gaussian-shaped excitation beam. In this off-switching step we applied beam powers of $100-200 \mu \mathrm{W}$ in the back-focal plane. We photo-activated single fluorescent molecules by conditionally applying UV activation light $(405 \mathrm{~nm})$. In a range of about $0.5-5 \mu \mathrm{W}$ we manually adjusted the power of the photo-activation beam depending on the molecular density. The FPGA controlled activation pulses of $0.5 \mathrm{~ms}$ duration, interleaved with probing the presence of a molecule with the beam configuration of 
the first iteration (typically a Gaussian 4-point pattern repeated for $0.1 \mathrm{~ms}$ time or until 40-100 photons were collected). We assumed a molecule to be present if the photon count rate, low-pass filtered with a filter width of $1-10 \mathrm{~ms}$, raised above a predefined threshold of $5-50 \mathrm{kHz}$. As soon as a molecule was detected in the first iteration, the iterative MINFLUX scheme resumed with the succeeding iterations. For this, the molecule was first excited and the emitted photons collected using a predefined TCP and photon number. The FPGA estimated the molecule position and re-centered the subsequent TCP accordingly. The FPGA additionally adapted the excitation beam type and modified the beam separation as defined in the iteration protocol (see Section 1.3). The iterative scheme ended upon collecting a predefined photon number in the last iteration or when the low-pass filtered count rate dropped below an individually defined value in one of the iterations. In the first case, the iterative scheme restarted without illumination with activation light, in the latter case, activation light was conditionally applied until the next molecule was detected. The iterative scheme either stayed at a single scan position until a predefined time period $(0.3-20 \mathrm{~s})$ without emission events had passed or until a predefined number of 10 000-30 000 activation pulses had been applied.

Live position estimators The experimental implementation of iterative MINFLUX requires the on-line estimation of the position of the molecule for subsequent centering of the TCP onto the molecule. Due to the computational complexity, a numerical implementation of the MLE is not suitable for this task. Instead we used the trivial extension of the analytic expression of the mLMSE (Equation 1.38) to three dimensions. We assumed a FWHM of $360 \mathrm{~nm}$ and numerically optimized estimator parameters for a given SBR. For 3D position estimation, the optimization was based on a purely quadratic approximation of the excitation beams. For optimizing the 2D position estimation we provided the experimental PSF. The resulting estimator parameters are listed in Table 2.2. For iterations with Gaussian exposures arranged in a cross-like pattern, we used a modified form of the analytic expression of the 1D MLE as stated in Equation 1.63. We took background into account by using the estimator

$$
\hat{\vec{r}}_{\mathrm{MLE}, \text { Gauss }}=\left(1+\frac{1}{\mathrm{SBR}}\right) \frac{\mathrm{FWHM}^{2}}{8 \ln (2) L}\left[\ln \left(n_{0}\right)-\ln \left(N-n_{0}\right)\right]
$$

where $N$ is the total photon number, $n_{0}$ is the photon number in the first of the two exposures along one spatial dimension, $L$ is the beam separation and SBR is the signal-to-background ratio. We assumed a Gaussian width FWHM $=300 \mathrm{~nm}$ and scaled the resulting estimation with an empirical factor of 0.8 .

Static MINFLUX For the static MINFLUX experiment resulting in the data shown in Section 4.2, we used the acquisition as described in [26]. We adapted the 


\begin{tabular}{|l|c|c|c|c|c|}
\hline Acquisition & TCP & $L \mathbf{( n m})$ & $N_{k}$ & $\beta_{\mathbf{0}}$ & $\beta_{\mathbf{1}}$ \\
\hline \hline Simulations & $\mathrm{G}$ & 300 & 150 & & \\
& $\mathrm{Z}$ & 400 & 100 & optimal & optimal \\
& $\mathrm{D} 7$ & 150 & 150 & optimal & optimal \\
& $\mathrm{D} 7$ & 90 & 150 & optimal & optimal \\
& $\mathrm{D} 7$ & 40 & 450 & optimal & optimal \\
\hline 3D imaging & $\mathrm{G}$ & 300 & 100 & & \\
& $\mathrm{G}$ & 300 & 100 & & \\
& $\mathrm{Z}$ & 300 & 100 & 0.55 & \\
& $\mathrm{Z}$ & 200 & 100 & 0.55 & \\
& $\mathrm{D} 7$ & 150 & 150 & 0.88 & 23.5 \\
& $\mathrm{D} 7$ & 100 & 200 & 0.58 & 31.5 \\
& $\mathrm{D} 7$ & 100 & 10000 & 0.58 & 31.3 \\
\hline 2D imaging & $\mathrm{G}$ & 300 & 40 & & \\
& $\mathrm{G}$ & 300 & 60 & & \\
& $\mathrm{D}$ & 150 & 100 & 1.16 & 8 \\
& $\mathrm{D}$ & 100 & 100 & 0.95 & 8.8 \\
& $\mathrm{D}$ & 50 & 100 & 0.58 & 11.6 \\
& $\mathrm{D}$ & 30 & Inf & 0.4 & 13.5 \\
\hline
\end{tabular}

Table 2.2: Iteration paramters used within this work. The following abbreviations were used for the TCP: G - cross-like TCP with four Gaussian exposures, D - two dimensional four-point triangular pattern with 2D donut exposures, $\mathrm{Z}$ - two exposures with three-dimensional (3D) donut displaced along the optical axis, D7 - seven-exposure cross-pattern in 3D using exposures with $3 \mathrm{D}$ donut. $L$ describes the diameter of the circle connecting the outer exposures. $N_{k}$ is the number of photons collected in iteration $k . \beta_{0}$ and $\beta_{1}$ are the estimator parameters of the mLMSE. All TCPs are visualized in Figures 1.3 and 3.6.

excitation power to obtain count rates of about $100 \mathrm{kHz}$ in order to avoid saturation of the emitting fluorescent molecules.

\subsection{Data analysis}

The data analysis routines used in this work mainly followed the steps that were previously described [6]. First, we segmented the photon count traces to extract the photon counts detected during molecular emission events (Section 2.3.1). For each photon collection, we estimated the position of the molecule (Section 2.3.2) leading to a set of localizations. To omit localizations resulting from an erroneous reaction to background emission or events with impaired convergence in the iterative MINFLUX scheme, we applied localization filters in post-processing (Section 2.3.3). We displayed the localization data in several ways that are detailed in Section 2.3.4. 
For a quantitative assessment of the localization precision, we used two approaches that are described in Section 2.3.5.

\subsubsection{Segmenting the photon count trace}

For MINFLUX imaging, we transferred molecules into a long-lived dark state before starting an acquisition. During acquisition we collected photon count traces for each iteration and multiplex position. Single molecules were photo-activated, leading to photon bursts occurring sequentially and isolated in time. By segmenting the photon count trace, we extracted the molecular photon bursts and removed segments containing only background emission. Based on the extracted molecular photon counts, we subsequently estimated the position of the emitting molecule (see Section 2.3.2). Molecular emission events were characterized by a sharp increase or decrease in the overall photon count rate. We isolated the photon counts detected in the last MINFLUX iteration and assigned all time points to one of the emission states "on" (1), "off" (2) or "blinking" (3) by applying a Hidden Markov model to the sum of counts collected in all TCP positions (Matlab algorithm hmmviterbi). For this, we first estimated the emission rates for molecular emission $\lambda^{(\mathrm{m})}$ and background emission $\lambda^{(\mathrm{bg})}$. We split the median-filtered photon count trace (20-30 samples) at a customarily chosen threshold and calculated the average emission above (molecule) and below (background) the threshold. We assumed Poisson distributions with mean values $\lambda^{(\mathrm{m})}$ and $\lambda^{(\mathrm{bg})}$ as initial emission probabilities in the Hidden-Markov model. The transition probability matrix $T_{i j}$ for a transition from state $i$ to state $j$ was described by

$$
\begin{gathered}
T_{11}=1-T_{12}-T_{13} \\
T_{12}=t_{\mathrm{s}} / t_{\text {off }} \\
T_{13}=0 \\
T_{21}=t_{\mathrm{s}} / t_{\mathrm{on}} \\
T_{22}=1-T_{21}-T_{23} \\
T_{23}=t_{\mathrm{s}} / t_{(\text {blink }, \text { on })} \\
T_{31}=0 \\
T_{32}=t_{\mathrm{s}} / t_{(\text {blink }, \text { off })} \\
T_{33}=1-T_{31}-T_{32}
\end{gathered}
$$

with $t_{\mathrm{s}}=0.1 \mathrm{~ms}$ being the sampling time, $t_{\mathrm{off}}=0.1 \mathrm{~s}, t_{\mathrm{on}}=0.5 \mathrm{~s}$ the estimated on and off-times of the molecule and $t_{(\mathrm{blink}, \mathrm{on})}=1 \mathrm{~ms}$ and $t_{(\mathrm{blink}, \mathrm{off})}=0.1 \mathrm{~ms}$ the estimated blinking on and off times. We run two iterations of the hmmviterbi algorithm, both using the same transition probability matrix. In the second iteration we refined the emission rate distribution based on the states assigned in the first run. To 
avoid unnecessary splitting of single emission events, we merged successive emissions that had been assigned to states "on" or "blinking". To assess the experimental localization precision, we aimed at extracting several localizations for each molecular emission event. To this end, we split the segmented photon traces at a predefined photon number of $N=2000$, usually resulting in 5-10 localizations per event. We further assigned localizations to the same molecule and identifier (ID) if no activation light had been applied in between iteration cycles.

\subsubsection{Position estimation}

We used an MLE to estimate the molecule positions in 2D or 3D data sets from the photon count traces similarly to previously described approaches [6]. In contrast to static MINFLUX realizations, the SBR in iterative MINFLUX depends on the position of the molecule within the TCP and within the confocal volume. As a consequence the SBR varied not only between iterations, but also for successive events from the same molecule, so that we could not extract the SBR directly from the photon count trace. Instead, we estimated the SBR together with the position of the molecule $\vec{r}_{\mathrm{m}}$ using

$$
\begin{aligned}
\hat{\vec{r}}_{\mathrm{m}} & =\underset{\vec{r}_{\mathrm{m}}, \mathrm{SBR}}{\operatorname{argmax}}\left\{\mathcal{L}\left(\vec{r}_{\mathrm{m}}, \mathrm{SBR}\right)\right\} \\
& =\underset{\vec{r}_{\mathrm{m}}, \mathrm{SBR}}{\operatorname{argmin}}\left\{-\ln \left[\mathcal{L}\left(\vec{r}_{\mathrm{m}}, \mathrm{SBR}\right)\right]\right\} \\
& =\underset{\vec{r}_{\mathrm{m}}, \mathrm{SBR}}{\operatorname{argmin}}\left\{-\sum_{j=0}^{K-1} n_{j} \cdot \ln \left[p_{j}\left(\vec{r}_{\mathrm{m}}, \mathrm{SBR}\right)\right]\right\}
\end{aligned}
$$

with the previously defined SBR (Equation 1.66) and multinomial success probabilities $\vec{p}$ (Equation 1.67). We applied the axial scaling factor (see Section 2.1.3) after position estimation with the MLE to obtain the refractive-index corrected position estimate along the optical axis. For the analysis of MINFLUX imaging data, we used a custom-written grid search implementation of the MLE [6]. For the analysis of the experimental data shown in Section 4.2, we employed a simulated-annealing-based optimization using the Matlab function simulannealbnd.

\subsubsection{Localization filtering}

In the iterative data acquisition scheme, we manually defined a count rate threshold to identify the presence of a single molecule. This static threshold definition led to two classes of false-positive iteration events. On the one hand, we misallocated background for emission events because we chose the molecule detection threshold as low as possible to avoid missing out emission events with low emission rates. On the other hand, we reacted to molecules outside the MINFLUX region that were 
unintentionally activated either thermally or by the excitation light.

To omit false-positive events while keeping as many molecular localizations as possible, we applied localization filters in post-processing. The experimental distributions of all filter variables as well as the applied filter values are displayed in Section A of the appendix for all datasets shown in this work.

Emission probability in the central exposure $\boldsymbol{p}_{\mathbf{0}}$ When reacting to background as well as when detecting molecules emitting far outside the MINFLUX region, we expect equal mean counts in all exposures, so that the probability of detecting a photon in the central exposure becomes independent of any molecule position and is distributed around $p_{0}=1 / K$ with $K$ being the number of exposures. Using only localizations with $p_{0}<c / K, c<1$ being a customarily chosen constant, allowed to omit background events. Further restricting the employed $p_{0}$ values by reducing the filtering threshold improved the localization precision of the remaining localizations at the expense of omitting more and more valid localizations. Background emission limited the use of a filter based on $p_{0}$, because for SBR $\rightarrow 0$ also molecular emission events tend towards $p_{0} \rightarrow 1 / K$. This behavior manifests itself in a larger overlap of the $p_{0}$-distribution for true emission and false-positive events.

Distance to TCP center A measure of the event quality that proved to be more robust to a reduction in SBR was the relative distance of the estimated localization to the TCP center in the final iteration, given by

$$
\begin{aligned}
r_{\mathrm{rel}} & =\sqrt{x_{\mathrm{rel}}^{2}+y_{\mathrm{rel}}^{2}+z_{\mathrm{rel}}^{2}} \\
& =\sqrt{\left(x_{\mathrm{est}}-x_{\mathrm{TCP}}\right)^{2}+\left(y_{\mathrm{est}}-y_{\mathrm{TCP}}\right)^{2}+\left(z_{\mathrm{est}}-z_{\mathrm{TCP}}\right)^{2}} .
\end{aligned}
$$

Background events and events of molecules far outside the TCP were estimated close to the edge of the TCP with $r_{\text {rel }} \sim \frac{L}{2}$. By using a filter value $r_{\text {rel }} \sim 0.8 \cdot \frac{L}{2}$, we omitted most of the false-positive events and kept almost all true emission events independently of the SBR.

Estimated position in the MLE grids For false-positive events some MLE estimates failed due to an almost constant or ambiguous likelihood function. In consequence, we omitted events, for which the estimated localization coincided with any of the grid edges in the gridsearch MLE implementation (Exitflag MLE $\neq 0$ ).

Photon number $\boldsymbol{N}$ To guarantee a particular localization precision we omitted localizations with a low photon number in the last iteration.

Emission count rate We discarded events with simultaneous emission from two molecules by defining a count rate threshold. This was only possible for the DNA 
origami dataset, because all cellular acquisitions showed stronger variability in the count rate distribution due to a larger spread of molecule positions in the confocal volume and with respect to the last TCP position.

\subsubsection{Data rendering}

We chose three data rendering strategies to display MINFLUX localizations within this work.

1. We displayed $2 \mathrm{D}$ localizations as simple $2 \mathrm{D}$ histograms with the bin size chosen to be smaller than half the localization precision according to the Nyquist-Shannon sampling theorem (Figure 4.3).

2. For an image-like representation of the data, especially suitable for studying multicolor data, we replaced each estimated position with a Gaussian distribution. Summing up pixel entries for overlapping distributions and normalizing the pixel values delivered the final image matrix $I_{i j}$. We used Gaussian distributions $g(x, y)=\exp \left[-\left(\frac{\left(x-x_{\mathrm{m}}\right)^{2}}{2 \sigma_{x}^{2}}+\frac{\left(y-y_{\mathrm{m}}\right)^{2}}{2 \sigma_{y}^{2}}\right)\right]$ with $\sigma_{x}=\sigma_{y}$ chosen to be the 1D localization precision estimated from all co-aligned localizations. For multicolor data (Figures 4.3 (histogram), 4.4 (Gaussian rendering), 5.2 (Gaussian rendering)), we calculated a red-green-blue color space (RGB) image with the color components being

$$
\begin{aligned}
I_{i j}^{(\text {red })} & =\alpha^{(\mathrm{red})} \cdot I_{i j}^{(\mathrm{CF} 660 \mathrm{C}, \mathrm{CF} 680)} \\
I_{i j}^{(\text {green })} & =\alpha^{(\text {green })} \cdot I_{i j}^{\text {(AlexaFluor647) }} \\
I_{i j}^{(\text {blue })} & =0.7 \cdot I_{i j}^{(\mathrm{CF} 660 \mathrm{C}, \mathrm{CF} 680)},
\end{aligned}
$$

where the factors $\alpha^{\text {(red/green) }}$ were chosen to balance the two imaged molecular species for best visibility.

3. Further, we displayed localization data as scattered dots in a two-dimensional (2D) or 3D coordinate system (Figures 3.8, 3.10, 4.8, 5.9). We chose the size of the dots for best visibility and independently of the experimental localization precision. For some datasets presented, we additionally displayed a surface for visual aid. We estimated the surface shape by clustering the localization using one of the following approaches:

a) Agglomerative hierarchical clustering based on the Matlab functions linkage and cluster using the median to measure the distance between clusters and using an estimated number of clusters (here the number of nuclear pores) in the image as an input (Figure 3.8). 
b) Density-based clustering with the Matlab function dbscan with eps $2 \cdot \sigma_{1 \mathrm{D}}$ and minPts chosen manually for the displayed dataset (Figure 3.10). We additionally split the localization data into two surface elements using the Matlab implementation of the kmeans clustering algorithm.

We estimated the surface based on the cluster centers using the Matlab function tpaps with a smoothing parameter of 0.996-0.999. More elaborate cluster analysis approaches for assigning localizations to single molecules are further described in Section 5.3.

\subsubsection{Estimating the localization performance}

We estimated the localization precision from the MINFLUX images based on localization groups that originated from the same emission event. We obtained several localizations per event by splitting the photons collected within a single MINFLUX iteration cycle (i) or by combining localizations from subsequent iteration cycles that were performed without applying activation light in between (ii).

For estimating the localization precision based on the assigned localization groups or molecule IDs, we determined either the median value of the 1D standard deviation distribution for all molecule IDs (i) or co-aligned all mean localization group positions and determined the spread of single localizations around the co-aligned center based on a 1D Gaussian fit (ii). For both approaches we only considered clusters with at least five localizations.

\subsection{Sample preparation}

Within this work, we used a range of different samples to demonstrate the capabilities of MINFLUX localization. On the one hand, artificial test samples like DNA origamis (Section 2.4.1) or fluorescent microspheres (Section 2.4.2) served for measuring the performance of the setup. In first proof-of-concept measurements, 3D and multicolor MINFLUX images of Nup96, part of the nuclear pore complex (NPC), were acquired in mammalian cells (Section 2.4.3). 3D MINFLUX was used to image PSD-95, part of the post-synaptic protein assembly in hippocampal neurons (Section 2.4.4). Mitochondrial proteins were imaged in several cell types using a staining protocol with directly labeled primary antibodies (Section 2.4.5 and 2.4.6). In all experiments special buffer systems were used for best control of the fluorophores' photophysics (Section 2.4.7), requiring a specialized sample mounting procedure (Section 2.4.8).

\subsubsection{DNA origami}

Annealing For pre-annealing the Rothemund DNA origami template [90] without dye molecules, we mixed the scaffold strands (M13mp18, N4040S, New England 
Biolabs Inc., Ipswich, MA, USA) with 10-fold excess of staple strands (see Table B), both dissolved in Tris-acetate-EDTA (TAE) $1 \mathrm{x}$ containing $27 \mathrm{mM} \mathrm{MgCl}$. We annealed the strands by heating to $85^{\circ} \mathrm{C}$ for $3 \mathrm{~min}$ followed by cooling down in steps of $0.5^{\circ} \mathrm{C} / \mathrm{min}$ until reaching $4^{\circ} \mathrm{C}$. We purified the origami template by adding $15 \%$ polyethylene glycol (PEG) in TAE $1 \mathrm{x}$ containing $0.5 \mathrm{mM} \mathrm{NaCl}$ and $16 \mathrm{mM} \mathrm{MgCl}_{2}$ to the cooled-down solution. While maintaining a temperature of $4{ }^{\circ} \mathrm{C}$, we centrifuged the solution at $13000 \mathrm{rpm}$ for $30 \mathrm{~min}$. After removing excess solution and again supplying $15 \%$ PEG solution, we repeated the centrifugation. We repeated the purification step three times. We stored the DNA origami template in TAE $1 \mathrm{x}$ containing $10 \mathrm{mM} \mathrm{MgCl}_{2}$ at $-20{ }^{\circ} \mathrm{C}$.

Prior to experiments, we annealed the template with the commercially available imaging strands that were 5'-labeled with a fluorescent molecule. We added a 25-fold excess of the labeled strands to the DNA origami template solution and incubated over night at room temperature. We again purified the labeled DNA origami as described above. For the DNA origami used in multicolor MINFLUX imaging (see Figure 4.3), we repeated annealing and purification with a 250x excess of labeled strands.

Immobilization For immobilization of DNA origamis or single DNA strands on a coverslip, we prepared a home-built flow channel based on single-molecule clean coverslips. For cleaning, we sonicated $2-3$ times for 15 min in a $2 \%$ Hellmanex ${ }^{\circledR}$ III solution (Hellma GmbH \& Co. KG, Müllheim, Germany) after placing the coverslips in a home-built teflon holder. To remove remaining detergent, we rinsed the coverslips with Milli-Q water and dried with pressurized air. In a second step, we immobilized gold nanorods on the cleaned coverslips. We diluted the nanorods (A12-25-980CTAB-DIH-1-25, Nanopartz Inc., Loveland, CO, USA) 1:3 in Milli-Q and disposed $30-100 \mu \mathrm{l}$ of the nanorod solution on the coverslips after sonicating the nanorod solution for $10 \mathrm{~min}$. After incubating for less than a minute, we rinsed the coverslips with Milli-Q and dried them with pressurized air. We built the flow channel using double-sided tape (Scotch ${ }^{\circledR}, 3 \mathrm{M}$, Saint Paul, MN, USA) to maintain a flow volume of roughly $15 \mu \mathrm{l}$ between microscope slide and nanorod-coated coverslip. We incubated the channel first with Biotin-BSA $(1.5 \mathrm{mg} / \mathrm{ml}$ in phosphate-buffered saline (PBS), A8549, Sigma-Aldrich, St. Louis, MO, USA) for $10 \mathrm{~min}$, subsequently washing with PBS to avoid binding in solution. We incubated with Streptavidin $(0.5 \mathrm{mg} / \mathrm{ml}$ in PBS, RSTREP-RO, Sigma-Aldrich, St. Louis, MO, USA) for $10 \mathrm{~min}$, followed by another washing step with PBS. We replaced the PBS in the flow channel with folding buffer (TAE $1 \mathrm{x}$ with $10 \mathrm{mM} \mathrm{MgCl} 2$ ) and immobilized the biotinylated and labeled DNA origami diluted to $5-50 \mathrm{pM}$ by incubating for about $5 \mathrm{~min}$. After removing unbound DNA origamis by washing with folding buffer, we filled the flow channel with blinking buffer for MINFLUX nanoscopy or stabilizing buffer for MINFLUX tracking (see Section 2.4.7) and sealed the flow channel using picodent twinsil ${ }^{\circledR}$ speed 
22 (picodent ${ }^{\circledR}$ Dental-Produktions- und Vertriebs-GmbH, Wipperfürth, Germany) to avoid oxygen influx.

\subsubsection{Fluorescent microspheres}

For calibrating the axial beam shift introduced by the VFL, for measuring the PSF of the microscope and for daily alignment of the illumination beams and the confocal detection volume we used references samples with immobilized fluorescent microspheres (FluoSpheres ${ }^{\circledR}, 0.02 \mu \mathrm{m}$, dark red fluorescent; Thermo Fischer Scientific, Waltham, MA, USA). We cleaned the coverslips and immobilized gold nanorods as described in Section 2.4.1. We incubated the coverslips with Poly-L-Lysine $(50 \mu \mathrm{l}$, $0.01 \%$ Poly-L-Lysine solution, Sigma-Aldrich, St. Louis, MO, USA) for 5 min. After sonicating the fluorescent microsphere solution for $10 \mathrm{~min}$, we diluted it to $1: 10^{6}$ in PBS. We incubated the coverslips with $20 \mathrm{\mu l}$ of the bead solution for $1 \mathrm{~min}$ and washed with Milli-Q water. We mounted the coverslip on a home-built flow channel (see Section 2.4.1) that we filled with PBS. We sealed the sample using epoxy glue (Loctite Hysol ${ }^{\circledR}$, Henkel, Düsseldorf, Germany).

\subsubsection{U-2 OS cells for imaging the nuclear pore complex}

For imaging the NPC in mammalian cells we used a cell line available from Cell Line Services (CLS, clsgmbh.de, Nup96-SNAP 293 300444). We handled and prepared the cells as described in [108]. We cleaned the coverslips before seeding the cells by incubating them in a stirred methanol/hydrochloric acid (50/50) solution and rinsing them in water to recover a neutral $\mathrm{pH}$ value before irradiating them with UV light for $30 \mathrm{~min}$. Cells were seeded on clean coverslips two days prior to fixation and incubated at $37{ }^{\circ} \mathrm{C}$ in an atmosphere of $5 \% \mathrm{CO}_{2}$ and $100 \%$ humidity. We used Dulbecco's modified Eagle's medium (DMEM) without phenol red (1180-02, Thermo Fisher Scientific, Waltham, MA, USA), containing 1x MEM NEAA (11140-035, Thermo Fisher Scientific, Waltham, MA, USA), 1x GlutaMAX (35050-038, Thermo Fisher Scientific, Waltham, MA, USA), ZellShield (13-0050, Minerva Biolabs, Berlin, 279 Germany) and $10 \%$ [v/v] fetal bovine serum as growth medium. We prefixed the seeded coverslips in $2.4 \%[\mathrm{w} / \mathrm{v}$ ] formaldehyde in PBS for $30 \mathrm{~s}$, permeabilized in $0.4 \%[\mathrm{v} / \mathrm{v}]$ Triton X-100 in PBS for $3 \mathrm{~min}$ before fixing again for $30 \mathrm{~min}$. We incubated the sample in $100 \mathrm{mM} \mathrm{NH}_{4} \mathrm{Cl}$ in PBS for 5 min to quench autofluorescence of formaldehyde. We applied Image-IT FX Signal Enhancer (I36933, Thermo Fischer Scientific, Waltham, MA, USA) for 30 min prior to staining to enhance specificity of binding. For staining, we left the sample with $1 \mu \mathrm{M}$ BG-Alexa Fluor 647 (S9136S, New England Biolabs Inc., Ipswich, MA, USA) and $1 \mu \mathrm{M}$ dithiothreitol (DTT) in $0.5 \%[\mathrm{w} / \mathrm{v}]$ bovine serum albumin (BSA) in PBS for 50 min at room temperature. Prior to mounting, we washed three times for $5 \mathrm{~min}$ in PBS to remove any unbound dye. To label the center of the pore, we additionally incubated the coverslips with 
wheat germ agglutinin CF680 conjugate (29029, Biotium, Inc., Fremont, CA, USA) diluted to $0.02 \mathrm{\mu g} / \mathrm{ml}$ in PBS containing $1 \%$ BSA for $5 \mathrm{~min}$. We washed at least three times in PBS to remove any unbound dye.

\subsubsection{Primary hippocampal neurons for PSD-95 imaging}

For imaging PSD-95 in hippocampal neurons, we prepared neuron cultures from transgenic PSD-95-HaloTag mice [74] following the protocol described in [18]. The neurons grew 35 days in vitro before we fixed them by supplying formaldehyde solution (4\% in PBS, pH 7.4) for 15 min at room temperature. We washed the cells in PBS before incubating them in $\mathrm{NH}_{4} \mathrm{Cl}(100 \mathrm{mM})$ for $10 \mathrm{~min}$ to reduce autofluorescence background. We permeabilized the neurons using $0.1 \%$ [v/v] Triton X-100 in PBS for $10 \mathrm{~min}$ and incubated with an Alexa Fluor 647-HaloTag ligand $(1 \mu \mathrm{M}$, synthesized in house) for $30 \mathrm{~min}$ at room temperature. We washed several times with PBS before we mounted the coverslips for imaging (see Section 2.4.8).

\subsubsection{Cell culture and staining for mitochondrial imaging}

We cultured human primary dermal fibroblasts (HDFa, ATCC, Manassas, VA, USA) as well as HeLa cells (gift from Prof. Dr. Stefan Jakobs) in DMEM containing $4.5 \mathrm{~g} / 1$ Glucose and GlutaMAX ${ }^{\mathrm{TM}}$ additive (Thermo Fischer Scientific, Waltham, MA, USA) supplemented with $100 \mathrm{U} / \mathrm{ml}$ penicillin and $100 \mathrm{\mu g} / \mathrm{ml}$ streptomycin (Merck Millipore, Burlington, MA, USA), 1 mM sodium pyruvate (Sigma Aldrich, St. Louis, MO, USA) and $10 \%[\mathrm{v} / \mathrm{v}]$ fetal bovine serum (Merck Millipore, Burlington, MA, USA). We equivalently cultured U-2 OS cells (ATCC, Manassas, VA, USA) in McCoy's medium (Thermo Fischer Scientific, Waltham, MA, USA). We seeded cells on coverslips two days prior to fixation and kept them at $37^{\circ} \mathrm{C}$ and $5 \% \mathrm{CO}_{2}$. For imaging Mic10 we used a Mic10-TO HeLa cell line, where a Mic10-FLAG fusion protein replaced the endogenous Mic10. To this end, Mic10 C-terminally fused with a FLAG-T2A-EGFP tag was intergrated into the genome of HeLa Mic10 knockout (KO) cells under the control of a tetracycline-dependent (TetOn) promoter. Induction with doxycycline led to the expression of the fusion protein. The self-cleaving T2A-peptide [94] caused the release of cytosolic enhanced green fluorescent protein (EGFP) as an expression reporter, whereas the FLAG-tagged Mic10 was transported into the mitochondria. Mic10-TO HeLa cells were a gift from Prof. Dr. Stefan Jakobs. We induced Mic10TO HeLa cells for Mic10-FLAG expression by adding DMEM containing $0.025 \mu \mathrm{M}$ doxycycline hyclate (Sigma Aldrich, St. Louis, MO, USA) for $24 \mathrm{~h}$.

Before labeling, we fixed the cells by adding $2 \mathrm{ml}$ of a pre-warmed solution $\left(37^{\circ} \mathrm{C}\right)$ of $8 \%$ formaldehyde in phosphate buffered saline (PBS, $137 \mathrm{mM} \mathrm{NaCl}, 2.68 \mathrm{mM}$ $\mathrm{KCl}$ and $10 \mathrm{mM} \mathrm{Na}_{2} \mathrm{HPO}_{4}, \mathrm{pH} 7.4$ ) to $2 \mathrm{ml}$ DMEM culture medium. After $5 \mathrm{~min}$ we exchanged the solution for $8 \%$ formaldehyde in PBS and incubated an additional 5 minutes. We permeabilized the fixed cells by applying $0.1 \%[\mathrm{v} / \mathrm{v}]$ Triton X-100 
in PBS for 5 minutes and blocked with $5 \%$ [w/v] BSA in PBS/Glycine $(0.1 \mathrm{M})$ for 30 minutes. For staining, we diluted the directly labeled primary antibodies (see Section 2.4.6) in PBS/Glycine $(0.1 \mathrm{M})$ containing $5 \%$ BSA $[\mathrm{w} / \mathrm{v}]$. We incubated the samples for $1 \mathrm{~h}$ at room temperature before washing six times with PBS to remove any unbound labels. For dual-color labeling of Mic10 and Mic19 along with Mic60 we incubated the samples with primary antibodies against Mic10 or Mic19 over night at $4{ }^{\circ} \mathrm{C}$. Afterwards, we incubated the samples with antibodies against Mic60 for $1 \mathrm{~h}$ at room temperature. For labeling of the antibodies we used the protocol as described in Section 2.4.6.

\begin{tabular}{|l|l|l|l|}
\hline Target & Type & Company & Dyes \\
\hline \hline Mic60 & rabbit, polyclonal & $\begin{array}{l}\text { Proteintech, Rosemont, IL, } \\
\text { USA }\end{array}$ & $\begin{array}{l}\text { Alexa Fluor 647, } \\
\text { CF660C }\end{array}$ \\
\hline $\begin{array}{l}\text { Mic10- } \\
\text { FLAG }\end{array}$ & mouse, monoclonal & $\begin{array}{l}\text { Sigma Aldrich, St. Louis, } \\
\text { MO, USA }\end{array}$ & CF680 \\
\hline Mic19 & rabbit, polyclonal & $\begin{array}{l}\text { Atlas antibodies, Bromma, } \\
\text { Sweden }\end{array}$ & CF680 \\
\hline ATPb & mouse, monoclonal & $\begin{array}{l}\text { abcam, Cambridge, United } \\
\text { Kingdom }\end{array}$ & Alexa Fluor 647 \\
\hline
\end{tabular}

Table 2.3: Primary antibodies for mitochondrial imaging. All antibodies were directly labeled as described in Section 2.4.6.

\subsubsection{Direct labeling of primary antibodies}

We labeled primary antibodies directly using amine-reactive crosslinker chemistry at the primary amines of the lysine side chains natively present in the antibodies. First, we purified the antibodies using a centrifugal concentrator (Vivaspin 500, MWVO 10.000, Sartorius, Göttingen, Germany) and re-solved them in PBS (pH 7.4), resulting in an amount of roughly $200 \mu \mathrm{g}$ of antibody at a concentration of $1 \mathrm{\mu g} / \mathrm{\mu l}$. We added $20 \mathrm{\mu l} \mathrm{NaHCO}_{3}(1 \mathrm{M}, \mathrm{pH}$ 8.3-8.5) to the solution and supplied about $20 \mu \mathrm{g}$ of the commercially available 1-Hydroxy-2,5-pyrrolidinedione (NHS) ester derivative of the fluroescent dye (Alexa Fluor 647 NHS Ester, A37573, Thermo Fisher Scientific, Waltham, MA, USA; CF660C Succinimidyl Ester, 92137 and CF680 Succinimidyl Ester, 92139, both Biotium, Inc., Fremont, CA, USA) solved in dimethylformamid (DMF) at a concentration of $10 \mathrm{mg} / \mathrm{ml}$. We protected the solution from light and incubated at room temperature for $1-2 \mathrm{~h}$ while gently stirring. For purification, we pre-washed a gel filtraction column (PD MiniTrap G-25, GE Healthcare Life Sciences, Little Chalfont, Buckinghamshire, United Kingdom) several times with PBS ( $\mathrm{pH}$ 6.5) before adding the antibody solution and eluting again with 
PBS (1x, pH 6.5). A fraction of $200 \mu$ was collected and the protein concentration verified using a micro-volume spectrometer (NanoDrop, Thermo Fisher Scientific, Scientific, Waltham, MA, USA). All antibodies and combinations with dyes employed in this work are listed in Table 2.3.

\subsubsection{Buffers}

For the MINFLUX experiments presented in Chapters 3, 4 and 5, the emission behavior of the fluroescent molecules was controlled using two different buffer systems that are described here.

Blinking buffer for MINFLUX nanoscopy For MINFLUX imaging of samples labeled with Alexa Fluor 647, CF660C or CF680, we used a standard STORM blinking buffer [113]. It contained $0.4 \mathrm{mg} / \mathrm{ml}$ glucose oxidase (G2133, Sigma Aldrich, St. Louis, MO, USA), $64 \mathrm{\mu g} / \mathrm{ml}$ catalase (C100-50MG, Sigma Aldrich, St. Louis, MO, USA), $50 \mathrm{mM}$ TRIS/HCl pH 8.0/8.5, $10 \mathrm{mM} \mathrm{NaCl,} \mathrm{10-200} \mathrm{mM} \mathrm{cysteamine}$ hydrochloride (MEA) (M6500, Sigma Aldrich, St. Louis, MO, USA) and $10 \%$ [w/v] glucose. For DNA origami samples, we prevented dehybridization of the DNA strands by adding $10 \mathrm{mM} \mathrm{MgCl}_{2}$ to the buffer solution.

Buffer for continuous emission in MINFLUX tracking experiments For MINFLUX tracking experiments, we used a reducing and oxidizing system (ROXS) that prevented the blinking and fast photobleaching of the employed molecules [114]. It contained PBS with $10 \%[\mathrm{w} / \mathrm{v}]$ Glucose and $0.5 \mathrm{mg} / \mathrm{ml}$ Trolox (Tertramethylchroman-2-carbocylic acid, 391913-1G, Sigma Aldrich, St. Louis, MO, USA) that we dissolved by stirring and sonicating, followed by a sterile filtering step to remove any undissolved substance. Additionally, the buffer contained $0.6 \mu \mathrm{g} / \mathrm{ml}$ methylviologen-dichloride hydrate (MV, 856177-1G, Sigma Aldrich, St. Louis, MO, USA) and an oxygen scavenging system based on $0.4 \mathrm{mg} / \mathrm{ml}$ glucose oxidase (G2133, Sigma Aldrich, St. Louis, MO, USA) and $64 \mu \mathrm{g} / \mathrm{ml}$ catalase (C100-50MG, Sigma Aldrich, St. Louis, MO, USA) as for the blinking buffer.

\subsubsection{Mounting of cell samples}

For active sample stabilization during MINLFUX acquisition, we also treated all cell samples with fiducial markers (gold nanorods, A12-25-980-CTAB-DIH-1-25, Nanopartz Inc., Loveland, CO, USA). We diluted the supplied solution of gold nanorods 1:3 in single-molecule clean PBS (P4417 Sigma-Aldrich, St. Louis, MO, USA), sonicated for 5-10 min, and incubated the coverslips at room temperature with 100-500 $\mathrm{\mu l}$ of the nanorod solution for 5-15 min. We removed freely floating nanorods by washing the sample at least 3-4 times in PBS. We filled a singewelled microscope slide with the imaging buffer solution, mounted the coverslip on 
top, removed excess of the buffer solution and sealed the samples with picodent twinsil ${ }^{\circledR}$ speed 22 (picodent ${ }^{\circledR}$ Dental-Produktions- und Vertriebs-GmbH, Wipperfürth, Germany) to prevent air exchange. 


\section{IMAGING IN 3D}

As outlined in Section 1.1, several super-resolution methodologies aimed for improving the resolution in all spatial dimensions, while isotropic nanometer-scale resolutions remained difficult to achieve. In this chapter, I present an approach for MINFLUX 3D single-emitter localization based on a beam with a 3D intensity minimum that can be targeted to the molecule in all spatial dimensions. By adopting the lower bounds on the localization precision for 3D MINFLUX, I show that a 3D isotropic nanometer precision can be achieved under realistic conditions. To study the convergence of the position estimators to the theoretical bounds, I numerically test their performance (Section 3.1). I further elaborate on the performance of iterative 3D MINFLUX for imaging micrometer-sized fields of views, demonstrating a further increase in photon efficiency during acquisition, thus theoretically outperforming any standard camera-based localization scheme (Section 3.2). Iterative MINFLUX is used for an experimental validation of 3D MINFLUX by imaging Nup96 and PSD-95 in fixed cells, reaching an isotropic single digit nanometer localization precision over micrometer-sized regions (Section 3.3). I conclude the chapter with a discussion of advantages and limitations of iterative 3D MINFLUX localization (Section 3.4). 


\subsection{Static 3D MINFLUX single emitter localization}

The MINFLUX principle relies on a preferably steep intensity gradient of the excitation beam in the direction of localization (i), on the targeted pointing of the beam coordinate, again in the direction of localization (ii) and on the Poisson statistics of the emitted photons (iii). Keeping these three prerequisites in mind, a minimal 3D MINFLUX implementation needs an intensity gradient in all spatial dimensions (i), ideally showing similar magnitudes, so that an isotropic precision can be achieved, and a spatial displacement of the beam position relative to the sample in all three dimensions (ii). The photon statistics of the emission is independent of the localization's dimensionality and remains unaltered (iii).
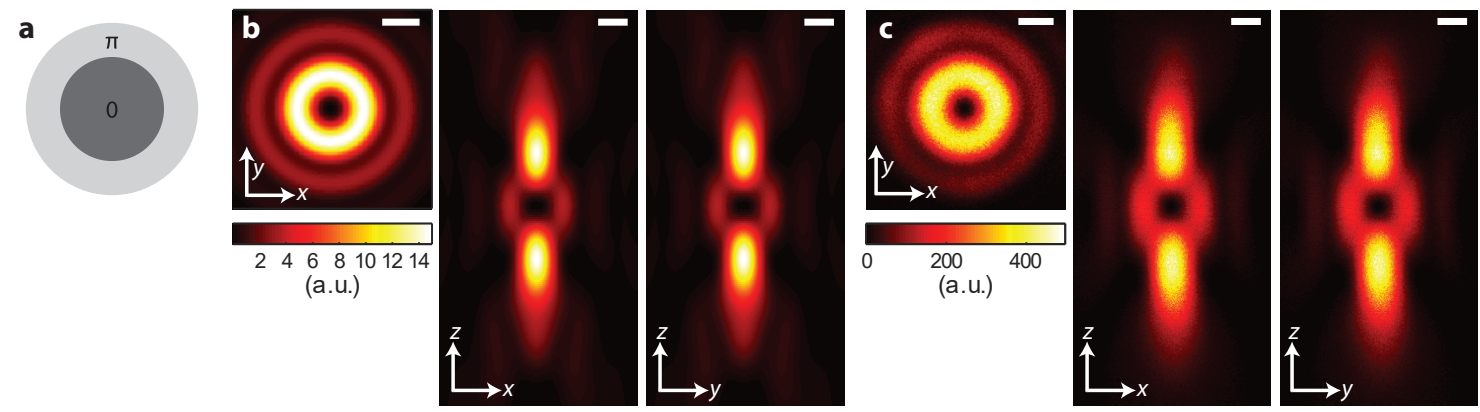

Figure 3.1: Beam shape used for experimental 3D MINFLUX localization. a, Annular phase mask with phase jump of amplitude $\pi$ for the generation of the 3D donut. b, Calculated 3D donut PSF based on an annular phase mask (a) assuming circularly polarized light [68]. c, Experimental beam shape. The wavefront was controlled with an SLM. The beam shape was measured using a fluorescent microsphere. Scale bars: $300 \mathrm{~nm}(\mathrm{~b}, \mathrm{c})$.

For 2D MINFLUX, a donut-shaped beam with small z-gradient and especially vanishing difference and intensity along the line of $x=y=0$ was employed. This beam is unsuitable for a 3D MINFLUX localization. Another donut beam shape readily employed in 3D STED microscopy [63] clearly fulfills the requirements for 3D MINFLUX. The 3D donut-shaped intensity distribution can be generated from a Gaussian-shaped beam by applying an annular phase mask to the beam's wavefront, so that the phase of the inner and outer regions of the beam are shifted by $\pi$ with respect to each other (Figure 3.1a). Fully destructive interference consequently occurs at the focal point with the intensities increasing with growing radial distance (Figure $3.1 \mathrm{~b}$ for a calculated beam shape). We experimentally realized the 3D-donut-shaped beam by introducing an SLM into the excitation beam path (see Section 2.1.1). The SLM displayed an annular phase mask with a $\pi$-jump at a radius at around $70 \%$ of the aperture radius, heuristically optimized for minimal intensity of the excitation PSF at the focal point; for details on the PSF measurement the reader is referred to Section 2.2. We further lowered the residual intensity at the focal point and improved the beam's symmetry by measuring wavefront distortions based on 
a pupil-segmentation approach (see Section 2.1.4). We compensated the wavefront error by applying the opposite of the measured wavefront to the SLM, resulting in a close-to ideal excitation PSF (Figure 3.1c).

To displace the excitation beam in both lateral dimensions, scanning schemes had already been implemented for 2D MINFLUX, including a fast beam positioning with electro-optical deflectors (see Section 2.1.1). Two possibilities allowed for a displacement along the optical axis: a movement of the piezo-driven sample stage or the introduction of a defocus to the beam's wavefront by means of an electrically adjustable lens. We opted for the integration of an electro-optically tunable lens (see Section 2.1.1) for defocussing with less than $10 \mu$ s optical response time, thus not substantially slowing down the MINFLUX data acquisition. Displacing the beam rather than the sample also allowed for a unaltered use of the active stabilization system. We carefully calibrated the amplitude of beam displacement introduced by the tunable lens. We also took the change in beam displacement into account that occurs when focusing into a medium with refractive index different from the coverslip (see Section 2.1.3) [48].

\subsubsection{Theoretical bounds on the localization precision}

With the requirements for a minimal 3D MINFLUX implementation fulfilled, we studied the theoretically expected precision based on the approaches introduced in Chapter 1. We calculated the lower bounds on the localization precision for an unbiased position estimator in all three dimensions and characterized the performance of two position estimators, namely the MLE and the mLMSE.

For a 3D MINFLUX localization, a beam of intensity distribution $I(\vec{r})$ excites a single molecule, where $\vec{r} \in \mathbb{R}^{3}$ is considered to be a three-dimensional vector. The beam is targeted to several positions $\vec{r}_{i}^{(\mathrm{b})} \in \mathbb{R}^{3}$, so that a photon number $n_{i}$ is collected in each exposure $i$ with excitation light. As already introduced in Equation 1.19 for a background-free case and in Equation 1.67 in the presence of background, the probability to detect a photon in a given exposure is described by

$$
p_{i}\left(\vec{r}_{\mathrm{m}}\right)=\frac{\lambda_{i}\left(\vec{r}_{\mathrm{m}}\right)}{\sum_{j=0}^{K-1} \lambda_{j}\left(\vec{r}_{\mathrm{m}}\right)},
$$

where $\lambda_{i}\left(\vec{r}_{\mathrm{m}}\right)$ is the mean Poisson emission at the position of the molecule and $K$ is the number of exposures with excitation light.

We studied the square-root of the diagonal entries of the CRB matrix expressing the $1 \mathrm{D}$ errors of the localization along the coordinate system axes (Equation 1.27). To evaluate the expected deformation of the localization cloud, we considered the isotropy of the 1D errors (Equation 1.29). We examined the lower bounds on the localization precision for different TCP arrangements, including a cross-like 7-point pattern, a tetrahedron-shaped pattern with central exposure and a two-layer triangular 4-point 

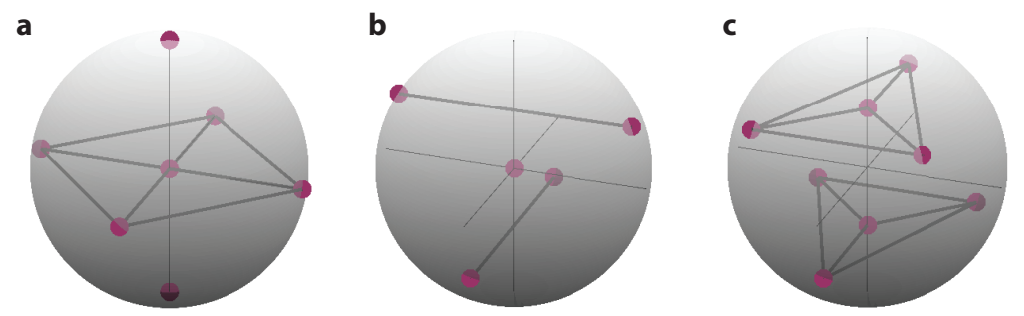

Figure 3.2: TCP arrangements for 3D MINFLUX localization. a, Cross-like 7-point pattern. b, Tetrahedron-shaped pattern with central exposure. c, Two-layer triangular 4-point pattern.

pattern (Figure 3.2). We again assumed that the photo-physical properties of the molecule, the detection efficiency and the background remained constant for all exposures. The conditional probability to detect a set of photon numbers $\left\{n_{i}\right\}$ given a total number of collected photons $N$ consequently follows a multinomial distribution (Equation 1.18). This means that the information-theoretical approach introduced in Section 1.2.2 is applicable. In the case of a three-dimensional localization, the Fisher information on the 3D position of the molecule (Equation 1.21) and consequently also the CRB (Equation 1.26) take a $3 \times 3$ form. We numerically evaluated the CRB for a volume of molecule positions $\vec{r}_{\mathrm{m}}$ around the TCP center resulting in 3D arrays of $1 \mathrm{D}$ standard deviations as defined in Equation 1.27 (Figure 3.3). For the evaluation, we chose a specific TCP arrangement, beam distance $L$, total photon number $N$ and signal-to-background ratio SBR. We reduced the computational load by using a purely quadratic shape of the excitation beam $I(x, y, z)=x^{2}+y^{2}+z^{2}$. Here, we exclusively show the results for the cross-like 7-point TCP (Figure 3.2a) as it outperformed the other patterns with respect to estimator bias, the size of the photon-efficient region and the isotropy of the localizations. Also in the experimental demonstration, the cross-like 7-point TCP was exclusively used.

For experimentally realistic parameters $L=100 \mathrm{~nm}, N=2000$ and SBR $=2$, the theoretical bound on the 1D error $\sigma_{x}$ showed an average value of $1.7 \mathrm{~nm}$ within the volume $\left|x_{\mathrm{m}}, y_{\mathrm{m}}, z_{\mathrm{m}}\right|<25 \mathrm{~nm}$ of molecule positions (Figure 3.3a). In the $y z$-plane, the error $\sigma_{x}$ showed a radially symmetric dependence on the position of the molecule. The best precision was obtained for $x_{\mathrm{m}}=y_{\mathrm{m}}=z_{\mathrm{m}}=0$. An offset in the molecule position along $x$ had a stronger influence on $\sigma_{x}$ than an offset along $y$ or $z$. This is presumably an effect of the non-diagonal entries of the covariance matrix. We observed an equivalent behavior for $\sigma_{y}$ and $\sigma_{z}$ (Figure 3.3b-c). The isotropy $\alpha$ (Equation 1.29) reached $\alpha=0.7$ in volume average. At the TCP center, full isotropy $(\alpha=1)$ was reached. The average 1D error showed full 3D radial symmetry with respect to the center of the TCP (Figure 3.3e).

The numerical evaluation of the lower bounds on the 1D errors demonstrated that for experimentally realistic parameters, a MINFLUX approach based on a cross-like 


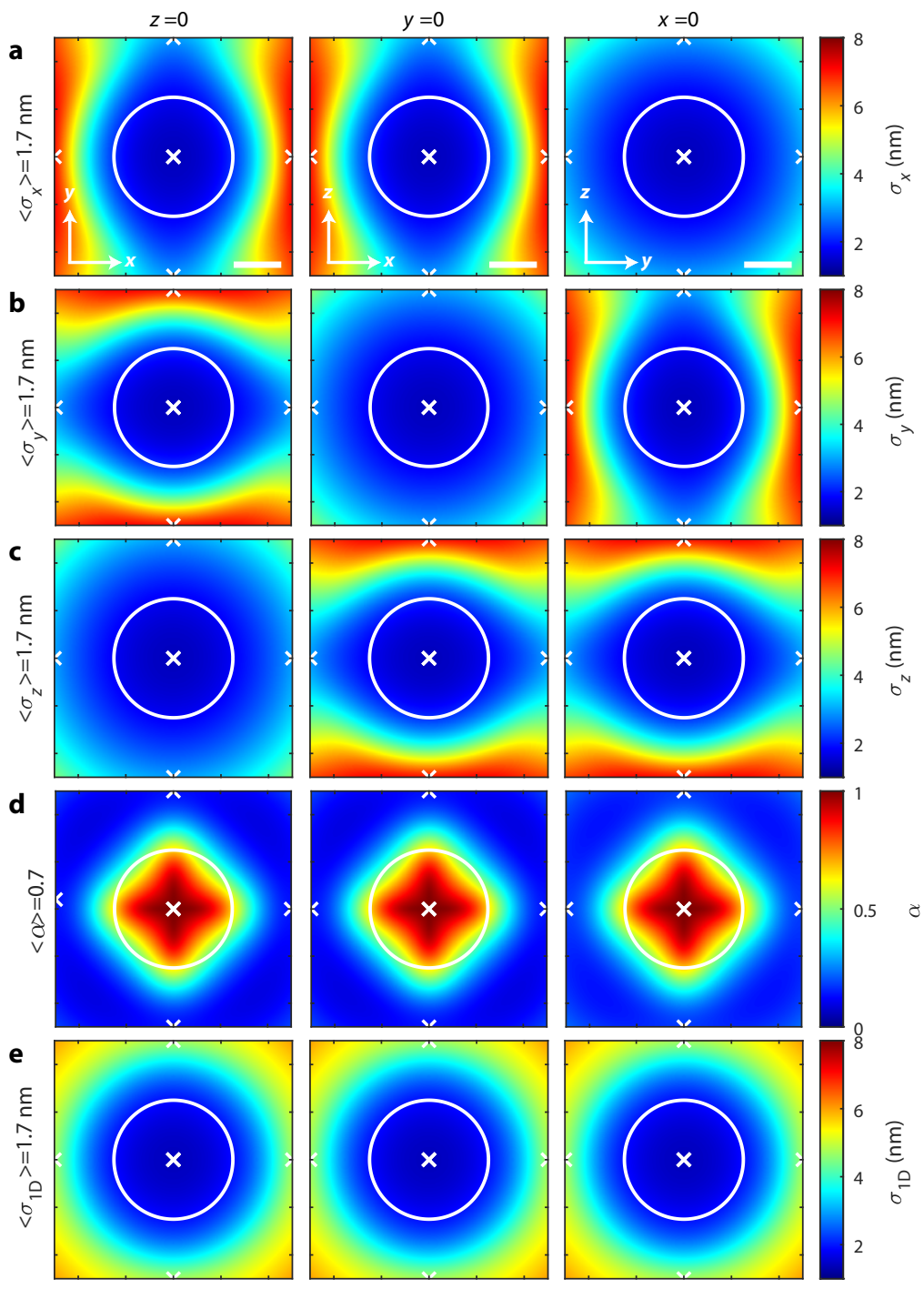

Figure 3.3: Dependence of $1 \mathrm{D}$ errors and isotropy on the position of the molecule for a cross-like 7-point TCP. For the numerical evaluation of the $1 \mathrm{D}$ errors we assumed an ideal quadratic beam shape and used the parameters $L=100 \mathrm{~nm}, N=2000$ and $\mathrm{SBR}=2$. We calculated the errors for molecule positions on a regular 3D grid. a, Spatial distribution of $1 \mathrm{D}$ error $\sigma_{x}$ (color-coded) for three slices $x=0, y=0, z=0$ through the volume of molecule positions. The spatial average $\left\langle\sigma_{i}\right\rangle$ for the volume $\left|x_{\mathrm{m}}, y_{\mathrm{m}}, z_{\mathrm{m}}\right|<L / 4$ (white circle) is displayed on the left. The individual beam targets are indicated with white crosses. $\mathbf{b}$, Same as in (a), but for 1D error $\sigma_{y}$. c, Same as in (a), but for 1D error $\sigma_{z}$. d, Same as in (a), but now showing the isotropy $\alpha$ (color-coded). e, Same as (a), but showing the average 1D error along all three dimensions (color-coded). Scale bars: $20 \mathrm{~nm}(\mathrm{a}-\mathrm{e})$. 
7-point TCP and quadratic excitation beams delivers an almost isotropic precision below $2 \mathrm{~nm}$. The symmetries expected from the TCP as well as the beam shape were clearly reflected in the spatial dependence of the theoretical bounds on the position of the molecule.
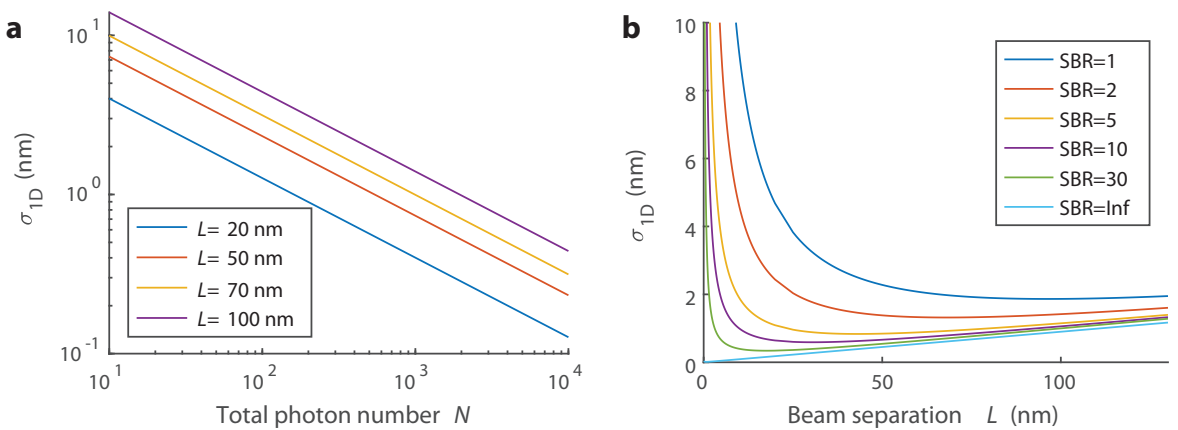

Figure 3.4: Lower bound on the average 1D error for a cross-like 7-point TCP with molecule at the TCP origin. We assumed a purely quadratic beam shape for the evaluation of the 1D error. The given SBR values were obtained for $L=100 \mathrm{~nm}$. a, Dependence of the average 1D error $\sigma_{1 \mathrm{D}}$ on the total number of collected photons $N$ for different beam separations $L$ using $\mathrm{SBR}=50$. $\mathbf{b}$, Dependence of the average $1 \mathrm{D}$ error on the beam separation $L$ in the presence of background using $N=2000$.

We further studied the influence of the total photon number $N$, the signal-tobackground ratio SBR and the beam separation $L$ on the 3D localization performance. The average 1D error for a molecule at the TCP center showed a $1 / \sqrt{N}$ dependence that was maintained for any beam separation and signal-to-background ratio (Figure 3.4a). For a high signal-to-background ratio $\mathrm{SBR}=50$, reducing the beam separation linearly increased the photon efficiency for a given photon number (Figure 3.4a). Further shrinking the TCP size led to a diverging error of the localization with the beam separation for highest photon efficiency depending on the present background level (Figure 3.4b). Lowering the signal-to-background ratio additionally decreased the overall photon efficiency. We defined all signal-to-background ratio values for $L=100 \mathrm{~nm}$, as the effective SBR decreases with smaller beam separations. For smaller beam separations, the signal level is reduced, as the illumination intensity scales quadratically with the distance from the excitation minimum, while the background remains constant. This dependence of the signal level on the beam separation readily explains the behavior observed in Figure 3.4b - with decreasing signal level, the background photons shield the information carried by the emission from the molecule under observation, eventually rendering a localization impossible. Overall, the theoretical bounds on the localization precision in 3D showed a similar dependence on the MINFLUX parameters as already studied for 2D MINFLUX [6]. Even for a very low signal-to-background of 2, the bound on the 1D error fell below $2 \mathrm{~nm}$ for $N=2000$, a range of precision that is inaccessible for localization techniques 
with lower photon efficiency. We further observed that the localization precision was indeed isotropic. The cross-like 7-point TCP decoupled the localization along each spatial dimension as much as possible. Consequently, tuning $L_{x}, L_{y}$ and $L_{z}$ would allow to independently adjust the localization precision along the coordinate axes.

\subsubsection{Position estimators}

The CRB describes the lower bound on the localization precision when using an unbiased estimator. This implies that, depending on the estimator properties (see Section 1.2.3), the lower bound is not necessarily reached when deducing the position of the molecule from photon count numbers. Here, we studied the convergence of MLE and mLMSE to the theoretical bound for increasing photon numbers.
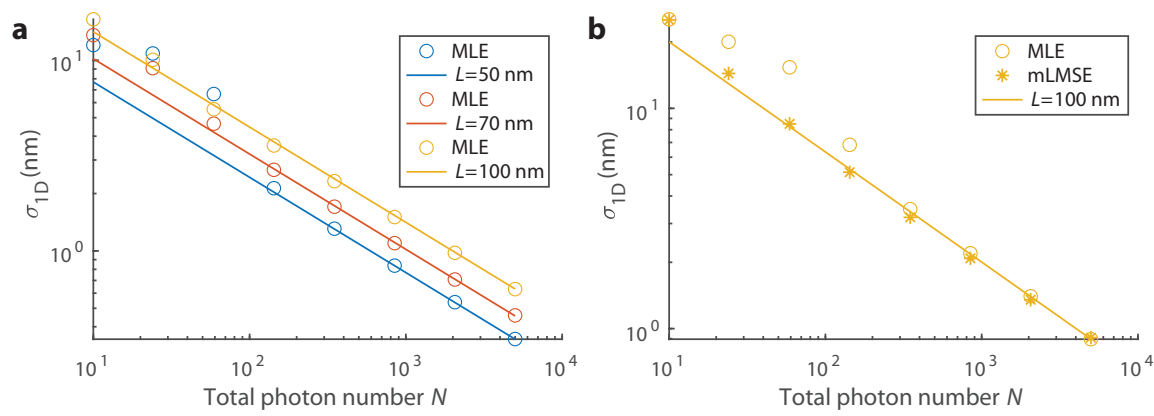

Figure 3.5: Performance of 3D position estimators for MINFLUX. We applied a cross-like 7-point TCP and an ideal quadratic beam shape. For evaluation of the localization error, we generated 10000 sets of multinomial photon count combinations for a molecule situated at the TCP origin. a, Dependence of the lower bound for the 1D error (line) and of the MLE localization error (dots) on the total photon number $N$ using the indicated beam separations $L$ and SBR $=30$. b, Same as in (a), now using SBR $=2$ and $L=100 \mathrm{~nm}$, which are realistic values for measurements in cells. Next to the MLE localization error (dots) the error of the mLMSE is shown (asterisks). The mLMSE parameters were optimized for each photon number by minimizing the localization bias in a spherical volume of radius $0.4 L$.

Due to its beneficial properties stated in Chapter 1, we aimed for using an MLE to estimate the molecule positions in post-processing. The estimator was implemented in a gridsearch optimization approach as described in Section 2.3.2. We numerically evaluated the estimator performance by randomly generating 10000 sets of multinomial photon count combinations (Matlab function mnrnd, see Equation 1.18) for a given total photon number $N$ while assuming the position of the molecule to coincide with the TCP origin. We used SBR $=30$, a value that was previously obtained for the measurement of isolated objects, to study the convergence of the MLE under close-to-ideal conditions. We observed that the spread of the MLE estimates reached the theoretical bound for $N \geq 150$ almost independently of the beam separation $L$ (Figure 3.5a). For $\mathrm{SBR}=2$, a value obtained in cellular measurements later in this chapter, the MLE converged at around $N \gtrsim 500$ (Figure $3.5 \mathrm{~b}$ ). 
Due to its computational complexity and its convergence to the CRB only for higher photon numbers, the MLE is not suitable for live position estimation of the FPGA board.

Instead, we extended the analytic expression of the mLMSE as introduced in Section 1.2.3 to 3D (see Section 2.2) and obtained a numerically simple estimator suitable for online position estimation on the FPGA board. As in previous 2D MINFLUX implementations, we used estimator orders $k=\{0,1\}[6]$. We numerically optimized the estimator parameters $\beta_{0}$ and $\beta_{1}$ by minimizing the overall bias within a spherical volume of radius $0.4 \mathrm{~L}$ for each combination of total photon number $N$, beam separation $L$ and signal-to-background ratio SBR. Again, we randomly generated 10000 multinomial photon count combinations for a molecule at the TCP origin to evaluate the performance of the estimator. Even for $\mathrm{SBR}=2$, the spread of the position estimates reached the CRB already for low photon numbers $N<100$ (Figure 3.5b). We observed that for a molecule at $x=y=z=0$, the mLMSE displayed a slightly smaller spread than expected from the CRB when using an unbiased estimator. This means that the mLMSE was slightly biased under the given conditions. Overall, the mLMSE showed good convergence for experimentally relevant conditions despite its numerical simplicity. The estimator is thus highly suitable for live-position estimation in an iterative scheme or for MINFLUX tracking. For a localization without any bias, the mLMSE would have to be unbiased for each individual molecule position as presented in [6] for the 2D case. When using the MLE with high-enough photon numbers unbiasing is not required due to the estimator's consistency property. For this reason, we used the MLE for 3D MINFLUX position estimation in post-processing.

\subsection{Iterative MINFLUX in 3D}

Up to this point, we studied the theoretical bound on the performance of 3D MINFLUX single emitter localization under the assumption that the TCP shape and extent remained unaltered during photon collection. We confirmed that by using a static TCP, 3D MINFLUX can reach single digit nanometer isotropic localization precision in all three dimensions. We further verified that MLE and mLMSE both reach the $\mathrm{CRB}$ values under experimentally relevant conditions. Similarly to 2D MINFLUX, 3D MINFLUX with a static TCP does not allow for cellular imaging. MINFLUX nanoscopy requires the photo-activation of single fluorescent molecules by illumination with UV light. The photo-activation beam of $405 \mathrm{~nm}$ wavelength, however, covers a diffraction-limited volume. The MINFLUX localization is thus required to work in the full activation volume to avoid loosing molecular localizations. The photon efficiency of the localization improves with shrinking the TCP size, while reducing the TCP size also linearly decreases the size of the FOV. As in the $2 \mathrm{D}$ case, we developed an iterative 3D MINFLUX approach that allowed localizing with high 
efficiency in extended regions by adjusting the TCP shape and size upon more precise knowledge of the molecule's position (see Section 1.3). For this, we predefined TCP shapes and sizes as well as a numbers of photons to be collected in each iteration step. Using the detected photons, we estimated the molecule's position with an mLMSE before re-adjusting the TCP center position and starting the acquisition in the next iteration. In this way we reduced the TCP size over time, rendering photons more informative as the measurement progressed. We determined the beam separation $L$ for the last iteration based on the background level to achieve good photon efficiency (Figure 3.4).

We numerically evaluated the performance of the 3D iterative MINFLUX scheme using three different TCP arrangements and an idealistic signal-to-background ratio (Figure 3.6a). In a first step we assumed Gaussian excitation beams in a cross-like pattern with $L=300 \mathrm{~nm}$ to cover the lateral extent of the activated region of roughly FWHM $=200 \mathrm{~nm}$ spread. We used the available photons to estimate the position of the molecule in both lateral dimensions based on an analytic expression of the MLE (see Section 2.2). In a second iteration, we probed the position of the molecule with a 3D-donut-shaped beam with two exposures displaced along the optical axis. We estimated the molecule's position along $z$ using an mLMSE estimator (see Section 2.2). Together with the first iteration, this delivered a preliminary 3D estimate of the molecule's position. We centered a cross-like 7-point TCP with reduced $L$ onto the molecule to refine the position estimate and consecutively shrank the beam separation after re-centering the TCP while keeping the same TCP type. We numerically evaluated the localization precision in each iteration for an array of molecule positions across the activation volume (Figure 3.6b). We observed a reduction of the localization bias, an improved precision as well as an improved isotropy for subsequent iterations. The final localization precision was spatially homogeneous and isotropic. We assessed the achieved precision in a more quantitative way by studying the spatial average of the lateral and axial localization precision (Figure 3.6c) for the volume shown in Figure 3.6b. The dependence of the lateral and axial precision on the total number of acquired photons decayed faster than $1 / \sqrt{N}$, approximately following a $1 / N^{2}$ behavior for the given choice of iteration parameters (Figure 3.6d). As in the 2D case [43], the exact dependence on the photon number could be tuned by adjusting number of photons, beam type and beam separation in each iteration. We observed that the lateral as well as axial precision of the evaluated iterative MINFLUX scheme surpassed the quantum CRB on the position estimate of any standard camera-based localization approach [5]. The approach even outperformed the theoretical bounds for a conventional coherent $4 \pi$ camera-based detection scheme at about $N_{\text {tot }} \sim 650$ photons. Overall, we obtained an isotropic 3D precision of $\sim 1 \mathrm{~nm}$ using only 1000 photons for all iterations.

The simulation demonstrated that a 3D iterative MINFLUX scheme can deliver spatially homogeneous and isotropic nanometer localization precision within the full activation region in $x y$. The scheme was not able to cover the full axial extent of 
the activation beam. This is because the size of the effective region of the $3 \mathrm{D}$ donut beam between the intensity maxima limits the largest possible beam separation along the optical axis. To overcome this limit, other beam shapes are required.

As in the two-dimensional case, we observed an increase in photon efficiency with progressing iterations that can be attributed to the growing information content of each photon as the TCP is gradually reduced, fundamentally breaking the $1 / \sqrt{N}$ dependence dominating any static localization scheme. In this way, iterative 3D MINFLUX proofed to outperform all standard camera-based acquisition schemes in theory.
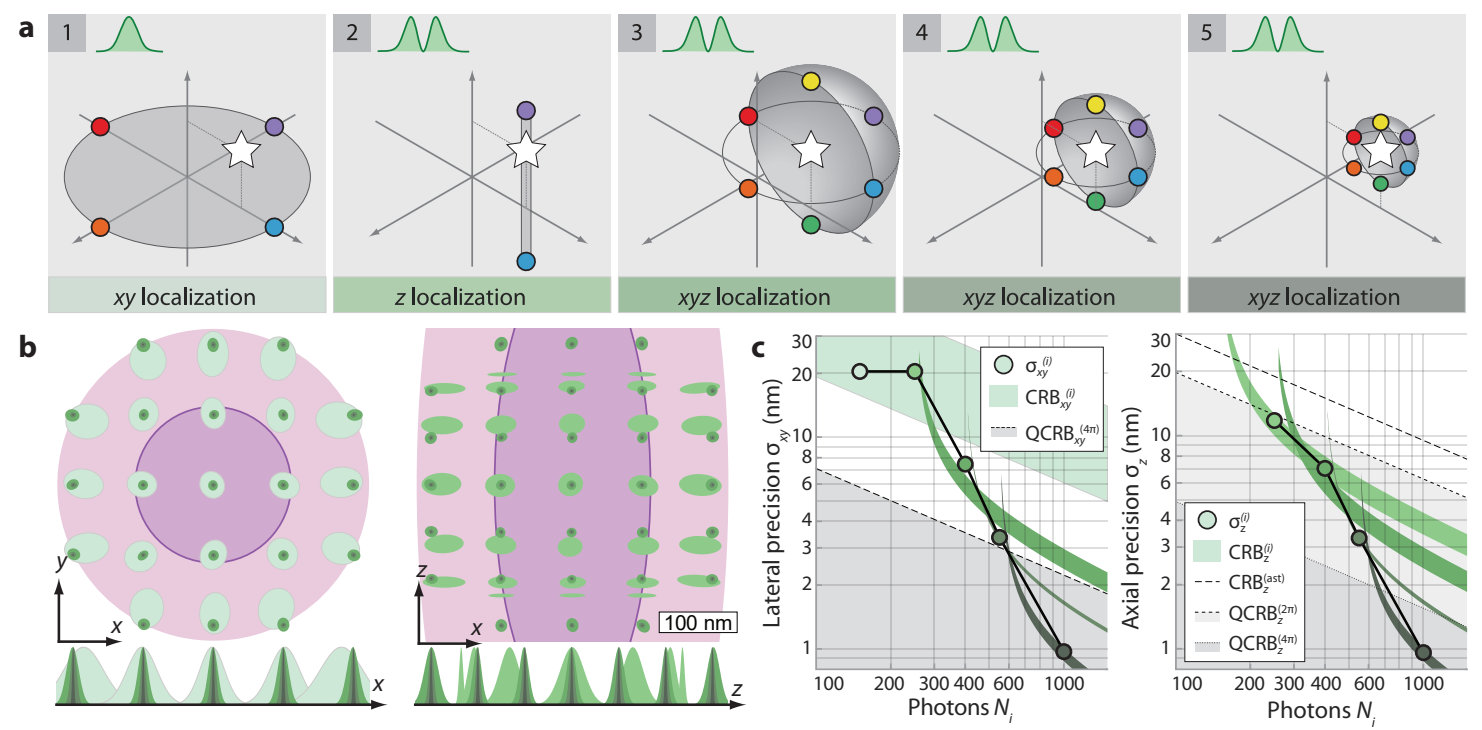

Figure 3.6: Numerical evaluation of iterative 3D MINFLUX. a, Iterative scheme for 3D MINFLUX using a purely 2D localization with Gaussian excitation beams to center the molecule with the 3D donut axis (1), a purely axial localization using the 3D donut beam in two axial positions (2) and 3D localizations using the 3D cross pattern introduced before (3-5). $\mathbf{b}$, Covariance matrices of the estimator for each iteration (green shades) displayed as ellipses of $\mathrm{e}^{-1 / 2}$ level. Several molecule positions within the activated volume were evaluated. The activation beam covers a region with $\mathrm{FWHM}_{x y}=200 \mathrm{~nm}$ and $\mathrm{FWHM}_{z}=600 \mathrm{~nm}$ with $29 \%$ activation probability (purple) and a volume of 2 . FWHM with $86 \%$ activation probability (pink). c, Spatial average of the lateral precision $\sigma_{x y}$ and the axial precision $\sigma_{z}$ of the localizations (green dots) in (b) together with the MINFLUX CRB range for the regions and iterations (green shades). Additionally, the quantum CRB for a coherent camera-based $4 \pi$ configuration is given. For the axial localization, the CRB for camera-based detection using an astigmatism for encoding the axial emitter position and the quantum CRB for a camera-based $2 \pi$ detection are shown. The iteration steps are: $L_{i}=\{300 \mathrm{~nm}$ (Gauss), $400 \mathrm{~nm}, 150 \mathrm{~nm}, 90 \mathrm{~nm}, 40 \mathrm{~nm}\}$, $N_{i}=\{150,100,150,150,450\}$ delivering a total number of $N_{\text {tot }}=1000$ photons. Material from: Gwosch, K.C.*, Pape, J.K.*, Balzarotti, F.* et al., MINFLUX nanoscopy delivers 3D multicolor nanometer resolution in cells, Nature Methods, 2020, Springer Nature. 


\subsection{MINFLUX imaging in 3D}

For proof-of-concept 3D MINFLUX imaging in fixed cells, we combined the iterative MINFLUX scheme (Section 3.2) with the minimal experimental 3D MINFLUX elements, the optimized TCP and reliable position estimators (all described in Section 3.1). We adapted the iteration scheme and the estimator parameters to the cellular background levels, resulting in two additional iteration steps (see Table 2.2), so that we collected a total number of 750 photons, including background photons, before starting the last iteration.
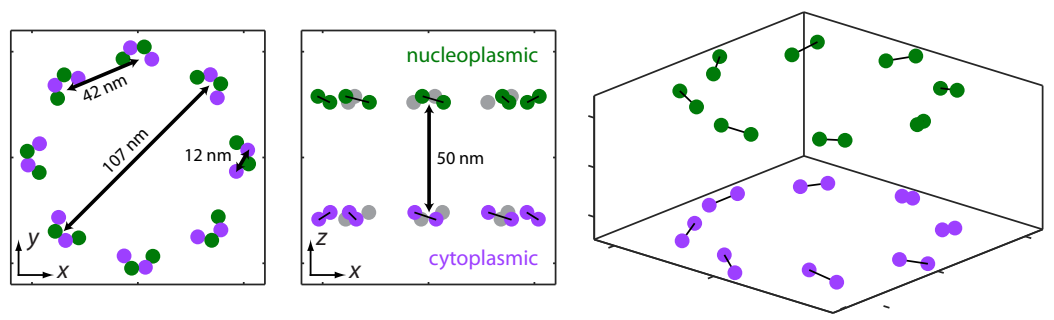

Figure 3.7: Expected distribution of Nup96 in a single nuclear pore complex. The 32 protein copies are arranged in two rings of eightfold symmetry. Nucleoplasmic layer (green), cytoplasmic layer (magenta) and proteins hidden in the background (gray). Distances from [108]. Material from: Gwosch, K.C.*, Pape, J.K.*, Balzarotti, F.* et al., MINFLUX nanoscopy delivers 3D multicolor nanometer resolution in cells, Nature Methods, 2020, Springer Nature.

Imaging of Nup96 For a first experimental demonstration of large FOV 3D MINFLUX imaging in cells, we chose a three-dimensional structure that was wellcharacterized with electron- as well as with optical microscopy, namely the nuclear pore complex (NPC) [115]. We used a U-2 OS cell line that endogenously expressed a fusion protein of the nucleoporin Nup96 to a SNAP tag, a self-labeling protein tag of few nanometers in size [95]. Nup96 is arranged on two rings, one nucloplasmic and one cytoplasmic. The protein distribution shows an eight-fold symmetry with two Nup96 proteins per edge, resulting in a total of 32 Nup96 copies per NPC (Figure 3.7) [108]. After fixation and permeabilization, we labeled the cells with Alexa Fluor 647 (see Section 2.4.3). From an earlier publication using the same cell line and dye for the quantitative characterization of super-resolution microscopy, we expected labeling efficiencies of 40-60\% [108]. We acquired micrometer-sized 3D MINFLUX images as described in Section 2.2 and analyzed the data according to Section 2.3. 3D MINFLUX resolved the eightfold symmetry and the cytoplasmic and nucleoplasmic layers of Nup96 in U-2 OS cells (Figure 3.8). A spline interpolation based on the average locations of single nuclear pores further recovered the shape of the nuclear envelope over an axial range of roughly $300 \mathrm{~nm}$. We quantified the localization precision in 3D MINFLUX imaging based on groups of localizations from a single molecule. 


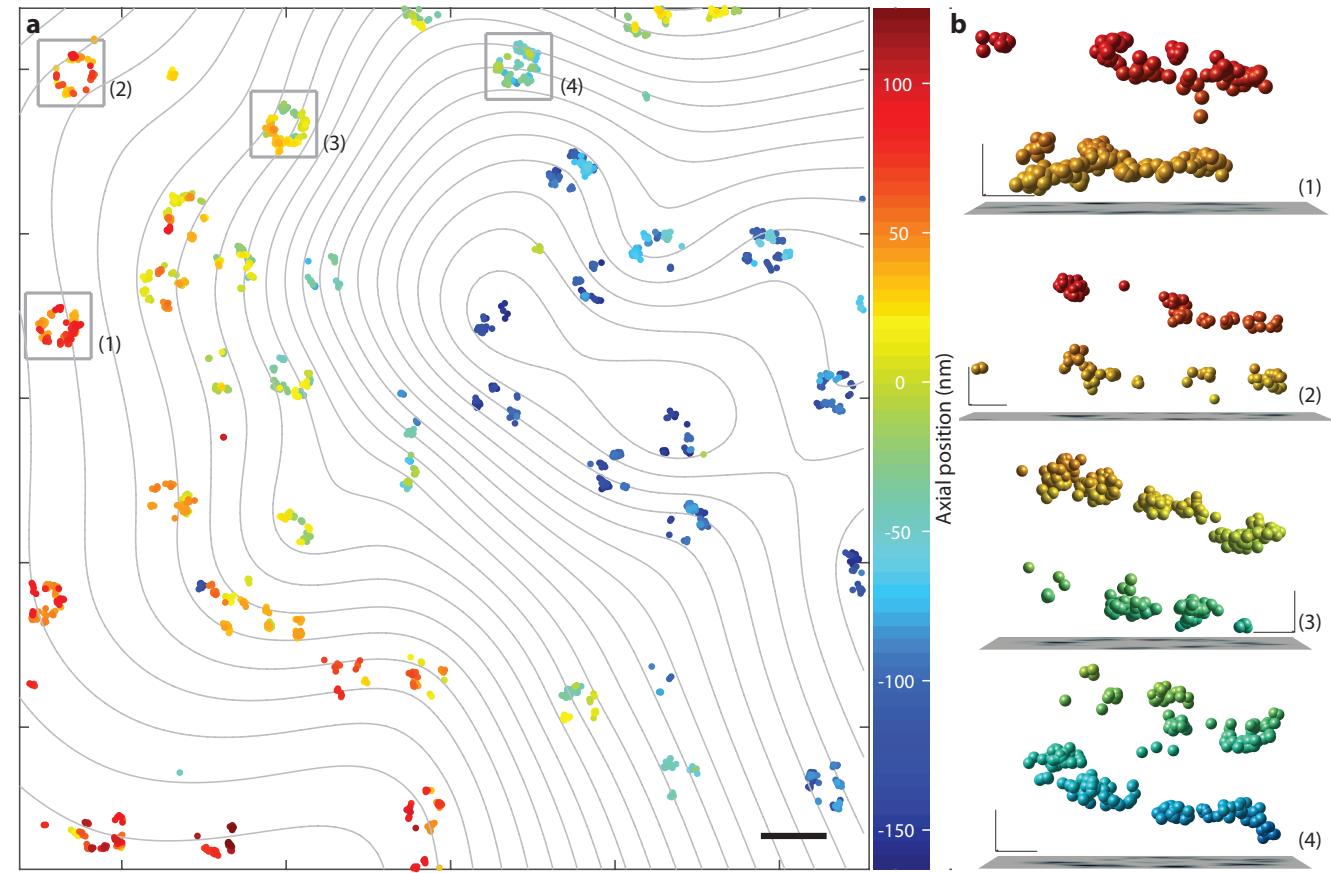

Figure 3.8: 3D MINFLUX image of Nup96 in U-2 OS cells. a, Each Nup96 protein was linked to a SNAP-tag in a stable cell line and stained by reaction of the SNAP-tag with an Alexa Fluor 647-BG substrate. The color represents the axial position estimate for each localization. The size of the scatter dots are unrelated to the localization precision. The contour lines indicate the shape of the nuclear envelope, obtained from clustering single NPC units and spline interpolating the cluster centers. b, Side view of marked regions in (a) showing the distance between the nucleoplasmic and cytoplasmic layers of Nup96. Scale bars: $200 \mathrm{~nm}$ (a), $20 \mathrm{~nm}$ (b). Material from: Gwosch, K.C.*, Pape, J.K.*, Balzarotti, F.* et al., MINFLUX nanoscopy delivers 3D multicolor nanometer resolution in cells, Nature Methods, 2020, Springer Nature.
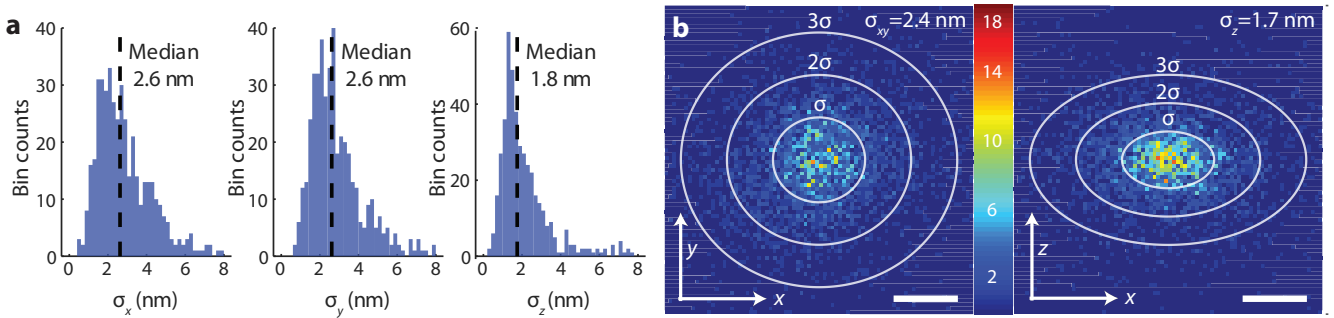

Figure 3.9: Estimating the experimental localization precision in 3D MINFLUX imaging. We used the dataset shown in Figure 3.8. a, 1D histograms of standard deviations for each group of localizations assigned to a single molecule. Photon counts from single emission events were split after $N=2000$ photons in the last iteration, delivering several localizations for a single emission event. Emission events with less than 5 localizations were discarded. $\mathbf{b}$, Co-aligned localizations for all emission events by subtracting the average position of each localization group from the individual localizations. The errors were obtained from a 1D Gaussian fit to the corresponding 1D distributions ( $\sigma$-value and white ellipses of major axis length $2 \sigma, 4 \sigma$ and $6 \sigma$, respectively). Scale bar: $5 \mathrm{~nm}$ (b). Material from: Gwosch, K.C.*, Pape, J.K.*, Balzarotti, F.* et al., MINFLUX nanoscopy delivers 3D multicolor nanometer resolution in cells, Nature Methods, 2020, Springer Nature. 
We grouped localizations that we obtained from a consecutive train of molecular emission without application of activation light in between (see 2.3.5). We artificially split the photons after 2000 counts in the last iteration, so that many molecular emission events delivered 5-10 localizations. We excluded localization groups with less than 5 elements from further analysis. We estimated the localization precision based on two measures. First, we calculated the median of standard deviations of singe localization groups (Figure 3.9a), resulting in $\sigma_{x y} \sim 2.6 \mathrm{~nm}$ and $\sigma_{z} \sim 1.8 \mathrm{~nm}$. Secondly, we co-aligned the mean position of all localization groups and determined the spread of the resulting distribution based on a 1D Gaussian fit, delivering slightly better localization precision values (Figure 3.9b). We should keep in mind, however, that our localization precision estimate based on co-aligned localizations is slightly biased towards lower values due to the low sample size for the estimation of the mean position for individual localization groups.

Imaging of PSD-95 in cultured neurons We further studied the applicability of 3D MINFLUX for imaging synaptic proteins in dissected hippocamapal neuron cultures. We chose PSD-95 as a target, which is part of an electron-dense region of the post-synapse. PSD-95 is thought to play a key role in the anchoring and distribution of glutamate receptors, thus influencing the transmission strength at the level of single synapses $[32,73]$. The protein was target of a number of super-resolution studies, most of them limited to high-resolution 2D imaging [72]. Observing only the projection of synapses that are randomly oriented in 3D, however, can lead to artifacts. For imaging PSD-95 with 3D MINFLUX we used neuron cultures from transgenic mice expressing a PSD-95-Halo-tag fusion protein [74], so that we could label the C-terminus of PSD-95 with Alexa Fluor 647 after fixation (see Section 2.4.4). We observed MINFLUX localizations in confined clusters at roughly $40 \mathrm{~nm}$ nearestneighbor distance that were distributed on a slightly curved surface of $100-400 \mathrm{~nm}$ side length (Figure 3.10). We obtained a 1D standard deviation of 4-6 nm for the localizations in a single cluster. Each cluster contained a median number of $\sim 30$ localizations. In combination with the experimental 1D localization precision of $2-3 \mathrm{~nm}$ (Figure 3.10c), the high number of localizations per cluster suggests that a single cluster was composed of more than one Alexa Fluor 647 molecule. This result indicates that C-termini of several PSD-95 proteins might be spatially aligned.

\subsection{Summary and discussion}

In this chapter, we outlined the theoretical background for 3D MINFLUX single molecule localization by studying the Cramér-Rao lower bound on the localization precision for different MINFLUX imaging parameters, namely total photon number $N$, beam separation $L$ and signal-to-background ration SBR. We showed that a nanometer isotropic 3D localization precision can be reached within a spherical 
a
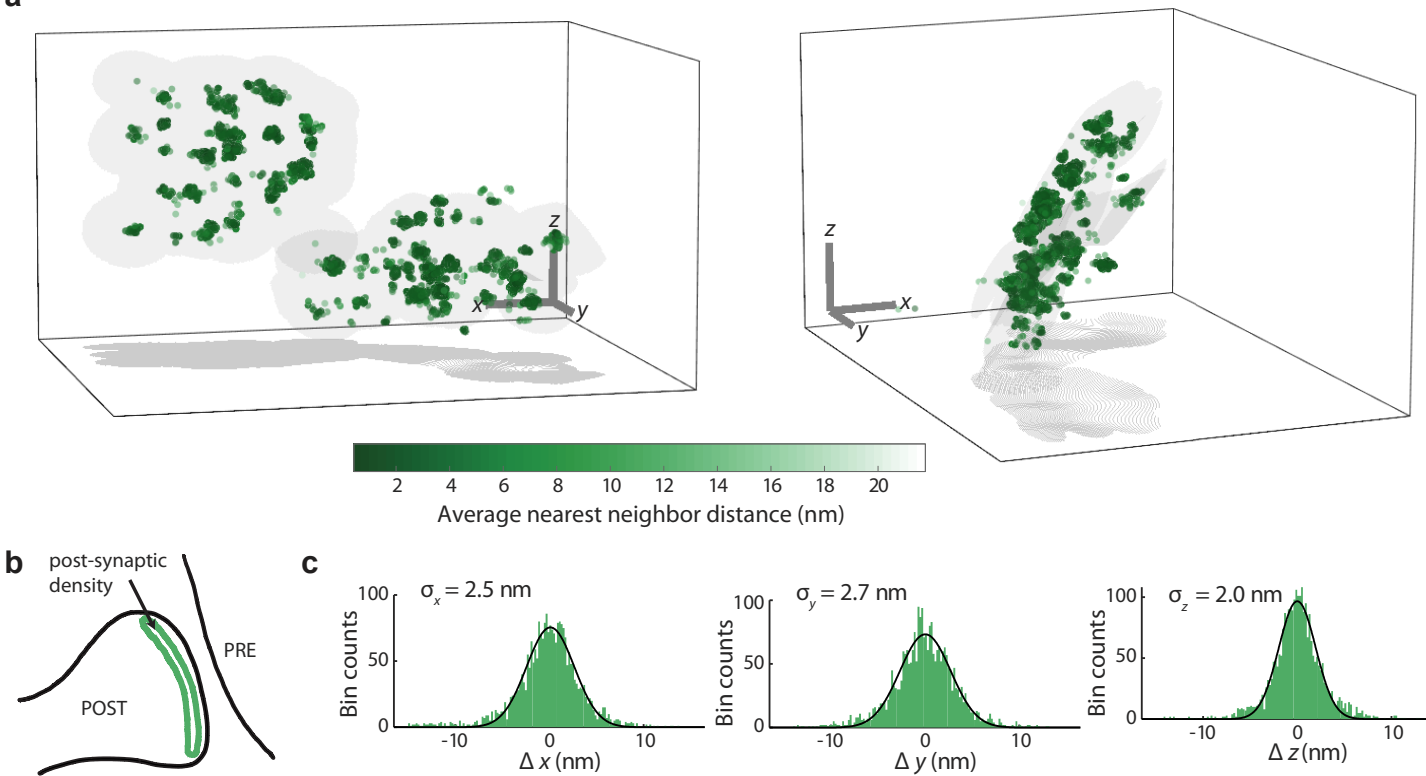

C
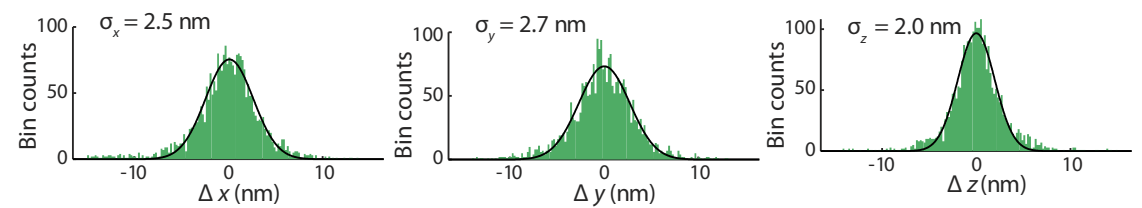

Figure 3.10: 3D MINFLUX image of PSD-95 in cultured hippocampal neurons. a, Two perspectives of a 3D MINFLUX image of PSD-95 labeled with Halo-Alexa Fluor 647. The average distance to the next five nearest neighbors, encoding the density of localizations is indicated by color. A spline interpolation based on the localization cluster centers led to two independent surfaces shown as gray surfaces and contour line projections onto the lower $x y$ bounding plane. $\mathbf{b}$, Schematic drawing of the synapse with the post-synapse being part of the dendritic spine and the pre-synapse located at the axon. $\mathbf{c}, 1 \mathrm{D}$ histograms of localization distributions in MINFLUX acquisition of PSD-95 after co-aligning localizations according to the estimated average position of the molecule. 1D Gaussian fits (black lines) deliver a localization precision of $\sigma_{x, y, z}=2-3 \mathrm{~nm}$. Scale bar: $100 \mathrm{~nm}$ (a). Material from: Gwosch, K.C.*, Pape, J.K.*, Balzarotti, F.* et al., MINFLUX nanoscopy delivers 3D multicolor nanometer resolution in cells, Nature Methods, 2020, Springer Nature.

volume of $25 \mathrm{~nm}$ when using only $N=2000$ photons. This turned out to be true even in the presence of strong background delivering SBR $=2$ at the TCP origin for a beam separation of $L=100 \mathrm{~nm}$. We showed that the estimators used for the experimental analysis (MLE) and for the re-centering of the TCP during the experiment (mLMSE) reached the CRB under the experimental conditions.

We demonstrated that iterative 3D MINFLUX can lift the constraint in FOV of the static 3D MINFLUX approach, so that a homogeneous and isotropic 3D localization precision is achievable even in micrometer-sized regions. The image can be assembled by scanning the TCP and the activation beam over the sample. The proposed iterative 3D MINFLUX scheme outperformed all standard camera-based approaches in theory, including coherent two-objective arrangements, when collecting more than $N_{\text {tot }}=650$ photons in all iterations. The photon efficiency was increased in each iteration, so that the localization precision did not follow a $1 / \sqrt{N}$ dependence 
anymore, but decayed approximately with $1 / N^{2}$.

We applied 3D MINFLUX to imaging micrometer-sized regions of the nuclear envelope, where Nup96, part of the NPC, was labeled with Alexa Fluor 647. We achieved an experimental precision of $1.5-3 \mathrm{~nm}$ in all spatial dimensions, demonstrating that a single digit nanometer localization precision is feasible when using iterative 3D MINFLUX for cellular imaging. We achieved a slightly better localization precision along the optical axis, which arises most likely due to the refractive index mismatch at the coverslip-buffer interface, leading to an effective reduction in beam separation along the optical axis, directly relating to a higher localization precision.

Current limitations in performance are mostly related to the experimental implementation. The axial range that can be covered is confined by the finite defocus that the varifocal lens (VFL) can apply to the excitation beam, leading to a focal displacement of maximally $300-400 \mathrm{~nm}$, so that not all molecules within the activation volume can be reached. Different approaches could be used for defocussing, including stage scanning or the use of mechanically actuated mirrors, both at the expense of acquisition speed. A further restriction arises from the beam shape in the first axial localization, as the region of high gradient between the intensity maxima of the 3D donut beam does not allow to cover the full activated region. Using other beam shapes, for example tilted 2D donut beams, could also be beneficial for reducing background emission, partially arising due to the strong axial lobes of the employed 3D donut. We should note that we did not reach the CRB in the experimental implementation of 3D MINFLUX. This can have different reasons, one being the uncertainties in the beam shape. For the analysis we assumed an ideal quadratic beam shape, which neglects the $x^{4}$ dependence of the realistic 3D donut. Further, we noted a change in the experimental PSF over hours, presumably being related to charges accumulating in the electro-optical crystals used for defocussing. Also here, different approaches for axial scanning might reduce experimental errors. An approach for measuring the 3D PSF should be developed, so that the accuracy of the 3D MINFLUX measurement can be further increased. Another reason for the deviation of our experimental precision from the CRB can emerge from the simultaneous estimation of molecule position and SBR as described in Section 2.3.2 that is not covered in the CRB calculation.

Regarding biological applications, imaging speed is a major obstacle. The imaging speed hardly differs between 2D and 3D MINFLUX imaging, but scales with the number of molecules within the FOV as well as with the size of the FOV itself. The number of molecules plays a role as only one molecule is imaged at a time with additional waiting times arising from a tunable activation probability that is chosen to avoid simultaneous activation of several molecules. The development of parallelization approaches promises to strongly increase imaging speeds. In contrast to STED imaging, the employed beam powers are rather low, so that a parallelization can be achieved similarly to previously proposed reversible saturable optical fluorescence transitions (RESOLFT) imaging schemes [14, 75]. 



\section{MULTICOLOR LOCALIZATION}

In the previous chapter, I established a 3D MINFLUX approach for imaging micrometer-sized volumes in cells. I dedicate the present chapter to multicolor MINFLUX localization for measuring inter-species distances. I present two approaches - one suitable for MINFLUX nanoscopy (Section 4.1), the other allowing MINFLUX tracking experiments (Section 4.2).

In MINFLUX nanoscopy, fluorophores with similar excitation spectra can be distinguished based on a subtle difference in their emission spectra. The difference can be observed by spectrally splitting the emission light onto two or more detectors. I elaborate on the assignment of the emission events to one of the imaged species based on the photon numbers detected on each spectral channel and demonstrate the viability of this approach for nanometer-scale multicolor MINFLUX imaging. Acquiring a two-color 2D MINFLUX image of a custom-made DNA origami structure, I achieved a localization precision of around $1 \mathrm{~nm}$. In multicolor 3D cellular imaging of the NPC, I measured an isotropic precision below $4 \mathrm{~nm}$ for both molecular species. For multicolor MINFLUX tracking, two or more fluorophores are required to emit simultaneously so that their positions and their distance can be monitored over time. I present a theoretical description of the photon collection process for simultaneously emitting molecules and discuss the advantages and limitations based on a numerical evaluation of the localization performance and a first proof-of-concept measurement. I found that the two emitters can be localized simultaneously with nanometer precision, recovering the expected distance of $24 \mathrm{~nm}$ between two molecules on a custom-made DNA origami structure. The localization relied on the reproducibility of the photo-physical properties of the dyes, however, which poses an additional challenge for tracking applications. I conclude the chapter with a discussion of the results on multicolor MINFLUX localizations. In the next chapter, I then apply multicolor 3D MINFLUX imaging for studying the distribution of proteins within mitochondria (Chapter 5). 


\subsection{Multicolor MINFLUX for imaging applications}

Numerous approaches have been presented for multicolor super-resolution microscopy. One class of approaches uses a difference in molecule kinetics as for example the fluorescence lifetime [107] or the switching characteristics or binding/unbinding kinetics in DNA-PAINT $[60,118]$ to distinguish molecular species. It is also possible to discriminate groups of dyes based on a difference in their molecular spectra, either in absorption [23], by using activator-reporter dye pairs [8] or in the fluorescence emission [125].
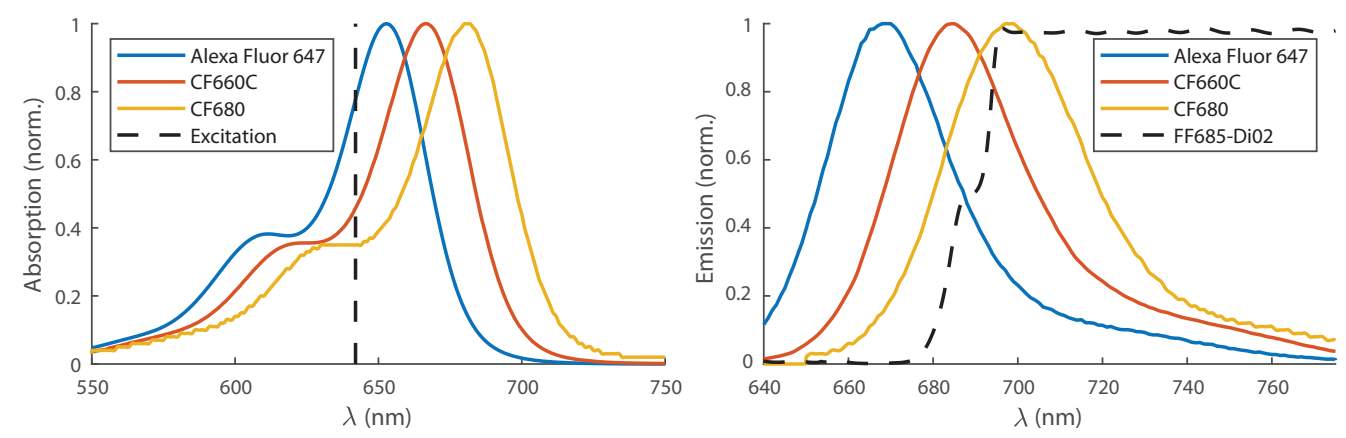

Figure 4.1: Spectra of employed dyes and dichroic mirror. Absorption (left) and emission (right) spectra for the three fluorescent dyes employed in this work. The excitation wavelength $(\lambda=642 \mathrm{~nm}$, dashed line) is shown together with the absorption spectra. The transmission spectrum of the dichroic mirror (dashed line) used for color separation is shown together with the emission spectra. All spectra were obtained from https://searchlight.semrock.com/.

By using a difference in the emission spectra, exciting with a single wavelength and detecting confocally, we chose an approach void of any chromatic aberrations. In any point scanning MINFLUX concept, chromatic aberrations can only play a role in the illumination of the sample, as the coordinate in the sample is fully defined by the illumination beams. Chromatic defects in the confocal detection path can reduce the detected signal, but in contrast to standard camera-based approaches they do not introduce systematic errors in the position estimation. Based on previous publications, we chose dye combinations of Alexa Fluor 647, CF660C and CF680 requiring only minor changes in sample preparation protocols and buffer systems [125]. Using a dichroic mirror, we spectrally split the emitted fluorescence light at around $685 \mathrm{~nm}$ and detected both fractions on single photon counting detectors without making considerable modifications to the main beam paths (see Section 2.1.1). We excited all three dyes at $642 \mathrm{~nm}$ wavelength, but observed a difference in the photon count ratios on the two detectors due to the shift between the emission spectra of the employed dyes (Figure 4.1).

We performed the MINFLUX localization by adding the photons from both spectral channels and treating the resulting photon counts as usual. We classified the emitter 

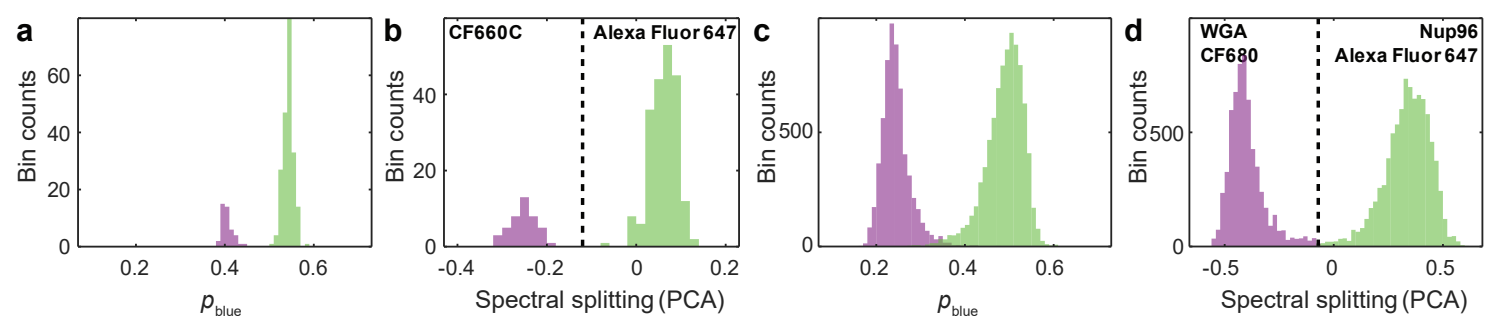

Figure 4.2: Spectral classification in multicolor MINFLUX nanoscopy. a, Distribution of $p_{\text {blue }}$ in last iteration for all localizations shown in Figure 4.3 with Alexa Fluor 647 (green) and CF660C (magenta). Colors were assigned based on the classification in (b). b, Classification of two molecular species for the DNA origami measurement, based on the first principal component of a principal component analysis (PCA), using the photons collected in all iterations. The classification threshold (dashed line) was manually chosen to minimize spectral cross-talk. c, As in (a), but with the distributions for the cellular acquisition in Figure 4.4. The NPC was stained with Alexa Fluor 647 (Nup96, green) and CF680 (wheat germ agglutinin (WGA), magenta). Colors were assigned based on classification (dashed line) in (d). d, Classification of two molecular species based on a PCA as in (b), now for cellular measurement. Material from: Gwosch, K.C.*, Pape, J.K.*, Balzarotti, F.* et al., MINFLUX nanoscopy delivers 3D multicolor nanometer resolution in cells, Nature Methods, 2020, Springer Nature.

species for individual localizations by adding the photon counts from all MINFLUX exposures for each spectral detector. During measurements we classified molecules based on the spectral fraction of photons on the blue-shifted detector $(\lambda \lesssim 685 \mathrm{~nm})$ defined as

$$
p_{\text {blue }}=\frac{n_{\text {blue }}}{n_{\text {blue }}+n_{\text {red }}}
$$

in the last iteration (Figure 4.2a and c). We observed displaced peaks for Alexa Fluor $647\left(p_{\text {blue }} \sim 0.5\right)$, CF660C $\left(p_{\text {blue }} \sim 0.4\right)$ and CF680 $\left(p_{\text {blue }} \sim 0.25\right)$ (Figure 4.2a,c). Cellular environments led to a broadening of the peaks (Figure 4.2c), presumably due to interactions of the dyes with their local chemical environment. To further reduce classification errors, especially for the acquisition of densely labeled cellular structures (see Chapter 5), we exploited the photon counts from all MINFLUX iterations in post-processing. We performed a principal component analysis on $p_{\text {blue }}^{(k)}$ from all iterations $k$, using the probabilities obtained for each individual localization. We then chose a classification threshold based on the distribution of localizations in the first principal component (Figures $4.2 \mathrm{~b}, \mathrm{~d}$ ). This approach delivered negligible spectral cross-talk in most acquisitions.

DNA origami imaging We studied the performance of multicolor MINFLUX based on a custom-made DNA origami structure with Alexa Fluor 647 and CF660C molecules immobilized at distances of 10-12 nm (Figure 4.3a) [19] . Multicolor MINFLUX recovered the position of the labels and correctly classified molecular 

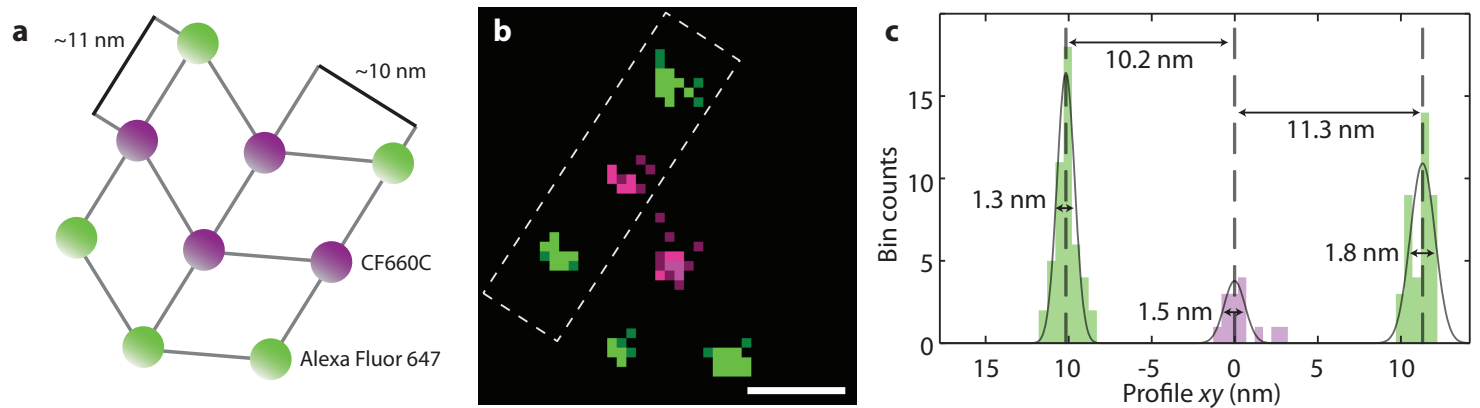

Figure 4.3: 2D two-color MINFLUX image of a custom DNA origami. a, Designed positions of the fluorescent molecules at a distance of $10-12 \mathrm{~nm}$ based on a modified Rothemund rectangular origami. $\mathbf{b}$, MINFLUX image of DNA origami showing the two dye species. c, Histogram of localizations along the area indicated in (b). The average positions have a distance of 10-12 nm. The FWHM for the individual localizations ranges between 1-2 nm. Scale bar: $10 \mathrm{~nm}$ (b). Material from: Gwosch, K.C.*, Pape, J.K.*, Balzarotti, F.* et al., MINFLUX nanoscopy delivers 3D multicolor nanometer resolution in cells, Nature Methods, 2020, Springer Nature.

species (Figure 4.3b). Single localizations showed a full-width at half-maximum of 1-2 $\mathrm{nm}$ corresponding to a localization precision of $\sigma \sim 1 \mathrm{~nm}$. We should note that an offset of the designed distances to the actual positions of the molecules on the order of our localization precision can arise due to varying $\mathrm{MgCl}_{2}$ concentrations, buffer specifications and immobilization strategies [98].

Nuclear pore imaging in 3D We applied 3D MINFLUX combined with the multicolor detection scheme to image the NPC in U-2 OS cells. We used the same cell line as before, so that we could stain Nup96-SNAP using Alexa Fluor 647. Additionally we incubated the cells with wheat germ agglutinin (WGA) conjugated to CF680, known to bind to the center of the NPC [70]. We indeed observed CF680 to reside in the center of the pore laterally as well as axially (Figure 4.4a,c), while Alexa Fluor 647 showed a similar distribution as before. Based on a co-alignment of the average position of all molecules, we obtain a 1D localization precision of $2-4 \mathrm{~nm}$ for both color channels and all dimensions, slightly worse than in the single-color acquisition. Again the precision along the optical axis was slightly better. Also, Alexa Fluor 647 localizations showed slightly better precision than the positions obtained with CF680. To evaluate the distribution of Nup96 and WGA perpendicular to the nuclear envelope, we clustered single NPCs and estimated the shape of the nuclear envelope by spline interpolation (see Section 2.3.4). We subtracted the estimated shape of the nuclear envelope from the localizations. The two Nup96 layers show a distance of about $46 \mathrm{~nm}$, with the WGA staining residing in between (Figure 4.4c).

In conclusion, we could show that MINFLUX allows multicolor 3D imaging in cells with nanometer localization precision and negligible cross-talk in the molecular 

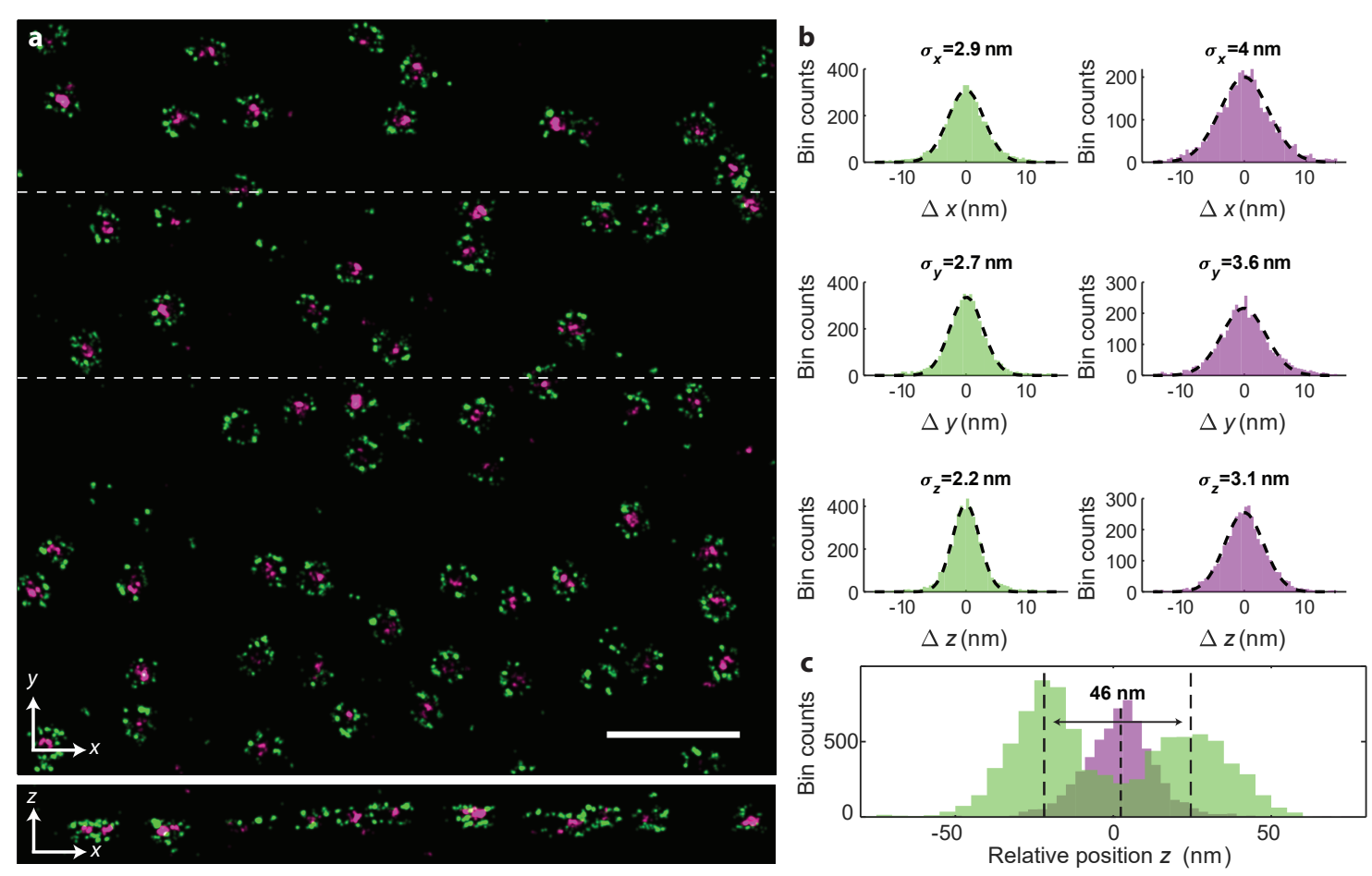

Figure 4.4: Two-color 3D MINFLUX image of Nup96 and center of the NPC in a U-2 OS cell. a, Nup96-SNAP labeled with Alexa Fluor 647 (green) and WGA conjugated to CF680 (magenta), expected to stain the center of the pore. $x y$ - and $x z$-projection of MINFLUX localizations rendered as Gaussian spots, with the color chosen according to the molecule classification. $x z$ slice with projection of the $x y$-region enclosed with dashed lines. $\mathbf{b}, 1 \mathrm{D}$ localization precision for both species after co-aligning the localizations from all molecules. c, Axial average of localizations after subtracting a spline interpolation of the nuclear envelope surface. The two Nup96 layers are estimated to be $46 \mathrm{~nm}$ apart. Scale bar: $500 \mathrm{~nm}$ (a). Material from: Gwosch, K.C.*, Pape, J.K.*, Balzarotti, F.* et al., MINFLUX nanoscopy delivers 3D multicolor nanometer resolution in cells, Nature Methods, 2020, Springer Nature.

classification. Compared to a single-color acquisition (see Section 3.3), we obtained slightly inferior localization precision values. This could be due to an increased background level arising from a higher labeling density. Further, Alexa Fluor 647 delivered a superior localization precision compared to CF680. One possible explanation is the lower signal level of CF680 compared to Alexa Fluor 647 leading to a reduced SBR value. The lower signal level can, among others, be attributed to the lower absorption cross section at the excitation wavelength. Another possible reason is the close proximity of the labels at the center of the pore, so that dye-dye interactions become more likely. If, as a consequence, simultaneous emission of more than one CF680 molecule occurs, the obtained localization represents the average position (weighted by the effective molecular brightnesses) of all emitting molecules. Nevertheless, we demonstrated first multicolor imaging with MINFLUX achieving 
nanometer isotropic localization precision in 3D. This approach is likely to commence a new era of quantitative distance measurements in optical microscopy.

\subsection{Multicolor MINFLUX for tracking applications}

In the previous section we studied multicolor MINFLUX for imaging of static samples. Even if multicolor MINFLUX imaging can allow for the distance measurements of protein species in fixed cells (see Chapter 5) it does not permit to study the dynamics of molecular distances. This is mainly because molecules from both species are registered stochastically and sequentially in time, so that the image acquisition takes minutes to hours. In consequence, the resulting image would be blurred by movements in the sample rather than resolving the underlying dynamics.

If only one fluorescent molecule is present within an enlarged diffraction-limited volume, the switching of the employed molecules is not required for precise localization, so that movements can be followed in time with nanometer precision [26, 123]. To study dynamic molecular distances at the level of single molecules, single-molecule fluorescence resonance energy transfer (FRET) is commonly used, profiting from the distance-dependent interaction of close-by fluorophores [30]. The interaction range of fluorescent dyes is restricted to $2-8 \mathrm{~nm}$ for a dye pair with Förster radius of $5 \mathrm{~nm}$ [92]. This means that FRET does not allow for the measurement of conformational changes or states of large proteins or protein complexes. Several approaches aimed at nanometer-precision co-localization studies of pairs or groups of single emitters within $>10 \mathrm{~nm}$ distance, staying far below the diffraction limit. Most of them did not achieve time-resolved acquisitions.

One group measured the distance between quantum dots or energy-transfer fluorescent beads with spectrally shifted emission at $10 \mathrm{~nm}$ precision. The group used a confocal microscope to avoid errors due to chromatic aberrations [3, 67]. The employed fluorescent markers have not been broadly applied for fluorescent tagging of biomolecules. Other techniques avoided chromatic aberrations by localizing molecules of the same species based on the step-wise photo-bleaching observed in intensity traces $[35,86]$. These approaches used the information about the position of the remaining molecule to infer the other molecule's position, which essentially prohibits time-resolved measurements. A third type of techniques used pairs of dyes with different excitation wavelengths in combination with an extensive color registration scheme to study the molecular distances with nanometer precision [15, 80, 84]. In these studies a significant fraction of the photon budget of the employed dyes was used for a single distance measurement, rendering studies of changes in distance almost impossible. Additionally, camera-based methods do not only suffer from chromatic aberrations when using spectrally shifted dyes, but are also sensitive to the dipole orientation of the involved molecules. This can potentially lead to significant systematic localization errors [27, 69]. 
Here, we studied the applicability of MINFLUX for the simultaneous registration of multiple emitters with shifted emission spectra. By design, the proposed localization scheme is void of any chromatic aberrations and is not influenced by the molecular orientation. Additionally, the improved photon efficiency observed for single-emitterbased MINFLUX, if applicable to the multi-emitter case, can potentially allow for the observation of movements within a range of molecular distances not covered by any other technique. This can, for example, allow to capture the movements involved in protein synthesis or help to unravel the dynamics of intrinsically disordered proteins.

\subsubsection{Theory of multicolor MINFLUX tracking}

We studied the expected performance of the simultaneous registration of spectrally shifted molecules by using the information theoretical approach described in Section 1.2.2. We assumed a group of molecules at positions $\vec{r}_{0}, \cdots, \vec{r}_{M-1}$, omitting any time dependence of the position for legibility. The molecules are illuminated with $K$ spatially and temporally displaced exposures with intensity distributions $I_{i}(\vec{r})$ with $i \in\{0, \ldots, K-1\}$. The photons are collected on $D$ spectrally different detectors. We define a detection probability matrix $\Omega$ describing the probability of photon-detection for each molecule and detector

$$
\Omega=\left(\begin{array}{ccc}
p\left(\operatorname{det}_{0}, \operatorname{mol}_{0}\right) & \cdots & p\left(\operatorname{det}_{D-1}, \mathrm{~mol}_{0}\right) \\
\vdots & \ddots & \vdots \\
p\left(\operatorname{det}_{0}, \operatorname{mol}_{M-1}\right) & \cdots & p\left(\operatorname{det}_{D-1}, \mathrm{~mol}_{M-1}\right)
\end{array}\right)
$$

so that $\sum_{d=0}^{D-1} \Omega_{d m}=1$ holds for all $m \in\{0, \ldots, M-1\}$.

Following Equation 1.19, we obtain the multinomial parameters

$$
\begin{aligned}
p_{i, d}\left(\vec{r}_{0}, \ldots, \vec{r}_{M-1}\right) & =\frac{\sum_{m=0}^{M-1} \lambda_{i, d, m}\left(\vec{r}_{m}\right)+\lambda_{i, d}^{(\mathrm{bg})}}{\sum_{i^{\prime}=0}^{K-1} \sum_{d^{\prime}=0}^{D-1}\left\{\sum_{m=0}^{M-1} \lambda_{i^{\prime}, d^{\prime}, m}\left(\vec{r}_{m}\right)+\lambda_{i^{\prime}, d^{\prime}}^{(\mathrm{bg})}\right\}} \\
& =\frac{\sum_{m=0}^{M-1} b_{m} \Omega_{d m} I_{i}\left(\vec{r}_{m}\right)+\lambda_{i, d}^{(\mathrm{bg})}}{\sum_{i^{\prime}=0}^{K-1} \sum_{d^{\prime}=0}^{D-1}\left\{\sum_{m=0}^{M-1} b_{m} \Omega_{d^{\prime} m} I_{i^{\prime}}\left(\vec{r}_{m}\right)+\lambda_{i^{\prime}, d^{\prime}}^{(\mathrm{bg})}\right\}} \\
& =\frac{1}{\mathrm{SBR}+1}\left[\frac{\mathrm{SBR} \cdot \sum_{m=0}^{M-1} b_{m} \Omega_{d m} I_{i}\left(\vec{r}_{m}\right)}{\sum_{i^{\prime}=0}^{K-1} \sum_{d^{\prime}=0}^{D-1} \sum_{m=0}^{M-1} b_{m} \Omega_{d^{\prime} m} I_{i^{\prime}}\left(\vec{r}_{m}\right)}+\frac{1}{D \cdot K}\right]
\end{aligned}
$$

for the $i$-th exposure and the $d$-th detection channel, assuming the background photons to follow a Poisson distribution with mean $\lambda_{i, d}^{(\mathrm{bg})}$ and an effective molecular brightness $b_{m}$ for each molecule. The last step is equivalent to Equation 1.67, where we don't explicitly write the dependence of the SBR on all molecule positions $\vec{r}_{0}, \ldots, \vec{r}_{M-1}$. We defined the signal-to-background ratio as a combined value for all 
molecules, detectors and exposures

$$
\operatorname{SBR}\left(\vec{r}_{0}, \ldots, \vec{r}_{M-1}\right)=\frac{\sum_{i=0}^{K-1} \sum_{d=0}^{D-1} \sum_{m=0}^{M-1} b_{m} \Omega_{d m} I_{i}\left(\vec{r}_{m}\right)}{\sum_{i=0}^{K-1} \sum_{d=0}^{D-1} \lambda_{i, d}^{(\mathrm{bg})}} .
$$

In Equation 4.5 we further assumed the background contribution to be constant for all exposures and detection channels. We observed this to be a good approximation even for our spectrally different detectors (see Section 4.2.2), where about $40 \%$ of the background photons were detected in the blue-shifted channel. For a situation with low SBR, this assumption should be reconsidered. We represent the effective molecular brightness of molecular species $m$ as $b_{m}$ containing contributions from both the overall detection efficiency and the photo-physical properties of the dye for excitation at a chosen wavelength. We capture all spectral characteristics of emission and detection in the detection probability matrix, so that $b_{m}$ does not depend on the detector index $d$. Notably, the multinomial parameter vector here depends on the relative molecular brightness of the employed dyes, so that it cannot be approximated by ratios of intensities as in the case of a single emitter localization (see Equation 1.19).

We inspected the lower bound on the two-dimensional localization precision in the absence of background. We used two detection channels, two molecules and four exposures arranged in a triangular shape with additional exposure in the center (Figure 4.5a). Based on a quadratic approximation of the excitation intensity distribution, we numerically evaluated the lower bounds on the $4 \mathrm{D}$ localization precision providing analytic expressions for the multinomial parameter vector and its derivatives to cut down computation time. To reduce the parameter space, we kept the molecules fixed at positions $\vec{r}_{1}=[-8 \mathrm{~nm},-8 \mathrm{~nm}], \vec{r}_{2}=[4 \mathrm{~nm}, 4 \mathrm{~nm}]$ from the TCP center. We used a beam separation $L=50 \mathrm{~nm}$, a total number of $N=500$ photons emitted from both molecules and counted on both detectors. Further, we assumed a spectral splitting of $\Omega_{00}=0.5, \Omega_{01}=0.3$ as observed for Alexa Fluor 647 and CF680 respectively (see Section 4.1) and relative molecular brightnesses of $b_{0}=b_{1}=1$. We studied the localization precision for the red-shifted molecule 2 if not indicated otherwise.

Similarly to the single-emitter case, the CRB on the localization precision depended approximately linearly on the beam separation $L$ (Figure $4.5 \mathrm{~b}$ ). We identified the spectral overlap of the dyes as a critical factor for the achievable precision (Figure $4.5 \mathrm{c})$. The lowest error was achieved for full spectral separation of the dyes $\left(\Omega_{00}=1\right.$, $\Omega_{01}=0$ ), reaching $0.76 \mathrm{~nm}$ for 1000 collected photons. Under the same conditions, single-emitter MINFLUX delivered a lower bound on the precision of about $0.5 \mathrm{~nm}$ for an emitter at the TCP center. The numbers cannot be compared directly as (1) in the two-emitter case the photons are split between the two molecules, depending on their exact positions in the TCP and (2) the molecules are not located at the TCP center. For equal photon emission from both molecules located at the TCP 
center, we would expect a precision of $0.5 \mathrm{~nm} \cdot \sqrt{2} \sim 0.7 \mathrm{~nm}$, slightly lower than what we observe. For the spectral separation obtained for Alexa Fluor $647\left(\Omega_{00}=0.5\right)$ and CF680 $\left(\Omega_{01}=0.3\right)$ when splitting the fluorescence light at $\lambda \sim 685 \mathrm{~nm}$ (see Section 4.1 ), the precision was already reduced by a factor of about 3.2 , delivering a 1D error of $2.5 \mathrm{~nm}$ for 1000 collected photons.

We further considered the dependence of the precision on the distance between the molecules by radially modifying the position of the blue-shifted molecule (Figure 4.5d). The precision hardly depended on the distance, but there was a slight tendency towards larger errors for increasing distances. The effect grew with stronger spectral overlap. Two reasons possibly play a role. With the blue-shifted molecule located closer to the TCP center, it absorbs fewer excitation photons. The relative photon collection is thus shifted towards the molecule under observation, leading to an improved 1D error. For the single emitter case, we further know that moving the molecule closer to the TCP center increases the localization precision. The decreasing distance of the second molecule to the TCP (minimal for $d \approx 5.5 \mathrm{~nm}$ ), might also render the photons emitted by the molecule under observation more informative.

We suspected the emission from the second molecule to have a similar effect like background emission. To test this hypothesis, we calculated the dependence of the CRB on the beam separation $L$ for different detection probability matrices (Figure 4.5e). In contrast to a simple background contribution, a second molecule with known emission characteristics in the field of view led to an increased slope of $\sigma_{1 \mathrm{D}}(L)$ with stronger spectral overlap. This means that the gain in photon efficiency when reducing the beam separation $L$ is larger for spectrally similar molecules. The photon efficiency was decreasing for $L<L_{\mathrm{opt}}$, which can be attributed to the positions of the molecules not coinciding with the TCP center. The optimal $L_{\text {opt }}$ showed a slight dependence on the spectral overlap, however, not comparable to the dependence on the background levels (Figure 3.4 for 3D case and [6] Figure S3 for 2D case). This means that the second molecule has an effect different from background on the localization precision of the first molecule. This difference can most likely be attributed to the spatial and spectral dependence of its photon count contribution. The different steepness in photon efficiency indicates that a strong spectral overlap can, up to a certain point, be compensated by clear differences in the photon counts from different multiplex positions. The contrast between the beam target positions becomes larger for a smaller $L$, so that shrinking $L$ compensates more and more for the strong spectral overlap.

We further studied the effect of spectral overlap in more detail by calculating the $\mathrm{CRB}$ for a larger number of detection probability matrices (Figure 4.6a). The 1D error followed curved isolines reflecting an improved precision if the photon counts were not equally split on both detectors or equivalently $\Omega_{00}+\Omega_{01} \neq 1$. When moving away from the line of equal detection probability $\Omega_{00}=\Omega_{01}$ while keeping $\Omega_{00}+\Omega_{01}$ constant (dashed lines in Figure 4.6a), the localization precision diverges with $1 /\left|\Omega_{00}-\Omega_{01}\right|$ for $\left|\Omega_{00}-\Omega_{01}\right| \rightarrow 0$ (Figure 4.6b). This means that two molecules 
a

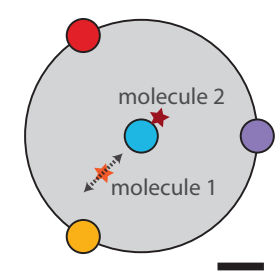

b

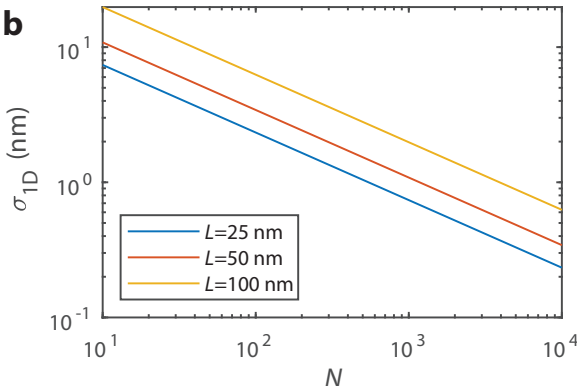

d

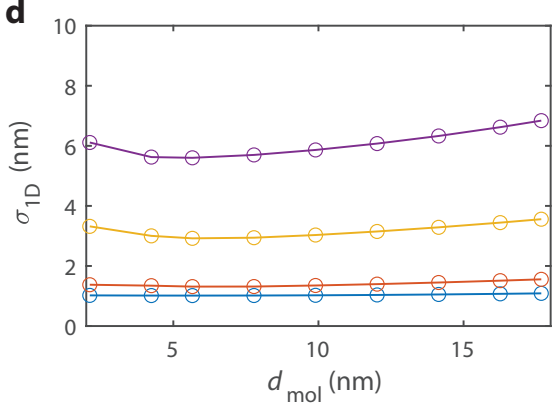

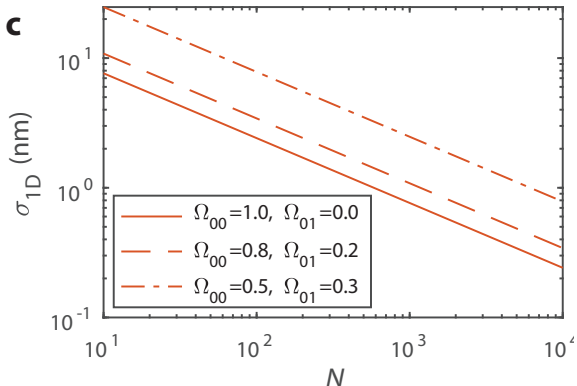

e

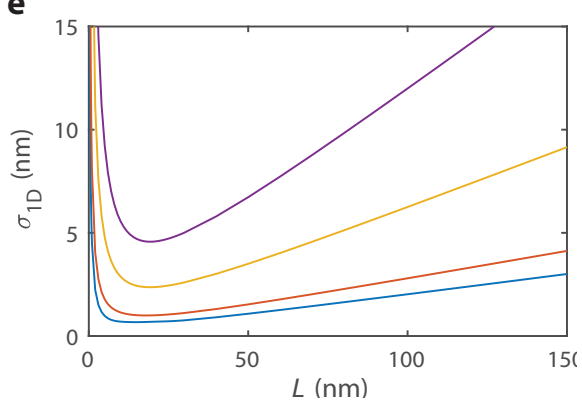

Figure 4.5: CRB on the localization of one molecule when localizing two emitters simultaneously. a, TCP with $L=50 \mathrm{~nm}$ and molecule positions $\vec{r}_{1}=$ $[-8 \mathrm{~nm},-8 \mathrm{~nm}], \vec{r}_{2}=[4 \mathrm{~nm}, 4 \mathrm{~nm}]$ that were used for (b, c, e). For (d) molecule 2 was kept in the same position while molecule 1 was moved radially. $\mathbf{b}$, Dependence of the CRB for the estimation of molecule position 2 (solid lines) on the total number of photons emitted from both molecules for different beam separations $L$ and $\Omega_{00}=0.5, \Omega_{01}=0.3$. Equal molecular brightness $b_{0}=b_{1}=1$ and negligible background contributions were assumed. c, Dependence of the CRB for the estimation of molecule position 2 (red lines) on the total number of photons emitted from both molecules for different detection probability matrices. Same parameters as in (b) and $L=50 \mathrm{~nm}$. d, Dependence of the CRB for the estimation of molecule position 2 (dots and connecting lines) on the distance to the other molecule for different detection probability matrices using $L=50 \mathrm{~nm}$ and $N=500$. e, Dependence of the CRB for the estimation of molecule position 2 on the beam separation $L$ for different detection probability matrices with $N=500$. The same color assignment as in (d) holds. Scale bar: $10 \mathrm{~nm}$ (a).

with identical emission properties cannot be localize confidently. For $\left|\Omega_{00}-\Omega_{01}\right| \rightarrow 1$ the improvement in photon efficiency is not as strong any more. The isotropy shows a similar dependence on the spectral splitting compared to the localization precision (Figure 4.6c). This indicates an overall gain in performance for improved spectral separation of the employed dyes. The isotropy never reaches unity, meaning that there is always a difference in localization precision either between the two molecules or between spatial directions.

We additionally found that using more spectral detection channels helps to improve the CRB. Moreover, using more exposures, in the limit meaning to perform continuous scanning along Lissajou patterns can reduce anisotropies in the localization. 

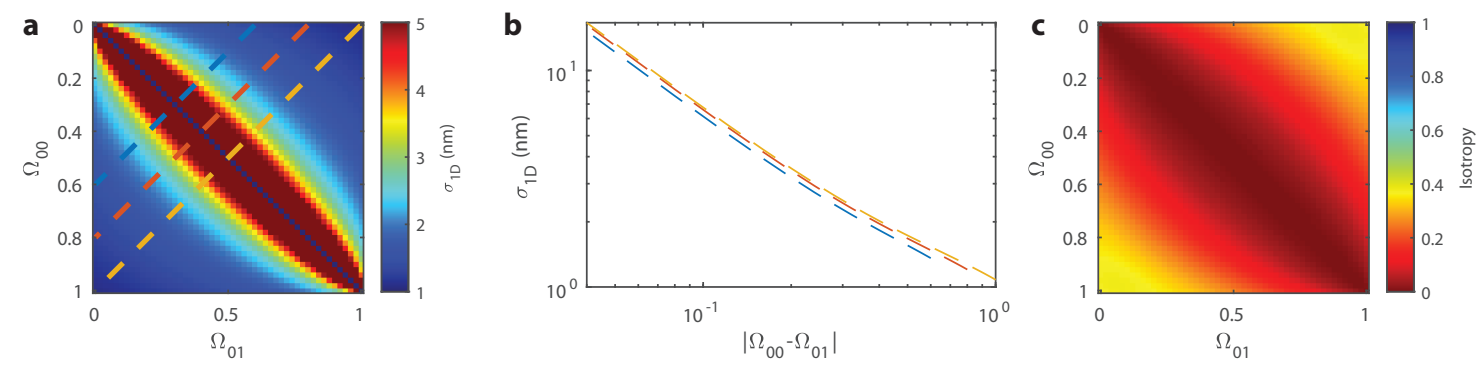

Figure 4.6: Influence of detection probability matrix on CRB and isotropy. We used $N=2000, L=50 \mathrm{~nm}$ and molecule positions $\vec{r}_{1}=[-8 \mathrm{~nm},-8 \mathrm{~nm}], \vec{r}_{2}=[4 \mathrm{~nm}, 4 \mathrm{~nm}]$. We further assumed negligible background contributions. a, CRB for the estimation of molecule position 2 (color-coded) for different spectral detection probabilities with lines of constant sum of detection probability $\Omega_{00}+\Omega_{01}$ (dashed). The corresponding line profiles are shown in (b). b, Line profiles for $\Omega_{00}+\Omega_{01}=$ const. following dashed lines in (a). c, Overall istoropy of the covariance matrix for both molecules using different detection probability matrices $\Omega_{d m}$.

We further studied the performance of the MLE for the simultaneous estimation of 4 position values (two for each molecule). We implemented the MLE in a $4 \mathrm{D}$ gridsearch for $L=50 \mathrm{~nm}$, with a red-shifted molecule at position $[4 \mathrm{~nm}, 4 \mathrm{~nm}]$ in the presence of a blue-shifted molecule at position [ $-8 \mathrm{~nm},-8 \mathrm{~nm}]$ (Figure 4.7). If the molecules did not overlap spectrally, the MLE reached the CRB at about $N=300$ photons collected from both molecules (Figure 4.7a). In comparison, the MLE for a single emitter at the TCP center reaches the CRB at around $N \sim 100$ photons under the same conditions, but using donut-shaped excitation beams instead of a quadratic approximation [6]. For slight spectral overlap $\left(\Omega_{00}=0.8, \Omega_{01}=0.2\right)$, the MLE reached the CRB even for photon numbers below $N=100$, for even larger spectral overlap, the MLE delivers lower standard deviations than the CRB suggested. This directly implies that the MLE delivers a biased estimate of the molecules' positions because no consistent estimator can reach lower errors than the CRB. We studied the consistency of the estimator by calculating the bias on the position estimation (see Equation 1.32). The bias decreased with increasing photon numbers, but did not reach a constant level, indicating that consistency was not fully reached even when localizing with 10000 photons. Using about 10000 photons under experimental conditions (yellow curve), the bias was an order of magnitude lower than the spread of the localizations. Using typical photon count rates of $200 \mathrm{kHz}$, the collection of 10000 photons corresponds to time bins of about $50 \mathrm{~ms}$. To achieve higher temporal resolutions in tracking experiments by using fewer photons per localization, the development of an unbiased estimator would be necessary similarly to the numerically unbiased least mean square estimator as presented in [6]. Also increasing the photon count rate can improve the temporal resolution while keeping the localization precision constant. For MINFLUX to be functional, the mean of the molecular photon emission has to be proportional to the illumination intensity at 

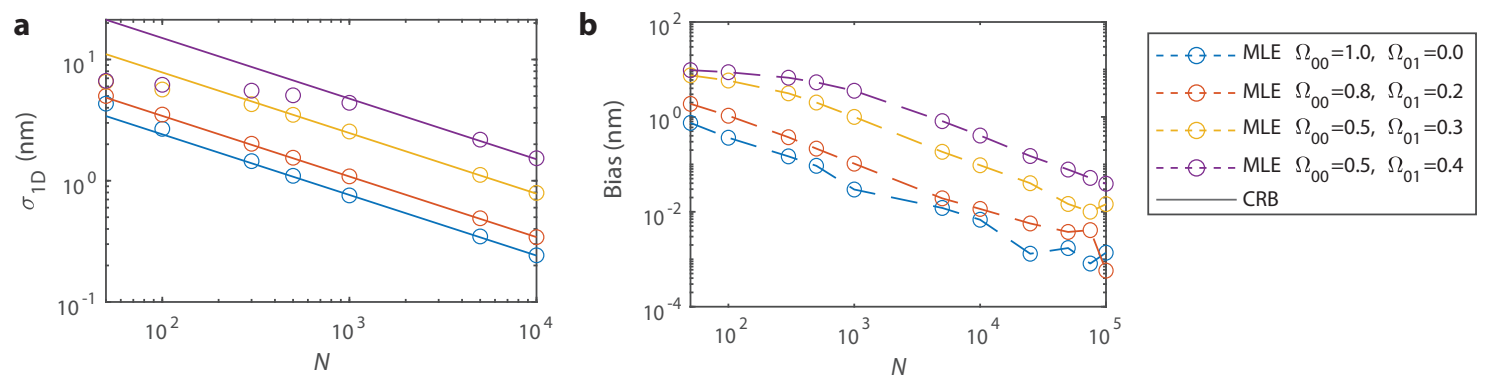

Figure 4.7: Performance of the MLE when localizing two emitters simultaneously. We used $L=50 \mathrm{~nm}$, molecule positions $\vec{r}_{1}=[-8 \mathrm{~nm},-8 \mathrm{~nm}], \vec{r}_{2}=[4 \mathrm{~nm}, 4 \mathrm{~nm}]$ and $b_{0}=b_{1}=1$ for the simulation and omitted background contributions. We used 10000 photon realizations to evaluate the performance of the MLE. a, CRB (lines) and 1D error reached with the MLE (dots) for molecule 2 using different photon numbers $N$. The employed detection probability matrices are indicated in (b). b, Bias of the position estimation (dots and dashed line).

the position of the molecule. This means that saturation of the fluorescence emission has to be strictly avoided.

Overall, the theoretical bounds on the localization precision for two-emitter MINFLUX with potential application in tracking experiments promise major improvement over published camera-based or confocal approaches. When using a single excitation wavelength, the approach is void of chromatic aberrations and polarization-induced errors due to a fixed dipole orientation of the emitters. Further, it potentially allows several hundreds of nanometer-scale localizations from the photon budget of two emitters, allowing for highly improved time-resolved observation of distances. Due to the cross-talk in the spectral detection of the fluorophores we cannot expect the same performance as for single-emitter MINFLUX tracking [26].

\subsubsection{Experimental implementation}

We performed first proof-of-concept measurements on rectangular DNA origami structures with two molecules immobilized in a distance of roughly $24 \mathrm{~nm}$ (see Section 2.4.1). We pre-annealed the rectangular template and added the marker strands later to maintain flexibility in the choice of dyes. For a first experiment we chose the dye combination ATTO 647N and ATTO 665, exciting at $642 \mathrm{~nm}$ and spectrally splitting the fluorescence at around $685 \mathrm{~nm}$, using the microscope described in Section 2.1. We measured the detection probability matrix by acquiring MINFLUX traces of double-stranded DNA labeled with the according dye diluted in folding buffer. We obtained

$$
\Omega=\left(\begin{array}{ll}
0.37 & 0.63 \\
0.25 & 0.75
\end{array}\right)
$$



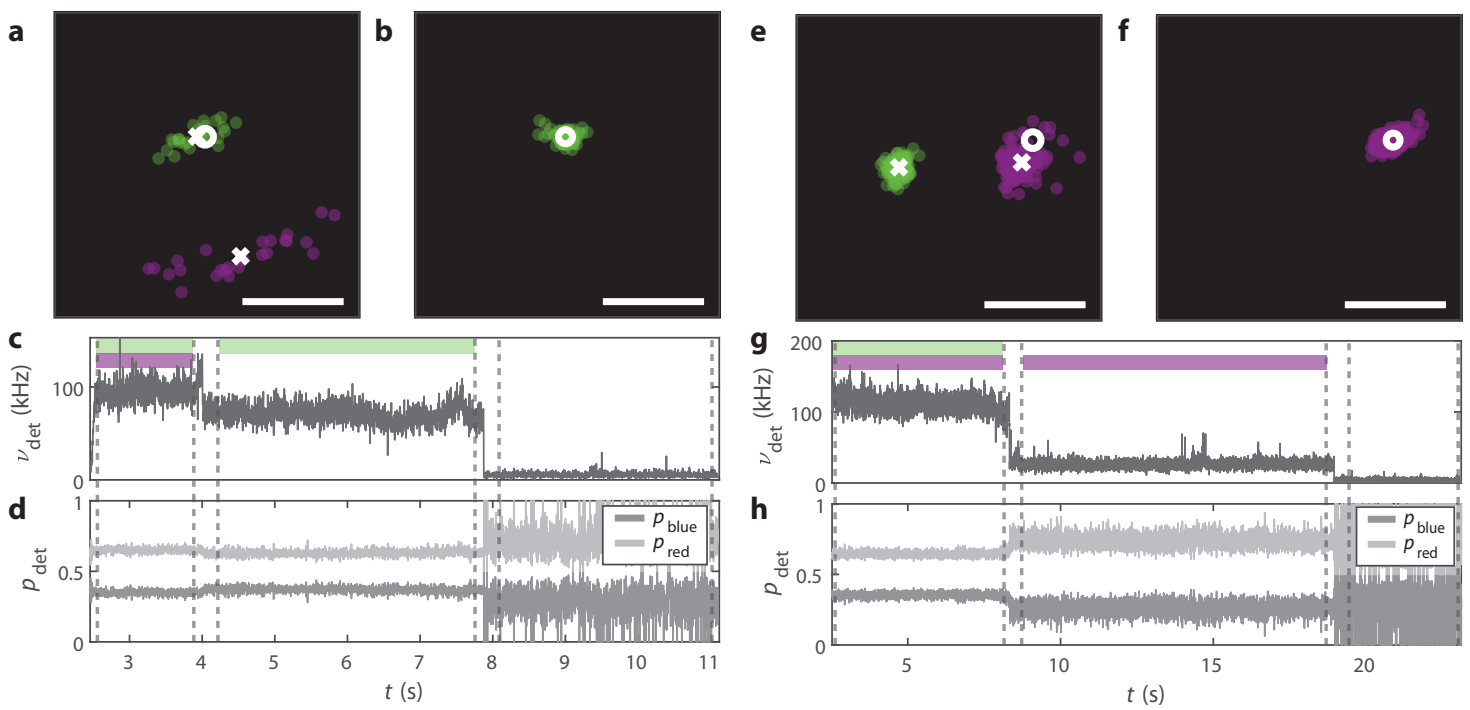

Figure 4.8: Experimental demonstration of simultaneous two-emitter localization. a, e, Position of a single ATTO 647N molecule (green) in a distance of $24 \mathrm{~nm}$ from a simultaneously emitting ATTO 665 molecule (magenta), both immobilized on a DNA origami structure for two different measurements. Single localizations are displayed as semi-transparent scatter points. The size of the points is unrelated to the obtained precision. Mean position for both molecules (white cross) and the position of the emitter remaining after the first photo-bleaching step (white circle). b, f, Individually localized emitter remaining after first bleaching step (green/magenta) with mean position (white circle). c, g, Photon count traces of the molecules shown in (a, b, e, f) for all MINFLUX exposures and detection channels. In the first manually selected trace segment (dashed lines) both molecules emit (green and magenta). After a bleaching step, only one molecule remains in the second segment (green (c) or magenta (g)). The photon counts after the second bleaching step are used for background estimation. $\mathbf{d}, \mathbf{h}$, Spectral detection probability for the photon count trace shown in (c, g). Scale bars: $20 \mathrm{~nm}(\mathrm{a}, \mathrm{b}, \mathrm{e}, \mathrm{f})$.

where we assigned molecule index 0 to ATTO $647 \mathrm{~N}$ and molecule index 1 to ATTO 665. We further measured the brightness of individual emitters by immobilizing biotinylated double-stranded DNA labeled with a single dye molecule on the coverslip. We acquired a confocal image of the single molecules, fit Gaussian distributions to isolated single molecules and used the peak intensity of the fit result as a measure of the molecular brightness. We obtained a brightness ratio of

$$
\frac{b_{\text {ATTO } 665}}{b_{\text {АTTO } 647 \mathrm{~N}}}=0.31 \pm 0.10
$$

where the error is the propagation of the standard deviation of the peak intensities measured for the individual molecules.

We acquired MINFLUX traces of the two-emitter sample in ROXS buffer (see Section 2.4.7) using the static MINFLUX approach with a beam separation of $L=$ 
$70 \mathrm{~nm}$ as described in [26]. To avoid the observation of DNA origami agglomerations, but observe exactly two emitters, we carefully selected traces with two bleaching steps and manually segmented the traces into two-emitter region, single-emitter region and background region (Figure $4.8 \mathrm{c}, \mathrm{g}$ ). With the bleaching steps we usually observed a mild, but visible change in the detection probability for the spectrally separated detectors (Figure $4.8 \mathrm{~d}, \mathrm{~h}$ ). We used a Poisson fit to the histogram of background photons to determine the average background value in each time bin. We subsequently determined the SBR value individually for each photon bin containing molecular emission. For the photons collected within the time span where both emitters fluoresced, we split the photons into bins of 500 MINFLUX multiplex cycles, corresponding to $50 \mathrm{~ms}$ time intervals. In this way we obtained a median of 77 localizations from two emitters containing an average of about $4000 \pm 850$ photons each. With an MLE using a simulated-annealing based optimization (Matlab function simulannealbnd), we obtained the two emitter positions (Figure 4.8a, e). We used the simulated annealing algorithm because it found the optimum of the $4 \mathrm{D}$ problem faster than the gridsearch-based approach used for MINFLUX nanoscopy analysis. For position estimation we used the experimentally obtained PSF that we analyzed as described before [6]. For the fraction of the trace with one emitting molecule, we equivalently estimated the position, now using the $p$-functions as reported in [6] and described in Section 1.2.5 (Figure 4.8b,f).

We determined the distance of the molecules by calculating the difference between the mean positions of all localizations. From all 12 measurements with two bleaching steps, we obtained an average and standard deviation $(26.7 \pm 6.8) \mathrm{nm}$ of all distances. More than half of the measurements contained the true distance value of $24 \mathrm{~nm}$ in their $1 \sigma$ error margin. We obtained an average $1 \mathrm{D}$ error of $(2.5 \pm 1.3) \mathrm{nm}$ for ATTO $647 \mathrm{~N}$ molecules and a slightly worse value of $(4.2 \pm 2.8) \mathrm{nm}$ for all ATTO 665 molecules, where we give the standard deviation of all localization errors. Individual measurements sometimes showed larger errors (Figure 4.8a). For some measurements, we further observed the individual molecule positions to significantly deviate from the position obtained in the simultaneous localization of both molecules.

We could demonstrate that the simultaneous localization of several molecules can allow the observation of distances between two molecules with an error of several nanometers. The development of an improved position estimator can further reduce uncertainties as well as the number of photons that has to be used for each localization. This approach promises reduced bias between the localization of both molecules and the localization of a single molecule. A more thorough treatment of the photophysical parameters influencing the position estimate can further allow to lower systematic errors in the localization. The overall performance can be improved by optimizing the dye combination as well as the wavelength at which the fluorescence is split. An increased number of detection channels or exposures can be used to further enhance the localization precision or isotropy of the localization respectively. 


\subsection{Summary and discussion}

In this chapter we introduced multicolor MINFLUX modalities for nanoscopy (Section 4.1) and tracking (Section 4.2) with nanometer precision.

We demonstrated that the positions of two molecular species can be obtained in a single MINFLUX image acquisition. Based on a single excitation line and detection with two spectral channels, we distinguished classes of molecules by their emission spectra with virtually no spectral overlap. We correctly recovered the designed fluorophore positions and species in a custom-made DNA origami structure. By mapping a two-color staining of the NPC we demonstrated that the proposed multicolor MINFLUX approach is compatible with 3D cellular MINFLUX imaging.

We observed only a slight reduction in localization precision compared to a singlecolor acquisition. Factors limiting the localization precision and image quality are mainly the less beneficial photophysical properties of the red-shifted dye variants CF660C and CF680. On the one hand, these dyes show a less stable off-state compared to Alexa Fluor 647 molecules, so that localizations are lost due to spontaneous activation of molecules outside the MINFLUX acquisition region. On the other hand, less stable off-states lead to a higher background level, thus to lower SBR values, which directly relate to a loss in localization precision. The SBR values for the localization of CF660C and CF680 molecules are further reduced by a lower signal level of these dyes compared to Alexa Fluor 647 when exciting at a wavelength of $642 \mathrm{~nm}$. As the registration of all present molecules happens sequentially in time, multicolor acquisitions require more time due to the increased number of molecules within the FOV. In case of predominance of one species, the second species can be strongly underrepresented by the stochastic nature of the single molecule activation. Development of dyes with more stable off-states or subsequent labeling of different species [60] can help to improve multicolor MINFLUX acquisition schemes. With the improved photon efficiency, MINFLUX lowers the demands on the number of photons needed to achieve molecular resolution. This relaxes the constraint on the photon budget in dye engineering, eventually leading to a new class of dye molecules with lower photon budget, but enhanced switching properties.

We further studied the applicability of MINFLUX for multicolor tracking experiments that require the simultaneous localization of more than one molecule. Based on the calculation of the lower bounds on the localization precision and a first preliminary experiment, we demonstrated that MINFLUX allows for the simultaneous localization of two emitting fluorophores with nanometer precision. In the experiment, we were able to recover the expected distance of the fluorophores of $24 \mathrm{~nm}$ with an error of $6.8 \mathrm{~nm}$ and localized individual emitters with $1 \mathrm{D}$ errors of few nanometers.

In its current implementation, this approach suffers from three main drawbacks. First, the approach relies on the knowledge of photo-physical properties of the 
employed molecules, as we used experimentally determined values for the brightness ratio of the dyes and the detection probability matrix for the localization. Due to changes in the local environment, however, the emission of individual emitters can substantially change, leading to an erroneous position estimation. One possible solution is to obtain the spectral information of one molecule after photo-bleaching of the second and estimating the brightness ratio and the spectral distribution of the other emitter together with the molecules' positions. Due to a higher number of estimated parameters, this would diminish the overall localization performance. Secondly, the MLE showed non-negligible bias for spectrally overlapping molecules, so that the development of an unbiased estimator, for example similarly to the numerically unbiased least mean square estimator [6], would be required for a reliable position estimation, especially when using fewer photons. Finally, the overall performance could be strongly improved by reducing the spectral overlap of the employed dyes. This can be achieved by generating a library of dye combinations with the respective spectral splitting and brightness ratio. The measured brightness ratio as well as the detection probability matrix deviated from expectations based on published photo-physical properties, so that the screening would have to be performed experimentally. In the experiments, it is also possible to access the overall photon budget of single dye molecules under different buffer conditions to increase the photon budget for the generation of long localization traces for both molecules. Another approach to reduce the spectral overlap is to optimize the spectral splitting of the fluorescence in the detection. For this we readily implemented a detection with spectrally tunable filters in a MINFLUX microscope that we built during this work. The same system allows for the excitation with three different wavelengths $(488 \mathrm{~nm}$, $560 \mathrm{~nm}$ and $647 \mathrm{~nm}$ ) thus offering more flexibility in the choice of dyes. In simulations, using more than two detection channels further reduced the localization errors, while more exposures per multiplex cycle improved the isotropy of the localizations. If giving up the benefit of not having to consider chromatic aberrations, the spectral overlap can be reduced by using the spectral difference in the excitation spectra of the molecules by interleaved illumination with light of different wavelengths. The previously mentioned microscope provides all technical prerequisite for this type of experiment. If observing molecules in a smaller distance, energy transfer between the dyes has to be considered for a correct position estimation. With a strong increase in complexity regarding the choice of dyes and data analysis, the presented approach can be extended to observe three or more molecules simultaneously. A straightforward extension to 3D localization should allow absolute distance measurements within three-dimensional arrangements.

Even without optimized experimental conditions with respect to the choice of dyes, the spectral detection and the buffer conditions and a non-optimal estimator, multiemitter MINFLUX resolved a distance of $24 \mathrm{~nm}$ in about 80 localizations per molecule pair. The approach thus opens completely new possibilities for the observation of dynamics in protein complexes or other biological conformations. 


\section{MITOCHONDRIAL IMAGING}

In the previous chapters, I presented the development of a MINFLUX localization scheme for 3D and multicolor imaging. The scheme allowed mapping single molecules of more than one species with molecular resolution in all dimensions. So far, the technique has been demonstrated by imaging sparse biological structures close to the cell membrane of eucaryotic cells. In this chapter, I set forth multicolor 3D MINFLUX imaging of a complex organelle sub-structure, namely the mitochondrial crista junction. I further present the development of a data analysis framework based on unsupervised machine learning algorithms to exploit the molecular resolution of MINFLUX for quantitative image analysis. Overall, this chapter demonstrates the applicability of MINFLUX to tackle complex biological questions quantitatively, but also shows, how the improved precision increases the requirements for strict photophysical control of the employed dyes as well as for the labeling approach. I start with a brief introduction on mitochondrial architecture and function (Section 5.1), followed by a proof-of-concept demonstration of two-color 3D MINFLUX imaging in mitochondria with $\sim 5 \mathrm{~nm}$ localization precision (Section 5.2). I describe the development and characterization of an analysis framework for quantitative analysis of MINFLUX data on the molecular level based on simulated data (Section 5.3). Finally, I apply the developed tools for biological imaging and data analysis to unravel information about the protein distribution within the heterooligomeric MICOS protein complex that is located at the crista junctions (Section 5.4). I isolated the distribution of Mic60, the core component of this complex, at single crista junctions from a 3D MINFLUX image of a U-2 OS cell, observing pores of roughly $40-50 \mathrm{~nm}$ size, again with an isotropic localization precision of $\sim 5 \mathrm{~nm}$. Moreover, I used two-color 3D MINFLUX to analyze the spatial relation between different subunits of the MICOS complex. I measured the difference in distance from Mic60 to Mic10 and Mic19, respectively, and found that Mic10 resides significantly farther away from Mic60 compared to Mic19. Thereby, MINFLUX provided remarkable new insights into the spatial organization of MICOS subunits at crista junctions. 


\subsection{Introduction to mitochondrial imaging}

Mitochondria, often called the powerhouses of the cells, are organelles with crucial importance for the energy provisioning in complex organisms. They efficiently convert the energy provided in form of glucose or fat into adenosine triphosphate (ATP) and generate 15 times more ATP from a single glucose molecule compared to anaerobic glycolysis. Mitochondria thus played a major role in the evolution of complex organisms. Moreover, mitochondrial dysfunction or mutations of mitochondrial proteins have been related to numerous diseases $[110,126]$.

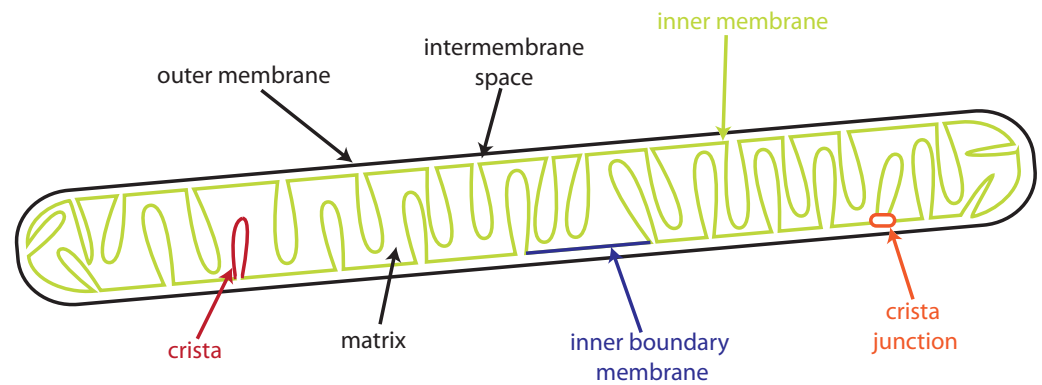

Figure 5.1: Simplified picture of the mitochondrial architecture. The mitochondrion is bounded by two membrane layers, the outer and inner mitochondrial membrane. The inner mitochondrial membrane is usually divided into three sub-domains: crista membrane, inner boundary membrane and crista junctions, which mark the transition between inner boundary and crista membrane.

Mitochondria are bounded by two lipid bilayers, called outer and inner membrane (Figure 5.1). The outer membrane shapes the organelle and is responsible for communication with the rest of the cell as well as for protein transport. The inner membrane contains, among others, the enzymes of the respiratory chain, which preserve an ionic gradient between the matrix and the intermembrane space. The respiratory chain complexes include $\mathrm{F}_{0} \mathrm{~F}_{1}$-ATP-synthase responsible for the conversion of adenosine diphosphate (ADP) to the energy-rich ATP. The inner membrane can be subdivided into three domains: the inner boundary membrane, the cristae and the connecting crista junctions. The inner boundary membrane runs parallel to the outer membrane while the cristae are invaginations into the mitochondrial matrix that increase the surface area of the inner membrane (Figure 5.1). The crista junctions connect inner boundary membranes and crista membranes and are thought to play a key role in compartmentalizing the inner mitochondrial membrane and controlling the transport of proteins and other biomolecules. Crista junctions were for the first time observed and morphologically described based on electron tomography [83], later four studies independently identified a large heterooligomeric protein complex located at the crista junctions $[2,45,54,116]$, now called mitochondrial contact site and cristae organizing system (MICOS) complex [85]. A depletion of MICOS leads to a loss of crista junctions and a disturbed inner membrane structure, underlining 
its importance for the architecture of the organelle. Numerous publications have studied the detailed role of the MICOS complex for mitochondrial structure and function as recently reviewed in [66] and [87]. Functions include the bending of the inner membrane for cristae formation as well as the interaction of outer and inner membrane [87]. Most of the studies have been carried out based on either electron microscopy data or biochemical assays. These studies demonstrated, that the MICOS complex consists of two sub-complexes named after their core-subunits, respectively. In humans, the Mic60-subcomplex includes Mic60, Mic25 and Mic19 whereas a Mic10 sub-complex includes Mic10, Mic27, Mic26 and Mic13 [31, 39, 116]. The spatial organization of the two MICOS sub-complexes remained unknown, however, since conventional fluorescence microscopy could not address this question due to its limited resolution. In recent years, super-resolution microscopy has allowed to image the distribution of numerous mitochondrial proteins $[22,56,57,64]$ and demonstrated a coordinated distribution of Mic60 clusters along yeast and human mitochondria [105].

As the resolution of the employed techniques was still limited to tens of nanometers, imaging single crista junctions and their protein composition remained elusive. Here we developed two-color 3D MINFLUX imaging of mitochondrial proteins as a tool to quantitatively study the arrangement of different components of the MICOS complex at the level of single crista junctions.

\subsection{D MINFLUX imaging of mitochondrial proteins}

One of the most prevalent strategies to label proteins for fluorescence microscopy uses an indirect staining of the target structure. An unlabeled primary antibody targets the protein of interest while a secondary antibody that targets the primary antibody is decorated with fluorescent molecules. Indirect immunostaining approaches offer (1) a lot of flexibility in the choice of dyes and (2) often deliver a bright staining because numerous fluorescent molecules label a single target. The signal amplification happens because several secondary antibodies target a single primary antibody and because one secondary antibody usually carries a number of fluorescent molecules. For quantitative microscopy, indirect immunostaining suffers from three main drawbacks, however. On the one hand, the number of fluorescent molecules per target protein is unknown, shadowing information on the stoichiometry of the structure of interest. On the other hand the fluorescence labels can be located as far as $\sim 30 \mathrm{~nm}$ away from the target structure and can be distributed over a volume of roughly $15 \mathrm{~nm}$ in extend, corresponding to the linear size of a single immunoglobulin G ( $\operatorname{Ig} G$ ) antibody molecule [29]. Moreover, the size of the antibody can additionally lead to steric hindrance, which effectively reduces the labeling efficiency. Numerous alternative labeling strategies as comprehensively summarized in [29] or [95] allow for monovalent binding and small distances between target and labeling molecule. 


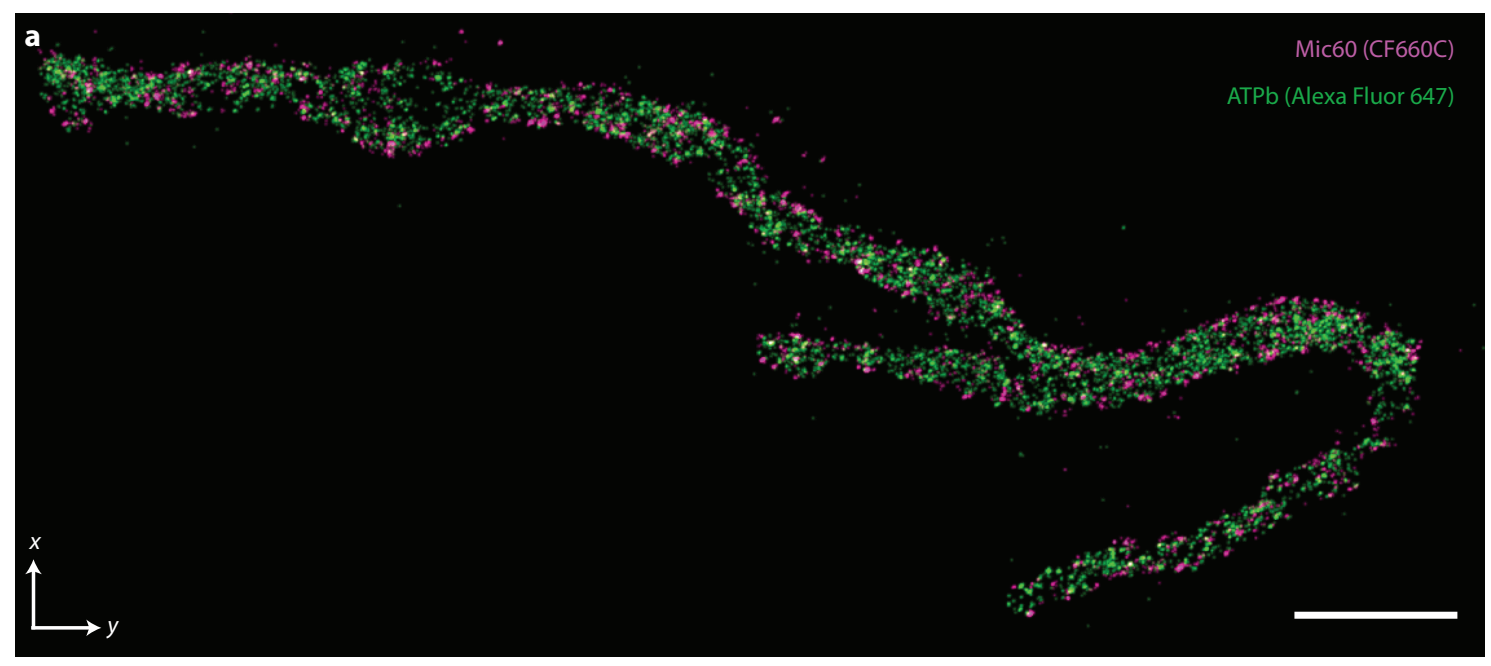

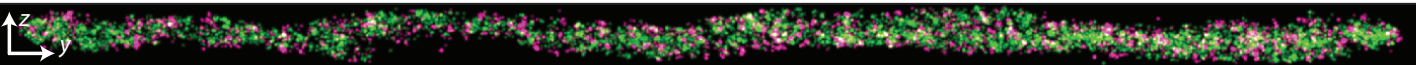
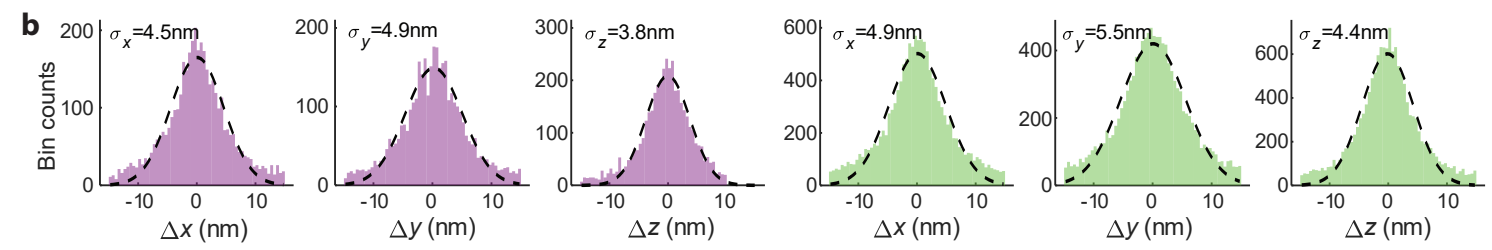

Figure 5.2: Two-color 3D MINFLUX acquisition of mitochondrial proteins in a human dermal fibroblast. a, $x y$ - and $y z$-projection of localizations rendered as $2 \mathrm{D}$ Gaussian distributions with $\sigma=5 \mathrm{~nm}$. Magenta: Mic60, green: $\beta$-subunit of the $\mathrm{F}_{0} \mathrm{~F}_{1}$-ATPase $(\mathrm{ATPb}) . \mathbf{b}$, Localization precision for both species (colors as in (a)) estimated from a 1D Gaussian fit (dashed line) to aligned localizations (histograms). Scale bar: 1 um (a). Material from $[81]$.

Some of the labeling approaches cannot be established easily and with high binding affinity for all proteins of choice (Fab fragments and nanobodies). Others require endogenous labeling thus eventually hampering the protein function in the living cell (SNAP-tag, Halo-tag or peptide tags). For this work, we chose a compromise of binding specificity, simplicity in the sample preparation process and size of the label by labeling primary antibodies directly with the organic dyes used for MINFLUX microscopy (see Section 2.4.6). In this way, the distance of the label to the target structure was reduced and the stoichiometry of the labeling improved compared to an indirect immunostaining protocol, while established protocols could be used for the sample preparation. In theory, up to 8 amino acid residues per antibody can carry a fluorescent molecule. By using MINFLUX imaging of isolated antibodies we found that most of the antibodies were only decorated with one or two fluorescent molecules, however.

For MINFLUX imaging of mitochondrial proteins we first selected a mitochondrion of interest in a widefield fluorescence image. We chose MINFLUX scan points in 
the mitochondrial region, usually not following a rectangular pattern, but a custom shape to avoid increased acquisition times.

We quantified the performance of multicolor 3D MINFLUX imaging inside mitochondria by staining different domains of the inner mitochondrial membrane. In human dermal fibroblasts, we labeled Mic60, located at crista junctions, and the $\beta$-subunit of $\mathrm{F}_{0} \mathrm{~F}_{1}$-ATPase, located at crista membranes (Figure 5.2a). Due to it's more controllable switching behavior, we chose Alexa Fluor 647 to label the abundant ATPase and CF660C for Mic60, respectively. As expected, we observed that the $\mathrm{F}_{0} \mathrm{~F}_{1}$-ATPase molecules resided closer to the mitochondrial axis in average while Mic60 was arranged on a variable cylindrical structure. We resolved the protein distributions within the inner mitochondrial membrane with a $3 \mathrm{D}$ isotropic localization precision of roughly $5 \mathrm{~nm}$ for both species (Figure $5.2 \mathrm{~b}$ ). We could thus demonstrate the first successful MINFLUX acquisition in any organelle. Due to the photon-efficient localization, MINFLUX further delivered images of mitochondrial proteins with unprecedented isotropic 3D resolution.

Due to the pointilistic nature of the MINFLUX acquisition, especially prominent for sparse structures or low labeling efficiencies in combination with high localization precision, only a reduced amount of information can be assessed by visual inspection of a 3D dataset. In the next section we present a framework that we developed for quantitative molecular analysis of MINFLUX data showing mitochondrial proteins.

\subsection{Quantitative molecular analysis of MINFLUX data}

The outcome of any localization-based super-resolution measurement is a coordinate list of localizations usually following Gaussian distributions around the position of each fluorescent molecule. Solving the inverse problem, meaning to assign each localization to a molecule to infer information about molecular clusters, distances and distributions with high fidelity as recently reviewed in [79] remains challenging. This is especially true if localization precision and molecular distance have a similar magnitude. Most developments demonstrated clustering in two dimensions only, because an anisotropic localization precision, as present in most conventional camerabased localization techniques, poses an additional challenge.

By achieving higher localization precision, MINFLUX can substantially push the labeling density range accessible for quantitative microscopy. Here, we developed a clustering algorithm for molecular identification of localizations from any 2D or 3D MINFLUX measurement (step 2 in Figure 5.3). We optimized and verified the molecular identification by simulating localizations for a simplified mitochondrial model. Based on this model, we studied and optimized our biological analysis approach. To this end, we examined the nearest-neighbor distance distributions 


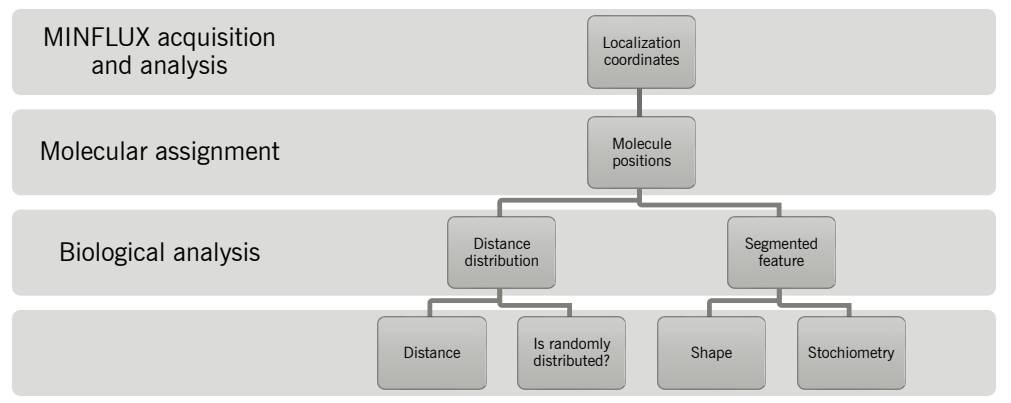

Figure 5.3: Analysis pipeline for biological MINFLUX acquisitions. A MINFLUX experiment with basic analysis delivers localization coordinates distributed around positions of molecules. Localizations are assigned to individual molecules by means of a cluster analysis, so that subsequent quantitative processing steps can be performed on a molecular basis. Biological features can be studied by the distance distribution of molecules or by segmenting features of interest, so that molecular distances, shapes and ideally also protein stoichiometries can be deduced.

a

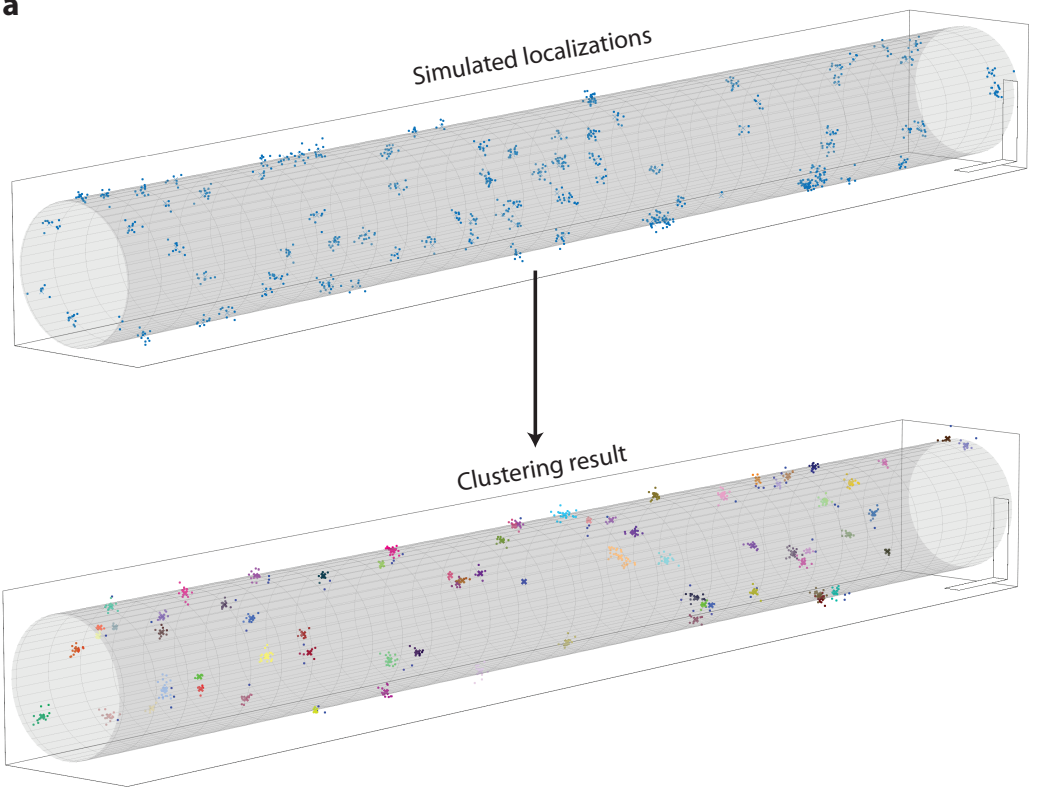

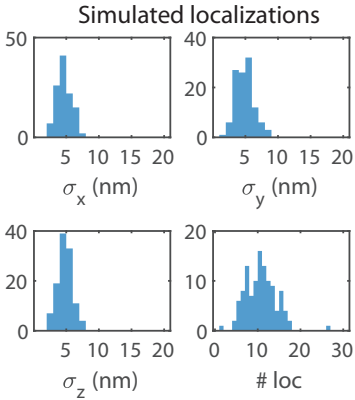
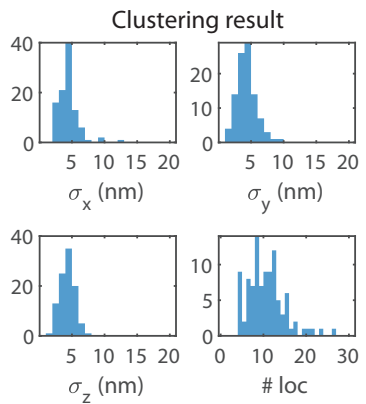

Figure 5.4: Simulation of mitochondrial localization data. a, Simulated localization clouds of roughly 100 molecules at a density of $\rho=100$ molecules $/ \mathrm{um}^{2}$. Molecules were randomly distributed on a cylinder with diameter $D=160 \mathrm{~nm}$ with a mean of 10 localizations per molecule, based on a Poisson distribution. A localization precision of $\sigma=5 \mathrm{~nm}$ was assumed. A clustering with eps $=[10 \mathrm{~nm}, 6 \mathrm{~nm}], \operatorname{minPts}=[3,8]$ and $\sigma_{\mathrm{gmm}}=5 \mathrm{~nm}$ delivered molecular assignments (one color for each assigned molecule). $\mathbf{b}$, The distributions of standard deviations and number of localizations for the ground truth (top) and the clustering result (bottom). Scale bar: $100 \mathrm{~nm}$ (a). 
for two spatially associated molecular species and estimated their average distance (step 3 and 4 in Figure 5.3). We further applied the clustering algorithm with optimized parameters for segmenting features from the simulated data allowing to study shape and stoichiometry of molecular arrangements (step 3 and 4 in Figure 5.3). In Section 5.4, we apply all presented data analysis tools to study the distribution of proteins within single crista junctions.

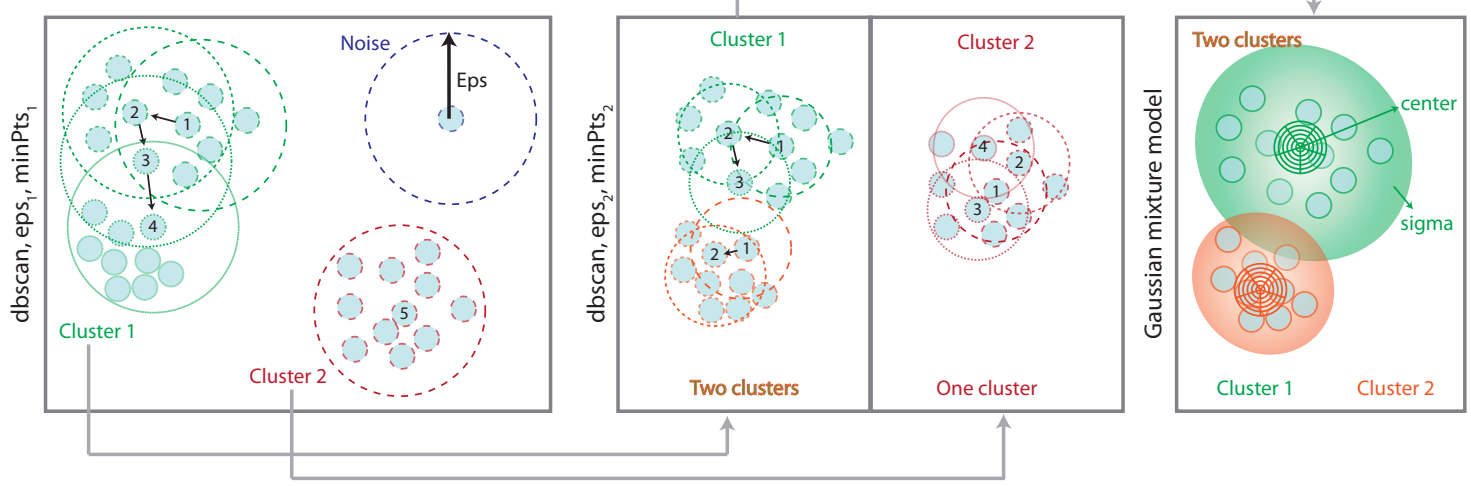

Figure 5.5: 3D clustering algorithm dbscan2 for molecular identification of localizations. The assignment of localizations to molecules is based on a three-step process. In a first step a density-based clustering algorithm $d b s c a n$ assigns all points within a sphere of radius eps to the same cluster, whenever the sphere contains more than minPts localizations (green and red). If a region around a point contains less than minPts localizations and this point is not a boarder point of a cluster, it is referred to as noise (blue). In a second step, a second dbscan with reduced eps finds clusters that are composed of two or more localization-dense regions (green and orange). Within each of these regions, subclusters are found based on a Gaussian mixture model (green and orange) with the mean determined from the second $d b s c a n$ clustering step and $\sigma_{\text {gmm }}$ given as an input parameter. Each localization is assigned to the Gaussian component with highest probability at the localization coordinate. Material from [81].

Simulation of localization data For the simulation of localizations in a mitochondrial model, we assumed a straight tube of diameter $D$ and length $L$. We defined a density $\rho$ in units of molecules per surface area and distributed the molecule positions randomly on the cylinder. We generated localizations around each of the molecule positions assuming an isotropic Gaussian localization distribution with spread $\sigma$ in all three dimensions. We chose the number of localizations per molecule based on a Poisson distribution with mean 10. An example of a generated dataset is shown in Figure 5.4a. To simulate a second molecular species for distance studies, we randomly placed the second molecule on a circle of distance $d$ from the first molecular species. To introduce an uncertainty in the simulated distance we assumed $d$ to follow a Gaussian distribution with spread $\sigma_{d}$. To simulate a model of the crista junction, we 
distributed junctions rather than molecules randomly on the mitochondrial cylinder. For each junction we simulated a predefined number of molecules uniformly arranged on a circle with radius $r$ oriented with the mitochondrial surface. To avoid overlapping junctions, we regenerated positions closer than $3 r$ together.

Molecular identification of localizations For molecular identification of localizations we studied the performance of well-known unsupervised machine learning algorithms. Most of the algorithms (e.g. kmeans) require the number of molecules in the image as an input, a variable usually not known in advance. A suitable candidate among established clustering algorithms is the density-based approach dbscan [28]. In brief, the algorithm distinguishes point-dense regions from empty regions by counting the number of points in the vicinity of radius eps of a single starting point. If the number exceeds a predefined threshold minPts, all points are assigned to the same cluster. If the number does not pass the threshold and none of the points belongs to another cluster, the points are referred to as noise. The process is repeated until all points are assigned to a cluster or defined as noise (see left panel Figure 5.5).

We found that a simple $d b s c a n$ algorithm allowed the identification of molecules fairly well, but no parameter set $\{e p s$, minPts $\}$ allowed to separate close-by molecules while not splitting localizations of single emitters. For this reason, we developed a three-step process as depicted in Figure 5.6. Following a standard dbscan clustering step (left panel), we separated the clusters and applied a second dbscan with modified parameters to each cluster (central panel). Using the mean position of the subclusters identified in the second step and a predefined spread $\sigma_{\mathrm{gmm}}$, we re-assigned individual points to the subcluster with highest probability at the point coordinate based on a Gaussian mixture-model (right panel). We applied the clustering approach
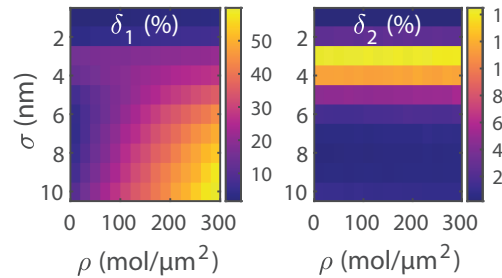
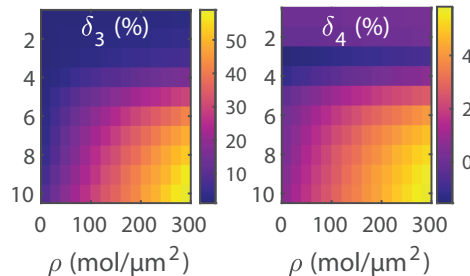
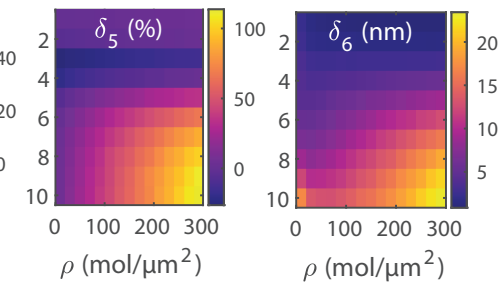

Figure 5.6: Measurement of the clustering error. Errors $\delta_{1}-\delta_{6}$ (color-coded) were obtained from a simulation using a mean number of 10 localizations with precision $\sigma$ from molecules of density $\rho$ on a cylinder with diameter $D=400 \mathrm{~nm}$. The length of the cylinder was adjusted, so that the simulated region contained roughly 500 molecules. The mean clustering error for 100 trials is given. Detailed error definitions, all comparing the clustering result to the ground truth are introduced in the text. For clustering eps $=[20 \mathrm{~nm}, 4 \mathrm{~nm}]$, minPts $=[4,3]$ and $\sigma_{\mathrm{gmm}}=5 \mathrm{~nm}$ were used.

to a simulated dataset and obtained clusters mainly corresponding to individual molecules (Figure 5.4a). The clustering roughly recovered the distribution of standard deviations and number of localizations per molecule (Figure 5.4b). We took a more 
quantitative approach to evaluate the clustering error for different molecular densities $\rho$ and localization precisions $\sigma$ (Figure 5.6). As not only whole molecules, but also single localizations can be wrongly classified, the error of the molecular assignment can be defined in numerous ways. We tested six error metrics, based on either the number of clusters and molecules, or on the nearest-neighbor distances. All metrics compared the ground truth with the clustering result. Using the number of molecules in the model $n_{\text {mol }}$, the number of classified molecules $n_{\text {clus }}$ and the number of identified cluster per ground-truth molecule $n_{\text {mol/clus }}$, we defined

$$
\begin{aligned}
\delta_{1} & =p\left(n_{\mathrm{mol} / \mathrm{clus}} \neq 1\right) \\
\delta_{2} & =p\left(n_{\mathrm{mol} / \mathrm{clus}}<1\right) \\
\delta_{3} & =p\left(n_{\mathrm{mol} / \mathrm{clus}}>1\right) \\
\delta_{4} & =\frac{n_{\mathrm{mol}}-n_{\mathrm{clus}}}{n_{\mathrm{mol}}},
\end{aligned}
$$

where $\delta_{1}=\delta_{2}+\delta_{3}$ holds. We further defined the error metrics

$$
\begin{aligned}
& \delta_{5}=\frac{\bar{d}_{\mathrm{nn}, \text { clus }}}{\bar{d}_{\mathrm{nn}, \mathrm{mol}}}-1 \\
& \delta_{6}=\bar{d}_{\mathrm{nn}, \mathrm{mol} \rightarrow \mathrm{clus}} .
\end{aligned}
$$

based on the average nearest-neighbor distance of ground truth molecules $\bar{d}_{\mathrm{nn} \text {,mol }}$ and assigned clusters $\bar{d}_{\text {nn,clus }}$, respectively, and the average distance of a ground-truth molecule to the nearest assigned cluster $\bar{d}_{\text {nn,mol } \rightarrow \text { clus }}$. We repeated the simulation 100 times for each combination of localization precision and molecular density to reduce the uncertainty on the clustering error estimate. All error metrics except $\delta_{2}$, describing the probability of assigning true localizations to noise, increased continuously with density and scaled inversely with the localization precision (Figure 5.6). Interestingly, this tendency broke, especially visible for $\delta_{2}$, at a localization precision slightly below the clustering radius eps $=4 \mathrm{~nm}$ of the second clustering step. In this case the number of molecules was underestimated. Error metrics $\delta_{1}$ and $\delta_{3}-\delta_{6}$, show very similar shape, but different amplitudes. For further analysis, we chose error metric $\delta_{4}$ because it (1) differentiated between over-clustering and under-clustering by its sign, (2) is dimensionless.

Based on error metric $\delta_{4}$, we evaluated the dependency of the clustering error on the clustering parameters. We found the clustering to be robust towards parameter modification.

By studying the clustering errors and their dependence on the localization density as well as on the clustering parameters, we could confirm, that the presented clustering approach can indeed be used for molecular identification of localizations. As the 
clustering quality did not strongly depend on the clustering parameters, we chose the parameters empirically rather than using a numerical optimization. It is important to note that for a localization precision of $\sim 5 \mathrm{~nm}$ demonstrated for MINFLUX measurements of mitochondrial proteins (Figure 5.2), $\delta_{4} \lesssim 20 \%$ holds even for very high molecular densities of 200 molecules $/ \mu^{2}$. The analysis of the clustering error further demonstrated the power of increased localization precision for quantitative microscopy. Many biological structures, if fully labeled, contain dense protein arrangements that are impossible to resolve and quantitatively study with a standard camera-based precision.
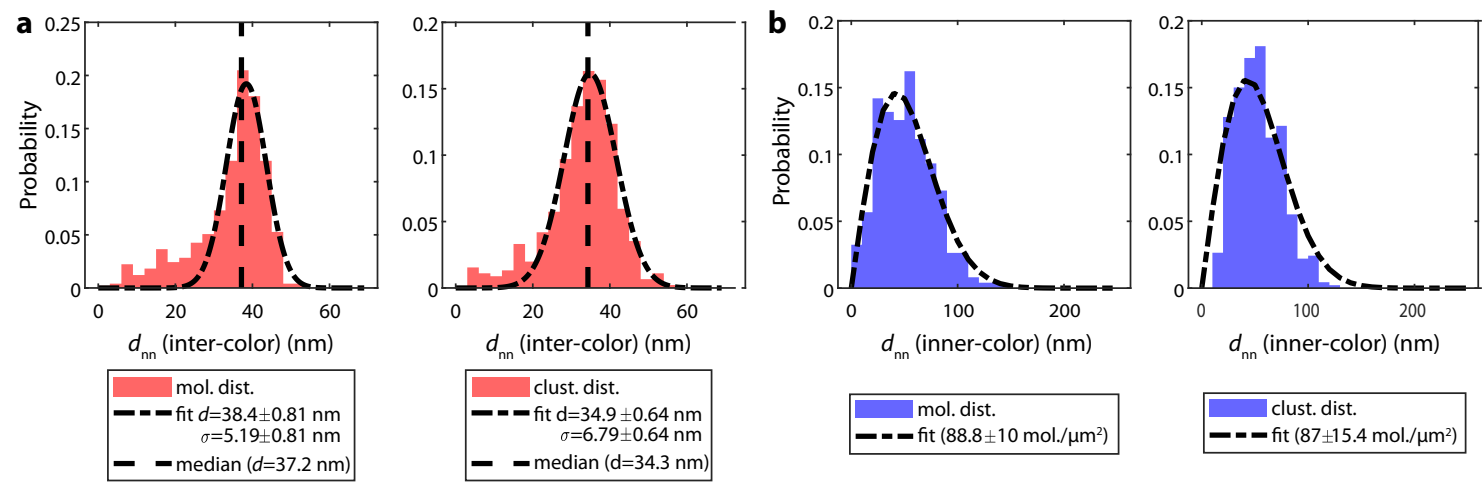

Figure 5.7: Nearest-neighbor distance distributions of two molecular species in simulations. Both species were assumed to be present at a density of $\rho=100$ molecules $/ \mathrm{um}^{2}$ on a cylinder of diameter $D=160 \mathrm{~nm}$. The length of the cylinder was adjusted to obtain roughly 500 molecules of each species. A Poisson-distributed number of localizations with mean 10 and a spatial spread $\sigma=5 \mathrm{~nm}$ were used for both species. The second species was positioned in a Gaussian-distributed distance $d=40 \mathrm{~nm}$ with $\sigma_{d}=4 \mathrm{~nm}$. a, Distribution of inter-species nearest-neighbor distances (histogram) with Gaussian fit (dashed and dotted line) and median value (dashed line) for ground truth (left) and clustering result (right). The error indicates the $95 \%$ confidence interval. The molecular identification used the parameters eps $=[10 \mathrm{~nm}, 6 \mathrm{~nm}]$, minPts $=[3,8]$ and $\sigma_{\mathrm{gmm}}=5 \mathrm{~nm}$. $\mathbf{b}$, Distribution of nearest neighbors within the species. The distribution is expected to follow $p\left(d_{\mathrm{nn}}\right)=2 a \pi \rho d_{\mathrm{nn}} \mathrm{e}^{-\pi \rho d_{\mathrm{nn}}^{2}}$ with molecular density $\rho$ and scaling constant $a$ (fit, dashed and dotted line). The fit result for the parameter $\rho$ is given in the legend for ground-truth molecule distribution (left) as well as for the distribution of identified clusters (right).

Studying molecular distances based on clustered data Based on the molecular identification of localizations, a quantitative analysis of species distances could be carried out. For this, we studied the distribution of nearest-neighbor distances between two species, where the individual inter-species distances were simulated to follow a Gaussian distribution. The resulting distribution of inter-species nearestneighbor distances consequently followed a Gaussian distribution with an additional tail towards lower distances (Figure 5.7a). This was true for both simulated molecules and clustering result. We estimated the mean distance based on a Gaussian fit to the 
distribution. Using the ground-truth molecule positions delivered a distance value of $d_{\mathrm{mol}}=(38.40 \pm 0.81) \mathrm{nm}$ with the error describing the $95 \%$ confidence interval, thus slightly underestimating the true distance $d=40 \mathrm{~nm}$. When using the identified molecule positions, this effect was even stronger, estimating a molecule distance of $d_{\text {clus }}=(34.90 \pm 0.64) \mathrm{nm}$. Using the median value of distances, we obtained an even worse estimate of the distances for ground-truth molecules and clusters. The standard deviation of simulated distances $\sigma=4 \mathrm{~nm}$ was slightly overestimated by the ground-truth-based Gaussian fit $\left(\sigma_{\text {mol }}=(5.19 \pm 0.81) \mathrm{nm}\right)$ as well as when using the clustered data $\left(\sigma_{\text {clus }}=(6.79 \pm 0.64) \mathrm{nm}\right)$. We suspected that a population of molecules with a random nearest neighbor closer than the associated nearest neighbor led to the described systematic deviations. To study this in more detail, we evaluated the inner-species nearest-neighbor distance distribution accounting for randomly distributed molecules. As deduced in [77], the distribution of nearest-neighbor distances $d_{\mathrm{nn}}$ for molecules of density $\rho$ randomly distributed on a surface follows

$$
p\left(d_{\mathrm{nn}}\right)=2 a \pi \rho d_{\mathrm{nn}} \mathrm{e}^{-\pi \rho d_{\mathrm{nn}}^{2}},
$$

with $a$ being a constant. We estimated the density $\rho$ of the molecules by fitting the given probability density function, resulting in density values slightly below the expected. We further observed a tightening of the probability density function for the clustered data compared to the molecular data. The latter is most likely due to the clustering algorithm not separating close-by molecules.

In conclusion, we can estimate the distance between spatially associated species based on a Gaussian fit to the inter-species nearest-neighbor distance distribution. The estimate is biased towards lower values due to a population of random distances that are smaller than the associated distance. This means, that we cannot reliably deduce the absolute distance values using this approach, a comparison of different distance values, however, is possible.

Feature segmentation for isolation of crista junction models The molecular identification of localizations can additionally be used to study the molecular arrangement of proteins within the cell. To extract cellular features, we adapted the parameters of the dbscan2 algorithm displayed in Figure 5.5 and used it for molecular identification of localizations. We avoided the propagation of errors in the molecular assignment by applying the algorithm directly to the localization data, not to the identified molecule positions. We tested the approach by simulating protein arrangements around the crista junction, assuming 6 molecules to be uniformly distributed on a circle with radius $15 \mathrm{~nm}$ (Figure 5.8a). We indeed successfully identified single junctions (Figure 5.8b). To visualize individual junctions that were aligned with the curved surface of the mitochondria (Figure 5.8c), we rotated the junctions by calculating the diagonal matrix of eigenvalues from the covariance matrix of isolated localizations. After rotation, the axis of lowest variance 


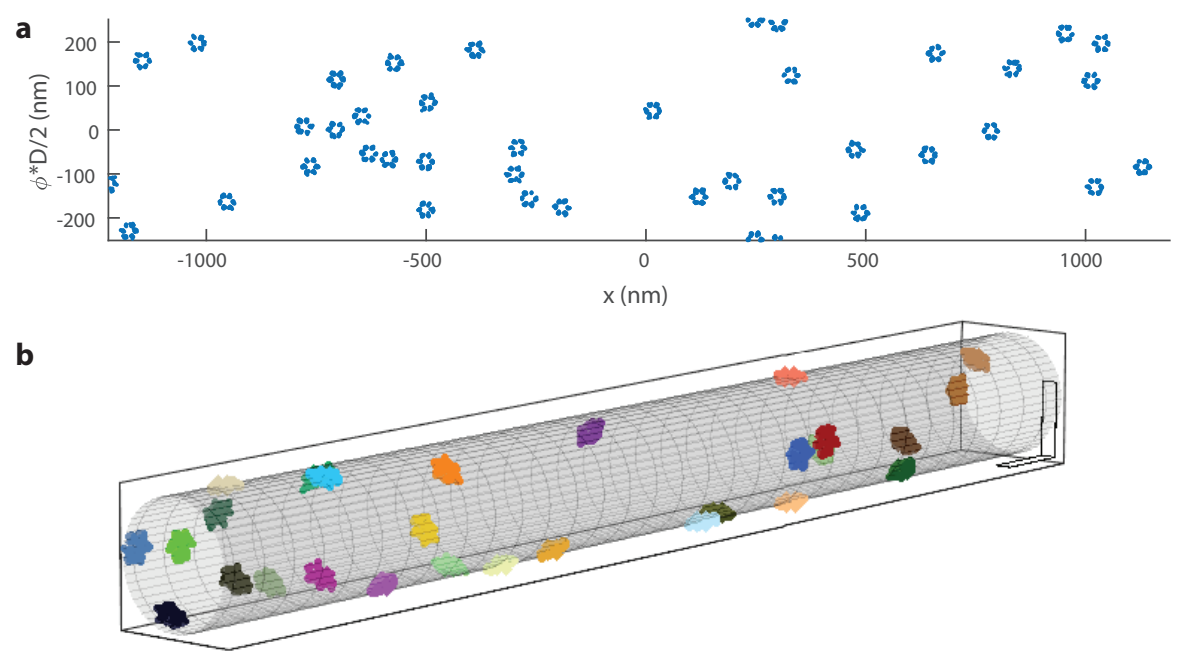

c
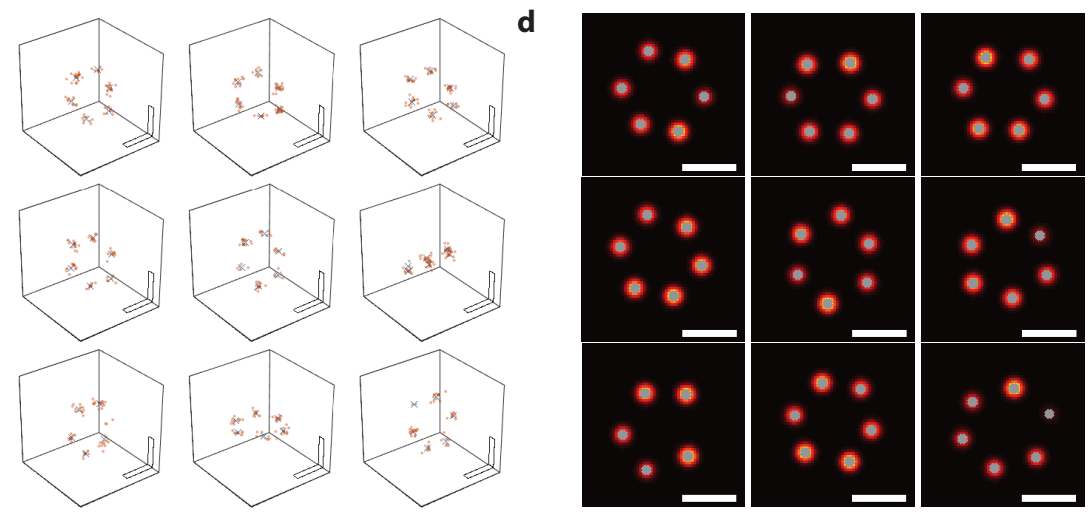

Figure 5.8: Simulation of localizations for a MICOS protein in the crista junction. We assumed a localization precision of $\sigma=2 \mathrm{~nm}$, a mean number of 10 localizations per molecule and a labeling efficiency of $100 \%$. We assumed six molecules per junctions equally distributed on a circle with radius $15 \mathrm{~nm}$. a, Unwrapped cylinder $(D=160 \mathrm{~nm})$ containing localizations of roughly 80 junctions at a density of 30 junctions $/ \mathrm{um}^{2}$. $\mathbf{b}$, Isolated single junctions (one color per junction) and molecule positions within junctions by applying the previously described three-step dbscan2 clustering to the simulated localizations using eps $s_{\text {loc }}=[10 \mathrm{~nm}, 6 \mathrm{~nm}]$, $\operatorname{minPts}_{\mathrm{loc}}=[3,4], \sigma_{\mathrm{gmm}, \mathrm{loc}}=5 \mathrm{~nm}$, eps $_{\text {junction }}=[25 \mathrm{~nm}, 15 \mathrm{~nm}]$, minPts $_{\text {junction }}=[3,10]$ and $\sigma_{\text {gmm,junction }}=20 \mathrm{~nm}$. c, Isolated junctions oriented with the cylinder surface. d, Isolated junctions rotated to the $x y$-plane by using the eigenvectors of the covariance matrix of the localizations. Localizations are rendered as Gaussian distributions with $\sigma=2 \mathrm{~nm}$, identified molecule positions are marked (gray). Scale bars: $100 \mathrm{~nm}(\mathrm{~b}), 20 \mathrm{~nm}(\mathrm{c}, \mathrm{d})$. 
coincided with the projection direction (Figure 5.8d). We also observed successful molecular assignment (Figure 5.8d). For comparison, we performed an analogous evaluation with increased localization errors and $50 \%$ labeling efficiency (Figure A.6).

In summary, we developed a simulation framework for the generation of mitochondrial localization data to optimize and validate new data analysis approaches. For data analysis, we first assigned localizations to molecules based on a combination of unsupervised machine learning algorithms that we termed dbscan2. We showed that the quality of the assignment strongly depends on localization precision and molecular density. We obtained errors in the recovered molecule number of $\lesssim 20 \%$ for experimental localization precisions and densities. The molecular identification recovered the distance between molecular species with slightly more than $10 \%$ error. Further a dbscan2 approach with adapted parameters allowed isolating molecular features that we were able to align for visualization and further analysis.

\subsection{D MINFLUX can resolve protein distributions within the crista junction}

In the last section, we presented a simulation and data analysis approach to quantitatively analyze MINFLUX acquisitions of mitochondrial proteins. Here, we apply the developed techniques to study the protein distribution within single crista junctions. We isolated the distribution of Mic60 from single junctions (Section 5.4.1). We further compared the distance between two protein pairs within the MICOS protein complex (Section 5.4.2).

\subsubsection{Mic60 distribution at single crista junctions}

To study the distribution of Mic60 within single crista junctions, we acquired singlecolor 3D images of Mic60 in U-2 OS cells using a primary antibody labeled with Alexa Fluor 647 (Figure 5.9a). Already by visual inspection we observed larger arrangements that presumably do not originate from single molecules. To analyze the shape of the arrangements, we applied the developed feature segmentation approach and again rotated the localizations and identified molecule positions to study the isolated junctions (Figure 5.9b). As the feature segmentation recognized also a number of single molecules as junctions, we manually selected a subset of the identified junctions. We observed that the Mic60 proteins tended to be arranged in regions of roughly $40-50 \mathrm{~nm}$ with individual molecules separated by roughly $20 \mathrm{~nm}$. Note that the alignment of the arrangement with the $y$ axis is caused by the rotation based on the covariance matrix eigenvalues.

We, for the first time, observed indications for Mic60 arrangements within single crista junctions in U-2 OS cells with optical microscopy. Our data suggest that 
the crista junctions, which have a diameter of about $20 \mathrm{~nm}$ on electron micrographs (unpublished data), are surrounded by a group of Mic60 molecules, delivering a diameter of $40-50 \mathrm{~nm}$ in our measurements using a staining with antibodies. As Mic60 is essential for the formation of crista junctions and bends lipid vesicles into tubular structures in vitro $[53,106]$, it is attempting to assume, that these groups of Mic60 surround and thereby form the crista junctions. To draw more quantitative conclusions regarding the shape and stoichiometry of the identified pores, higher labeling efficiencies and a statistical analysis of a larger dataset would be required. We should note that 3D resolution is strictly necessary for the observation of these arrangements as a projection can cause artificial pores that do not correspond to single junctions.
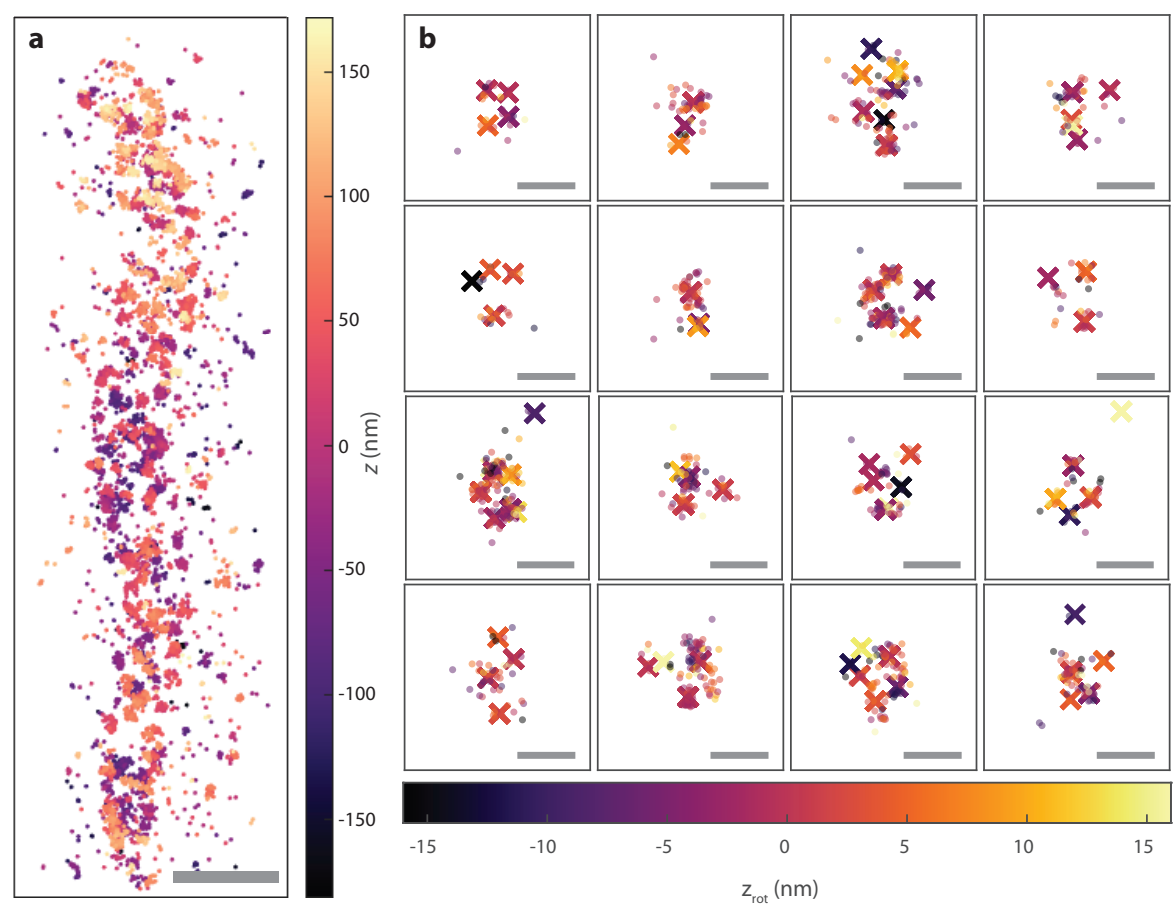

Figure 5.9: Single-color 3D MINFLUX acquisition of Mic60 in a U-2 OS cell. a, MINFLUX localizations with the axial position color-coded. Mic60 was labeled with Alexa Fluor 647. b, Isolated crista junctions (subplots) with localizations (dots) and assigned molecules (crosses) aligned based on the covariance matrix of the localizations. The third dimension after rotation $z_{\text {rot }}$ is color-coded for all datapoints. Scale bars: $500 \mathrm{~nm}$ (a), $50 \mathrm{~nm}$ (b). Material from [81].

\subsubsection{Distances of subunits within the MICOS complex}

We studied the difference of protein distances within the MICOS complex using two-color MINFLUX nanoscopy. Again, we required the isotropic 3D resolution of 

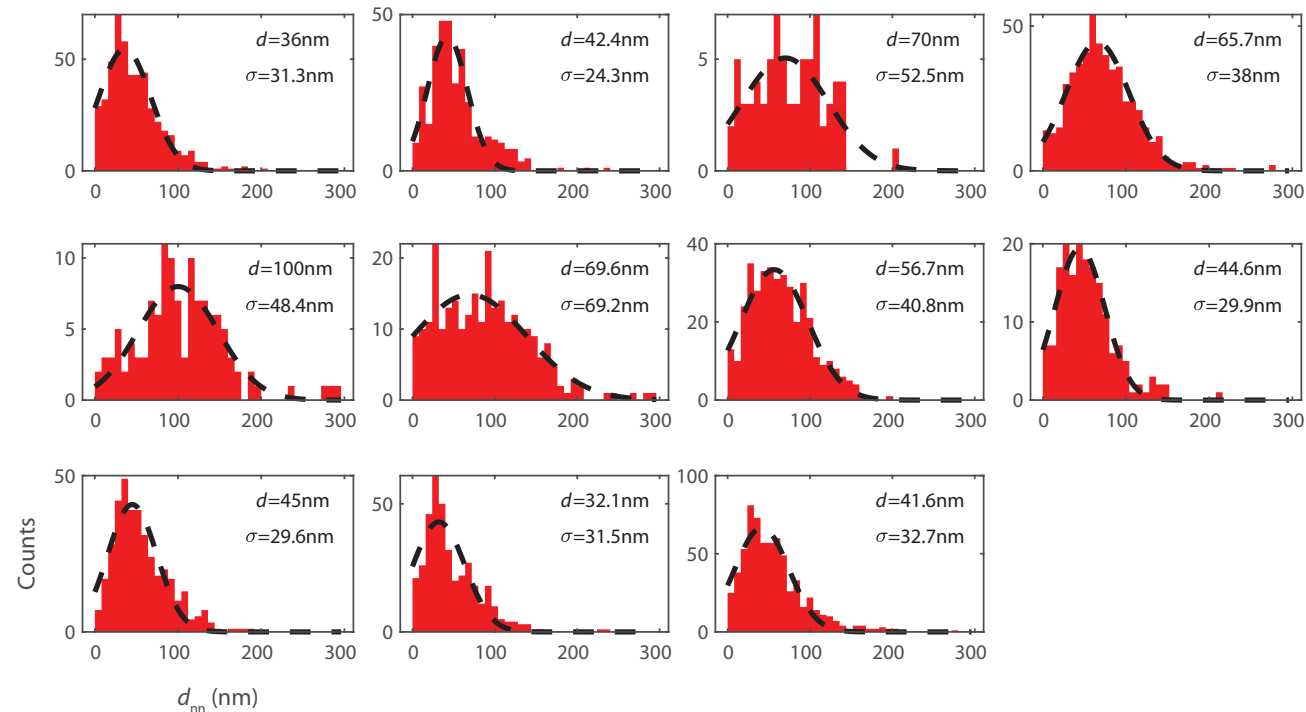

b

Nearest neighbor distance distribution Mic60-Mic19
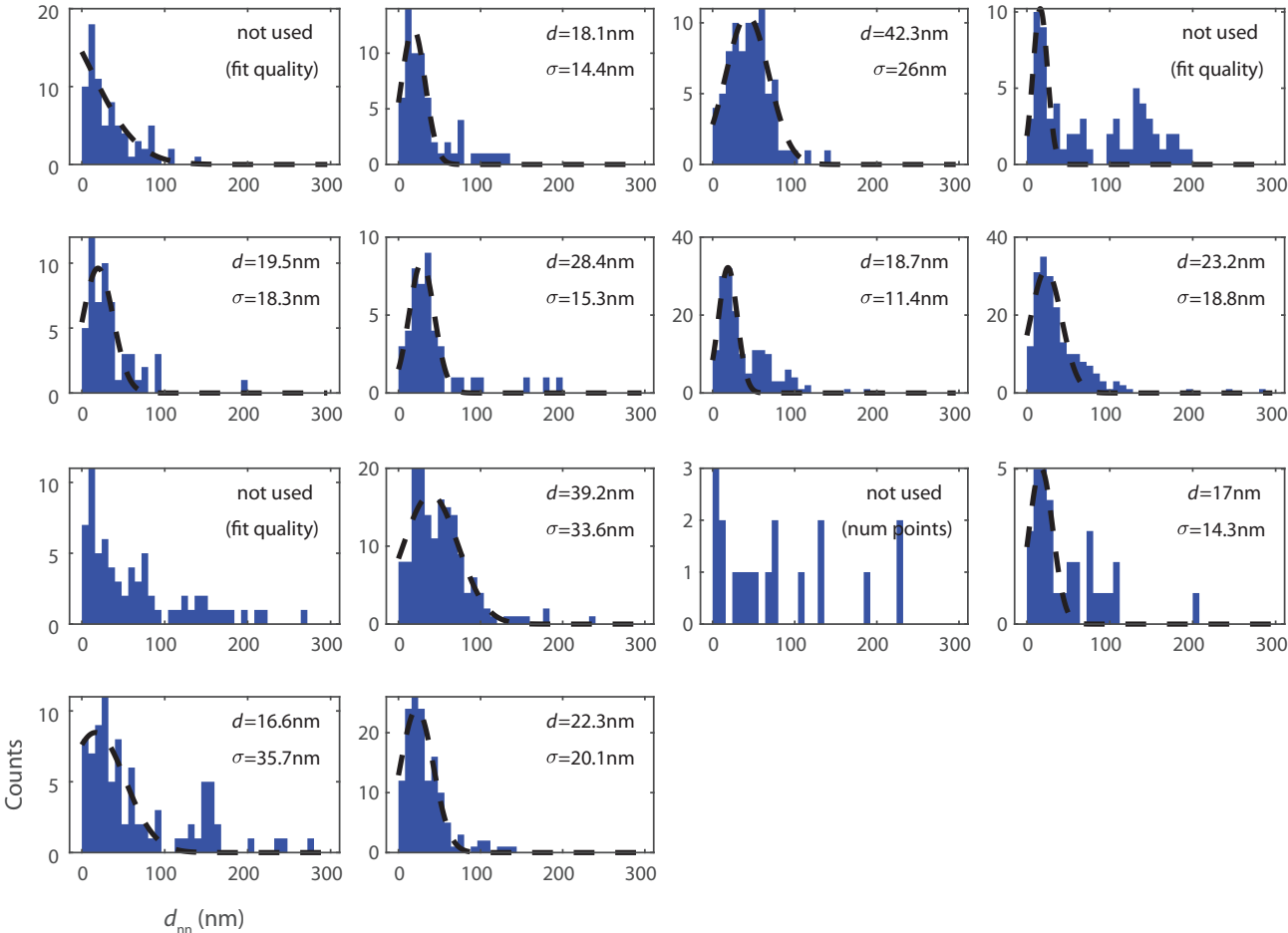

$d_{\mathrm{nn}}(\mathrm{nm})$
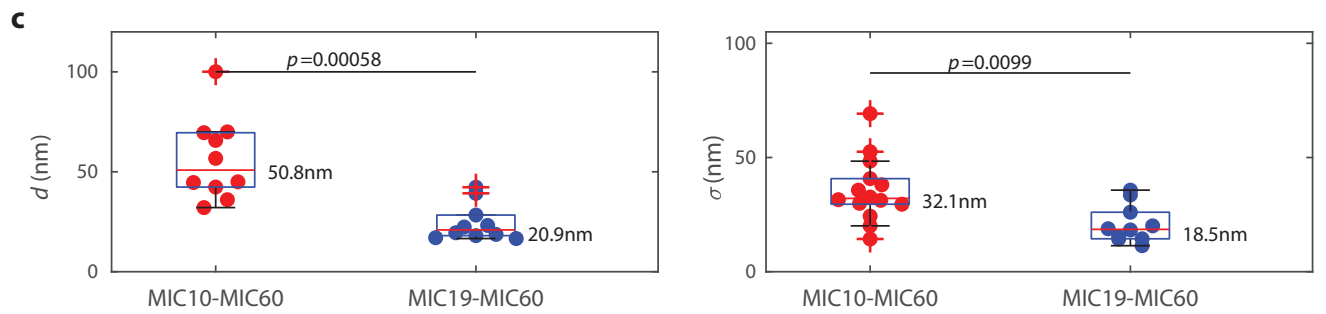
Figure 5.10: Distances of MICOS proteins based on a molecular identification of localizations. For all distance measurements, the less abundant species was identified and the nearest-neighbor distance to the other species calculated. a, Distribution of nearest-neighbor distances between Mic10 and Mic60 for acquisitions from 11 cells on 5 coverslips. A Gaussian fit (dashed line) delivers the mean distance and the spread of distances as indicated. Measurements with less than 30 molecules in the less abundant species (not used, num points) or either coefficient of determination $R^{2}<0.5$ or negative mean values (not used, fit quality) were excluded from further analysis. b, Same as (a), but measuring the distance between Mic19 and Mic60. c, Average distance (left) and distance spread (right) for all measurements. The data points show individual measurements. The plot indicates the median value of the distribution (red line and numbers), the $25 \%$ and $75 \%$ percentiles (blue box) and the extreme values (error bar). Outliers are marked with a red cross. The hypothesis that the distances and spreads for Mic10 and Mic19 belong to the same distribution was tested based on a whitneyU test (Matlab function ranksum). The resulting $p$-value is given for both tested parameters. Material from [81].

MINFLUX, as distance measurements based on projected positions of molecules that natively reside on a tube are highly error-prone. We imaged HeLa cells, where we labeled Mic60 as a reference along with Mic10 and Mic19, respectively. We observed that Mic19 occurred at a lower molecular density than Mic10, which could be due to a lower abundance of the Mic19 protein, but also due to a lower binding affinity of the employed antibody. For all datasets we used the same filtering values as in Figure 5.2 (see Section 2.3.3 and Figure A.4).

To compare the distances between Mic10 and Mic60 to the distance of Mic19 to Mic60, we identified molecules based on the approach described in Section 5.3. We selected the species of lower abundance and found the nearest neighbors in the other species for all molecules to obtain a distance distribution for all acquired datasets (see Figure 5.10a, b). The resulting inter-color nearest-neighbor distance distributions roughly followed a Gaussian distribution. We used a Gaussian fit to identify the expected molecular distance and the standard deviation of molecular distances in each measurement (Figure 5.10a, b). We excluded datasets with less than 30 molecules in the less abundant species and measurements with low fit quality (coefficient of determination $R^{2}<0.5$ or negative mean value) from further analysis as indicated.

We compared the resulting distributions of expected distances and $\sigma$ values (Figure 5.10c). We obtained a median distance of $\sim 50 \mathrm{~nm}$ for Mic10-Mic60 and a lower median distance of $\sim 20 \mathrm{~nm}$ for Mic19-Mic60 without considering the size of the antibody used for labeling. A WhitneyU hypothesis test delivered a probability of only $p=0.00058$ for both distance measurements to originate from the same distribution, indicating that the distances of Mic60 to Mic10 and Mic19 respectively were significantly different. Also the median value of $\sigma$ for all distance distributions was larger for the distance Mic60-Mic10 compared to Mic60-Mic19. 
In conclusion, we were able to measure a difference in distance of three proteins that are known as subunits of one large heterooligomeric complex. We observed Mic10 and Mic60 to be significantly farther apart than Mic19 and Mic60. This result is consistent with biochemistry data showing that Mic19 belongs to the same MICOS subcomplex as Mic60 whereas Mic10 is part of a different subcomplex [31, 39, 116].

\subsection{Summary and discussion}

In this chapter we demonstrated for the first time multicolor 3D MINFLUX imaging of an organelle in mammalian cells. We showed first proof-of-concept measurements in human dermal fibroblasts where we labeled Mic60 and $\mathrm{F}_{0} \mathrm{~F}_{1}$-ATPase. For labeling we used primary antibodies decorated with fluorophores suitable for multicolor MINFLUX imaging. We achieved an isotropic localization precision of $\sim 5 \mathrm{~nm}$. We presented a data analysis framework, developed based on the simulation of localizations. The framework allowed to assign localizations to individual fluorescent molecules, based on a combination of unsupervised machine learning algorithms that we termed dbscan2. The molecular assignment enabled further analysis using the molecule positions rather than the individual localizations. We used the obtained molecule positions to establish a distance measurement based on the distribution of nearest-neighbor molecular distances. An adapted version of the density-based clustering used for molecular identification allowed the isolation of molecular arrangements from the simulated data.

We applied the analysis framework to study the distribution of Mic60 in U-2 OS cells based on 3D MINFLUX imaging data. We isolated regions that resembled crista junctions, and observed pores of $\sim 40-50 \mathrm{~nm}$ in size. Other publications already suggested that Mic60 forms oligomers [53] and that Mic60 can bend lipid vesicles into tubular structures in vitro $[53,106]$. Further, crista junctions in U-2 OS cells showed a diameter of roughly $20 \mathrm{~nm}$ in electron micrographs (unpublished data). Taking this information together, it is thus tempting to assume that the groups of Mic60 molecules observed in the MINFLUX data, indeed surround and thereby form single crista junctions.

Using two-color 3D MINFLUX imaging of HeLa cells, we compared the molecular distance of Mic60 to Mic10 and Mic19, respectively. We obtained a sample median of $\sim 50 \mathrm{~nm}$ for the distance Mic10-Mic60 and a significantly lower value of $\sim 20 \mathrm{~nm}$ for Mic19-Mic60. This measurement is in agreement with biochemistry experiments that demonstrate, that Mic10 belongs to a different MICOS subcomplex than Mic60, whereas Mic19 resides in the same subcomplex [31, 39, 116]. An interpretation of absolute distance values rather than a distance comparison is difficult, as the size of the antibodies in combination with the stochastic labeling of the antibodies alter the measured distances. If a more quantitative labeling approach was used, we would further need to compensate the bias in the distance estimation based on the 
nearest-neighbor distribution. Taking these uncertainties into account, our data still suggest that, different to Mic60, the Mic10 molecules do not reside exclusively in small well-defined areas at the center of the crista junctions. This is remarkable as previous studies in yeast found Mic10 oligomerization to be responsible for membrane curvature. Hence, it was expected that Mic10 is located at the highly curved membrane regions that are present in the center of the crista junction [7, 13]. Based on an evaluation of the clustering error, we observed the importance of localization precision and molecular density for the reliability of the molecular assignment and further analysis. As the clustering errors decreased with increased precision, improving the localization precision is very desirable. In Chapter 3, we showed experimental 3D MINFLUX data reaching localization errors of 2-3 nm. In acquisitions of mitochondrial proteins we only reached a precision of $\sim 5 \mathrm{~nm}$. One possible reason for the increased localization errors are the lower SBR values obtained in mitochondrial imaging compared to the imaging of sparser structures close to the cell membrane as shown in Chapter 3. Another source of error are movements in the sample, either due to an incomplete fixation or from movements of the non-covalently bound antibodies. A potential source of background is increased autofluorescence induced when focusing inside the cell. Autofluorescence background could be reduced by using a time-gated detection. A more severe source of background is the uncontrolled chemical interaction of the employed fluorescent molecules with their surrounding. Using a reducing agent, Alexa Fluor 647 can be transferred to a long-lived non-fluorescent triplet state by illumination with excitation light [113]. Under ideal conditions, the lifetime of the non-fluorescent state is on the order of hours, so that the molecules can be activated to fluoresce in a very controlled manner using UV illumination. In reality, the transition probability from the non-fluorescent triplet state to the singlet ground state strongly depends on the local environment of the dyes. Under certain conditions we also observed molecules to photo-activate by illumination with excitation light. Additionally, thermally induced transitions can occur. The described processes lead to spontaneously occurring molecular events inside and outside the MINFLUX imaging region. This can lead to events overlapping in time that induce shifted localizations. If these are not filtered out as described in Section 2.3.3, they induce larger localization errors. This problem can be approached by further engineering the buffer conditions according to the molecular environment. The approach of buffer engineering is very time-consuming, however, as different conditions are necessary for each new sample preparation. Moreover, the range that the buffer optimization offers might not be sufficient for certain sample conditions. More promising is the use of photo-switchable or photo-convertible probes (e.g. [91]) with more stable non-fluorescent states. This change of probe is facilitated by the improved photon efficiency of MINFLUX, which relaxes the constraints on the photon budget of the employed dyes. Another important limitation faced in the presented measurements is the lack of an efficient labeling strategy. Despite their large size compared to the localization precision and the unknown 
stoichiometry of the fluorophore decoration, primary labeled antibodies were still the most suitable available labeling approach. Our attempts for stable homozygous expression of a fusion protein of Mic60 with a protein tag (e.g. SNAP- or HALO-tag) remained unsuccessful so far. This might be due to the interaction of Mic60 with a number of proteins in the outer membrane [87] that can be easily impaired by protein modification. Possible approaches can be the use of much smaller peptide tags, like the ALFA-tag that can be targeted with nanobodies after expression [36]. Reducing the size of the label as well as improving the stability of the non-fluorescent state of the employed dyes can allow for improved apparent labeling efficiencies that simplify the interpretation of the acquired data. When working with resolution at the nanometer-scale, also fixation artifacts need to be considered. A careful comparison of different fixation techniques with respect to their ability to preserve cellular structures at the nanoscale would be required to reduce the uncertainty of biological MINFLUX measurements in fixed cells. Even more desirable is to image living cells. Mitochondrial components like the crista junctions are highly dynamic structures [65], however, so that strongly reduced acquisition times would be required. This could be achieved by using more controlled fluorescent dyes with fewer emission events and by parallelizing the MINFLUX image acquisition. Reduced acquisition times would further facilitate biological studies on a larger scale.

In summary, we could show that the improved and isotropic 3D resolution of MINFLUX can indeed be used to observe protein structures within an organelle at the nanoscale. In combination with the presented novel data analysis approach for the molecular identification of localizations, this can pave the way for quantitative studies of protein arrangements. It is of crucial importance, however, to keep uncertainties in mind that are introduced by the probe, the labeling strategy or the chemical treatment of the cells. 



\section{CONCLUSION}

Within this thesis, I extended the MINFLUX approach for use in quantitative biological studies. To this end, I developed combined 3D and multicolor imaging as well as a multicolor tracking approach. I established a framework for quantitative analysis of MINFLUX imaging data that I applied to the first dual color 3D MINFLUX acquisitions of a cellular organelle. I exploited the unprecedented 3D localization precision and multicolor capability of MINFLUX nanoscopy for studying the spatial distribution of proteins in the heterooligomeric MICOS protein complex that is localized at the crista junction in mitochondria.

In contrast to other super-resolution techniques, MINFLUX achieves molecular resolution because it uses the photons emitted by single fluorescent molecules more efficiently without altering the overall fluorescence photon budget. This is achieved by targeting the minimum of an excitation beam to a pattern of coordinates as close to the fluorescent molecule as possible. In this way, the photon-load is partially shifted from the weak fluorescence emission to the excitation beam that is inherently unlimited in photon-budget. The separation $L$ of the beam target positions serves as a ruler for the single molecule localization, so that the photon efficiency can be tuned by modifying $L$. This happens at the cost of a smaller photon-efficient region. Depending on the exact localization scheme, the localization precision can additionally be anisotropic and spatially inhomogeneous. In contrast to standard camera-based localization approaches, the MINFLUX localization precision does not show a substantial dependence on the wavelength nor on the numerical aperture of the microscope [6]. Furthermore, by localizing emitters purely based on the shape and position of an excitation beam, MINFLUX with a single excitation wavelength is completely void of any chromatic aberrations. MINFLUX is additionally insensitive to the orientation of the emission dipoles, which can lead to substantial artifacts in standard camera-based localization schemes [27, 69]. The best achievable photon efficiency depends on the signal-to-background ratio of the localization. By adjusting the pinhole size in the confocal detection, however, the signal-to-background ratio of the localization can be tuned. The background can be further reduced based 
on a time-gated detection. The temporal resolution of the single-pixel detectors employed for MINFLUX localization can additionally be exploited to obtain fluorescence lifetime information. In its static form with predefined target positions for the illumination beams, the photon-efficient region of MINFLUX localization is limited to the region covered by the target coordinates. Any localization in extended regions requires the online estimation of the position of the molecule and subsequent repositioning of the targeted coordinate pattern (TCP). A first implementation of a self-adapting MINFLUX approach was used to track the position of individual molecules in space and time [6]. Recently, iterative MINFLUX allowed imaging in regions of several micrometers in size by scanning the TCP and the photo-activation beam over the sample while zooming onto individually appearing molecules. Iterative MINFLUX facilitated first proof-of-concept (live) cell imaging with isotropic and homogeneous nanometer localization precision in two dimensions [43].

I extended the photon-efficient localization of MINFLUX to 3D by probing the position of the molecule with an intensity minimum confined in all three dimensions. The minimum was generated using an annular phase mask [63]. An electro-optical lens targeted the beam to coordinates along the optical axis. The lower bounds on the errors for a static 3D localization under experimental conditions were studied. I found that 3D MINFLUX using a cross-like symmetric TCP of size $L=100 \mathrm{~nm}$ can deliver 1-2 $\mathrm{nm}$ localization precision even for an SBR value as low as 2 using $N=2000$ photons. Iterative 3D MINFLUX outperformed the theoretical bounds of any standard camera-based localization scheme in simulations, including coherently used two-objective arrangements. The iterative approach further allowed the imaging of regions larger than the TCP size while preserving an isotropic and homogeneous localization precision in all dimensions. Proof-of-concept measurements of the nuclear pore complex protein Nup96 in U-2 OS cells and PSD-95 in cultured hippocampal neurons demonstrated an almost isotropic localization precision of around $2-3 \mathrm{~nm}$ in a micrometer-sized field of view. MINFLUX clearly resolved the nucleoplasmic and cytoplasmic layer of Nup96. It was, however, not possible to distinguish pairs of proteins residing in $12 \mathrm{~nm}$ distance. This is most likely due to missing labels or an interaction between the molecules located within nanometer vicinity. The observed PSD-95 distribution suggests the agglomeration of C-terminal ends of PSD-95 across the post-synapse with the agglomeration sites occurring in distances of about $40 \mathrm{~nm}$. I used a total maximal number of 2750 photons for each 3D localization, which included the iterative zooming onto the molecule. Assuming the same number of detected photons, an interferometric 4Pi camera-based approach can, in the quantum theoretical limit and in the absence of background, localize with an error of $1.3 \mathrm{~nm}$ laterally and $0.95 \mathrm{~nm}$ axially (see Equation 1.6) [5]. 3D MINFLUX reached a similar regime with an experimental error 2-3 times larger than the quantum theoretical bound of an interferometric 4Pi camera approach. MINFLUX does not rely on an interferometric detection with two objective lenses. This is beneficial because the 
4Pi detection is technically challenging to implement and additionally limits the thickness of the samples that can be observed. Due to technical reasons that are discussed later in this chapter, experimental 3D MINFLUX has so far not reached the Cramér Rao bound. In addition, the experimental parameters, including the control of the intensity distribution of the excitation beam as well as background contributions, have not been fully optimized yet, so that in the near future further improvements in precision are expected.

Single-color fluorescence images, despite of molecular resolution in all dimensions, can only provide insight on the distribution of a single biological compound. In many cases, the function of bio-molecules arises from their reciprocal action with partners in the cell. I applied a multicolor concept well known from stochastic optical reconstruction microscopy (STORM) imaging [125] for MINFLUX multicolor acquisitions. I excited all molecular species with light of $642 \mathrm{~nm}$ wavelength and exploited a spectral shift in their fluorescence emission for emitter classification. For this, I used two spectrally different confocal detection channels. By using a single excitation line, this approach is completely void of chromatic shifts between the localizations of different species. Based on a principal component analysis of the photon counts detected in the different MINFLUX iterations, I distinguished the species with high fidelity. In a 3D acquisition of the NPC, I observed a slightly reduced precision of $2-4 \mathrm{~nm}$ compared to a single-color image. I attribute this to a lower SBR caused by a higher number of fluorescent molecules present in the illuminated region. I further observed that the red-shifted dyes CF660C and CF680 delivered slightly higher errors, most likely related to their lower signal levels due to less efficient excitation.

Using the same fluorescent molecules in a coherent 4Pi STORM approach, resolutions of around 10-20 nm were achieved in imaging of different structures in fixed cells [124]. At the expense of longer acquisition times and reduced image volumes, 3D multicolor MINFLUX with a single objective thus surpasses even this 4Pi localization approach in resolution.

In contrast to MINFLUX tracking, the consecutive registration of single molecules in MINFLUX nanoscopy hampers the observation of dynamic processes in living environments. Here, I developed a multicolor MINFLUX tracking approach that can be used to follow the position of several molecules at the same time. I chose an approach void of chromatic aberrations by using only a single excitation wavelength. This required the simultaneous localization of emitters with potentially overlapping emission spectra. I observed that the spectral overlap increased the lower bounds and worsened the localization performance of the position estimator compared to the single emitter case [6, 26]. I could still correctly recover the distance of $24 \mathrm{~nm}$ between two immobile molecules with a sample size of 12 measurements. By using about 4000 photons per localization, I obtained time bins of $50 \mathrm{~ms}$ length and about 80 localizations per molecule pair. The localizations for each molecule showed an 
average spread of $2-5 \mathrm{~nm}$. It is promising to further enhance the temporal resolution by developing an unbiased and efficient estimator for lower photon numbers or screen the available dyes for reduced spectral overlap. In this way, MINFLUX multicolor tracking is poised to outperform other approaches that were either limited to the observation of static molecules [80] or achieved about 500-3000 ms temporal resolution [84]. MINFLUX can thus deliver a new tool for the observation of dynamic biological processes on length scales not accessible by any other microscopy technique or fluorescence resonance energy transfer.

I further explored the applicability of 3D multicolor MINFLUX imaging for the observation of complex and dense protein structures inside organelles. An almost isotropic localization precision of about $5 \mathrm{~nm}$ was achieved for both molecular species when labeling two different domains of the inner mitochondrial membrane in human dermal fibroblasts. I acquired 3D localizations from single mitochondria over a length of about $10 \mu \mathrm{m}$. I developed an unsupervised-machine-learning-based data analysis framework for the molecular assignment of localizations in a 2D or 3D MINFLUX acquisition. This data analysis framework allowed to study components of single heterooligomeric MICOS protein complexes, located at the crista junctions. I compared the distance between Mic60, the core component of the complex, and Mic10 or Mic19, each representing a different subunit of the MICOS complex. I deduced that Mic10 resides significantly farther away from Mic60 than Mic19. This is in agreement with other results showing that Mic19 belongs to the same sub-complex as Mic60 [31, 39, 116]. Taken together with the MINFLUX data indicating that Mic60 surrounds and thereby forms single crista junctions, the distance measurements suggest that Mic10 molecules do not exclusively reside in a well-defined region at the center of crista junctions. This is noteworthy because previous studies in yeast assigned Mic10 a role in membrane bending and consequently expected Mic10 to be located at the center of the pore $[7,13]$.

Especially in the imaging of mitochondrial structures, two factors were limiting the quality of the resulting datasets: the state control of the employed dyes and the labeling approach. Iterative MINFLUX only acquired molecules appearing within the region of photo-activation. Instabilities in the dark state of the fluorescent molecules introduced background emission because fluorescent molecules outside the TCP were still excited. If molecules got activated outside the TCP, the acquired photons were unusable and filtered out in post-processing. This led to a two-fold loss of localizations, as neither the randomly appearing molecule nor the initially activated molecule were properly captured, resulting in a reduced apparent labeling efficiency. The probability of random activation or activation by the excitation light depends on the labeling density as well as on the local environment of the dyes. High labeling densities can additionally lead to interaction between the fluorescent molecules, meaning that a single emitting molecule can activate molecules that are close-by. This is visible as a step in the photon count trace, but the localization 
is not easily filtered out as described in Section 2.3.3 if the molecular distance is much smaller than the TCP size $L$. The resulting localization reflects the average position of the involved emitters, weighted by the individual molecular brightness, so that the localization precision is in fact limited to the interaction range of the fluorescent molecules. The described effects are more severe for CF660C and CF680 as these dyes display less stable off-states compared to Alexa Fluor 647. Using the listed fluorescent dyes additionally impedes live-cell imaging, as the molecules can not permeate through the intact cell membrane. These limitations could be improved on by exploring different markers for MINFLUX nanoscopy. Examples are photo-switchable dyes [91] or the use of proteins with a photo-induced change in emission state, including photo-conversion, photo-switching or photo-activation [24]. Photo-convertible proteins have already facilitated MINFLUX imaging of the NPC in living mammalian cells in two dimensions [43] and single-molecule tracking in living bacteria [6], but have not been used for 3D imaging so far. Also negatively reversibly switching fluorescent protein variants or uncoupled reversibly switching proteins as reviewed in [24] might prove useful for MINFLUX imaging of densely labeled structures. The photo-physical properties at the single molecule level, however, remain to be explored for many of the variants. Combining MINFLUX with DNA-PAINT [61] could further enhance the achievable labeling efficiency. This approach would also allow the imaging of a higher number of species in MINFLUX. To date, the switching properties of the employed molecules impede the observation of more species with MINFLUX, whereas the current detection scheme can accommodate for the classification of three or more distinct molecular species.

The labeling efficiency is further influenced by the labeling strategy [29, 95]. If aiming for quantitative microscopy, three aspects of the labeling approach have to be considered. The labeling stoichiometry describes how many fluorescent molecules decorate a single target molecule. The size of the label determines how large the bias in the resulting localization will be. Finally, the affinity of the labeling molecule regulates what fraction of the target molecules are actually captured and determines the level of unspecific labeling. Ideally, we aim for a 1:1 decoration as close as possible to the molecule of interest. Moreover, we aim for a labeling approach that does not require fixation or permeabilization of the structure of interest, as these treatments can introduce additional systematic errors. These errors can become relevant when imaging with molecular resolution. Also here, the homozygous endogenous expression of a fusion of the target protein with a suitable fluorescent protein is an auspicious strategy, as it promises a 1:1 labeling stoichiometry by design, assures close proximity of marker and target protein and does not require any fixation.

Next to the discussed aspects of sample preparation including fixation, labeling and especially the choice of fluorescent markers, the performance of 3D MINFLUX was hindered by the focusing capability of the electro-optic lens, limiting the observable volume to about $400 \mathrm{~nm}$ axial extent. Extending this range with different defocusing 
approaches, e.g. a deformable mirror or by stage-scanning, would additionally require a different beam shape, e.g. a Gaussian shaped beam for the first axial iteration. Using a different defocusing device would also be beneficial for an even better control of the excitation beam shape that was observed to deteriorate over hours, presumably due to accumulation of charges in the electro-optic crystal. To achieve an even higher localization precision, we further deem it necessary to measure and employ the true experimental PSF in the analysis.

The acquisition of the datasets shown in this work took tens of minutes to almost two hours. The achieved precision thus requires a reliable stabilization system. In this work, we used the total internal reflection and a dark field image of scattering nanorods for measuring the sample position in $z$ and $x y$ respectively. Both required the illumination of the sample with an infra-red laser beam. Especially in the $x y$ sample lock system, we observed background signal due to scattering of the infra-red illumination from cellular components. This background signal impaired the MINFLUX imaging in some regions, thus limiting the choice of the region of interest. Long acquisition times can also be a limiting factor for biological applications, as acquiring enough samples for drawing a biological conclusion can be overly time consuming. A possible solution is the parallelized implementation of the MINFLUX concept similarly to RESOLFT nanoscopy [14]. Parallelization of the MINFLUX approach is facilitated by the low laser powers employed in the acquisition.

For further enhancement of the photon efficiency in biological imaging, a reduction of the background signal would be necessary. This is because the background determines the minimal beam separation $L$, for which the photon efficiency is still improved [26]. Possible approaches for background reduction include a time-gated detection scheme or the exploration of more efficient beam shapes for excitation. These improvements in combination with a two-photon activation and the development of 3D live-cell imaging can facilitate the observation of structures or dynamics in living tissue. The photon-efficient 3D MINFLUX localization can also be exploited for tracking applications. With a further extension of the MINFLUX multicolor tracking approach to three dimensions, MINFLUX could become a possible alternative to single molecule FRET experiments, delivering absolute positions and the distance between molecules independent of the dipole orientation of the observed emitters. Correlating electron microscopy with MINFLUX could further combine the specificity and accuracy of MINFLUX with the cellular context obtained in electron microscopy.

By developing a 3D and multicolor modality as well as a data analysis framework for the molecular assignment of localizations, I paved the way for quantitative biological imaging with molecular resolution. I expect that this new regime of fluorescence microscopy will allow to answer longstanding questions in biology. It is important to keep in mind, however, that to fully exploit the molecular resolution, each step in the sample preparation procedure must preserve the structure of interest at the 
nanometer scale and ensure close proximity of the target structure and the marker molecule. The best that fluorescence microscopy can do is to correctly map the present fluorophores with molecular accuracy. 



\section{BIBLIOGRAPHY}

[1] E. Abbe. "Beiträge Zur Theorie Des Mikroskops Und Der Mikroskopischen Wahrnehmung". In: Archiv für mikroskopische Anatomie 9.1 (1873), pp. 413418.

[2] A. K. Alkhaja, D. C. Jans, M. Nikolov, M. Vukotic, O. Lytovchenko, F. Ludewig, W. Schliebs, D. Riedel, H. Urlaub, S. Jakobs, and M. Deckers. "MINOS1 Is a Conserved Component of Mitofilin Complexes and Required for Mitochondrial Function and Cristae Organization". In: Molecular Biology of the Cell 23.2 (2012), pp. 247-257.

[3] J. Antelman, C. Wilking-Chang, S. Weiss, and X. Michalet. "Nanometer Distance Measurements between Multicolor Quantum Dots". In: Nano Letters 9.5 (2009), pp. 2199-2205.

[4] D. Aquino, A. Schönle, C. Geisler, C. v Middendorff, C. A. Wurm, Y. Okamura, T. Lang, S. W. Hell, and A. Egner. "Two-Color Nanoscopy of Three-Dimensional Volumes by 4Pi Detection of Stochastically Switched Fluorophores". In: Nature Methods 8.4 (2011), pp. 353-359.

[5] M. P. Backlund, Y. Shechtman, and R. L. Walsworth. "Fundamental Precision Bounds for Three-Dimensional Optical Localization Microscopy with Poisson Statistics". In: Physical Review Letters 121.2 (2018).

[6] F. Balzarotti, Y. Eilers, K. C. Gwosch, A. H. Gynnå, V. Westphal, F. D. Stefani, J. Elf, and S. W. Hell. "Nanometer Resolution Imaging and Tracking of Fluorescent Molecules with Minimal Photon Fluxes". In: Science (2016).

[7] M. Barbot, D. C. Jans, C. Schulz, N. Denkert, B. Kroppen, M. Hoppert, S. Jakobs, and M. Meinecke. "Mic10 Oligomerizes to Bend Mitochondrial Inner Membranes at Cristae Junctions". In: Cell Metabolism 21.5 (2015), pp. $756-763$.

[8] M. Bates, B. Huang, G. T. Dempsey, and X. Zhuang. "Multicolor SuperResolution Imaging with Photo-Switchable Fluorescent Probes". In: Science 317.5845 (2007), pp. 1749-1753. 
[9] S. Berning, K. I. Willig, H. Steffens, P. Dibaj, and S. W. Hell. "Nanoscopy in a Living Mouse Brain". In: Science 335.6068 (2012), p. 551.

[10] E. Betzig, G. H. Patterson, R. Sougrat, O. W. Lindwasser, S. Olenych, J. S. Bonifacino, M. W. Davidson, J. Lippincott-Schwartz, and H. F. Hess. "Imaging Intracellular Fluorescent Proteins at Nanometer Resolution". In: Science 313.5793 (2006), pp. 1642-1645.

[11] N. Bobroff. "Position Measurement with a Resolution and Noise-limited Instrument". In: Review of Scientific Instruments 57.6 (1986), pp. 1152-1157.

[12] U. Böhm, S. W. Hell, and R. Schmidt. "4Pi-RESOLFT Nanoscopy". In: Nature Communications 7.1 (2016), p. 10504.

[13] M. Bohnert, R. M. Zerbes, K. M. Davies, A. W. Mühleip, H. Rampelt, S. E. Horvath, T. Boenke, A. Kram, I. Perschil, M. Veenhuis, W. Kühlbrandt, I. J. van der Klei, N. Pfanner, and M. van der Laan. "Central Role of Mic10 in the Mitochondrial Contact Site and Cristae Organizing System". In: Cell Metabolism 21.5 (2015), pp. 747-755.

[14] A. Chmyrov, J. Keller, T. Grotjohann, M. Ratz, E. d'Este, S. Jakobs, C. Eggeling, and S. W. Hell. "Nanoscopy with More than 100,000 'Doughnuts'". In: Nature Methods 10.8 (2013), pp. 737-740.

[15] L. S. Churchman, Z. Okten, R. S. Rock, J. F. Dawson, and J. A. Spudich. "Single Molecule High-Resolution Colocalization of Cy3 and Cy5 Attached to Macromolecules Measures Intramolecular Distances through Time". In: Proceedings of the National Academy of Sciences 102.5 (2005), pp. 1419-1423.

[16] J. Cnossen, T. Hinsdale, R. Ø. Thorsen, M. Siemons, F. Schueder, R. Jungmann, C. S. Smith, B. Rieger, and S. Stallinga. "Localization Microscopy at Doubled Precision with Patterned Illumination". In: Nature Methods 17.1 (2020), pp. 59-63.

[17] H. Cramér. Mathematical Methods of Statistics, Princeton Univ. Princeton: Princeton University Press, 1946.

[18] E. D'Este, D. Kamin, F. Göttfert, A. El-Hady, and S. W. Hell. "STED Nanoscopy Reveals the Ubiquity of Subcortical Cytoskeleton Periodicity in Living Neurons". In: Cell Reports 10.8 (2015), pp. 1246-1251.

[19] M. Dai, R. Jungmann, and P. Yin. "Optical Imaging of Individual Biomolecules in Densely Packed Clusters". In: Nature Nanotechnology 11.9 (2016), pp. 798807.

[20] W. Denk, J. Strickler, and W. Webb. "Two-Photon Laser Scanning Fluorescence Microscopy". In: Science 248.4951 (1990), pp. 73-76. 
[21] H. Deschout, F. C. Zanacchi, M. Mlodzianoski, A. Diaspro, J. Bewersdorf, S. T. Hess, and K. Braeckmans. "Precisely and Accurately Localizing Single Emitters in Fluorescence Microscopy". In: Nature Methods 11.3 (2014), pp. 253-266.

[22] A. Dlasková, H. Engstová, T. Špaček, A. Kahancová, V. Pavluch, K. Smolková, J. Špačková, M. Bartoš, L. P. Hlavatá, and P. Ježek. "3D Super-Resolution Microscopy Reflects Mitochondrial Cristae Alternations and mtDNA Nucleoid Size and Distribution". In: Biochimica et Biophysica Acta (BBA) Bioenergetics 1859.9 (2018), pp. 829-844.

[23] G. Donnert, J. Keller, C. A. Wurm, S. O. Rizzoli, V. Westphal, A. Schönle, R. Jahn, S. Jakobs, C. Eggeling, and S. W. Hell. "Two-Color Far-Field Fluorescence Nanoscopy". In: Biophysical Journal 92.8 (2007), pp. L67-L69.

[24] S. Duwé and P. Dedecker. "Optimizing the Fluorescent Protein Toolbox and Its Use". In: Current Opinion in Biotechnology 58 (2019), pp. 183-191.

[25] Y. Eilers. "Localizing and Tracking of Fluorescent Molecules with Minimal Photon Fluxes". PhD Thesis. Göttingen: Georg-August University, 2016.

[26] Y. Eilers, H. Ta, K. C. Gwosch, F. Balzarotti, and S. W. Hell. "MINFLUX Monitors Rapid Molecular Jumps with Superior Spatiotemporal Resolution". In: Proceedings of the National Academy of Sciences 115.24 (2018), pp. 61176122 .

[27] J. Engelhardt, J. Keller, P. Hoyer, M. Reuss, T. Staudt, and S. W. Hell. "Molecular Orientation Affects Localization Accuracy in Superresolution FarField Fluorescence Microscopy". In: Nano Letters 11.1 (2011), pp. 209-213.

[28] M. Ester, H.-P. Kriegel, and X. Xu. "A Density-Based Algorithm for Discovering Clusters in Large Spatial Databases with Noise". In: Kdd 96.34 (1996), pp. 226-231.

[29] E. F. Fornasiero and F. Opazo. "Super-Resolution Imaging for Cell Biologists: Concepts, Applications, Current Challenges and Developments". In: Bioessays 37.4 (2015), pp. 436-451.

[30] T. Förster. "Zwischenmolekulare Energiewanderung Und Fluoreszenz". In: Annalen der Physik 437.1-2 (1948), pp. 55-75.

[31] J. R. Friedman, A. Mourier, J. Yamada, J. M. McCaffery, and J. Nunnari. "MICOS Coordinates with Respiratory Complexes and Lipids to Establish Mitochondrial Inner Membrane Architecture". In: eLife 4 (2015), e07739.

[32] Y. Fukata, A. Dimitrov, G. Boncompain, O. Vielemeyer, F. Perez, and M. Fukata. "Local Palmitoylation Cycles Define Activity-Regulated Postsynaptic Subdomains". In: The Journal of Cell Biology 202.1 (2013), pp. 145-161. 
[33] P. Gao, B. Prunsche, L. Zhou, K. Nienhaus, and G. U. Nienhaus. "Background Suppression in Fluorescence Nanoscopy with Stimulated Emission Double Depletion". In: Nature Photonics 11.3 (2017), pp. 163-169.

[34] P. Gao, B. Yao, N. Lindlein, K. Mantel, I. Harder, and E. Geist. "Phase-Shift Extraction for Generalized Phase-Shifting Interferometry". In: Optics letters 34.22 (2009), pp. 3553-3555.

[35] M. P. Gordon, T. Ha, and P. R. Selvin. "Single-Molecule High-Resolution Imaging with Photobleaching". In: Proceedings of the National Academy of Sciences 101.17 (2004), pp. 6462-6465.

[36] H. Götzke, M. Kilisch, M. Martínez-Carranza, S. Sograte-Idrissi, A. Rajavel, T. Schlichthaerle, N. Engels, R. Jungmann, P. Stenmark, F. Opazo, and S. Frey. "The ALFA-Tag Is a Highly Versatile Tool for Nanobody-Based Bioscience Applications". In: Nature Communications 10.1 (2019), p. 4403.

[37] L. Große, C. A. Wurm, C. Brüser, D. Neumann, D. C. Jans, and S. Jakobs. "Bax Assembles into Large Ring-like Structures Remodeling the Mitochondrial Outer Membrane in Apoptosis". In: The EMBO Journal 35.4 (2016), pp. 402413.

[38] L. Gu, Y. Li, S. Zhang, Y. Xue, W. Li, D. Li, T. Xu, and W. Ji. "Molecular Resolution Imaging by Repetitive Optical Selective Exposure". In: Nature Methods 16.11 (2019), pp. 1114-1118.

[39] V. Guarani, E. M. McNeill, J. A. Paulo, E. L. Huttlin, F. Fröhlich, S. P. Gygi, D. Van Vactor, and J. W. Harper. "QIL1 Is a Novel Mitochondrial Protein Required for MICOS Complex Stability and Cristae Morphology". In: eLife 4 (2015).

[40] M. G. L. Gustafsson. "Surpassing the Lateral Resolution Limit by a Factor of Two Using Structured Illumination Microscopy". In: Journal of Microscopy 198.2 (2000), pp. 82-87.

[41] K. C. Gwosch. "Nanometer Resolution Imaging with Minimal Fluorescence Photon Fluxes". PhD Thesis. Heidelberg: Ruperto-Carola University, 2017.

[42] K. C. Gwosch, J. K. Pape, F. Balzarotti, P. Hoess, J. Ellenberg, J. Ries, and S. W. Hell. "MINFLUX Nanoscopy Delivers 3D Multicolor Nanometer Resolution in Cells". In: Nature Methods (2020).

[43] K. C. Gwosch, J. K. Pape, F. Balzarotti, P. Hoess, J. Ellenberg, J. Ries, and S. W. Hell. "MINFLUX Nanoscopy Delivers Multicolor Nanometer 3DResolution in (Living) Cells". In: bioRxiv (2019).

[44] B. Harke, J. Keller, C. K. Ullal, V. Westphal, A. Schönle, and S. W. Hell. "Resolution Scaling in STED Microscopy". In: Opt. Express 16.6 (2008), pp. $4154-4162$. 
[45] M. Harner, C. Körner, D. Walther, D. Mokranjac, J. Kaesmacher, U. Welsch, J. Griffith, M. Mann, F. Reggiori, and W. Neupert. "The Mitochondrial Contact Site Complex, a Determinant of Mitochondrial Architecture: Molecular Architecture of Mitochondria". In: The EMBO Journal 30.21 (2011), pp. $4356-4370$.

[46] R. Heintzmann and C. G. Cremer. "Laterally Modulated Excitation Microscopy: Improvement of Resolution by Using a Diffraction Grating". In: Proc.SPIE. Vol. 3568. 1999.

[47] W. Heisenberg. The Physical Principles of the Quantum Theory. Chicago: Chicago Univ. Press, 1930.

[48] S. Hell, G. Reiner, C. Cremer, and E. H. Stelzer. "Aberrations in Confocal Fluorescence Microscopy Induced by Mismatches in Refractive Index". In: Journal of microscopy 169.3 (1993), pp. 391-405.

[49] S. W. Hell. "Far-Field Optical Nanoscopy". In: Science 316.5828 (2007), pp. $1153-1158$.

[50] S. W. Hell, R. Schmidt, and A. Egner. "Diffraction-Unlimited Three-Dimensional Optical Nanoscopy with Opposing Lenses". In: Nature Photonics 3.7 (2009), pp. 381-387.

[51] S. W. Hell and J. Wichmann. "Breaking the Diffraction Resolution Limit by Stimulated Emission: Stimulated-Emission-Depletion Fluorescence Microscopy". In: Opt. Lett. 19.11 (1994), pp. 780-782.

[52] S. Hell and E. H. K. Stelzer. "Properties of a 4Pi Confocal Fluorescence Microscope". In: Journal of the Optical Society of America A 9.12 (1992), p. 2159.

[53] M. Hessenberger, R. M. Zerbes, H. Rampelt, S. Kunz, A. H. Xavier, B. Purfürst, H. Lilie, N. Pfanner, M. van der Laan, and O. Daumke. "Regulated Membrane Remodeling by Mic60 Controls Formation of Mitochondrial Crista Junctions". In: Nature Communications 8.1 (2017).

[54] S. Hoppins, S. R. Collins, A. Cassidy-Stone, E. Hummel, R. M. DeVay, L. L. Lackner, B. Westermann, M. Schuldiner, J. S. Weissman, and J. Nunnari. "A Mitochondrial-Focused Genetic Interaction Map Reveals a Scaffold-like Complex Required for Inner Membrane Organization in Mitochondria". In: The Journal of Cell Biology 195.2 (2011), pp. 323-340.

[55] F. Huang, G. Sirinakis, E. S. Allgeyer, L. K. Schroeder, W. C. Duim, E. B. Kromann, T. Phan, F. E. Rivera-Molina, J. R. Myers, I. Irnov, M. Lessard, Y. Zhang, M. A. Handel, C. Jacobs-Wagner, C. P. Lusk, J. E. Rothman, D. Toomre, M. J. Booth, and J. Bewersdorf. "Ultra-High Resolution 3D Imaging of Whole Cells". In: Cell 166.4 (2016), pp. 1028-1040. 
[56] S. Jakobs and C. A. Wurm. "Super-Resolution Microscopy of Mitochondria". In: Current Opinion in Chemical Biology 20 (2014), pp. 9-15.

[57] D. C. Jans, C. A. Wurm, D. Riedel, D. Wenzel, F. Stagge, M. Deckers, P. Rehling, and S. Jakobs. "STED Super-Resolution Microscopy Reveals an Array of MINOS Clusters along Human Mitochondria". In: Proceedings of the National Academy of Sciences 110.22 (2013), pp. 8936-8941.

[58] N. Ji, D. E. Milkie, and E. Betzig. "Adaptive Optics via Pupil Segmentation for High-Resolution Imaging in Biological Tissues". In: Nature methods 7.2 (2010), pp. 141-147.

[59] S. Jia, J. C. Vaughan, and X. Zhuang. "Isotropic Three-Dimensional SuperResolution Imaging with a Self-Bending Point Spread Function". In: Nature Photonics 8.4 (2014), pp. 302-306.

[60] R. Jungmann, M. S. Avendano, J. B. Woehrstein, M. Dai, W. M. Shih, and P. Yin. "Multiplexed 3D Cellular Super-Resolution Imaging with DNA-PAINT and Exchange-PAINT". In: Nature methods 11.3 (2014), pp. 313-318.

[61] R. Jungmann, C. Steinhauer, M. Scheible, A. Kuzyk, P. Tinnefeld, and F. C. Simmel. "Single-Molecule Kinetics and Super-Resolution Microscopy by Fluorescence Imaging of Transient Binding on DNA Origami". In: Nano Letters 10.11 (2010), pp. 4756-4761.

[62] H. Kao and A. Verkman. "Tracking of Single Fluorescent Particles in Three Dimensions: Use of Cylindrical Optics to Encode Particle Position". In: Biophysical Journal 67.3 (1994), pp. 1291-1300.

[63] T. A. Klar, S. Jakobs, M. Dyba, A. Egner, and S. W. Hell. "Fluorescence Microscopy with Diffraction Resolution Barrier Broken by Stimulated Emission". In: Proceedings of the National Academy of Sciences 97.15 (2000), pp. 8206-8210.

[64] E. Klotzsch, A. Smorodchenko, L. Löfler, R. Moldzio, E. Parkinson, G. J. Schütz, and E. E. Pohl. "Superresolution Microscopy Reveals Spatial Separation of UCP4 and $\mathrm{F}_{0} \mathrm{~F}_{1}$-ATP Synthase in Neuronal Mitochondria". In: Proceedings of the National Academy of Sciences 112.1 (2015), pp. 130-135.

[65] A. K. Kondadi, R. Anand, S. Hänsch, J. Urbach, T. Zobel, D. M. Wolf, M. Segawa, M. Liesa, O. S. Shirihai, S. Weidtkamp-Peters, and A. S. Reichert. "Cristae Undergo Continuous Cycles of Fusion and Fission in a MICOSDependent Manner". In: bioRxiv (2019).

[66] V. Kozjak-Pavlovic. "The MICOS Complex of Human Mitochondria". In: Cell and Tissue Research 367.1 (2017), pp. 83-93. 
[67] T. D. Lacoste, X. Michalet, F. Pinaud, D. S. Chemla, A. P. Alivisatos, and S. Weiss. "Ultrahigh-Resolution Multicolor Colocalization of Single Fluorescent Probes". In: Proceedings of the National Academy of Sciences 97.17 (2000), pp. 9461-9466.

[68] M. Leutenegger, R. Rao, R. A. Leitgeb, and T. Lasser. "Fast Focus Field Calculations". In: Optics express 14.23 (2006), pp. 11277-11291.

[69] M. D. Lew, M. P. Backlund, and W. E. Moerner. "Rotational Mobility of Single Molecules Affects Localization Accuracy in Super-Resolution Fluorescence Microscopy". In: Nano Letters 13.9 (2013), pp. 3967-3972.

[70] B. Li and J. J. Kohler. "Glycosylation of the Nuclear Pore: Glycosylation of the Nuclear Pore". In: Traffic 15.4 (2014), pp. 347-361.

[71] W. Lukosz and M. Marchand. "Optischen Abbildung Unter Überschreitung der Beugungsbedingten Auflösungsgrenze". In: Optica Acta: International Journal of Optics 10.3 (1963), pp. 241-255.

[72] H. D. MacGillavry and C. C. Hoogenraad. "The Internal Architecture of Dendritic Spines Revealed by Super-Resolution Imaging: What Did We Learn so Far?" In: Experimental Cell Research 335.2 (2015), pp. 180-186.

[73] H. D. MacGillavry, Y. Song, S. Raghavachari, and T. A. Blanpied. "Nanoscale Scaffolding Domains within the Postsynaptic Density Concentrate Synaptic AMPA Receptors". In: Neuron 78.4 (2013), pp. 615-622.

[74] J.-M. Masch, H. Steffens, J. Fischer, J. Engelhardt, J. Hubrich, J. KellerFindeisen, E. D'Este, N. T. Urban, S. G. N. Grant, S. J. Sahl, D. Kamin, and S. W. Hell. "Robust Nanoscopy of a Synaptic Protein in Living Mice by Organic-Fluorophore Labeling". In: Proceedings of the National Academy of Sciences 115.34 (2018), E8047-E8056.

[75] L. A. Masullo, A. Bodén, F. Pennacchietti, G. Coceano, M. Ratz, and I. Testa. "Enhanced Photon Collection Enables Four Dimensional Fluorescence Nanoscopy of Living Systems". In: Nature Communications 9.1 (2018), p. 3281.

[76] W. E. Moerner and L. Kador. "Optical Detection and Spectroscopy of Single Molecules in a Solid". In: Physical Review Letters 62.21 (1989), pp. 2535-2538.

[77] D. Moltchanov. "Distance Distributions in Random Networks". In: Ad Hoc Networks 10.6 (2012), pp. 1146-1166.

[78] K. I. Mortensen, L. S. Churchman, J. A. Spudich, and H. Flyvbjerg. "Optimized Localization Analysis for Single-Molecule Tracking and Super-Resolution Microscopy". In: Nature Methods 7.5 (2010), pp. 377-381.

[79] P. R. Nicovich, D. M. Owen, and K. Gaus. "Turning Single-Molecule Localization Microscopy into a Quantitative Bioanalytical Tool". In: Nature Protocols 12.3 (2017), pp. 453-460. 
[80] S. Niekamp, J. Sung, W. Huynh, G. Bhabha, R. D. Vale, and N. Stuurman. "Nanometer-Accuracy Distance Measurements between Fluorophores at the Single-Molecule Level". In: Proceedings of the National Academy of Sciences 116.10 (2019), pp. 4275-4284.

[81] J. K. Pape, T. Stephan, F. Balzarotti, R. Büchner, F. Lange, D. Riedel, S. Jakobs, and S. W. Hell. "Multicolor 3D MINFLUX Nanoscopy of Mitochondrial MICOS Proteins". In: Proceedings of the National Academy of Sciences 117.34 (2020), pp. 20607-20614.

[82] J. B. Pawley and B. R. Masters. "Handbook of Biological Confocal Microscopy, Third Edition". In: Journal of biomedical optics 13.2 (2008), p. 029902.

[83] G. Perkins, C. Renken, M. Martone, S. Young, M. Ellisman, and T. Frey. "Electron Tomography of Neuronal Mitochondria: Three-Dimensional Structure and Organization of Cristae and Membrane Contacts". In: Journal of Structural Biology 119.3 (1997), pp. 260-272.

[84] A. Pertsinidis, Y. Zhang, and S. Chu. "Subnanometre Single-Molecule Localization, Registration and Distance Measurements". In: Nature 466.7306 (2010), pp. 647-651.

[85] N. Pfanner, M. van der Laan, P. Amati, R. A. Capaldi, A. A. Caudy, A. Chacinska, M. Darshi, M. Deckers, S. Hoppins, T. Icho, S. Jakobs, J. Ji, V. Kozjak-Pavlovic, C. Meisinger, P. R. Odgren, S. K. Park, P. Rehling, A. S. Reichert, M. S. Sheikh, S. S. Taylor, N. Tsuchida, A. M. van der Bliek, I. J. van der Klei, J. S. Weissman, B. Westermann, J. Zha, W. Neupert, and J. Nunnari. "Uniform Nomenclature for the Mitochondrial Contact Site and Cristae Organizing System". In: The Journal of Cell Biology 204.7 (2014), pp. 1083-1086.

[86] X. Qu, D. Wu, L. Mets, and N. F. Scherer. "Nanometer-Localized Multiple Single-Molecule Fluorescence Microscopy". In: Proceedings of the National Academy of Sciences 101.31 (2004), pp. 11298-11303.

[87] H. Rampelt, R. M. Zerbes, M. van der Laan, and N. Pfanner. "Role of the Mitochondrial Contact Site and Cristae Organizing System in Membrane Architecture and Dynamics". In: Biochimica et Biophysica Acta (BBA) Molecular Cell Research 1864.4 (2017), pp. 737-746.

[88] R. C. Rao. "Information and Accuracy Attainable in the Estimation of Statistical Parameters". In: Bulletin of the Calcutta Mathematical Society 37.3 (1945), pp. 81-91.

[89] L. Reymond, J. Ziegler, C. Knapp, F.-C. Wang, T. Huser, V. Ruprecht, and S. Wieser. "SIMPLE: Structured Illumination Based Point Localization Estimator with Enhanced Precision”. In: Optics Express 27.17 (2019), p. 24578. 
[90] P. W. K. Rothemund. "Folding DNA to Create Nanoscale Shapes and Patterns". In: Nature 440.7082 (2006), pp. 297-302.

[91] B. Roubinet, M. L. Bossi, P. Alt, M. Leutenegger, H. Shojaei, S. Schnorrenberg, S. Nizamov, M. Irie, V. N. Belov, and S. W. Hell. "Carboxylated Photoswitchable Diarylethenes for Biolabeling and Super-Resolution RESOLFT Microscopy". In: Angewandte Chemie International Edition 128.49 (2016), pp. $15655-15659$.

[92] R. Roy, S. Hohng, and T. Ha. "A Practical Guide to Single-Molecule FRET". In: Nature methods 5.6 (2008), pp. 507-516.

[93] M. J. Rust, M. Bates, and X. Zhuang. "Sub-Diffraction-Limit Imaging by Stochastic Optical Reconstruction Microscopy (STORM)". In: Nature methods 3.10 (2006), pp. 793-796.

[94] M. D. Ryan, A. M. Q. King, and G. P. Thomas. "Cleavage of Foot-and-Mouth Disease Virus Polyprotein Is Mediated by Residues Located within a 19 Amino Acid Sequence". In: Journal of General Virology 72.11 (1991), pp. 2727-2732.

[95] S. J. Sahl, S. W. Hell, and S. Jakobs. "Fluorescence Nanoscopy in Cell Biology". In: Nature Reviews Molecular Cell Biology 18.11 (2017), pp. 685-701.

[96] R. Salvador-Gallego, M. Mund, K. Cosentino, J. Schneider, J. Unsay, U. Schraermeyer, J. Engelhardt, J. Ries, and A. J. García-Sáez. "Bax Assembly into Rings and Arcs in Apoptotic Mitochondria Is Linked to Membrane Pores". In: The EMBO Journal 35.4 (2016), pp. 389-401.

[97] R. Schmidt, C. A. Wurm, S. Jakobs, J. Engelhardt, A. Egner, and S. W. Hell. "Spherical Nanosized Focal Spot Unravels the Interior of Cells". In: Nature Methods 5.6 (2008), pp. 539-544.

[98] J. J. Schmied, M. Raab, C. Forthmann, E. Pibiri, B. Wünsch, T. Dammeyer, and P. Tinnefeld. "DNA Origami-Based Standards for Quantitative Fluorescence Microscopy". In: Nature Protocols 9.6 (2014), pp. 1367-1391.

[99] A. Schönle. Imspector Image Acquisition and Analysis Software, v0. 1. 2006.

[100] A. Sharonov and R. M. Hochstrasser. "Wide-Field Subdiffraction Imaging by Accumulated Binding of Diffusing Probes". In: Proceedings of the National Academy of Sciences 103.50 (2006), pp. 18911-18916.

[101] Y. Shechtman, S. J. Sahl, A. S. Backer, and W. E. Moerner. "Optimal Point Spread Function Design for 3D Imaging". In: Physical Review Letters 113.13 (2014), p. 133902.

[102] Y. Shechtman, L. E. Weiss, A. S. Backer, S. J. Sahl, and W. E. Moerner. "Precise Three-Dimensional Scan-Free Multiple-Particle Tracking over Large Axial Ranges with Tetrapod Point Spread Functions". In: Nano Letters 15.6 (2015), pp. 4194-4199. 
[103] G. Shtengel, J. A. Galbraith, C. G. Galbraith, J. Lippincott-Schwartz, J. M. Gillette, S. Manley, R. Sougrat, C. M. Waterman, P. Kanchanawong, M. W. Davidson, R. D. Fetter, and H. F. Hess. "Interferometric Fluorescent SuperResolution Microscopy Resolves 3D Cellular Ultrastructure". In: Proceedings of the National Academy of Sciences 106.9 (2009), pp. 3125-3130.

[104] H. W. Sorenson. Parameter Estimation: Principles and Problems. Vol. 9. M. Dekker, 1980.

[105] S. Stoldt, T. Stephan, D. C. Jans, C. Brüser, F. Lange, J. Keller-Findeisen, D. Riedel, S. W. Hell, and S. Jakobs. "Mic60 Exhibits a Coordinated Clustered Distribution along and across Yeast and Mammalian Mitochondria". In: Proceedings of the National Academy of Sciences 116.20 (2019), pp. 98539858.

[106] D. Tarasenko, M. Barbot, D. C. Jans, B. Kroppen, B. Sadowski, G. Heim, W. Möbius, S. Jakobs, and M. Meinecke. "The MICOS Component Mic60 Displays a Conserved Membrane-Bending Activity That Is Necessary for Normal Cristae Morphology". In: The Journal of Cell Biology 216.4 (2017), pp. 889-899.

[107] I. Testa, E. D'Este, N. T. Urban, F. Balzarotti, and S. W. Hell. "Dual Channel RESOLFT Nanoscopy by Using Fluorescent State Kinetics". In: Nano letters 15.1 (2014), pp. 103-106.

[108] J. V. Thevathasan, M. Kahnwald, K. Cieśliński, P. Hoess, S. K. Peneti, M. Reitberger, D. Heid, K. C. Kasuba, S. J. Hoerner, Y. Li, Y.-L. Wu, M. Mund, U. Matti, P. M. Pereira, R. Henriques, B. Nijmeijer, M. Kueblbeck, V. J. Sabinina, J. Ellenberg, and J. Ries. "Nuclear Pores as Versatile Reference Standards for Quantitative Superresolution Microscopy". In: Nature Methods 16.10 (2019), pp. 1045-1053.

[109] J. Tønnesen, G. Katona, B. Rózsa, and U. V. Nägerl. "Spine Neck Plasticity Regulates Compartmentalization of Synapses". In: Nature Neuroscience 17.5 (2014), pp. 678-685.

[110] H. A. Tuppen, E. L. Blakely, D. M. Turnbull, and R. W. Taylor. "Mitochondrial DNA Mutations and Human Disease". In: Biochimica et Biophysica Acta (BBA) - Bioenergetics 1797.2 (2010), pp. 113-128.

[111] N. T. Urban, K. I. Willig, S. W. Hell, and U. V. Nägerl. "STED Nanoscopy of Actin Dynamics in Synapses Deep Inside Living Brain Slices". In: Biophysical Journal 101.5 (2011), pp. 1277-1284.

[112] C. v. Middendorff, A. Egner, C. Geisler, S. W. Hell, and A. Schönle. "Isotropic 3D Nanoscopy Based on Single Emitter Switching". In: Optics Express 16.25 (2008), pp. 20774-20788. 
[113] S. van de Linde, A. Löschberger, T. Klein, M. Heidbreder, S. Wolter, M. Heilemann, and M. Sauer. "Direct Stochastic Optical Reconstruction Microscopy with Standard Fluorescent Probes". In: Nature Protocols 6.7 (2011), pp. 991-1009.

[114] J. Vogelsang, R. Kasper, C. Steinhauer, B. Person, M. Heilemann, M. Sauer, and P. Tinnefeld. "A Reducing and Oxidizing System Minimizes Photobleaching and Blinking of Fluorescent Dyes". In: Angewandte Chemie International Edition 47.29 (2008), pp. 5465-5469.

[115] A. von Appen, J. Kosinski, L. Sparks, A. Ori, A. L. DiGuilio, B. Vollmer, M.-T. Mackmull, N. Banterle, L. Parca, P. Kastritis, K. Buczak, S. Mosalaganti, W. Hagen, A. Andres-Pons, E. A. Lemke, P. Bork, W. Antonin, J. S. Glavy, K. H. Bui, and M. Beck. "In Situ Structural Analysis of the Human Nuclear Pore Complex". In: Nature 526.7571 (2015), pp. 140-143.

[116] K. von der Malsburg, J. M. Müller, M. Bohnert, S. Oeljeklaus, P. Kwiatkowska, T. Becker, A. Loniewska-Lwowska, S. Wiese, S. Rao, D. Milenkovic, D. P. Hutu, R. M. Zerbes, A. Schulze-Specking, H. E. Meyer, J.-C. Martinou, S. Rospert, P. Rehling, C. Meisinger, M. Veenhuis, B. Warscheid, I. J. van der Klei, N. Pfanner, A. Chacinska, and M. van der Laan. "Dual Role of Mitofilin in Mitochondrial Membrane Organization and Protein Biogenesis". In: Developmental Cell 21.4 (2011), pp. 694-707.

[117] A. von Diezmann, Y. Shechtman, and W. E. Moerner. "Three-Dimensional Localization of Single Molecules for Super-Resolution Imaging and SingleParticle Tracking". In: Chemical Reviews 117.11 (2017), pp. 7244-7275.

[118] O. K. Wade, J. B. Woehrstein, P. C. Nickels, S. Strauss, F. Stehr, J. Stein, F. Schueder, M. T. Strauss, M. Ganji, J. Schnitzbauer, H. Grabmayr, P. Yin, P. Schwille, and R. Jungmann. "124-Color Super-Resolution Imaging by Engineering DNA-PAINT Blinking Kinetics". In: Nano Letters 19.4 (2019), pp. 2641-2646.

[119] S. Weisenburger, D. Boening, B. Schomburg, K. Giller, S. Becker, C. Griesinger, and V. Sandoghdar. "Cryogenic Optical Localization Provides 3D Protein Structure Data with Angstrom Resolution". In: Nature Methods 14.2 (2017), pp. 141-144.

[120] S. Weisenburger, B. Jing, D. Hänni, L. Reymond, B. Schuler, A. Renn, and V. Sandoghdar. "Cryogenic Colocalization Microscopy for Nanometer-Distance Measurements". In: ChemPhysChem 15.4 (2014), pp. 763-770.

[121] T. Wilson, C. Sheppard, and H. B. Jovanovich. Theory and Practice of Scanning Optical Microscopy. Vol. 180. Academic Press London, 1984. 
[122] K. Xu, G. Zhong, and X. Zhuang. "Actin, Spectrin, and Associated Proteins Form a Periodic Cytoskeletal Structure in Axons". In: Science 339.6118 (2013), pp. $452-456$.

[123] A. Yildiz, J. N. Forkey, S. A. McKinney, T. Ha, Y. E. Goldman, and P. R. Selvin. "Myosin V Walks Hand-Over-Hand: Single Fluorophore Imaging with 1.5-Nm Localization". In: Science 300.5628 (2003), pp. 2061-2065.

[124] Y. Zhang, L. K. Schroeder, M. D. Lessard, P. Kidd, J. Chung, Y. Song, L. Benedetti, Y. Li, J. Ries, J. B. Grimm, L. D. Lavis, P. De Camilli, J. E. Rothman, D. Baddeley, and J. Bewersdorf. "Nanoscale Subcellular Architecture Revealed by Multicolor Three-Dimensional Salvaged Fluorescence Imaging". In: Nature Methods (2020).

[125] Z. Zhang, S. J. Kenny, M. Hauser, W. Li, and K. Xu. "Ultrahigh-Throughput Single-Molecule Spectroscopy and Spectrally Resolved Super-Resolution Microscopy". In: Nature methods 12.10 (2015), pp. 935-938.

[126] X. Zhu, X. Peng, M.-X. Guan, and Q. Yan. "Pathogenic Mutations of Nuclear Genes Associated with Mitochondrial Disorders". In: Acta Biochimica et Biophysica Sinica 41.3 (2009), pp. 179-187. 
APPENDIX FIGURES 

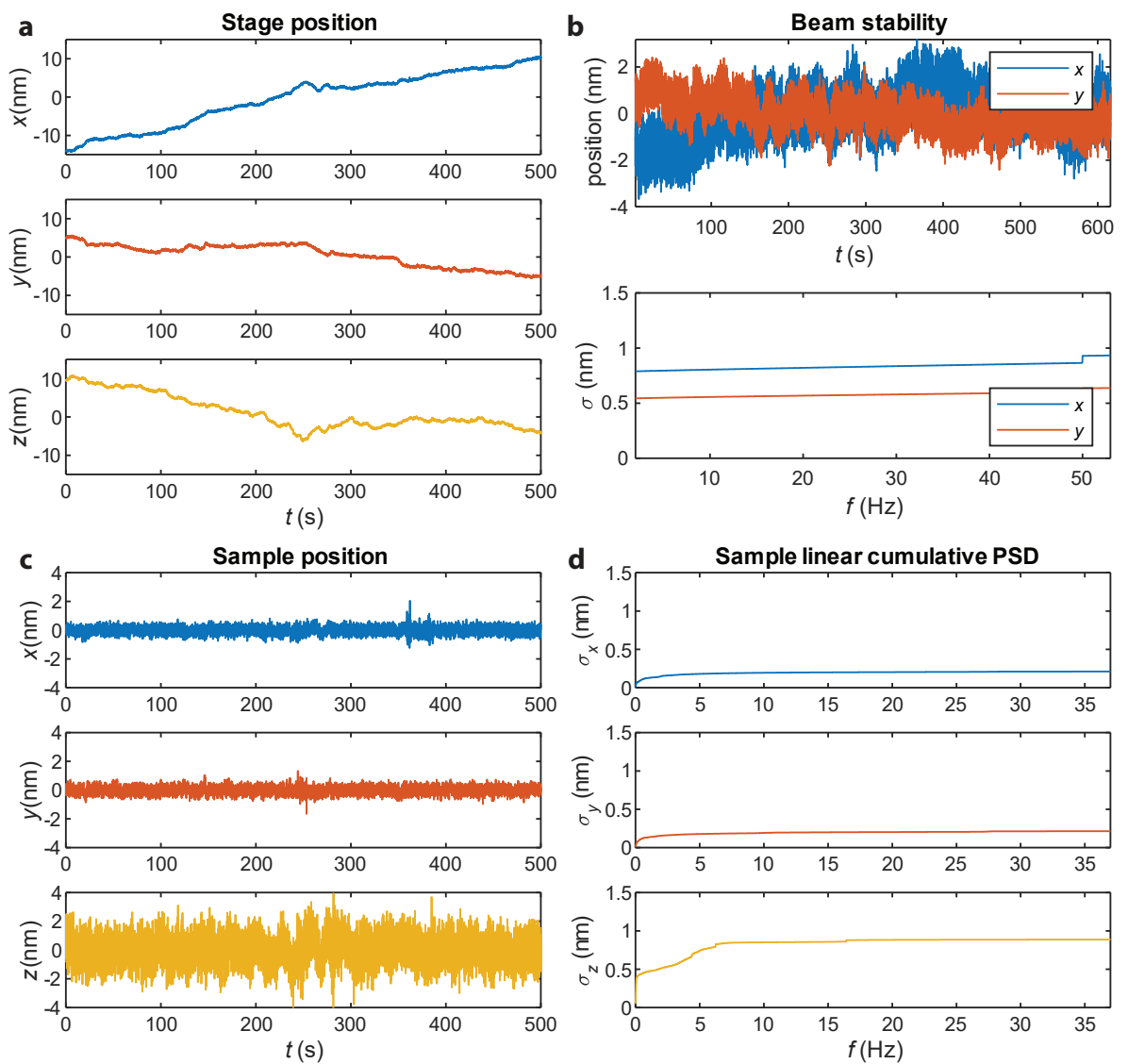

Figure A.1: Measurement of the stability of the imaging system. For measuring the beam stability, we co-aligned a laser of $870 \mathrm{~nm}$ wavelength with the excitation beam exiting the electro-optical deflectors. The beam was partially transmitted through a back-side polished mirror and focused onto a camera. For measuring and actively compensating sample drift along the optical axis, we imaged the reflection of a beam of $905 \mathrm{~nm}$ wavelength in total internal reflection (TIR) incidence at the glass-water interface in the sample. For controlling the lateral position of the sample, we acquired a dark-field image of scattering gold nanorods illuminated with light of 950-1000 nm wavelength. a, Time evolution of the absolute piezo-stage position in $x, y$ and $z$. We observed that the stage compensates sample drift on the order of $2 \mathrm{~nm} / \mathrm{min}$. $\mathbf{b}$, Beam position on the camera in $x$ and $y$ together with the cumulative power spectral density. c, The sample position in $x, y$ and $z$ is kept constant over time by actively compensating for sample drifts. d, Linear cumulative power spectral density of the sample position, indicating sample movements of less than $1 \mathrm{~nm}$ for frequencies below $35 \mathrm{~Hz}$. 

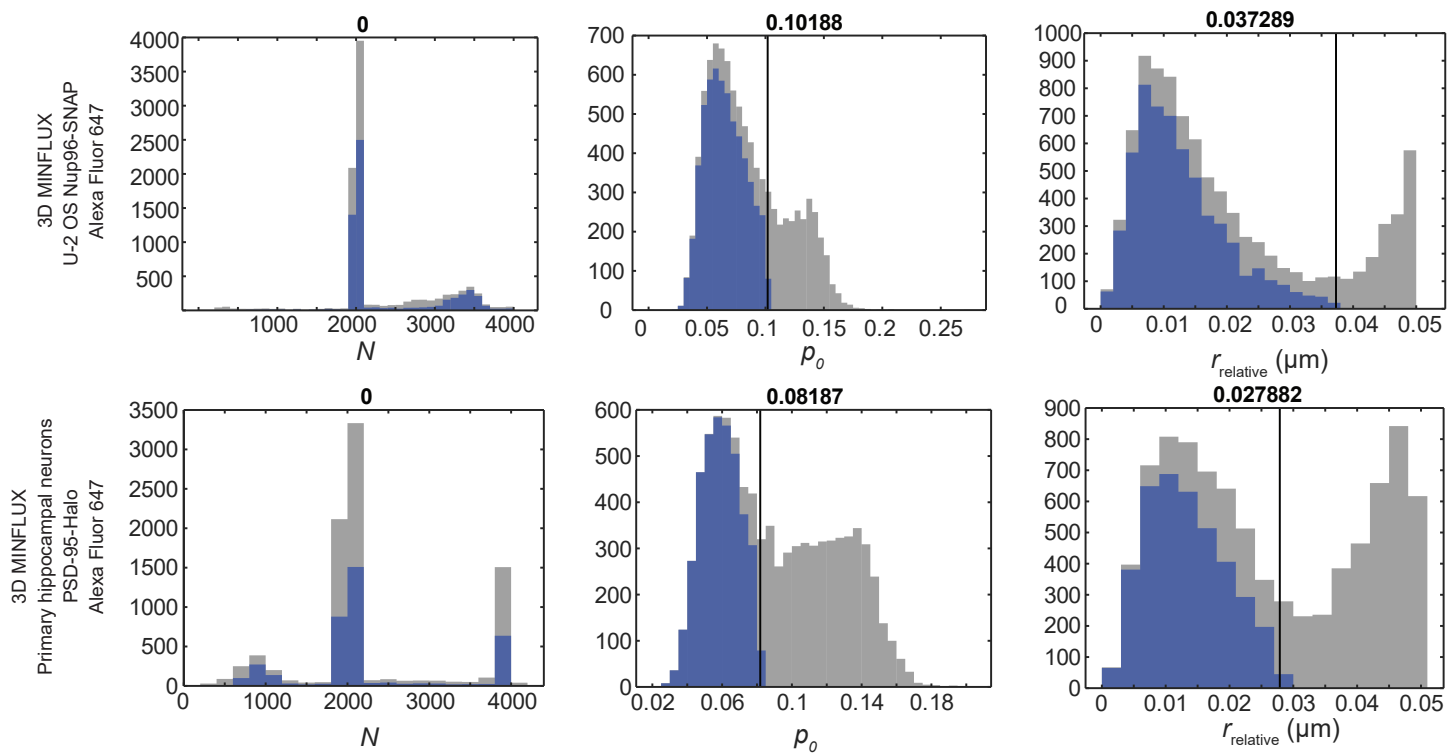

Figure A.2: Filter values and distributions for single-color 3D imaging. Top: distribution of photon numbers in the last iteration $N$, probability of photon detection in the central exposure $p_{0}$ and distance of the localization to the TCP center in the last iteration $r_{\text {relative }}$ for the dataset shown in Figure 3.8. All filters are defined in section 2.3.3. In gray: full distribution. In blue: filtered distribution. Filter values are indicated with black lines and on top of the histograms. Bottom: same as top for dataset in Figure 3.10. Material from: Gwosch, K.C.*, Pape, J.K.*, Balzarotti, F.* et al., MINFLUX nanoscopy delivers 3D multicolor nanometer resolution in cells, Nature Methods, 2020, Springer Nature. 

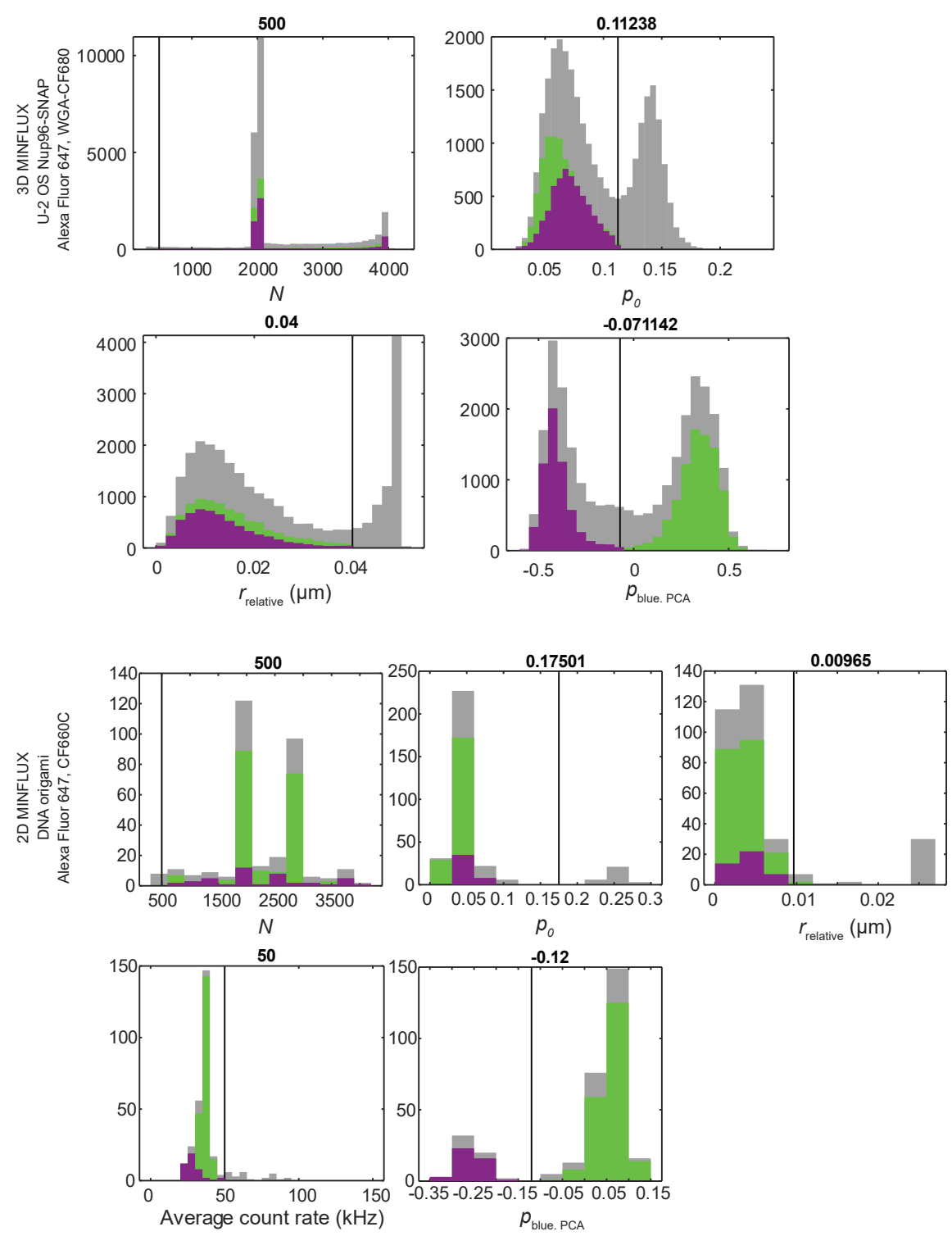

Figure A.3: Filter values and distributions for two-color imaging. Top: distribution of photon numbers in the last iteration $N$, probability of photon detection in the central exposure $p_{0}$, distance of the localization to the TCP center in the last iteration $r_{\text {relative }}$ and first principal component of the count fraction on the detection channel for shorter wavelengths $p_{\text {blue,PCA }}$ used for species classification. Shown for dataset in Figure 4.4. In gray: full distribution. In magenta: filtered distribution of CF680. In green: filtered distribution of Alexa Fluor 647. Filter values are indicated with black lines and on top of the histograms. Bottom: same as top, but for dataset in Figure 4.3. Additionally showing the average count rate of the localizations. In gray: full distribution. In magenta: filtered distribution of CF660C. In green: filtered distribution of Alexa Fluor 647. Material from: Gwosch, K.C.*, Pape, J.K.*, Balzarotti, F.* et al., MINFLUX nanoscopy delivers 3D multicolor nanometer resolution in cells, Nature Methods, 2020, Springer Nature. 

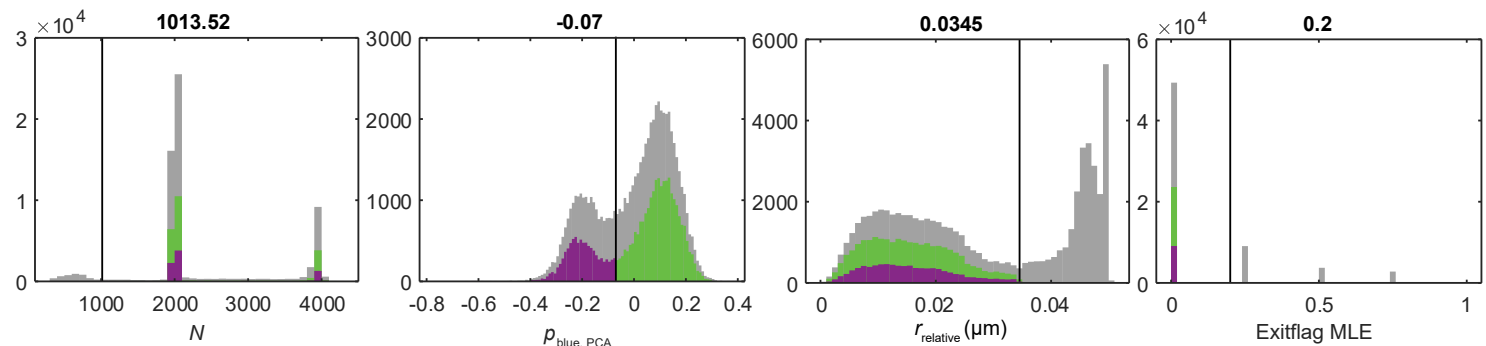

Figure A.4: Filter values and distributions for two-color 3D imaging of mitochondrial proteins. All distributions correspond to the data shown in Figure 5.2. Distribution of photon numbers in the last iteration $N$, first principal component of the count fraction on the detection channel for shorter wavelengths $p_{\text {blue,PCA }}$ used for species classification, distance of the localization to the TCP center in the last iteration $r_{\text {relative }}$ and exitflag of the MLE as introduced in section 2.3.3. Exitflag values larger than 0 indicate coincidence of the localization result with one of the grid edges. In gray: full distribution. In magenta: filtered distribution of CF660C. In green: filtered distribution of Alexa Fluor 647. Filter values are indicated with black lines and on top of the histograms. The same filtering thresholds were used for dual-color images of Mic10 or Mic19 with Mic60 (Figure 5.10). Material from [81].
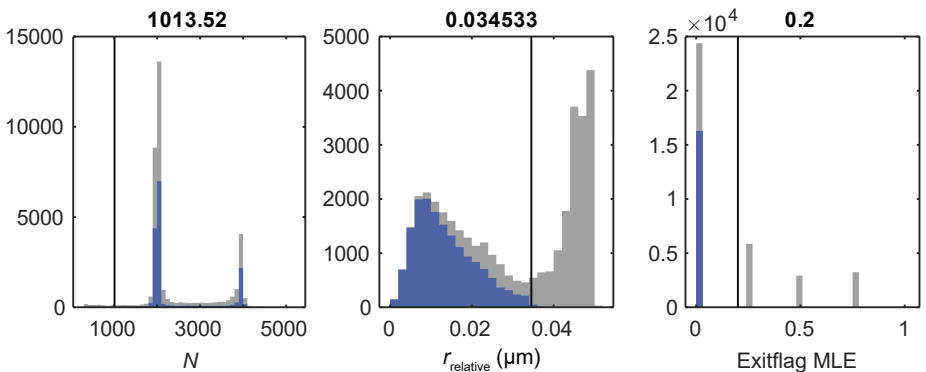

Figure A.5: Filter values and distributions for single-color Mic60 acquisition in U-2 OS cells. All distributions correspond to the data shown in Figure 5.9. Distribution of photon numbers in the last iteration $N$, distance of the localization to the TCP center in the last iteration $r_{\text {relative }}$ and exitflag of the MLE as introduced in section 2.3.3. Exitflag values larger than 0 indicate coincidence of the localization result with one of the grid edges. In gray: full distribution. In blue: filtered distribution of Alexa Fluor 647. Filter values are indicated with black lines and on top of the histograms. Material from [81]. 


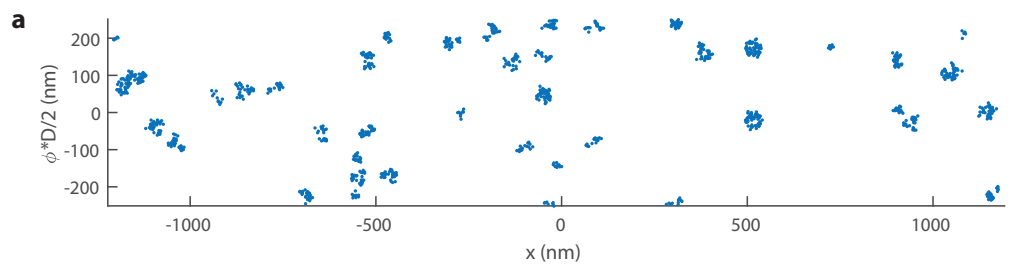

b

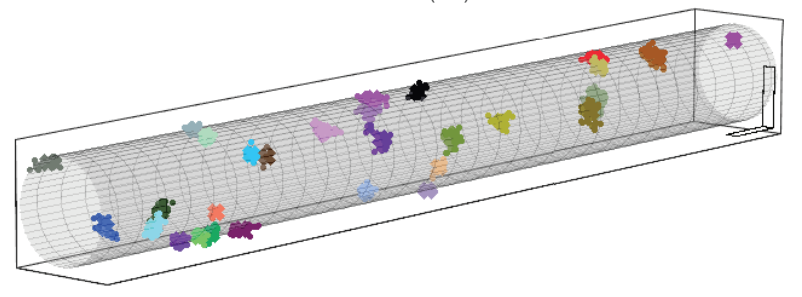

c

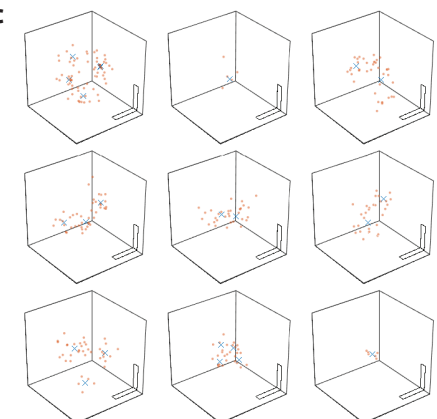

d

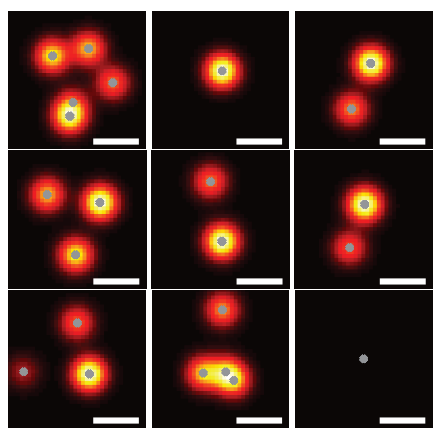

Figure A.6: Simulation of a localizations for a MICOS protein in the crista junction. a, Unwrapped cylinder $(D=160 \mathrm{~nm})$ containing localizations of roughly 80 junctions at a density of 30 junctions $/ \mathrm{\mu m}^{2}$. We assumed a localization precision of $\sigma=5 \mathrm{~nm}$, an average number of 10 localizations per molecule and a labeling efficiency of $50 \%$. We assumed six molecules per junctions equally distributed on a circle with radius $15 \mathrm{~nm}$. b, We isolated single junctions (one color per junction) and molecule positions within a junction by applying the previously described three-step dbscan2 clustering to the simulated localizations. We used the following parameters: $e^{p p s_{\text {loc }}}=[10 \mathrm{~nm}, 6 \mathrm{~nm}], \operatorname{minPts} s_{\mathrm{loc}}=[3,4], \sigma_{\mathrm{gmm}, \mathrm{loc}}=5 \mathrm{~nm}$, $e p s_{\text {junction }}=[25 \mathrm{~nm}, 15 \mathrm{~nm}], \operatorname{minPt} s_{\text {junction }}=[3,10]$ and $\sigma_{\text {gmm,junction }}=20 \mathrm{~nm}$. c, Isolated junctions oriented with the cylinder surface. $\mathbf{d}$, Isolated junctions rotated to the $x y$-plane by using the eigenvectors of the covariance matrix of the localizations. Localizations are rendered as Gaussian distributions with $\sigma=5 \mathrm{~nm}$, identified molecule positions are marked (gray). Scale bars: $100 \mathrm{~nm}(\mathrm{~b}), 20 \mathrm{~nm}(\mathrm{c}, \mathrm{d})$. 
DNA STRANDS 
Biotinylated

Biotin conjugated to $5^{\circ}$ end, HPLC purified

\begin{tabular}{|c|l|}
\hline 47 & CTTTGAAAAGAACTGGCTCATTATTTAATAAA \\
\hline 54 & CCGGAAACACACCACGGAATAAGTAAGACTCC \\
\hline 103 & CGAGTAGAACTAATAGTAGTAGCAAACCCTCA \\
\hline 106 & GGTATTAAGAACAAGAAAAATAATTAAAGCCA \\
\hline 173 & CTTGCATGCATTAATGAATCGGCCCGCCAGGG \\
\hline 180 & CGGAATTATTGAAAGGAATTGAGGTGAAAAAT \\
\hline
\end{tabular}

For Alexa Fluor 647 labeling sites

\begin{tabular}{|c|l|}
\hline 39 & TTATTCCTGTAGTATATGGCAATGAAATTATGCTCCATGAGAGGCTTTGAGGACTAGGGAGTT \\
\hline 41 & TTATTCCTGTAGTATATGGCAATGAAATTATGCGAAACATGCCACTACGAAGGCATGCGCCGA \\
\hline 111 & TTATTCCTGTAGTATATGGCAATGAAATTATCAAAATTAAAGTACGGTGTCTGGAAGAGGTCA \\
\hline 113 & TTATTCCTGTAGTATATGGCAATGAAATTATTCAATTCTTTTAGTTTGACCATTACCAGACCG \\
\hline 130 & TTATTCCTGTAGTATATGGCAATGAAATTATGGTAGCTAGGATAAAAATTTTTAGTTAACATC \\
\hline
\end{tabular}

\section{For CF660C labeling sites}

\begin{tabular}{|c|l|}
\hline 58 & ACTAGCGGCAACGGCCCAACTATCCATTTTTTTCAACTATAGGCTGGCTGACCTTGTATCAT \\
\hline 75 & ACTAGCGGCAACGGCCCAACTATCCATTTTACTGGATAACGGAACAACATTATTACCTTATG \\
\hline 77 & ACTAGCGGCAACGGCCCAACTATCCATTTTCCAAAATATAATGCAGATACATAAACACCAGA \\
\hline 94 & ACTAGCGGCAACGGCCCAACTATCCATTTTTACCTTTAAGGTCTTTACCCTGACAAAGAAGT \\
\hline
\end{tabular}

\section{For ATTO 647N labeling site}

75 TTATTCCTGTAGTATATGGCAATGAAATTATACTGGATAACGGAACAACATTATTACCTTATG

\section{For ATTO 665 labeling site}

$$
77 \text { ACTAGCGGCAACGGCCCAACTATCCATTTTCCAAAATATAATGCAGATACATAAACACCAGA }
$$

\section{Marker strands}

Dye conjugated to 5 ` end, PAGE/HPLC purified

\begin{tabular}{|l|l|}
\hline Alexa Fluor 647/ ATTO 647N & TAATTTCATTGCCATATACTACAGGAATAA \\
\hline CF660C/ATTO 665 & AAATGGATAGTTGGGCCGTTGCCGCTAGT \\
\hline
\end{tabular}

\section{Staple strands}

\begin{tabular}{|c|l|}
\hline 2 & ACGTTAGTAAATGAATTTTCTGTAAGCGGAGT \\
\hline 3 & CGTAACGATCTAAAGTTTTGTCGTGAATTGCG \\
\hline 4 & TGTAGCATTCCACAGACAGCCCTCATCTCCAA \\
\hline 5 & TGAGTTTCGTCACCAGTACAAACTTAATTGTA \\
\hline 6 & CAAGCCCAATAGGAACCCATGTACCGTAACAC \\
\hline 7 & CTCAGAGCCACCACCCTCATTTTCCTATTATT \\
\hline 8 & CCCTCAGAACCGCCACCCTCAGAACTGAGACT \\
\hline 9 & TATCACCGTACTCAGGAGGTTTAGCGGGGTTT \\
\hline 11 & GAGAATAGCTTTTGCGGGATCGTCGGGTAGCA \\
\hline 12 & AATAATAAGGTCGCTGAGGCTTGCAAAGACTT \\
\hline 13 & AAAAAAGGACAACCATCGCCCACGCGGGTAAA \\
\hline 14 & TCGGTTTAGCTTGATACCGATAGTCCAACCTA \\
\hline 15 & AATGCCCCGTAACAGTGCCCGTATGTGAATTT \\
\hline 16 & CTGAAACAGGTAATAAGTTTTAACCCCTCAGA \\
\hline 17 & CCTCAAGAATACATGGCTTTTGATAGAACCAC \\
\hline 18 & TGCTCAGTCAGTCTCTGAATTTACCAGGAGGT \\
\hline
\end{tabular}

\begin{tabular}{|c|l|}
\hline 21 & AAAGGCCGAAAGGAACAACTAAAGCTTTCCAG \\
\hline 22 & ATATATTCTTTTTTCACGTTGAAAATAGTTAG \\
\hline 23 & CAATGACACTCCAAAAGGAGCCTTACAACGCC \\
\hline 24 & CTTAAACATCAGCTTGCTTTCGAGAAACAGTT \\
\hline 25 & TGCCTTGACTGCCTATTTCGGAACAGGGATAG \\
\hline 26 & AGTGTACTTGAAAGTATTAAGAGGCCGCCACC \\
\hline 27 & TAAGCGTCGAAGGATTAGGATTAGTACCGCCA \\
\hline 28 & GGAAAGCGACCAGGCGGATAAGTGAATAGGTG \\
\hline 29 & ACGGCTACTTACTTAGCCGGAACGCTGACCAA \\
\hline 30 & TTTCATGAAAATTGTGTCGAAATCTGTACAGA \\
\hline 31 & ATACGTAAAAGTACAACGGAGATTTCATCAAG \\
\hline 32 & AAACGAAATGACCCCCAGCGATTATTCATTAC \\
\hline 33 & GAGCCGCCCCACCACCGGAACCGCCTAAAACA \\
\hline 34 & GCCACCACTCTTTTCATAATCAAACCGTCACC \\
\hline 35 & CACCAGAGTTCGGTCATAGCCCCCGCCAGCAA \\
\hline 36 & TGAGGCAGGCGTCAGACTGTAGCGTAGCAAGG \\
\hline
\end{tabular}




\begin{tabular}{|c|c|}
\hline 39 & $\begin{array}{l}\text { GCTCCATGAGAGGCTTTGAGGACTAGGGAGTT } \\
\text { Modified > Alexa Fluor } 647\end{array}$ \\
\hline 40 & CGCCTGATGGAAGTTTCCATTAAACATAACCG \\
\hline 41 & $\begin{array}{l}\text { GCGAAACATGCCACTACGAAGGCATGCGCCGA } \\
\text { Modified > Alexa Fluor } 647\end{array}$ \\
\hline 42 & СTCATCTTGAGGCAAAAGAATACACTCCCTCA \\
\hline 43 & AACCAGAGACCCTCAGAACCGCCAGGGGTCAG \\
\hline 44 & GTTTGCCACCTCAGAGCCGCCACCGATACAGG \\
\hline 45 & TCGGCATTCCGCCGCCAGCATTGACGTTCCAG \\
\hline 46 & TGCCTTTAGTCAGACGATTGGCCTGCCAGAAT \\
\hline 47 & Modified > Biotin \\
\hline 48 & CCAGGCGCTTAATCATTGTGAATTACAGGTAG \\
\hline 49 & AGTAATCTTAAATTGGGCTTGAGAGAATACCA \\
\hline 50 & CCAAATCACTTGCCCTGACGAGAACGCCAAAA \\
\hline 51 & TTATTCATAGGGAAGGTAAATATTCATTCAGT \\
\hline 52 & GACTTGAGAGACAAAAGGGCGACAAGTTACCA \\
\hline 53 & AATCACCAAATAGAAAATTCATATATAACGGA \\
\hline 54 & Modified > Biotin \\
\hline 57 & CGATTTTAGAGGACAGATGAACGGCGCGACCT \\
\hline 58 & $\begin{array}{l}\text { TTTCAACTATAGGCTGGCTGACCTTGTATCAT } \\
\text { Modified > CF } 660 \mathrm{C}\end{array}$ \\
\hline 59 & ACGAGTAGTGACAAGAACCGGATATACCAAGC \\
\hline 60 & GAATAAGGACGTAACAAAGCTGCTGACGGAAA \\
\hline 61 & ATTGAGGGTAAAGGTGAATTATCAATCACCGG \\
\hline 62 & AGCGCCAACCATTTGGGAATTAGATTATTAGC \\
\hline 63 & TCACAATCGTAGCACCATTACCATCGTTTTCA \\
\hline 64 & ACGCAAAGGTCACCAATGAAACCAATCAAGTT \\
\hline 65 & ACGAACTAGCGTCCAATACTGCGGAATGCTTT \\
\hline 66 & AAAGATTCAGGGGGTAATAGTAAACCATAAAT \\
\hline 67 & CATTCAACGCGAGAGGCTTTTGCATATTATAG \\
\hline 68 & GGAATTACTCGTTTACCAGACGACAAAAGATT \\
\hline 69 & AAAAGTAATATCTTACCGAAGCCCAACACTAT \\
\hline 70 & GAAGGAAAATAAGAGCAAGAAACAACAGCCAT \\
\hline 71 & ATACCCAAGATAACCCACAAGAATAAACGATT \\
\hline 72 & TTATTACGGTCAGAGGGTAATTGAATAGCAGC \\
\hline 75 & $\begin{array}{l}\text { ACTGGATAACGGAACAACATTATTACCTTATG } \\
\text { Modified > CF660C/ATTO 647N }\end{array}$ \\
\hline 76 & TTTGCCAGATCAGTTGAGATTTAGTGGTTTAA \\
\hline 77 & $\begin{array}{l}\text { CCAAAATATAATGCAGATACATAAACACCAGA } \\
\text { Modified > CF660C/ATTO } 665\end{array}$ \\
\hline 78 & CATAACCCGAGGCATAGTAAGAGCTTTTTAAG \\
\hline 79 & GCAATAGCGCAGATAGCCGAACAATTCAACCG \\
\hline 80 & GCCCAATACCGAGGAAACGCAATAGGTTTACC \\
\hline 81 & ATCAGAGAAAGAACTGGCATGATTTTATTTTG \\
\hline 82 & TGAACAAACAGTATGTTAGCAAACTAAAAGAA \\
\hline
\end{tabular}

\begin{tabular}{|c|c|}
\hline 83 & AACAGTTGATGGCTTAGAGCTTATTTAAATA \\
\hline 84 & CAAAAATCATTGCTCCTTTTGATAAGTTTCAT \\
\hline 85 & TCAGAAGCCTCCAACAGGTCAGGATCTGCGAA \\
\hline 86 & AAGAGGAACGAGCTTCAAAGCGAAGATACATT \\
\hline 87 & CCTAATTTACGCTAACGAGCGTCTATATCGCG \\
\hline 88 & ATTATTTAACCCAGCTACAATTTTCAAGAACG \\
\hline 89 & TTTTGTTTAAGCCTTAAATCAAGAATCGAGAA \\
\hline 90 & СТTI \\
\hline 93 & TTTTTGCGCAGAAAACGAGAATGAATGTTTAG \\
\hline 94 & $\begin{array}{l}\text { TACCTTTAAGGTCTTTACCCTGACAAAGAAGT } \\
\text { Modified > CF } 660 \mathrm{C}\end{array}$ \\
\hline 95 & GAAGCAAAAAAGCGGATTGCATCAGATAAAAA \\
\hline 96 & TTTTAATTGCCCGAAAGACTTCAATTCCAGAG \\
\hline 97 & ATAAATGAAATA \\
\hline 98 & TATTTTGCTCCCAATO \\
\hline 99 & AGGTTTTGAACGTCAAAAATGAAAGCGCTAAT \\
\hline 100 & GAGGCGTTAGAGAATE \\
\hline 101 & TGCAACTAAGCAATAAAGCCTCZ \\
\hline 102 & TCCATATACATACAGGCAAG \\
\hline 103 & Modified > Biotin \\
\hline 104 & TCGCAAATGGGGCGCGAGCTGAAATAATGTGT \\
\hline 105 & ATCGGCTGCGAGCATGTAGAAACCAGCTATAT \\
\hline 106 & Modified > Biotin \\
\hline 107 & CAAGCAAGACGCGCCTGTTTA: \\
\hline 108 & TCATTACCCGACAATAAACAACATATTTAGGC \\
\hline 109 & TATAGAAGCGACAAAAGGTAAAGTAGAGAATA \\
\hline 110 & GCTAAATCCTGTAGCTCAACATGTATTGCTGA \\
\hline 111 & $\begin{array}{l}\text { CAAAATTAAAGTACGGTGTCTGGAAGAGGTCA } \\
\text { Modified > Alexa Fluor } 647\end{array}$ \\
\hline 112 & CAATAAATACAGTTGATTCCCAATTTAGAGAG \\
\hline 113 & $\begin{array}{l}\text { TCAATTCTTTTAGTTTGACCATTACCAGACCG } \\
\text { Modified > Alexa Fluor } 647\end{array}$ \\
\hline 114 & TTTCATTTGGTCAATAACCTGTTTAATCAATA \\
\hline 115 & CTAAT \\
\hline 116 & TAAGTCCTACCAAGTACCGCACTCTTAGTTGC \\
\hline 117 & AATGCAGACCGTTTTTATTTTCATCTTGCGGG \\
\hline 118 & CCAGACGAGCGCCCAATAGCAAGCAAGAACGC \\
\hline 119 & CTGTAATATTGCCTGAGAGTCTGGAAAACTAG \\
\hline 120 & CAACGCAATTTTTGAGAGATCTACTGATAATC \\
\hline 121 & TATATTTTAGCTGATAAATTAATGTTGTATAA \\
\hline 122 & AGGTAAAGAAATCACCATCAATATAATATTTT \\
\hline 123 & GCGTTATAGAAAAAGCCTGTTTAGAAGGCCGG \\
\hline 124 & ACGCTCAAAATAAGAATAAACACCGTGAATTT \\
\hline 125 & CATATTTAGAAATACCGACCGTGTTACCTTTT \\
\hline
\end{tabular}




\begin{tabular}{|c|c|}
\hline & 1 \\
\hline 129 & CAGGTCACTTTTGCGGGAGAAGCAGAATTAG \\
\hline 130 & $\begin{array}{l}\text { GGTAGCTAGGATAAAAATTTTTAGTTAACATC } \\
\text { Modified > Alexa Fluor } 647\end{array}$ \\
\hline 131 & ACCGTTCTAAATGCAATGCCTGAGAGGTGGCA \\
\hline 132 & AGACAGTCATTCAAAAGGGTGAGATATCATAT \\
\hline 133 & AATTACTACAAATTCTTACCAGTAATCCCATC \\
\hline 134 & AGGCGTTACAGTAGGGCTTAATTGACAATAGA \\
\hline 135 & AATGGTTTACAACGCCAACATGTAGTTCAGCT \\
\hline 136 & TTTTAGTTTTTCGAGCCAGTAATAAATTCTGT \\
\hline 137 & CATGTCA \\
\hline 138 & AGAAAAGCAACATTA \\
\hline 139 & GCAAATATCGCGTCTGGCCTTCC \\
\hline 140 & GTTAAAATTTTAACCAATAGGAACCCGGCACC \\
\hline 141 & TTAAGAC \\
\hline 142 & ATCAAAATCGTCGCTATTAAT \\
\hline 143 & TAACCTCCATATGTGAGTGA \\
\hline 144 & AAATTACCT \\
\hline 147 & ACCCGTCGTCATATGTACCCCGGTAAAGGCTA \\
\hline 148 & CTTTCATCCCCAAAAACAGGAAGACCGGAGAG \\
\hline 149 & AAATAATTTTAAATTGTAAACGTTGATATTCA \\
\hline 150 & GCTCATTTTCGCATTAAATTTTTGAGCTTAGA \\
\hline 151 & TAGAATCCCTGAGAAGAGTCAATAGGAATCAT \\
\hline 152 & CTGTAAATCATAGGTCTGAGAGACGATAAATA \\
\hline 153 & TACTAAATTT \\
\hline 154 & TTGATGCAAATCCACAAATATA \\
\hline 155 & TAGATGGGGGGTAACGCCAGGGTTGTGCCAAG \\
\hline 156 & GTTTGAGGGAAAGGGGGATGTGCTAGAGGATC \\
\hline 157 & GAAGATCGGTGCGGGCCTCTTCGCAATCATGG \\
\hline 158 & GCTTCTGGTCAGGCTGCGCAACTGTGTTATCC \\
\hline 159 & CTTTTACACAGATGAATATACAGTAAGCGCCA \\
\hline 160 & CCTGATTGAAAGAAATTGCGTAGACCCGAACG \\
\hline 161 & GCGCAGAGATATCAAAATTATTTGACATTATC \\
\hline 162 & GAGCAAAAACTTCTGAATAATGGAAGAAGGAG \\
\hline 165 & ATTAAGTTCGCATCGTAACCGTGCGAGTAACA \\
\hline 166 & CAGCTGGCGGACGACGACAGTATCGTAGCCAG \\
\hline 167 & GGCGATCGCACTCCAGCCAGCTTTGCCATCAA \\
\hline 168 & TTCGCCATTGCCGGAAACCAGGCAAACAGTAC \\
\hline 169 & TTTAACGTTCGGGAGAAACAATAATTTTCCCT \\
\hline 170 & ACAGAAATCTTTGAATACCAAGTTCCTTGCTT \\
\hline 171 & AACCTACCGCGAATTATTCATTTCCAGTACAT \\
\hline 172 & TGGATTATGAAGATGATGAAACAAAATTTCAT \\
\hline
\end{tabular}

\section{Modified > Biotin}

174 CCCGGGTACTTTCCAGTCGGGAAACGGGCAAC

175 TCATAGCTACTCACATTAATTGCGCCCTGAGA

176 GCTCACAATGTAAAGCCTGGGGTGGGTTTGCC

177 CGACAACTAAGTATTAGACTTTACAGCCGGAA

178 TTATTAATGCCGTCAATAGATAATCAGAGGTG

179 ATtTTGCGTCTTTAGGAGCACTAAGCAACAGT

180 Modified > Biotin

183 GCCAGCTGCCTGCAGGTCGACTCTGCAAGGCG

184 ACTGCCCGCCGAGCTCGAATTCGTTATTACGC

185 GTGAGCTAGTTTCCTGTGTGAAATTTGGGAAG

186 GCATAAAGTTCCACACAACATACGAAACAATT

187 GGATTTAGCGTATTAAATCCTTTGTTTTCAGG

188 AGATTAGATTTAAAAGTTTGAGTACACGTAAA

189 CTAAAATAGAACAAAGAAACCACCAGGGTTAG

190 ATCAACAGTCATCATATTCCTGATTGATTGTT

191 TGGTTTTTAACGTCAAAGGGCGAAGAACCATC

192 AgCTGATTACAAGAGTCCACTATTGAGGTGCC

193 GAGTTGCACGAGATAGGGTTGAGTAAGGGAGC

194 CCAGCAGGGGCAAAATCCCTTATAAAGCCGGC

195 ACGAACCAAAACA TCGCCATTAAATGGTGGTT

196 AgGCGGTCATTAGTCTTTAATGCGCAATATTA

197 GCCACGCTATACGTGGCACAGACAACGCTCAT

198 CTAAAGCAAGATAGAACCCTTCTGAATCGTCT

201 TGGACTCCCTTTTCACCAGTGAGACCTGTCGT

202 AGTTtGGAGCCCTTCACCGCCTGGTTGCGCTC

203 GAATAGCCGCAAGCGGTCCACGCTCCTAATGA

204 CCGAAATCCGAAAATCCTGTTTGAAATACCGA

205 TAGCCCTACCAGCAGAAGATAAAAACATTTGA

206 GAATGGCTAGTATTAACACCGCCTCAACTAAT

207 GCGTAAGAGAGAGCCAGCAGCAAAAAGGTTAT

208 GCCAACAGTCACCTTGCTGAACCTGTTGGCAA

210 ACCCAAATCAAGTTTTTTGGGGTCAAAGAACG

211 GTAAAGCACTAAATCGGAACCCTAGTTGTTCC

212 CCCCGATTTAGAGCTTGACGGGGAAATCAAAA

213 GAACGTGGCGAGAAAGGAAGGGAACAAACTAT

214 CGGCCTTGCTGGTAATATCCAGAACGAACTGA

215 CCGCCAGCCATTGCAACAGGAAAAATATTTTT

216 GGAAATACCTACATTTTGACGCTCACCTGAAA

217 GAAATGGATTATTTACATTGGCAGACATTCTG 


\section{ACKNOWLEDGEMENTS}

This dissertation would not have been possible without the contributions from numerous highly skilled and talented people. I would like to acknowledge their investment of time and critical thinking and express my deep thankfulness for their support.

Foremost, my sincere gratitude to Stefan Hell for affording me the opportunity to perform this exciting work, for his continuous interest and support of my research projects, for his outstanding new ideas as well as for the inspiring discussions that always opened new perspectives.

I thank Sarah Köster and Claus Ropers for examining my thesis and for their great support and time while being part of my thesis advisory committee. I am indebted to Gopalakrishnan Balasubramanian, Stefan Jakobs and Silvio Rizzoli for being part of the examination board for my thesis.

Our collaborators Jonas Ries, Jan Ellenberg and Philipp Hoess from Heidelberg proposed and provided the U-2 OS Nup96-SNAP cell line and carefully prepared the samples, I am grateful for their contribution. I thank Dirk Kamin and Ina Maria Herfort for handling the hippocampal neuron cultures and helping me with the sample preparation for the measurement of the PSD-95 distribution. The mouse model was kindly provided by Seth Grant from Edinburgh. I am especially grateful to Stefan Jakobs and Till Stephan for providing brilliant ideas, cell samples and continuous support that facilitated the establishment of MINFLUX imaging in mitochondria. I thank Haisen Ta for sharing his experience, especially concerning DNA origami assembly and buffer optimization. I appreciate the help of Mark Bates in form of many interesting discussions about numerous experimental details. I thank Rebecca Büchner for her excellent and independent work in the lab that supported the progress of this thesis.

I sincerely appreciate the supportive assistance especially by Marco Roose, Tanja Gilat, Ellen Rothermel, Robert Braunschweig and Jaydev Jethwa, but also by the 
secretaries, the facility for synthetic chemistry, the media service and the institute's workshops for optics, electronics and precision mechanics. Klaus Gwosch, Till Stephan, Malte Vassholz, Jan Keller-Findeisen, Jaydev Jethwa and Francisco Balzarotti helped with critical reading and fruitful discussions to make this thesis more accessible to the reader.

I would like to thank all members of the Department of NanoBiophotonics, especially my former and current office mates for generating an inspiring and friendly work atmosphere.

Last, but by no means least, I would like to thank Francisco Balzarotti and Klaus Gwosch who provided generous support throughout my thesis by answering and discussing all kinds of work- or non-work-related questions, with their ideas and honesty, their knowledge, experience and project contributions as well as by welcoming me and guiding me in the highly motivated MINFLUX team. I cannot thank them enough for this exciting and motivating work experience! 